\title{
A fixed-target programme at the LHC: Physics case and projected performances for heavy-ion, hadron, spin and astroparticle studies
}

\section{Hadjidakis ${ }^{1, a}$, D. Kikoła ${ }^{2, a}$, J.P. Lansberg ${ }^{1, *, a}$, L. Massacrier ${ }^{1, a}$, M.G. Echevarriaa $3,4, b$, A. Kusina ${ }^{5, b}$, I. Schienbein ${ }^{6, b}$, J. Seixas ${ }^{7,8,9, b}$, H.S. Shao ${ }^{10, b}$, A. Signori ${ }^{11,3,12, b}$, B. Trzeciak ${ }^{13,14, b}$, S.J. Brodsky ${ }^{15}$, G. Cavoto ${ }^{16}$, C. Da Silva ${ }^{17}$, F. Donato ${ }^{18}$, E.G. Ferreiro ${ }^{19,20}$, I. Hřivnáčová ${ }^{1}$, A. Klein ${ }^{17}$, A. Kurepin ${ }^{21}$, C. Lorcé $^{22}$, F. Lyonnet ${ }^{23}$, Y. Makdisi ${ }^{24}$, S. Porteboeuf Houssais ${ }^{25}$, C. Quintans ${ }^{8}$, A. Rakotozafindrabe ${ }^{26}$, P. Robbe ${ }^{1}$, W. Scandale ${ }^{27}$, N. Topilskaya ${ }^{21}$, A. Uras ${ }^{28}$, J. Wagner ${ }^{29}$, N. Yamanaka ${ }^{1,32,30,31}$, Z. Yang ${ }^{33}$, A. Zelenski ${ }^{24}$}

${ }^{1}$ Université Paris-Saclay, CNRS, IJCLab, 91405 Orsay, France

${ }^{2}$ Faculty of Physics, Warsaw University of Technology, ul. Koszykowa 75, 00-662 Warsaw, Poland

${ }^{3}$ Istituto Nazionale di Fisica Nucleare, Sezione di Pavia, via Bassi 6, 27100 Pavia, Italy

${ }^{4}$ Dpto. de Física y Matemáticas, Universidad de Alcalá, 28805 Alcalá de Henares (Madrid), Spain

${ }^{5}$ Institute of Nuclear Physics Polish Academy of Sciences, PL-31342, Krakow, Poland

${ }^{6}$ Laboratoire de Physique Subatomique et de Cosmologie, Université Grenoble Alpes, CNRS/IN2P3, 53 Avenue des Martyrs, F-38026 Grenoble, France

${ }^{7}$ Dep. Fisica, Instituto Superior Tecnico, Av. Rovisco Pais 1, 1049-001 Lisboa, Portugal

${ }^{8}$ LIP, Av. Prof. Gama Pinto, 2, 1649-003 Lisboa, Portugal

${ }^{9}$ Centro de Física e Engenharia de Materiais Avançados, Av. Rovisco Pais 1, 1049-001 Lisboa, Portugal

${ }^{10}$ LPTHE, UMR 7589, Sorbonne Université et CNRS, 4 place Jussieu, 75252 Paris Cedex 05, France

${ }^{11}$ Theory Center, Thomas Jefferson National Accelerator Facility, 12000 Jefferson Avenue, Newport News, VA 23606, USA

${ }^{12}$ Dipartimento di Fisica, Università di Pavia, via Bassi 6, I-27100 Pavia, Italy

${ }^{13}$ Institute for Subatomic Physics, Utrecht University, Utrecht, The Netherlands

${ }^{14}$ Faculty of Nuclear Sciences and Physical Engineering, Czech Technical University in Prague, Prague, Czech Republic

${ }^{15}$ SLAC National Accelerator Laboratory, Stanford University, Menlo Park, CA 94025, USA

16 "Sapienza“ Università di Roma, Dipartimento di Fisica E INFN, Sez. di Roma, P.le A. Moro 2, 00185 Roma, Italy

${ }^{17}$ P-25, Los Alamos National Laboratory, Los Alamos, NM 87545, USA

${ }^{18}$ Turin University, Department of Physics, and INFN, Sezione of Turin, Turin, Italy

${ }^{19}$ Dept. de Física de Partículas E IGFAE, Universidade de Santiago de Compostela, 15782 Santiago de Compostela, Spain

${ }^{20}$ Laboratoire Leprince-Ringuet, Ecole polytechnique, CNRS/IN2P3, Palaiseau, France

${ }^{21}$ Institute for Nuclear Research, Russian Academy of Sciences, 117312 Moscow, Russia

${ }^{22}$ CPHT, CNRS, Ecole Polytechnique, Institut Polytechnique de Paris, Route de Saclay, 91128 Palaiseau, France

${ }^{23}$ Southern Methodist University, Dallas, TX 75275, USA

${ }^{24}$ Brookhaven National Laboratory, Collider Accelerator Department, USA

${ }^{25}$ Université Clermont Auvergne, CNRS/IN2P3, LPC, F-63000 Clermont-Ferrand, France

${ }^{26}$ IRFU/DPhN, CEA Saclay, 91191 Gif-sur-Yvette Cedex, France

${ }^{27}$ CERN, European Organization for Nuclear Research, 1211 Geneva 23, Switzerland

${ }^{28}$ IPNL, Université Claude Bernard Lyon-I and CNRS-IN2P3, Villeurbanne, France

${ }^{29}$ National Centre for Nuclear Research (NCBJ), Pasteura 7, 02-093 Warsaw, Poland

${ }^{30}$ Amherst Center for Fundamental Interactions, Department of Physics, University of Massachusetts Amherst, MA 01003, USA

${ }^{31}$ Nishina Center for Accelerator-Based Science, RIKEN, Wako 351-0198, Japan

\footnotetext{
* Corresponding author.

E-mail address: lansberg@in2p3.fr (J.P. Lansberg).

a Editor.

b Section editor.
} 
${ }^{32}$ Department of Physics, Kennesaw State University, Kennesaw, GA 30144, USA

${ }^{33}$ Center for High Energy Physics, Department of Engineering Physics, Tsinghua University, Beijing, China

\section{A R T I C L E I N F O}

Article history:

Received 6 October 2020

Received in revised form 26 January 2021

Accepted 26 January 2021

Available online 14 February 2021

Editor: M. Ramsey-Musolf

\begin{abstract}
A B S T R A C T
We review the context, the motivations and the expected performances of a comprehensive and ambitious fixed-target programme using the multi-TeV proton and ion LHC beams. We also provide a detailed account of the different possible technical implementations ranging from an internal wire target to a full dedicated beam line extracted with a bent crystal. The possibilities offered by the use of the ALICE and LHCb detectors in the fixed-target mode are also reviewed.
\end{abstract}

(C) 2021 Elsevier B.V. All rights reserved.

\section{Contents}

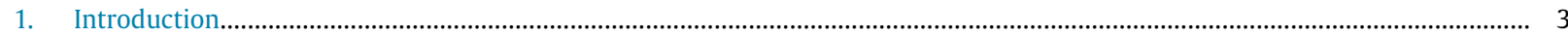

2. Motivations …

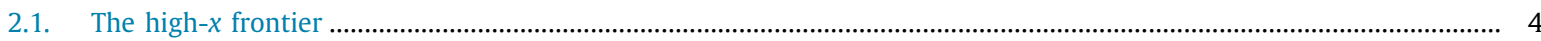

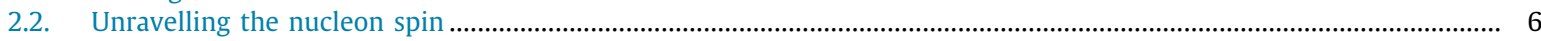

2.3. The nuclear matter in new rapidity and energy domains.................................................................................... 8

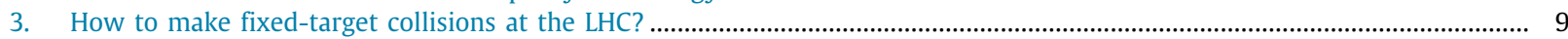

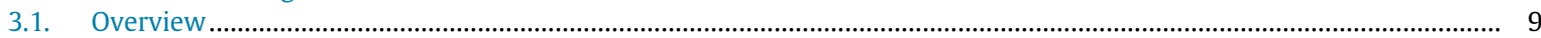

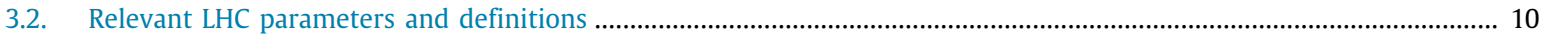

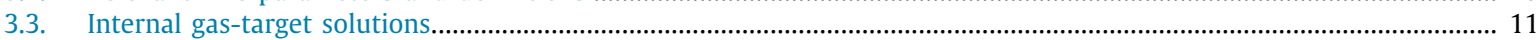

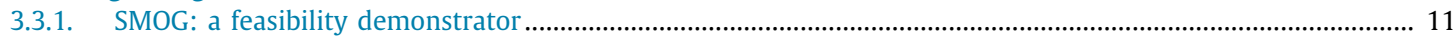

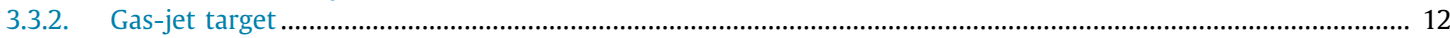

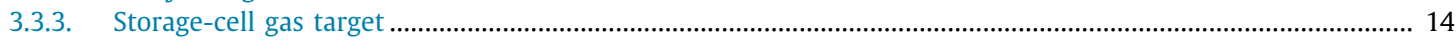

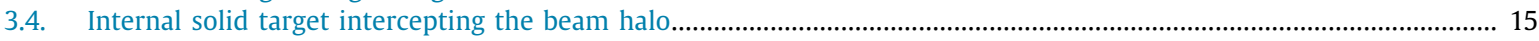

3.5. External/internal target solution with a slow beam extraction using a bent crystal ..................................................... 16

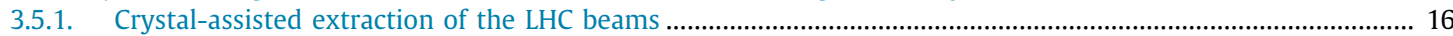

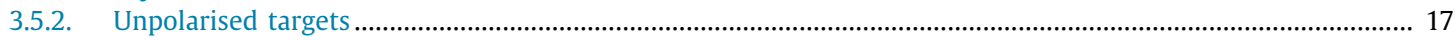

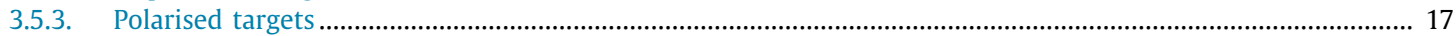

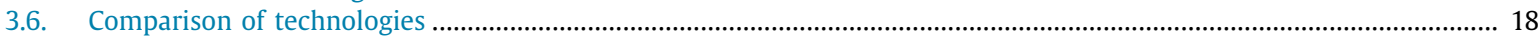

3.6.1. Qualitative comparison of the various technological solutions................................................................. 18

3.6.2. Comparison of the luminosities achieved for AFTER@LHC with the various technological solutions............... 19

3.6.3. Comparison of the polarised-target performances for STSA measurements .................................................. 19

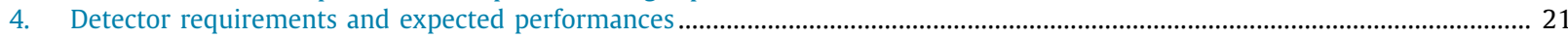

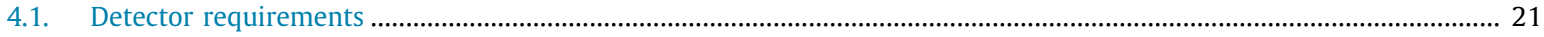

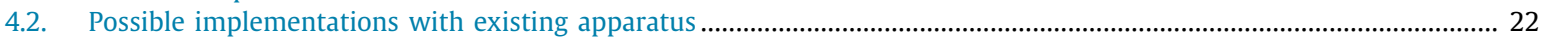

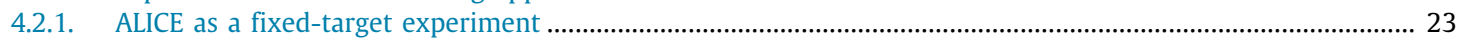

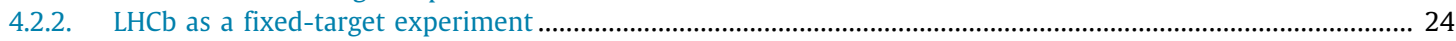

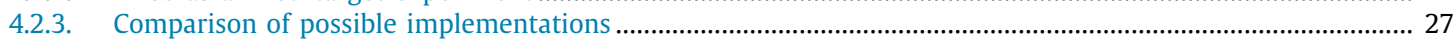

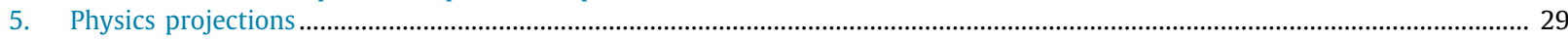

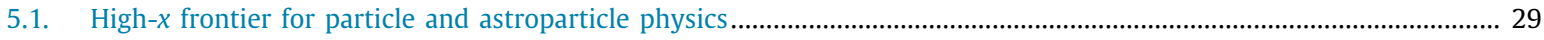

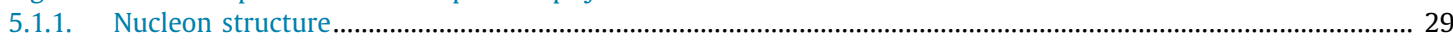

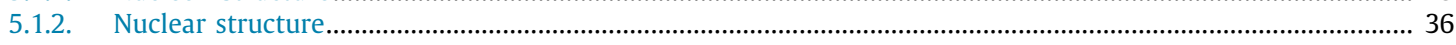

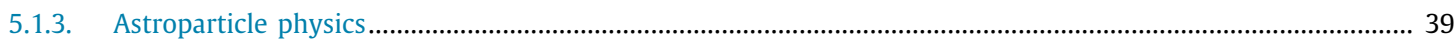

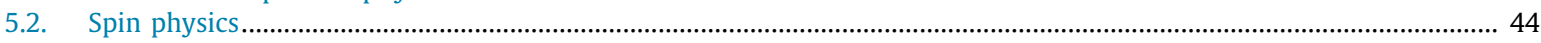

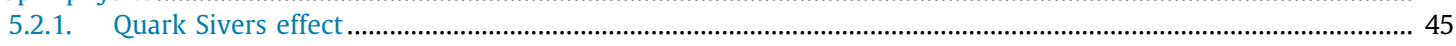

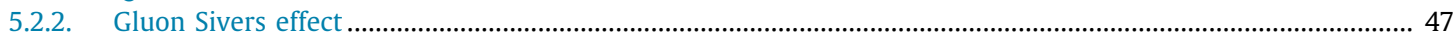

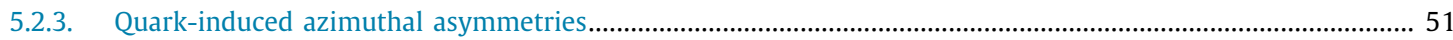

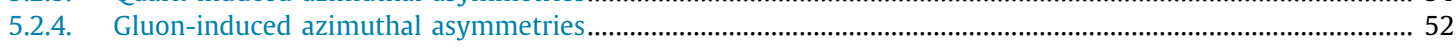

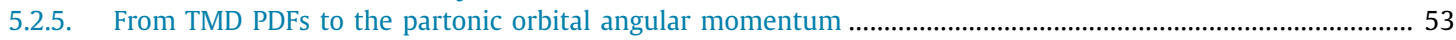

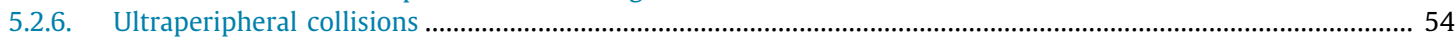

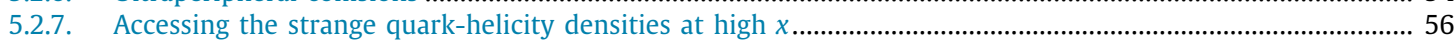

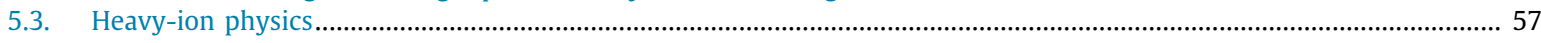

5.3.1. Precise quarkonium studies in new rapidity and energy domains ............................................................. 58

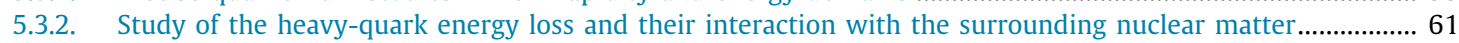

5.3.3. Soft probes at large rapidities - a precise tool to study the bulk properties of the nuclear matter..................62 62

5.3.4. Search for a collective dynamics of partons in small systems at low energies .............................................. 64

5.3.5. Test of the factorisation of the initial-state nuclear effects in AA collisions with Drell-Yan lepton-pair

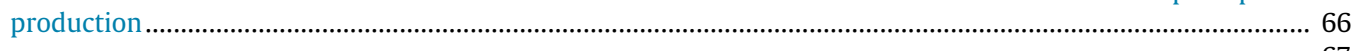

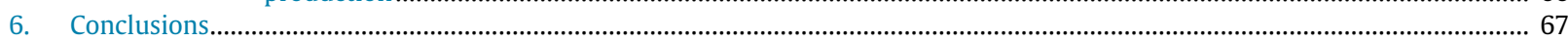




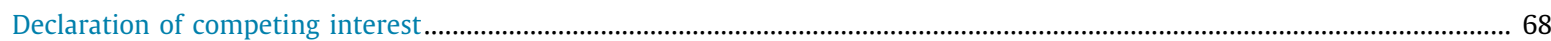

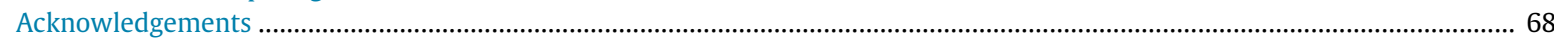

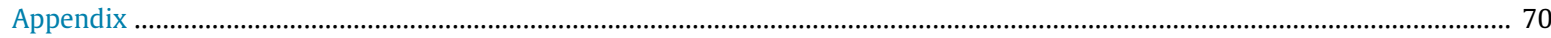

A.1. Schematic view of the H-jet system used at the BNL-RHIC collider ............................................................................ 70

A.2. Possible setup of the beam-splitted option upstream of LHCb .................................................................................... 70

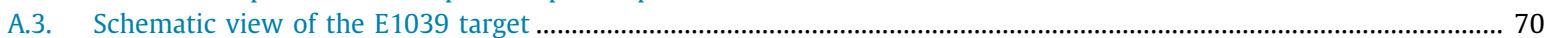

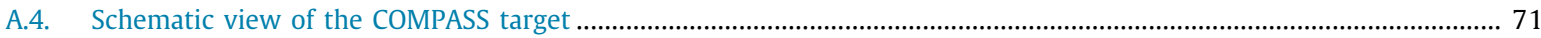

A.5. Schematic view of the ALICE detectors............................................................................................................... 71

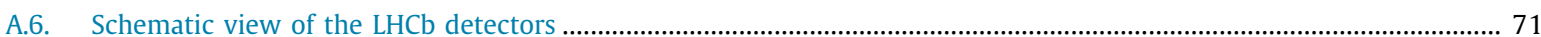

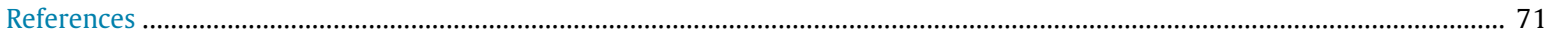

\section{Introduction}

The objective of this review is to highlight the physics opportunities of using the most energetic proton and ion beams ever in the fixed-target mode and to review the feasibility of a rich physics programme for heavy-ion, hadron, spin and astroparticle physics with existing (LHCb or ALICE) or new set-ups allowing one to perform such studies parasitically for the LHC collider programme.

Let us first recall that the fixed-target mode offers several unique assets [1] compared to the collider mode which are particularly relevant with the LHC beams in the context of high-energy physics :

- A high luminosity thanks to the high density of the target at no cost for the LHC collider-mode experiments. Both an internal gas target or a bent-crystal-extracted beam from the beam halo allow for yearly luminosities well above those of similar machines, in particular RHIC, in the ballpark of the LHC and Tevatron collider luminosities;

- The accessibility with standard detectors, thanks to the boost between the colliding-nucleon centre-of-mass system (c.m.s.) and the laboratory system, to the far backward c.m.s. region which remains completely uncharted with hard reactions until now. A pseudo-rapidity acceptance of $1 \leq \eta_{\text {(lab.) }} \leq 5$, combined with high luminosities, essentially allows one to measure any probe down to the very end of the backward phase space;

- An extended number of species for the target, including deuteron and ${ }^{3} \mathrm{He}$ allowing for unique neutron studies, with the possibility to change them in a reduced amount of time for short runs;

- The c.m.s. energy per nucleon-nucleon collision $\left(\sqrt{S_{N N}}\right)$ is identical for all $7 \mathrm{TeV}$ proton and $2.76 \mathrm{TeV}$ lead induced collisions, namely $115 \mathrm{GeV}$ for $p p, p d, p A$ systems and $72 \mathrm{GeV}$ for $\mathrm{Pb} p, \mathrm{~Pb} d, \mathrm{~Pb} A$ systems. ${ }^{1}$ This allows for nuclearmodification-factor measurements with drastically reduced systematic uncertainties in an energy domain between the SPS and RHIC experiments in an unexplored rapidity domain;

- The target polarisation - whereas the LHC beams are unpolarised. This offers uncountable opportunities for single spin asymmetry (SSA) measurements - at large momentum fractions - which have been the object of a growing attention in the recent years at RHIC, at CERN and at Fermilab.

Owing to these advantages, we have identified three main topics for a strong physics case motivating a complete fixed-target programme at the LHC (referred to AFTER@LHC in what follows) with one or more detectors. These cover studies of

- the high momentum fraction $(x)$ frontiers in nucleons and nuclei with a specific emphasis on the gluon and heavy-quarks and the implication for astroparticle physics;

- the spin content of the nucleons with a focus on azimuthal asymmetries generated by the spin of the partons and Single Transverse-Spin Asymmetries (STSAs) generated by the correlation between the nucleon spin and the momentum of partons;

- the hot medium created in ultra-relativistic heavy-ion collisions with novel quarkonium and heavy-quark observables in a new energy domain and with identified light hadrons down to the target-rapidity region.

The structure of this review is as follows. In Section 2, we quickly review the context in which such a AFTER@LHC programme would take place and highlight the motivations for the three main aforementioned research axes. In Section 3, we provide a state-of-the-art overview of the different available technologies to initiate collisions of the LHC beams with fixed targets. In Section 4, we elaborate on the detector aspects both for an ideal detector and for existing detectors, i.e. those of the LHCb and ALICE collaborations. ${ }^{2}$ In Section 5, we extensively review the projected performances for flagship studies and the studies proposed within the community for each of the 3 main topics. Section 6 gathers our conclusions.

\footnotetext{
$1 \sqrt{s_{N N}}$ for lighter ion beams remains on the order of $70 \mathrm{GeV}$.

2 In what follows, AFTER@LHC will refer to a generic experimental fixed-target set-up using the LHC beams, AFTER@LHCb, AFTER@ALICE, AFTER@ALICE $_{\mu}$ and AFTER@ALICE ${ }_{\text {CB }}$ to specific implementations using the LHCb or ALICE detectors.
} 


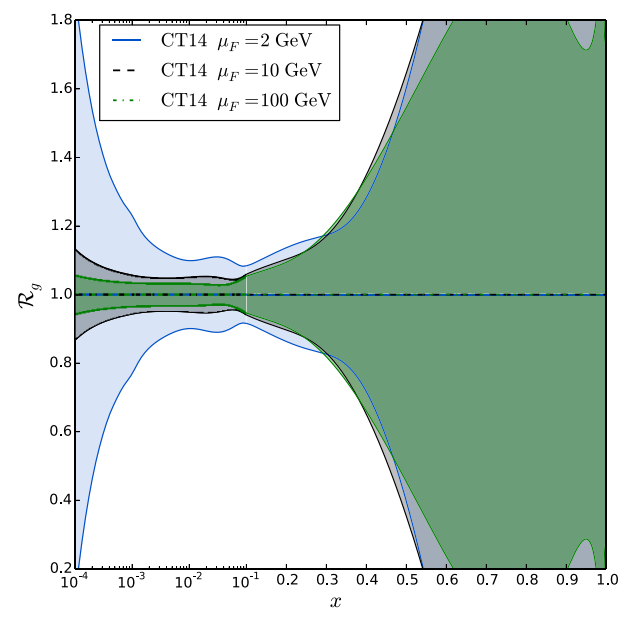

(a)

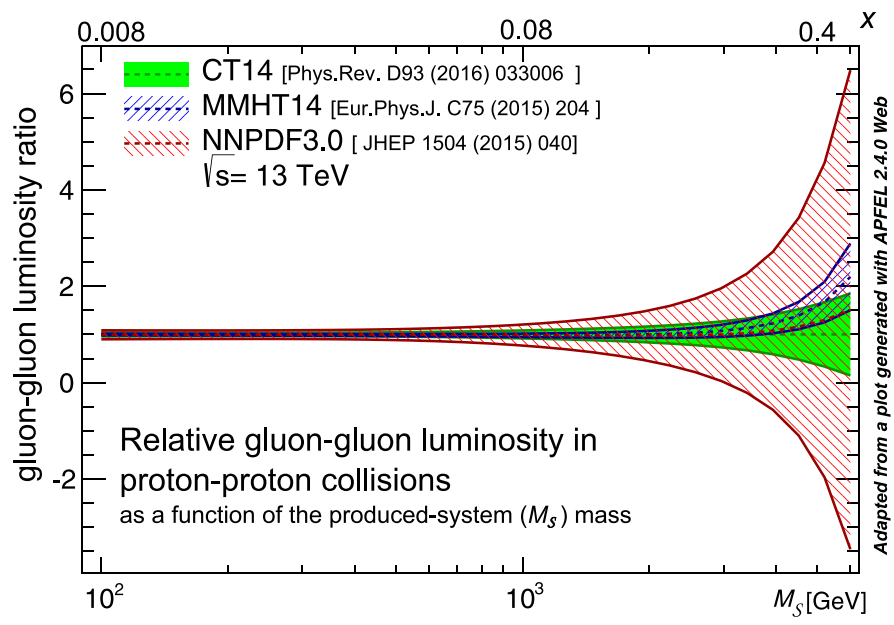

(b)

Fig. 1. (a) CT14nlo gluon PDF relative uncertainties [5] in a proton as a function of the gluon momentum fraction $x$ at three values of the factorisation scale, $\mu_{F}$, (b) Gluon-gluon-luminosity uncertainty computed for three sets of proton PDFs as a function of the invariant mass $\left(M_{\mathcal{S}}\right)$ of a to-be produced system at $\sqrt{s}=13 \mathrm{TeV}$. For $y \sim 0, x \simeq M_{\mathcal{S}} / \sqrt{s}$ at the LHC (indicated on the upper $x$ axis). The kinematics of the AFTER@LHC programme is mainly that of high $x$ where the uncertainties blow up. Plot done thanks to the APFEL programme [6].

\section{Motivations}

\subsection{The high-x frontier}

Whereas the need for precise measurements of the partonic structure of nucleons and nuclei at small momentum fractions $x$ is usually highlighted as a strong motivation for new large-scale experimental facilities, such as the ElectronIon Collider [2] (EIC) or Large Hadron-electron Collider [3] (LHeC) projects, the structure of nucleons and nuclei at high $x$ is as poorly known at both low and high scales (see Fig. 1(a)). Let us mention the long-standing puzzles such as the origin of the nuclear $\mathrm{EMC}^{3}$ effect in nuclei or a possible non-perturbative source of charm or beauty quarks in the proton which would carry a relevant fraction of its momentum. With an extensive coverage of the backward region corresponding to high $x$ in the target, AFTER@LHC is probably the best programme for such physics with hadron beams.

Studying the so-called high- $x$ physics also provides us with novel decisive means to advance our experimental knowledge of the still poorly understood confinement properties of the strong interaction, which is one of the last open questions about the Standard Model. Indeed, studying high- $x$ fluctuations of a nucleon, where a single gluon carries the majority of the confined-system momentum, certainly tests QCD in a new limit never explored before. On the quark side, an improved experimental determination of the $d / u$ PDF ratio for $x \rightarrow 1$ is also crucial to tell which picture is valid between an SU(6) symmetric one where $d / u \rightarrow 1 / 2$, the dominance of a quark-scalar diquark where $d / u \rightarrow 0$, quarkhadron duality where $d / u \rightarrow 0.42$ or a simple perturbative QCD one where $d / u \rightarrow 1 / 5$. Beside such fundamental issues touching upon our understanding of confinement, charting the high- $x$ structure of nucleons and nuclei has very practical implications, for instance to improve our knowledge of parton luminosities at existing and future hadron colliders (LHC, RHIC, Tevatron, FCC, ... ) (see Fig. 1(b)) but also of Ultra-High-Energy-Cosmic Rays (UHECR), in particular the neutrino, in the PeV range.

Beyond unbound nucleons, our understanding of the gluon and quark content of the nuclei is also very limited at high $x$. Since the first observation via DIS measurements of a nuclear suppression of the quark momentum distribution - the aforementioned EMC effect -, DIS data got more precise and confirmed the suppression. Yet, we still do not understand its physical origin. Recently, it was argued that the $x>1$ scaling plateaux of some nucleus structure functions, attributed to short-range nucleon-nucleon correlations related to high local densities in nuclei, could be related to the EMC effect [11]. In this context, the complete lack of data constraining the gluon density in this region (see Fig. 2 ) is probably very detrimental. Only indirect constraints from the scaling violation of the quark distribution exist, which obviously do not give additional experimental information. We are also lacking precise nuclear Drell-Yan (DY) data which provide a unique window on the sea quarks. A precise measurement of the gluon EMC and of its nuclear number $(A)$ dependence, combined with precise DY data at high $x$, would provide decisive insights into the origin of the EMC effects which goes along with understanding how quark and gluons behave in the nuclear medium.

\footnotetext{
3 Named after its observation in 1983 by the European Muon Collaboration [4].
} 


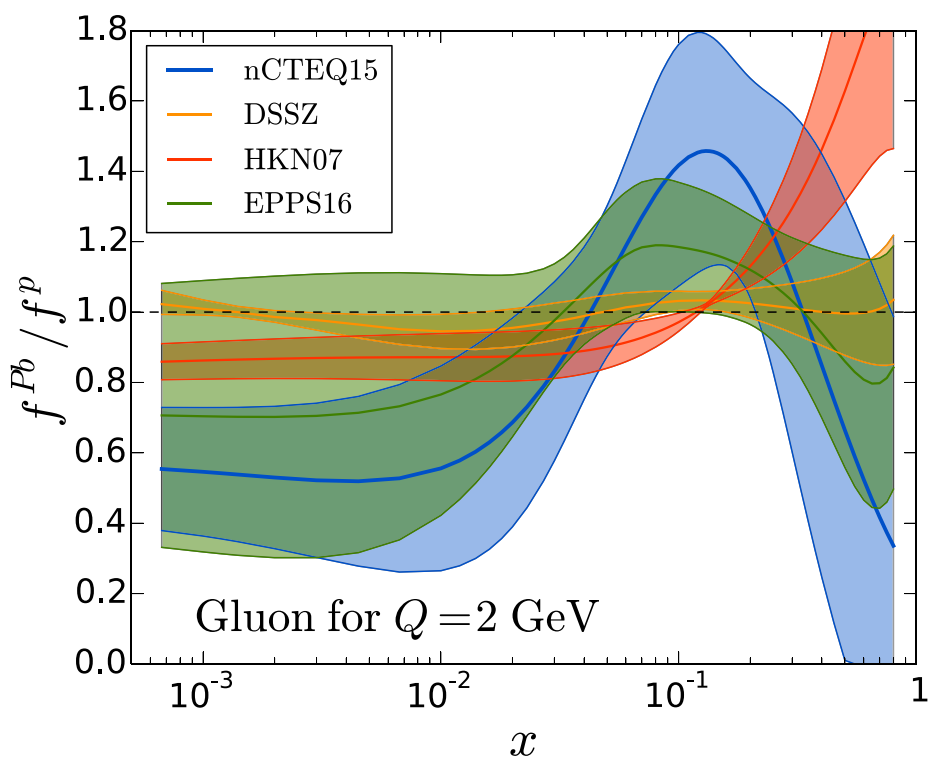

Fig. 2. Compilation of the gluon nuclear PDF relative uncertainties [7-10] in a lead nucleus at a factorisation scale (here denoted Q) of 2 GeV.

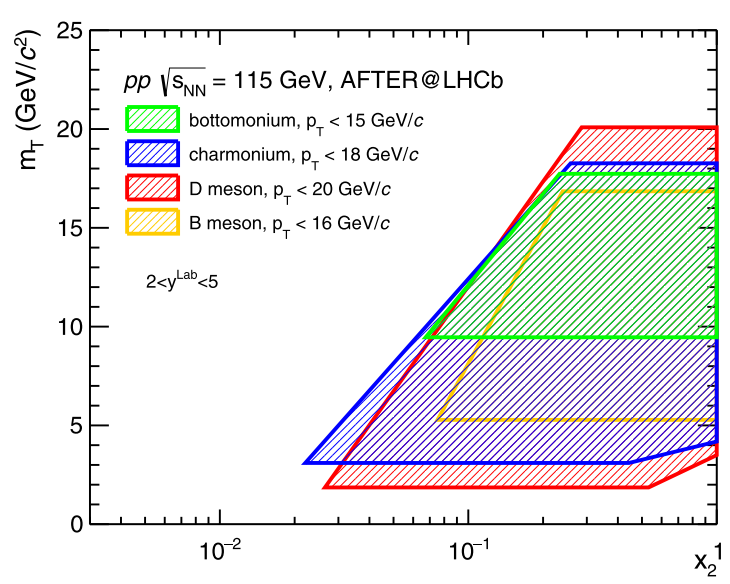

(a) LHCb-like

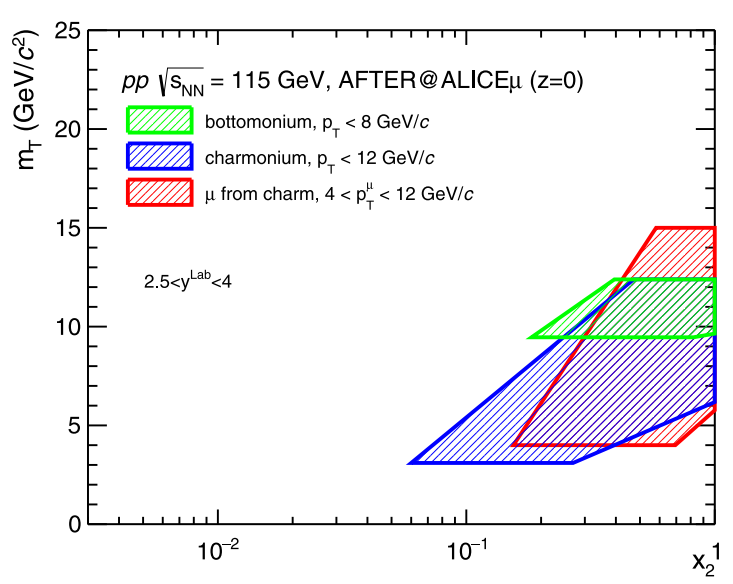

(b) ALICE-like

Fig. 3. Typical kinematical reach with heavy-hadron production in $x_{2}$ (our proxy to the momentum fraction of the parton in the target) and the scale (chosen to be $m_{T}=\sqrt{M_{\text {hadron }}^{2}+P_{T, \text { hadron }}^{2}}$ ) of the fixed-target mode with a detector acceptance like (a) LHCb and (b) ALICE.

At the interface between the proton and nuclear cases, the deuteron and ${ }^{3} \mathrm{He}$ have a particular place. On the one hand, they can provide us with quasi-free neutron targets allowing us to test assumptions such as $u^{p}=d^{n}, \bar{u}^{p}=\bar{d}^{n}$ based on the isospin symmetry, or whether gluons behave differently in protons than in neutrons. On the other hand, it is an appealing playground to test our understanding of the dynamics of simple nucleon-bound systems and hidden colour configurations of six quarks (see e.g. [12]).

For all these reasons, the unique opportunities offered by AFTER@LHC to probe with high precision and with reliable as well as novel perturbative probes the high- $x$ domain are very interesting. Fig. 3 schematically illustrates, with a small set of selected (gluon-sensitive) probes, how the fixed-target mode with multi-TeV beams allows one to methodically probe the high- $x$ region in the target, namely at high $x_{2}$. This obviously applies equally for quark, heavy-quark, gluon and anti-quark sensitive probes in protons, deuterons and light or heavy nuclei. In addition, when heavy ions are used as beam particles, one can probe their contents down to $x$ value as small as 0.005 . The possibility to study the large- $x$-charm content in the (nucleon and nucleus) target will also allow one to severely reduce the uncertainty of the prompt neutrino fluxes.

Finally, let us highlight the opportunities connected to anti-proton $(\bar{p})$ measurements in new kinematical ranges and for new systems in order to further constrain the modelling of the conventional $\bar{p}$ astrophysical sources. One of the possible 


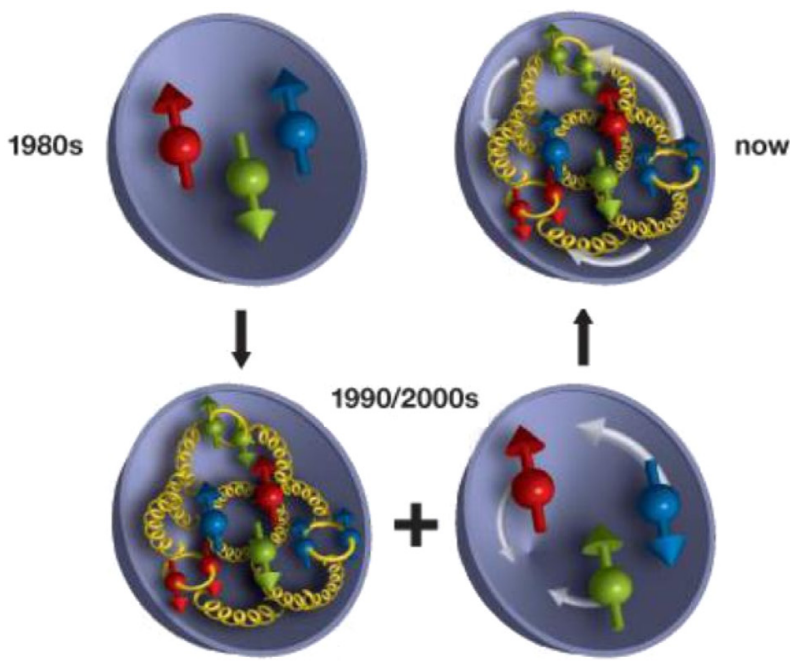

(a)

\section{= Gluon Spin Gluon angular momentum $=$ Quark Spin $=$ Quark Angular Momentum}

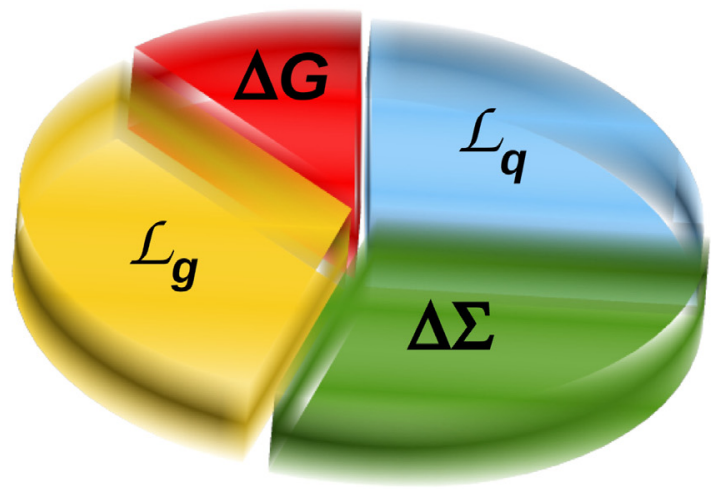

(b)

Fig. 4. (a) Evolution of our understanding of the spin content of the nucleon [adapted from [18]]. (b) Decomposition of the nucleon spin relevant for high-energy processes.

very original measurements is that of $\bar{p}$ nearly at rest from fixed $\mathrm{He}, \mathrm{C}, \mathrm{N}$ or $\mathrm{O}$ targets relying on the Particle IDentification (PID) capabilities of the ALICE central barrel. This would constrain highly-energetic $\bar{p}$ from $(\mathrm{He}, \mathrm{C}, \mathrm{N}, \mathrm{O})+p \rightarrow \bar{p}+X$.

\subsection{Unravelling the nucleon spin}

Despite decades of efforts, the internal structure of the nucleons, in particular their constituent distribution and dynamics, is still largely unknown. One of the most significant issues is our limited understanding of the spin structure of the nucleon, specifically how its elementary constituents (quarks and gluons) bind into a spin- $\frac{1}{2}$ object. Essentially, there are two types of contributions to the nucleon spin from quarks and gluons: their spin and their Orbital Angular Momentum (OAM). For a longitudinally polarised nucleon, i.e. with helicity $+\frac{1}{2}$, one has

$$
\frac{1}{2}=\frac{1}{2} \Delta \Sigma+\Delta G+\mathcal{L}_{g}+\mathcal{L}_{q},
$$

where $\frac{1}{2} \Delta \Sigma$ denotes the combined spin contribution of quarks and anti-quarks, $\Delta G$ the gluon spin, and $\mathcal{L}_{q, g}$ the quark and gluon OAM contributions (see e.g. [13-17]). Eq. (1) is in principle valid at any energy scale at which the nucleon is probed and this calls for the study of the evolution of the individual contributions at different scales. These questions naturally generalise to any spin- $J$ hadron.

We have come a long way since the so-called spin crisis (Fig. 4(a)), faced in the eighties when the measurements performed by the European Muon Collaboration (EMC) revealed that only a small fraction of the nucleon spin was generated by the spin of quarks [19]. The crisis has evolved into a puzzle, which consists now in determining how large the different quark and gluon contributions to the nucleon spin are, in disentangling them and in explaining them from first principles in QCD.

Recent experimental data have shown that the quark and anti-quark spins account for only about $30 \%$ of proton total longitudinal spin [20-23], and the gluon contribution could be as large as $40 \%{ }^{4}$ It is thus expected that a significant part of the proton spin arises from the transverse dynamics of quarks and gluons (i.e. via $\mathcal{L}_{q}$ and $\mathcal{L}_{\mathrm{g}}$ ), which has however not yet been measured. This emphasises how crucial our understanding of the transverse motion of quarks and gluons inside the proton is, in order to validate our current picture of the nucleon structure.

In order to measure the parton OAM, one should in principle access observables which are sensitive to the parton position and momentum. This is the realm of the Generalised Parton Distributions (GPDs), relevant for exclusive processes. Yet, one can also indirectly access information on the orbital motion of the partons bound inside hadrons via Single Spin Asymmetries (SSAs) in different hard-scattering processes, in particular with a transversely polarised hadron $($ see $[26,27]$ for reviews). In these Single Transverse-Spin Asymmetries (STSAs), one can access left-right asymmetries in the parton distributions with respect to the plane formed by the proton momentum and spin directions. These asymmetries are naturally connected to the transverse motion of the partons inside the polarised nucleons.

\footnotetext{
4 The latter has only been measured in the region $x>0.05[24,25]$. Additional studies at smaller $x$ are thus required.
} 
Historically large STSAs (also denoted $A_{N}$ or $A_{U T}$ ) have been observed in single forward $\pi$ [28-30] and $K$ production [29] in high-energy $p^{\uparrow} p$ collisions at Fermilab and Brookhaven National Laboratory (BNL), towards the valence region. They have also been observed in Semi-Inclusive DIS (SIDIS) by the HERMES [31] and COMPASS [32] collaborations. Studies to look for STSAs in $J / \psi$ production in $p^{\uparrow} p$ collisions at RHIC [33] and $J / \psi$ leptoproduction on proton target by the COMPASS experiment [34] have also been carried out. Intense theoretical works have resulted in a widely accepted picture according to which these (large) asymmetries, which were expected to vanish at high energies, are due to re-scatterings of the quarks and gluons with the remnants of the hadron undergoing the interaction [35-37]. It is also accepted that they vanish for partons which do not carry any transverse momentum.

As for now, these STSAs can be treated via two dual approaches [38]. The first is an extension of the collinear parton model of Feynman and Bjorken with the introduction of three-parton (Efremov-Teryaev-Qiu-Sterman) correlations [39-41], namely the Collinear Twist-3 (CT3) formalism. ${ }^{5}$ The second, called the Transverse-Momentum Dependent (TMD) factorisation (see e.g. [44-55]), relies on a complete three-dimensional mapping of the parton momentum and encodes all the possible spin-spin and spin-orbit correlations between the hadron and its constituents. In particular, the effect of the aforementioned re-scatterings, responsible for the STSAs, is encoded into the gauge links in the definition of the TMD PDFs. A particular case is the so-called Sivers function [56,57]. Within the CT3 formalism, the re-scattering effects are explicitly considered in the hard-scattering coefficients.

Both formalisms have their proper range of applicability. While the CT3 approach holds when one single hard scale is present in the process (such as the transverse momentum $p_{T}$ of a pion produced in hadronic collisions with $\Lambda_{\mathrm{QCD}}<p_{T}$ ), the TMD formalism applies when there are two separate scales (such as the transverse momentum $q_{T}$ and the invariant mass $M$ of the lepton pair in DY production, with $\left.\Lambda_{\mathrm{QCD}} \leq q_{T}<M\right)$. Thus the CT3 approach is better suited to describe $A_{N}$ for inclusive hadron production [58] but can also be applied to DY pair [59-61] or isolated photon [62] production. With regards to the AFTER@LHC physics case, this mainly concerns heavy-flavour and quarkonium production, whose STSAs are extremely poorly known, if not unknown at all. TMD factorisation is usually used for processes where the transverse momentum of the initial partons is accessible owing to the absence of hard final-state radiations. In the case of AFTER@LHC, this includes pseudo-scalar quarkonium, quarkonium-pair and other associated production of colourless particles, and of course the DY process. With its high luminosity, a highly polarised target and an access towards the large momentum fraction, $x^{\uparrow}$, in the target, AFTER@LHC is probably the best set-up to carry out an inclusive set of $A_{N}$ measurements both to improve existing analyses and to perform studies which would simply be impossible otherwise. Let us recall that nearly nothing is known from the experimental side about the gluon Sivers function (see e.g. the review [63] and recent measurements [33,64-66]). The polarisation of not only hydrogen but also deuterium and helium targets allows for an even more ambitious spin programme relying on the neutron and spin-1 bound states (see e.g. [52,54] and references therein).

As explained, the TMD approach allows us to investigate the structure of hadrons in a three-dimensional momentum space (see [55] and references therein) in a rigorous and systematic way. It is important to note that it is not restricted to the study of $A_{N}$. It can help us probe in a direct manner the transverse dynamics of the partons as well as their own polarisation in unpolarised nucleons. The latter in particular generates observable azimuthal asymmetries in the final state. These are related to another TMD PDF of great interest, the Boer-Mulders function [67]: it describes the correlation between the quark transverse spin and its transverse momentum in an unpolarised hadron, and it might help explain the well-known violation of the Lam-Tung relation [68] in unpolarised DY reaction [69]. Its counterpart for the gluon content is the distribution of linearly polarised gluons in unpolarised protons which affects, for instance, the $H^{0}$ transverse-momentum distribution at the LHC. At AFTER@LHC, such a distribution can be probed and extracted, for instance, via pseudo-scalar quarkonium production [70,71] and associated quarkonium production [72,73], whereas the quark Boer-Mulders functions can be accessed in DY-pair production.

It is important to note that all these measurements $\left(A_{N}\right.$, azimuthal asymmetries or transverse-momentum spectra) have to be measured in $p p$ collisions as mandatory complementary pieces of information to analogous studies in lepton-induced reactions. Indeed, contrary to usual PDFs, the TMDs are not universal. For instance, some, as the quark Sivers function, are predicted to change sign when generating the STSA in SIDIS and in DY reactions. In other words, the time-reversal odd TMD distributions are process-dependent, but the process dependence is calculable (from the symmetry properties of the theory). This feature, sometimes referred to as a generalised universality, is driven by the nature of the re-scatterings generating the STSA, which can be from the initial or final states. More technically, they are connected to the gauge link in the definition of the TMDs (see [74-76] for recent references). This "sign change" represents one of the most important predictions of the TMD factorisation and dedicated experiments have been proposed to check it [77-80]. Such a process dependence is also explicit in the CT3 formalism. Recently the first measurement in DY was performed by the COMPASS collaboration [81] and the first one on $W$ bosons by the STAR collaboration [79], hinting at the sign change. With AFTER@LHC, one could go further than the current proposals and perform quantitative tests of this generalised universality, deeply connected to the symmetries of QCD. AFTER@LHC will be a unique place to probe such aspects of the parton transverse dynamics in the gluon sector, which requires even more experimental inputs both from lepton- and

\footnotetext{
5 Recently, it has been found that STSA for pion production in proton-proton collisions may be dominantly driven by three-parton fragmentation functions $[42,43]$.
} 


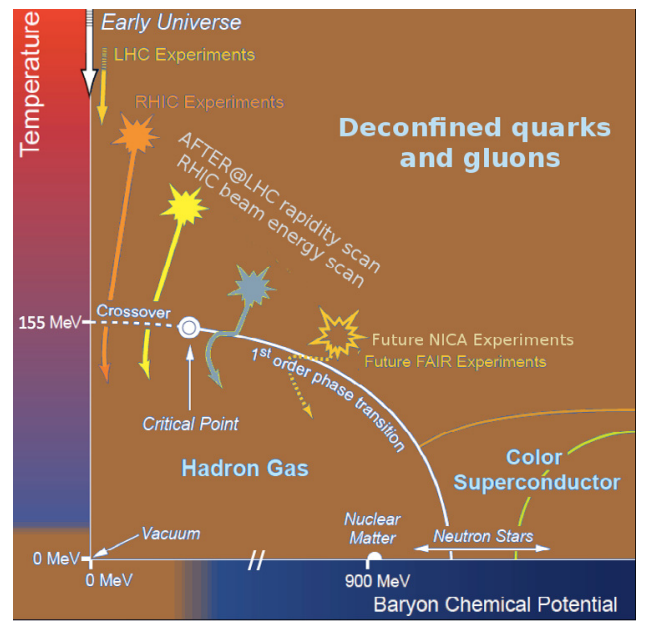

(a)

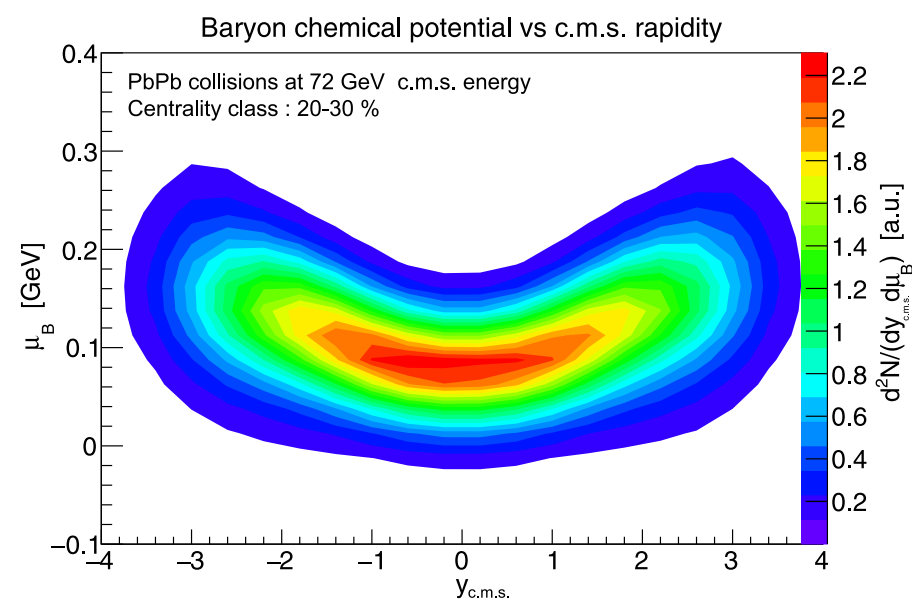

(b)

Fig. 5. (a) Phase diagram of the strongly interacting matter and the reach of AFTER@LHC HI programme. (b) The baryonic chemical potential $\mu_{B}$ as a function of the rapidity $y_{\mathrm{cm} . \mathrm{s}}$ in mid-central PbPb $\sqrt{{S_{N N}}}=72 \mathrm{GeV}$ collisions predicted by the viscous hydro+cascade model vHLLE+UrQMD [Adapted from [86]]. The colour represents the differential density of produced particles as a function of $y_{\text {c.m.s. }}$ and $\mu_{B}$.

hadron-induced reactions than the quark sector. AFTER@LHC will also provide a unique playground to explore in detail the connection between the TMD and CT3 approaches [38,82-85], in particular in the gluon sector. This will open the way for a full three-dimensional mapping of the parton momentum and, in turn, for more insights on the orbital angular momentum of the quarks and gluons.

\subsection{The nuclear matter in new rapidity and energy domains}

One of the prime objectives of Heavy-Ion (HI) physics at high-energy facilities is the search and characterisation of a novel state of matter where the quark and the gluons are deconfined. In this state, which should be the prevailing one of the Universe a few microseconds after the Big Bang, quarks and gluons roam nearly freely over distances of a few femtometers, i.e. distances much larger than the hadron sizes in which they are normally confined.

The existence of such a state of matter is a natural consequence of the asymptotic freedom property of QCD, whereby the strong interaction becomes weak at small distances and high momentum transfers. It is expected to be reached when the surrounding hadronic matter is extremely compressed or heated - resulting in high momentum transfers in the system. These conditions can be achieved in ultra-relativistic collisions of nuclei and the resulting new phase can be observed using specific probes. Such probes are essentially of three kinds, namely radiated particles from the plasma itself (i.e. photons), the destruction of heavy-quark bound states and the momentum-spectrum modification of various particles.

Fig. 5(a) shows the most up-to-date phase diagram of the strongly-interacting matter, gathering all our knowledge that was progressively acquired since the very first relativistic heavy-ion collisions - nearly thirty years ago. With the LHC multi-TeV heavy-ion beams (for now, lead and xenon ions with an energy per nucleon of 2.76 TeV), AFTER@LHC with a c.m.s. energy of $72 \mathrm{GeV}$ provides a complementary coverage to the RHIC- and SPS-based experiments in the region of high temperatures and low baryonic chemical potentials where a Quark-Gluon Plasma (QGP) is expected to be produced. Moreover, model calculations indicate that the baryonic chemical potential $\mu_{B}$ and the temperature $T$ depend on the rapidity [86-88]. Fig. 5(b) shows an example of such a $\mu_{B}$ vs. $y_{\text {c.m.s. }}$ relation for mid-central PbPb $\sqrt{s_{N N}}=72 \mathrm{GeV}$ collisions by the vHLLE+UrQMD model [86]. Measurements conducted as a function of $y_{\text {c.m.s. }}$ will give access to different $\mu_{B}$ and $T$ values. As such, the AFTER@LHC HI programme can rely on a "rapidity scan" to study both the deconfined regime and the expected phase transition to the hadronic gas. It would be a new approach to investigate the QCD phase diagram, complementary to the RHIC Beam Energy Scan (BES) programme [89,90].

Let us also recall here a key advantage of the fixed-target mode, namely the possibility to study different colliding systems with short transition periods while keeping high collected luminosities. ${ }^{6}$ Another key advantage is the obvious

\footnotetext{
6 At the collider LHC, only 4 colliding systems have been studied during Run 1 and 2: pp, pPb, XeXe and PbPb. Even though the RHIC complex is more flexible in this regard, only $p p, d A u, C u C u$ and $\mathrm{AuAu}$ collisions could be studied in the first 10 years of running with enough statistics to study heavy-flavour production. Upgrades were for instance needed to look at $\mathrm{CuAu}, \mathrm{UU}, \mathrm{pAl}, \mathrm{pAu}$ or ${ }^{3} \mathrm{HeAu}$ collisions. In comparison, the LHCb SMOG system (see Section 3.3.1) - which could only so far take data in small periods - already collected data for 5 systems ( $p \mathrm{He}, p \mathrm{Ar}, p \mathrm{Ne}$, PbAr and $\mathrm{PbNe}$ ).
} 


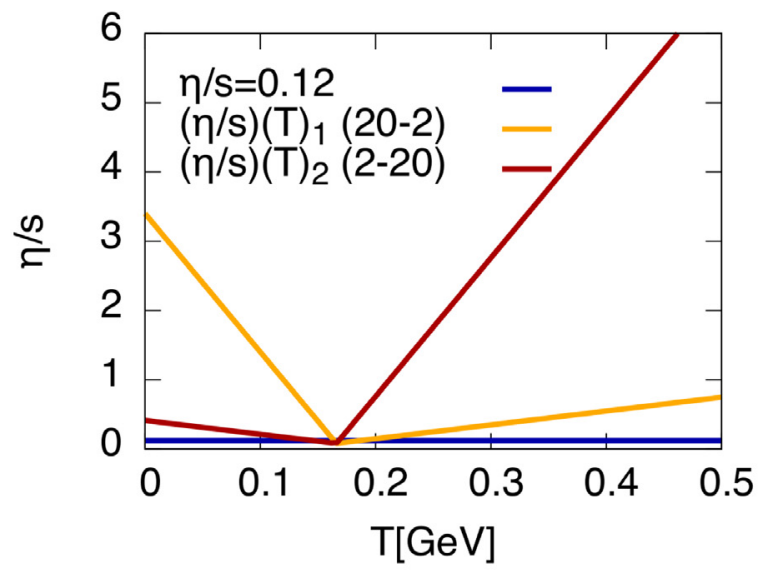

(a)

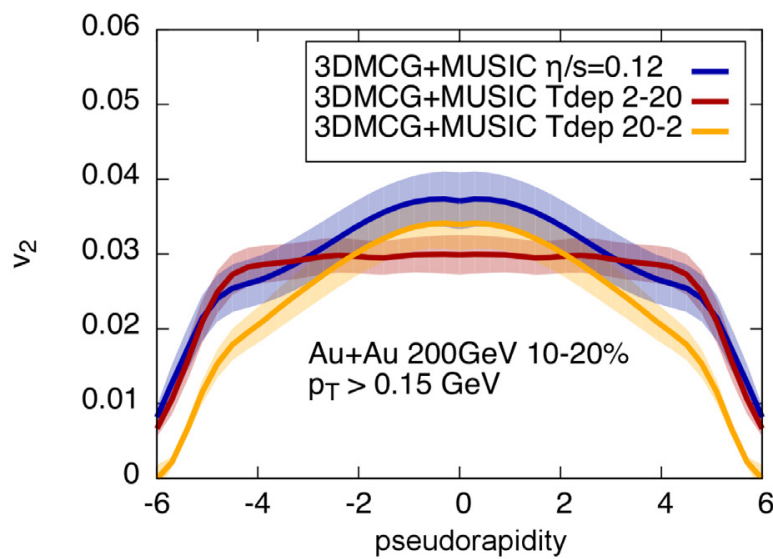

(b)

Fig. 6. (a) Different scenarios of the temperature dependence of the ratio of the medium shear viscosity to the entropy density $(\eta / s)$ at $\mu_{B}=0$; (b) Sensitivity of the second Fourier coefficient $\left(v_{2}\right)$ of the azimuthal asymmetry measured as a function of the pseudo-rapidity with $(\eta / s)$ and the temperature given by $3 \mathrm{D}+1$ viscous hydrodynamic calculations.

Source: Adapted from [92].

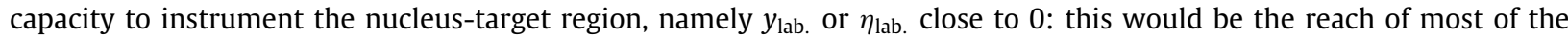
collider detectors used in the fixed-target mode. Based on these assets, the physics case for HI physics with AFTER@LHC can be outlined as follows.

Given the c.m.s.-energy range, HI measurements at AFTER@LHC have the potential to provide us with crucial information about the QGP properties and the nature of the phase transition to the hadronic gas regime. To do so, three experimental degrees of freedom are at our disposal: (i) scanning the longitudinal extension of this hot medium, (ii) colliding systems of different sizes and (iii) analysing the centrality dependence of these collisions. To our knowledge, no other experimental programme could fully rely on these three variables. Together, they should give us a unique lever arm to probe the hot medium at different enough temperatures and energy densities. They will allow one, for instance, to probe the temperature dependence of the shear viscosity to entropy density ratio $(\eta / s)$ of the created matter by measuring the rapidity dependence of the anisotropic flow, as it was recently shown in [91] (see Fig. 6).

More generally, one can perform a tri-dimensional tomography of the hot medium produced in ultra-relativistic HI collisions at $\sqrt{s_{N N}}=72 \mathrm{GeV}$. Such a tomography will rely on a set of precise measurements in a kinematical region ( $y_{\text {c.m.s. }}$ and $\sqrt{s_{N N}}$ ) which could so far only be studied with low accuracy and with an extremely limited set of probes [93,94]. They will give us new information on the QGP properties in the longitudinal dimension and help us settle long-standing debates about probes such as heavy-quark(onium) production in the range from SPS to RHIC energies [95-97]. These issues admittedly hinder their potential as golden probes of the QGP [98].

Measurements at $y_{\text {c.m.s. }} \simeq 0$ are also at reach with detector coverages about $\eta_{\text {lab. }} \simeq 4$.0. Studying an extensive set of hard probes in this region, where the yields are the highest, is meant to provide us with a very reliable calibration of the properties of the system (temperature, viscosity) in order to initiate the scan in the longitudinal direction via the rapidity dependence.

\section{How to make fixed-target collisions at the LHC?}

\subsection{Overview}

Several technological options are currently under investigation to perform dedicated fixed-target experiments at the LHC. One can indeed initiate collisions of the LHC beam particles with nucleons or nuclei at rest:

- by letting the full LHC beam go through a gas target in the LHC beam pipe,

- by extracting halo particles by means of a bent-crystal deflector onto a target positioned outside the beam pipe with a dedicated beam line or inside the beam pipe,

- or by placing a wire target intercepting the faint beam halo in the beam pipe.

The aim of this section is to summarise the advantages, the performances as well as the challenges of each solution. We first discuss them and then compare their performances and limitations.

The LHCb collaboration with its SMOG [99] and VELO [100] systems has demonstrated [101] that gas injection within a certain range is tolerable and does not lead to vacuum instabilities thus paving the way towards a genuine gas target 
Table 1

LHC-related quantities used in the calculations in the following sections along the expected parameters for an upgraded Pb beam mode. The useable particle flux in the halo is assumed to be half of the estimated beam losses in the proton and lead beams. The LHC yearly running times quoted are maxima.

\begin{tabular}{lll}
\hline & Proton beam & Lead beam \\
\hline Number of bunches in the LHC $\left(N_{b}\right)$ & 2808 & 592 \\
Number of particles per bunch $\left(N_{p}\right)$ & $1.2 \times 10^{11}$ & $7.0 \times 10^{7}$ \\
LHC revolution frequency $(v)[\mathrm{Hz}]$ & & 11245 \\
Particle flux in the LHC $\left(\phi_{\text {beam }}\right)\left[\mathrm{s}^{-1}\right]$ & $3.6 \times 10^{18}$ & $4.7 \times 10^{14}$ \\
LHC yearly running time $(\Delta \mathrm{t})[\mathrm{s}]$ & $10^{7}$ & 7 \\
Nominal energy of the beam $\left(\mathrm{E}_{\text {beam }}\right)[\mathrm{TeV}]$ & 10 & $2.70^{6}$ \\
Fill duration considered $(\Delta \tau)[\mathrm{h}]$ & $5 \times 10^{15}$ & 5 \\
Useable particle flux in the halo $(\phi$ useable halo $)\left[\mathrm{s}^{-1}\right]$ & $10^{6}$ & 2.76 \\
\hline
\end{tabular}

with the polarisation of light nuclei with higher densities, as well as for heavy noble gases. It allows one to use an existing LHC detector, resulting in limited costs and a relatively short time-scale installation. As we will discuss in the following sections, the acceptances of the ALICE and LHCb detectors are, in general, well suited for data taking in the fixed-target mode.

A long narrow tube - commonly referred to as a storage cell [102] - placed on-axis of the LHC beam and fed by a polarised or unpolarised gas clearly presents an interesting opportunity to reach high luminosities for the gas-target option (see Section 3.3.3). Such a configuration allows for different beam-target combinations including highly polarised ones - pending the cell-coating compatibility with the LHC vacuum constraints. The possibility for a density calibration of the storage-cell targets has still to be studied. If successful this would, together with the known LHC beam current, allow one to measure absolute luminosities and cross sections. For a given (areal) target density, the storage cell requires the lowest - but still sizeable - gas flow into the LHC vacuum system.

Alternatively, higher gas fluxes can directly be injected orthogonally to the beam (see Section 3.3.2). This is how the RHIC H-jet polarimeter [103], with (highly) polarised injected hydrogen, operates. In general, this leads to lower luminosities than the storage-cell option. However, if used in conjunction with the ALICE detectors, the luminosities obtainable with a gas-jet system would already be reaching the limit of the detector data-taking capabilities. In the case of polarised ${ }^{3} \mathrm{He}$ and other unpolarised gases, more intense sources can in principle be used to compensate for the smaller target-areal density. The advantage of this option is the very high reachable polarisation of the target and the limited need of R\&D for an installation in the LHC complex.

On the other hand, bent crystals are being developed as part of the collimators protecting the machine [104]. They may also be placed near the beam in order to deflect halo particles and guide them onto an external target (see Section 3.5.1). Beside thick unpolarised targets, cryogenic polarised targets could be employed. This approach involves a considerable amount of civil engineering, including a new cavern and a new detector. Another possibility based on such a bent crystal is to directly use the deviated particles of the halo on an internal target system, inside the beam pipe of an existing LHC experiment. The feasibility of such a solution is currently explored by the UA9 collaboration at CERN [105]. Open problems in this case are how to dump the deflected beam, and how to get a suitable polarised target.

Finally, a wire or foil target may be placed in the halo of the LHC beam [106] in order to provide collisions at a near-axis position (see Section 3.4). This method has been employed at HERA-B [107] and STAR [108] and is particularly useful for heavy-nucleus targets. However, it may affect the main LHC beam and this solution will require dedicated simulations. In addition, it is probably not compatible with light-nucleus targets - certainly not hydrogen ones - and basically prevents one to perform any direct luminosity measurement.

In the next sections, the aforementioned options are detailed. In Section 3.6, the Figure-of-Merit (FOM) for collisions with unpolarised and polarised targets is defined and numerical values are given, allowing for a comparison of performances. A qualitative summary table of the performances of the various technological solutions to initiate fixed-target collisions at LHC is also discussed. Finally, expected integrated luminosities for each solutions are compared.

\subsection{Relevant LHC parameters and definitions}

Let us first recall (in Table 1) some nominal LHC parameters $[109,110]$ as well as other generic quantities which have been used in the comparison of the various technological solutions.

Beside these nominal running conditions, the possibility of $p$ runs at the Pb energy for reference measurements is also possible as already done during the Runs 1 and 2 . These runs typically last one week, i.e. $\mathcal{O}\left(10^{5}\right)$ seconds. When relevant, these run durations will be specified. We also note that other beam species $(\mathrm{Kr}, \mathrm{Ca}, \mathrm{O})$ can be considered for an injection in the LHC (in particular those used in the SPS, i.e. Xe and Ar), as it was the case with the short XeXe run which took place by the end of 2017. The injection of other species, like He, would require another ion source than the current one. In most cases, the instantaneous luminosities should be equal or larger than those for Pb beams (see later).

Let us now recall useful quantities to compare the various technological implementations. First, we define the

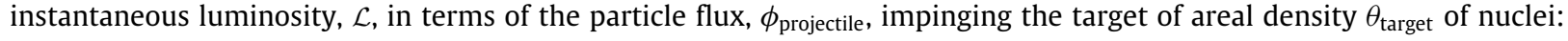

$$
\mathcal{L}=\phi_{\text {projectile }} \times \theta_{\text {target }}
$$


If $\theta_{\text {target }}$ is expressed in atoms $\times \mathrm{cm}^{-2}, \mathcal{L}$ is naturally expressed in $\mathrm{cm}^{-2} \mathrm{~s}^{-1}$. For a gas target, the flux is $\phi_{\text {beam }}$, the particle flux in the LHC. For a solid wire target or a solid target put into an extracted beam, it is $\phi_{\text {useable halo, }}$, the useable particle flux in the halo, which is on the order of the beam losses (or less) to allow for a parasitic-working mode.

To compare the performances of the target for spin-related measurements, in particular Single-Spin Asymmetries (SSAs) discussed in Section 5.2, we define $\mathcal{F}$, the spin figure of merit of the target (and beam):

$$
\mathcal{F}=\mathcal{P}_{\text {eff }}^{2} \times \mathcal{V} .
$$

where $\mathcal{P}_{\text {eff, }}$ which we call the effective polarisation of the target, contains the information about the polarisability of the material and $\mathcal{V}$ contains the information about the rates (up to the cross section of the considered reaction). It is important to understand that our comparisons will involve different target systems and materials, with different beam fluxes. A figure of merit only accounting for the target properties is therefore not sufficient.

Depending on the target material type, $\mathcal{P}_{\text {eff }}$ can be expressed as:

$$
\mathcal{P}_{\text {eff }}=P_{T} \times f \text { or } \mathcal{P}_{\text {eff }}=P_{T} \times \alpha,
$$

where $P_{T}$ is the polarisation of the nucleons in the target, $f$ a dilution factor and $\alpha$ a depolarisation factor. The dilution factor $f$ is in principle defined as the fraction of events scattered off the polarisable nucleons in the target. As such, it can be approximated to the fraction of the polarised nucleons in the molecules constituting the target. However, whereas this approximation is sufficient to compare similar systems, it may not encompass nuclear or isospin effects which depend on the kinematics or the probe to be studied. Following the former definition, one has [111]:

$$
f=\frac{n_{N} \sigma_{N}}{n_{N} \sigma_{N}+\sum_{i} n_{A_{i}} \sigma_{A_{i}}},
$$

where $n_{N, A}$ are respectively the number of polarised nucleons and of nuclei $A$ in the target. $\sigma_{N, A}$ are the corresponding cross sections for the considered observables. Because of nuclear effects, we know that $\sigma_{A}=A \times \sigma_{N}$ does not hold in general and approximating $f$ to the fraction of polarisable nucleons is not strictly correct. In the case of DIS at large $x$, the variation of such corrections arises from the EMC effect which results in visible variations of $f$ [111] as a function of $x_{B}$. As a matter of fact, the experimental derivation of $f$ amounts to measuring the cross sections off the different constituents of the target. When this is not possible, such ratios can be evaluated based on theoretical computations. In the following, we will only refer to an average value, denoted $\langle f\rangle$, computed from the fraction of polarisable nucleons.

Since the precision of a given measurement does depend on the counting rates, it is important to realise that the luminosity $\mathcal{L}$ does not account for the number of the nucleons per atom or molecule in the target, but instead for that of the nuclei. For instance, at the LHC in the collider mode, similar yields are obtained in $p p$ and $p \mathrm{~Pb}$ collisions when the luminosity for the $p p$ case is about 200 times larger than that of the $p \mathrm{~Pb}$ case - thus not at all at similar luminosities. This should in principle be reflected in a figure of merit. However, the luminosity is not a good one for the counting rates when different colliding systems are considered.

In the case of "rare" or "hard" processes (such as quarkonium, heavy-quark or DY pair production), each nucleon approximatively contributes additively $\left(\sigma_{A} \sim A \times \sigma_{p}\right)$ and $\mathcal{V}$ can be expressed as:

$$
\mathcal{V}=\mathcal{L} \times \sum_{i} A_{i}
$$

where $\sum_{i} A_{i}$ is the sum of the atomic masses of the target constituents (for instance 1 for $\mathrm{H}, 3$ for ${ }^{3} \mathrm{He}$ and 17 for $\mathrm{NH}_{3}$ ). ${ }^{7}$ In the case of SSAs of light hadrons, where the yields do not follow the binary-collision scaling in a single nucleus $\left(\sigma_{A}\right.$ strongly deviates from $A \times \sigma_{p}$ ), or for collisions induced by a nuclear beam, there is no simple equivalent for $\mathcal{V}$ and the rates should be computed in order to perform meaningful comparisons between polarised targets made of different species. It is of course also the case if the beam energy happens to be significantly different such as to induce very different production cross sections.

Only for hard probes and when nuclear effects can be neglected, it follows that $\mathcal{F}$ defined as above is inversely proportional to the time needed to reach a fixed precision on SSAs. In other words $\mathcal{F}$ is inversely proportional to the variance of the SSAs.

Since different set-up cannot be used during the same amount of time over a LHC year, it is also obviously useful to make comparisons at the level of $\int d t \mathcal{L}$ and $\int d t \mathcal{F}$. The yearly run duration is taken to be $10^{7} \mathrm{~s}$ for the proton beam and $10^{6} \mathrm{~s}$ for the lead beam. These are maxima.

\subsection{Internal gas-target solutions}

\subsubsection{SMOG: a feasibility demonstrator}

The direct injection in the LHC beampipe (i.e. in the VELO vessel) of noble gases at a pressure on the order of $10^{-7}$ mbar is already being used by the LHCb collaboration with the SMOG (System for Measuring the Overlap with Gas)

\footnotetext{
7 Note that $\sum_{i} A_{i}$ does not reduce to the sum of the atomic masses of the target constituents when $\theta_{\text {target }}$ is expressed in atoms per $\mathrm{cm}^{2}$ for molecular gases, such as $\mathrm{H}_{2}$.
} 


\section{Table 2}

Beam type, target type, gas pressure $(P)$, useable gas length $(\ell)$, target areal density $\left(\theta_{\text {target }}\right)$, instantaneous luminosity $\left(\mathcal{L}=\phi_{\text {beam }} \theta_{\text {target }}\right)$, hadronic beam-target cross section ( $\left.\sigma_{\text {beam-target }}^{\text {had }}\right)$ and beam fraction lost over a fill $(b)$ for a SMOG-like device. The areal density can be expressed for perfect gases as $\theta_{\text {target }}\left(\mathrm{cm}^{-2}\right)=P \ell \mathcal{N}_{\mathcal{A}} / 22697$ where $P$ is in bar, $\ell$ is in $\mathrm{cm}$ and $\mathcal{N}_{\mathcal{A}}$ is the Avogadro number in mol ${ }^{-1}$. The useable gas zone assumed is $\ell=40 \mathrm{~cm}$. We considered the nominal parameters from Table 1 for the beam energy, the particle flux in the LHC and the fill duration. The obtained instantaneous luminosities should therefore be considered as maxima. The hadronic cross-sections used in this section to compute the beam fraction lost over a fill have been obtained using the EPOS MC generator [114]. The values are compatible with those from Fluka generator [115] within 6\%.

\begin{tabular}{|c|c|c|c|c|c|c|c|}
\hline Beam & Target & $\begin{array}{l}P \\
{[\text { mbar }]}\end{array}$ & $\begin{array}{l}\ell \\
{[\mathrm{cm}]}\end{array}$ & $\begin{array}{l}\theta_{\text {target }} \\
{\left[\text { atoms } \times \mathrm{cm}^{-2} \text { ] }\right.}\end{array}$ & $\begin{array}{l}\mathcal{L} \\
{\left[\mathrm{cm}^{-2} \times \mathrm{s}^{-1}\right]}\end{array}$ & $\begin{array}{l}\sigma_{\text {beam-target }}^{\text {had }} \\
\text { [barn] }\end{array}$ & $\begin{array}{l}b \\
{[\%]}\end{array}$ \\
\hline$p$ & $\{\mathrm{He}, \mathrm{Ne}, \mathrm{Ar}, \mathrm{Kr}, \mathrm{Xe}\}$ & $1.5 \times 10^{-7}$ & 40 & $1.6 \times 10^{11}$ & $5.8 \times 10^{29}$ & $\{0.2,0.6,1.0,1.6,2.2\}$ & $\ll 0.1$ \\
\hline $\mathrm{Pb}$ & $\{\mathrm{He}, \mathrm{Ne}, \mathrm{Ar}, \mathrm{Kr}, \mathrm{Xe}\}$ & $1.5 \times 10^{-7}$ & 40 & $1.6 \times 10^{11}$ & $7.4 \times 10^{25}$ & $\{4.5,7.0,8.4,10,12\}$ & $\ll 0.1$ \\
\hline
\end{tabular}

device. Initially developed inside $\mathrm{LHCb}$ to allow for a precise determination of the luminosity with an uncertainty below $4 \%$, SMOG is a system whereby a gas can be injected inside the beam vacuum at the interaction point. The luminosity for the collider mode is then determined thanks to a Beam Gas Imaging (BGI) method, which relies on the interaction vertices between the circulating beam and the gas present at the interaction point $[99,112,113]$.

The system and its technology have been extensively tested. A pilot run of $p$ beam (Pb beam) on a Neon gas target was successfully performed in 2012 (2013) at a c.m.s energy of $\sqrt{s_{N N}}=87 \mathrm{GeV}(54 \mathrm{GeV})$. This first SMOG campaign was followed by several successful data taking periods in 2015-2017, for which the injection of other gases than Neon was explored. The system is currently limited to noble gases, namely $\mathrm{He}, \mathrm{Ne}, \mathrm{Ar}$ and, possibly, to $\mathrm{Kr}$ and Xe which have not yet been tested. Their impact on the machine is currently under discussion with the LHC vacuum experts. The limitation to noble gas is to avoid altering the Non-evaporable-getter (NEG) coating properties of the beam pipe and this is why SMOG is equipped with a NEG cartridge to ensure the purity of injected gas. These noble gases can thus travel from the injection point (IR8) to the ion pump stations at $\pm 20 \mathrm{~m}$ and some can reach the warm-to-cold transitions of the Q1 magnets, where they can accumulate during extended periods of injection. The beam-induced effects due to gas cryosorbed on those surfaces are still being investigated along the successive tests. However, in 2015, Ar was injected in LHCb for about one week in a row, during the heavy-ion run which took place before the Year End Technical Stop (YETS). No decrease of the LHC performances was observed. It therefore opens good perspectives for data taking periods of up to a month per year without additional pumping systems. It would preferably take place at the end of the year, while the beam intensity is low, and before the YETS which could permit to get rid of the accumulated gas if needed. With the current SMOG set-up, the gas pressure is about $1.5 \times 10^{-7} \mathrm{mbar}$, i.e. two orders of magnitude higher than the LHC vacuum pressure $\left(\sim 10^{-9}\right.$ mbar). The pressure might be increased up to $10^{-6}$ mbar without severe hardware changes along with the support of the LHC vacuum experts. An estimate of the instantaneous luminosity obtained with the SMOG device in p-gas and Pb-gas collisions is given in Table 2 .

The beam fraction consumed over a fill is negligible. There are prospects to replace SMOG with a multigas system allowing to change the type of injected gas without human intervention onsite. As for now, the gas pressure is not well known with a good precision. The installation of a calibrated Vacuum Gauge Ion (VGI) $6 \mathrm{~m}$ from the VELO has been performed during the YETS of 2016-2017. The luminosity is estimated by the parameters of the beam (number of bunches and bunch intensity), as well as the gas-target pressure measured by the pressure gauge. The uncertainty is dominated by the measured pressure, which varies as a function of the $z$ position along the beam. The luminosity is also determined from the yield of electrons scattering off the target atoms, with a precision of about 6\% [116]. In addition to the luminosity determination challenge, it is also worth noting that one has to cope with colliding-bunch events if the detector is also used in the collider mode. Fixed-target heavy-flavour analyses are currently limited to the analyses of non-colliding bunch events, with a vertex position within -20 and $20 \mathrm{~cm},{ }^{8}$ i.e. in a region where the detector efficiencies are mostly constant with respect to the $z$-vertex position [117]. Such requirements affect the effective recorded luminosities. One has to keep in mind that besides the limitation of the SMOG system itself in terms of the delivered gas pressure, the contamination of the collider events by fixed-target events also has to be considered as a constraint on the maximum pressure which can be delivered to LHCb. This consideration applies only in parasitic operation mode (during $p p$ runs), in order not to affect the current main LHCb B physics programme.

\subsubsection{Gas-jet target}

Out of all the proposed solutions, the internal gas-target cell is a genuine solution to make fixed-target experiments at the LHC, at limited cost by re-using an existing LHC experiment, and with a variety of polarised and unpolarised gas targets. From the experience gained with SMOG, and further tests of gas injection in Long Straight Section 4 (LSS4) and near ALICE, ATLAS and CMS, we consider the option of direct-injection of gas in the LHC beampipe as a viable solution to be run parallel to the collider mode. As highlighted above, the SMOG system is however not optimised and faces some limitations: the gas pressure - thus the target density - is limited, only noble gases can be injected, the heavier noble

8 The vertex requirement is extended to $-70<z<10 \mathrm{~cm}$ in [116]. 
Table 3

Beam type, target type, inlet flux $\left(\varphi_{\text {target }}^{\text {inlet }}\right)$, target areal density $\left(\theta_{\text {target }}\right)$, instantaneous luminosity $\left(\mathcal{L}=\phi_{\text {beam }} \theta_{\text {target }}\right)$, hadronic beam-target cross section $\left(\sigma_{\text {beam-target }}^{\text {had }}\right)$ and beam fraction lost over a fill $(b)$ for a gas-jet target inspired from the RHIC proton-beam polarimeter. We considered the nominal parameter from Table 1 for the beam energy, the particle flux in the LHC and the fill duration. The hadronic cross-sections are obtained as described in Table 2. The obtained instantaneous luminosities should be considered as maxima. We have however levelled the gas inlet flux such that the lifetime of the beam is not shorten by an unrealistically large amount (we have considered $15 \%$ ).

\begin{tabular}{|c|c|c|c|c|c|c|}
\hline Beam & Target & $\begin{array}{l}\varphi_{\text {target }}^{\text {inlet }} \\
{\left[\text { atoms } \times \mathrm{s}^{-1}\right]}\end{array}$ & $\begin{array}{l}\theta_{\text {target }} \\
{\left[\text { atoms } \times \mathrm{cm}^{-2} \text { ] }\right.}\end{array}$ & $\begin{array}{l}\mathcal{L} \\
{\left[\mathrm{cm}^{-2} \times \mathrm{s}^{-1}\right]}\end{array}$ & $\begin{array}{l}\sigma_{\text {beam-target }}^{\text {had }} \\
\text { [barn] }\end{array}$ & $\begin{array}{l}b \\
{[\%]}\end{array}$ \\
\hline$p$ & $\mathrm{H}^{\uparrow}$ & $1.3 \times 10^{17}$ & $1.2 \times 10^{12}$ & $4.3 \times 10^{30}$ & $48 \times 10^{-3}$ & $\ll 0.1$ \\
\hline$p$ & $\mathrm{D}^{\uparrow}$ & $1.3 \times 10^{17}$ & $1.2 \times 10^{12}$ & $4.3 \times 10^{30}$ & $90 \times 10^{-3}$ & $\ll 0.1$ \\
\hline$p$ & ${ }^{3} \mathrm{He}^{\uparrow}$ & $1.0 \times 10^{19}$ & $1.0 \times 10^{14}$ & $3.6 \times 10^{32}$ & $12 \times 10^{-2}$ & 0.5 \\
\hline$p$ & $\mathrm{H}_{2}$ & $1.1 \times 10^{20 \div 21}$ & $1.0 \times 10^{15 \div 16}$ & $3.6 \times 10^{33 \div 34}$ & $48 \times 10^{-3}$ & $1.9 \div 19$ \\
\hline$p$ & $\mathrm{Xe}$ & $(1.0 \div 5.0) \times 10^{18}$ & $(1.0 \div 5.0) \times 10^{13}$ & $(3.6 \div 18) \times 10^{31}$ & 2.2 & $0.9 \div 4.4$ \\
\hline $\mathrm{Pb}$ & $\mathrm{H}^{\uparrow}$ & $1.3 \times 10^{17}$ & $1.2 \times 10^{12}$ & $5.6 \times 10^{26}$ & 3.0 & $\sim 0.1$ \\
\hline $\mathrm{Pb}$ & $\mathrm{D}^{\uparrow}$ & $1.3 \times 10^{17}$ & $1.2 \times 10^{12}$ & $5.6 \times 10^{26}$ & 4.0 & $\sim 0.1$ \\
\hline $\mathrm{Pb}$ & ${ }^{3} \mathrm{He}^{\uparrow}$ & $1.0 \times 10^{19}$ & $1.0 \times 10^{14}$ & $4.7 \times 10^{28}$ & 4.3 & 8.7 \\
\hline $\mathrm{Pb}$ & $\mathrm{H}_{2}$ & $6.5 \times 10^{16}$ & $2.5 \times 10^{14}$ & $1.2 \times 10^{29}$ & 3.0 & 15 \\
\hline $\mathrm{Pb}$ & $\mathrm{Xe}$ & $(1.0 \div 5.0) \times 10^{18}$ & $(1.0 \div 5.0) \times 10^{13}$ & $(0.5 \div 2.3) \times 10^{28}$ & 12 & $2.6 \div 12$ \\
\hline
\end{tabular}

gases may only be used before long YETS and the injection periods are currently significantly limited. Last but not least, a SMOG-like system does not allow one to inject polarised gases.

Some of these limitations can be lifted by the installation of specific pumping systems, which would however reduce the portability of the system. ${ }^{9}$ In addition, a specific injection system would allow one to inject heavier noble gases as well as a jet of polarised gases such as $\mathrm{H}, \mathrm{D}$ and ${ }^{3} \mathrm{He}$. One illustrative example of such an option is the H-jet system [103] used on the BNL-RHIC collider as a proton-beam polarimeter (see Appendix A.1). In short, it offers much higher target densities than the current SMOG system, opens the possibilities for highly polarised target and can be coupled to a collider.

Let us briefly describe its main characteristics: it consists of a free atomic beam vertically crossing the collider beam at a speed of approximately $1560 \mathrm{~m} \mathrm{~s}^{-1}$. With the current Atomic Beam Source (ABS) [103], operated at $70 \mathrm{~K}$, the polarised $\mathrm{H}$ inlet flux was measured to be $(1.24 \pm 0.02) \times 10^{17} \mathrm{H}^{\uparrow} \mathrm{s}^{-1}$. With a redesigned system, it may be doubled [118]. Similar numbers should be reachable for a polarised deuterium. Higher fluxes can easily be obtained with polarised ${ }^{3} \mathrm{He}$ but would require a dedicated system [118]. Using a deuterium target would require proper RF cavities which may prevent optimising both hydrogen and deuterium target performances with a single system.

At the interaction point, the H-jet target profile is nearly Gaussian with a full width at half maximum of $5.5 \mathrm{~mm}$. This is a significant advantage compared to a SMOG-like system where the gas diffuses along the beampipe and results in beam-gas collisions over distances of a few metres. A vacuum in the RHIC ring of $2 \times 10^{-8}$ mbar was achieved with the $\mathrm{H}$-jet system in operation at its nominal $10^{17}$ atoms s${ }^{-1}$ inlet flux thanks to turbomolecular pumps (TMP) with a $1000 \mathrm{l} / \mathrm{s}$ pumping speed. The current H-jet system size is $375 \mathrm{~cm}$ high $(225 \mathrm{~cm}$ for ABS above and $150 \mathrm{~cm}$ for Breit-Rabi polarimeter below the beampipe), $110 \mathrm{~cm}$ wide and $70 \mathrm{~cm}$ long. A redesigned system could be made more compact down to 200 (75) $\mathrm{cm}$ high above (below) the beam pipe. The current free ABS corresponds to a target areal density of $(1.2 \pm 0.2) \times 10^{12}$ $\mathrm{H}^{\uparrow} \mathrm{cm}^{-2}$.

The H-jet system is designed to be movable. It can be uninstalled and reinstalled in 2-3 days. It is coupled to a BreitRabi polarimeter monitor to measure with a high accuracy the atomic hydrogen polarisation which is as high as 0.96 . The gas jet is however contaminated by hydrogen atoms bound into unpolarised proton molecules which slightly dilutes the average jet-proton polarisation down to $P^{\mathrm{H}-\mathrm{jet}}=93 \%$ [119].

It was recently demonstrated [120] that the molecular hydrogen atomic mass fraction is about $0.4 \%$ in the jet centre. However, since the molecular-hydrogen distribution is a factor 30-40 wider than the jet one, an integral dilution of the jet polarisation may be as large as 0.85 . By detecting recoil protons from elastic pp scatterings, it was possible to reconstruct the $z$-coordinate of the vertex which allowed the in-situ normalisation of the molecular-hydrogen contribution and its proper subtraction. For the actually used event-selection cuts, the effective jet-target polarisation was found to be $95 \pm 0.5 \%$. To employ this method of the jet-polarisation control, the vertex $z$-coordinate has to be measured with an accuracy of $\sigma_{z} \lesssim 1 \mathrm{~mm}$. We further note [103] that the polarisation could reach 96\%-98\% if coupled with a higher holding magnetic field than the present one of $0.14 \mathrm{~T}$. For the H-jet system, the field strength was also chosen to minimise the bending of the scattered recoil protons for the use as a polarimeter.

The gas-jet parameters, the instantaneous luminosities using typical LHC beam currents, and the beam fraction consumed over a fill are shown in Table 3, for each type of beam-gas collisions.

In particular, an estimation of the target areal density is given for polarised ${ }^{3} \mathrm{He}$ and unpolarised jet targets. In the former case, a higher areal density is achievable since ${ }^{3} \mathrm{He}$, if cooled down, can be injected with lower velocity than $\mathrm{H}$. For what concerns unpolarised heavier ions, a target areal density of $1 \div 5 \times 10^{13} \mathrm{~cm}^{-2}$ can be envisioned [103].

9 A SMOG-like system essentially reduces to a gas bottle, some valves and pressure gauges, a NEG filter and a small capillary to the beam pipe. 


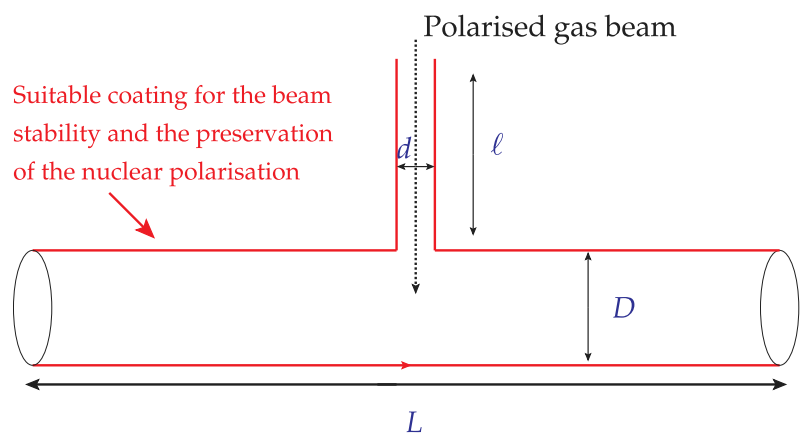

(a)

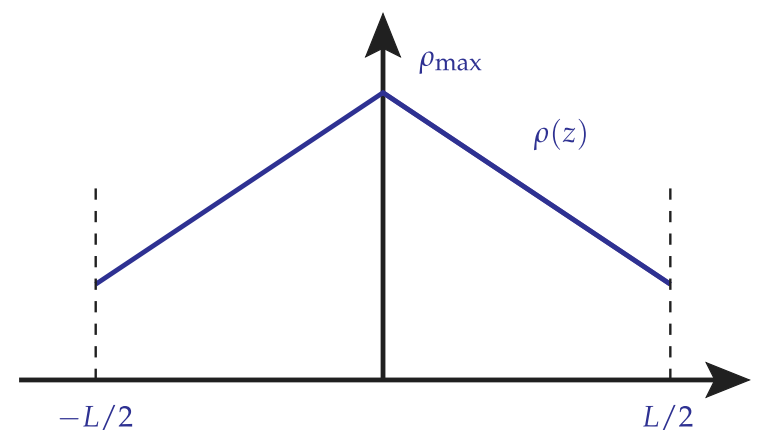

(b)

Fig. 7. (a) Schematic view of the target cell. Polarised gas can be injected ballistically - unpolarised gas via a capillary - into the cell centre; (b) Gas density profile, $\rho(z)$ along the beam $z$ axis.

\subsubsection{Storage-cell gas target}

Besides a gas-jet solution, using an internal gas-target cell is another genuine solution to make fixed-target experiments at the LHC, at a limited cost by re-using existing LHC detectors, and with a variety of polarised and unpolarised gas targets.

In such a case, the target [121] consists of a polarised or unpolarised gas source in combination with an open storage cell (see Fig. 7) which increases the target density by more than two orders of magnitude compared to a free atomic beam jet.

The storage cell consists of a narrow straight tube with thin walls located in the machine vacuum along the beam axis, into which the gas is injected at the centre, in two modes:

- polarised atomic beam (H, D or $\left.{ }^{3} \mathrm{He}\right)$ into a feed tube of low gas conductance;

- unpolarised gas (e.g. $\mathrm{H}_{2}, \mathrm{He}, \mathrm{Ne}, \mathrm{Ar}, \mathrm{Kr}, \mathrm{Xe}$ ) via a capillary from a gas handling system.

The gas diffuses through the cell openings into the machine vacuum system, which usually requires a powerful differential pumping system. The cell consists of two movable halves that can be opened in order to provide space for beam injection or manipulation. Polarised gas targets for storage rings are reviewed in [102]. Targets for proton beams at intermediate energies have been applied at the cooler ring COSY (FZ Jülich) [122-124] as well as at the IUCF Cooler Ring [125]; and for electron beams at HERA (DESY Hamburg) [126].

Such a polarised H or D target (the HERMES target) [126] consists of three main components: the polarised Atomic Beam Source (ABS), the Storage Cell in a longitudinal or vertical holding field $B$ and a Diagnostic System for analysing a small sample of gas from the target cell, consisting of the polarimeter (BRP) measuring the sub-state population of the atoms, and the target gas analyser (TGA) detecting the molecular fraction and thus the degree of recombination within the cell. From these parameters, the target polarisation $P$ as seen by the beam is deduced (typical values $P \approx 0.85$, corrected for magnetic guide field and degree of dissociation). It was however recently noted that the cell coating used then does not comply with the LHC requirements. R\&D is thus needed to find a proper coating which would not significantly degrade the polarisation performances.

The ABS injects polarised $\mathrm{H}$ into the feed tube (here $100 \mathrm{~mm}$ long, $10 \mathrm{~mm}$ inner diameter) of a storage cell of $1000 \mathrm{~mm}$ length with a proposed inner diameter of $14 \mathrm{~mm}$ (see Fig. 7). A smaller size of $300 \mathrm{~mm}$ by $10 \mathrm{~mm}$ however seems to be more adapted to match the LHC requirements [127]. The maximum areal density $\theta_{\text {target }}$ is limited by the flux of the ABS and the geometry of the storage cell which needs to permit the transmission of the LHC beam and the injection of the polarised atomic beam. The target described here is also able to deliver polarised D with similar densities. Injecting optically-pumped polarised ${ }^{3} \mathrm{He}$ into the storage cell could be another possibility. In such a case, the holding field must be very homogeneous in order to prevent motional depolarisation.

Unpolarised target gas could also be injected into the cell via a capillary for the study of heavy-ion collisions. The gas flow from a gas feed system and thus the gas density in a target cell can be very high. Limits are imposed e.g. by the maximum gas flow that a possible LHC target station can take, and by the capability of the detector system. Another requirement is that the gas target must not shorten the lifetime of a $\mathrm{Pb}$ beam fill by an unrealistically large amount (we have considered $\sim 15 \%$ ), when running parasitically. Indeed, higher luminosities could be reached for dedicated runs. On top of hadronic interactions, photo-nuclear interactions including Bound-Free Pair Production, in which an $e^{+} e^{-}$pair is created with the electron bound to one of the colliding nuclei, and Electromagnetic Dissociation [128] can affect the Pb beam lifetime. The photo-nuclear interactions are enhanced with the target gas atomic number and can be up to twenty times the hadronic cross section in case of Xe gas with $\mathrm{Pb}$ beam [129]. The storage cell parameters, the instantaneous luminosities obtained using typical LHC beam currents, and the beam fraction consumed over a fill, considering hadronic interactions, are shown in Table 4, for each type of beam-gas collisions [121]. 


\section{Table 4}

Beam type, target type, inlet flux $\left(\varphi_{\text {target }}^{\text {inlet }}\right)$, target areal density $\left(\theta_{\text {target }}\right)$, instantaneous luminosity $\left(\mathcal{L}=\phi_{\text {beam }} \theta_{\text {target }}\right)$, hadronic beam-target cross section ( $\left.\sigma_{\text {beam-target }}^{\text {had }}\right)$ and beam fraction lost over a fill $(b)$ for a storage cell target with a cell length of $1 \mathrm{~m}$ and temperature of $300 \mathrm{~K}$. We considered the nominal parameter from Table 1 for the beam energy, the particle flux in the LHC and the fill duration. The hadronic cross-sections are obtained as described in Table 2. The obtained instantaneous luminosities should be considered as maxima. We have however levelled the gas inlet flux such that the lifetime of the beam is not shorten by an unrealistically large amount (we have considered $15 \%$ ).

\begin{tabular}{|c|c|c|c|c|c|c|}
\hline Beam & Target & $\begin{array}{l}\varphi_{\text {target }}^{\text {inlet }} \\
{\left[\text { atoms } \times \mathrm{s}^{-1} \text { ] }\right.}\end{array}$ & $\begin{array}{l}\theta_{\text {target }} \\
{\left[\text { atoms } \times \mathrm{cm}^{-2}\right]}\end{array}$ & $\begin{array}{l}\mathcal{L} \\
{\left[\mathrm{cm}^{-2} \times \mathrm{s}^{-1}\right]}\end{array}$ & $\begin{array}{l}\sigma_{\text {beam-target }}^{\text {had }} \\
\text { [barn] }\end{array}$ & $\begin{array}{l}b \\
{[\%]}\end{array}$ \\
\hline$p$ & $\mathrm{H}^{\uparrow}$ & $6.5 \times 10^{16}$ & $2.5 \times 10^{14}$ & $9.2 \times 10^{32}$ & $48 \times 10^{-3}$ & 0.4 \\
\hline$p$ & $\mathrm{D}^{\uparrow}$ & $5.2 \times 10^{16}$ & $2.9 \times 10^{14}$ & $1.1 \times 10^{33}$ & $90 \times 10^{-3}$ & 1.1 \\
\hline$p$ & ${ }^{3} \mathrm{He}^{\uparrow}$ & $1.5 \times 10^{17}$ & $1.0 \times 10^{15}$ & $3.7 \times 10^{33}$ & $12 \times 10^{-2}$ & 4.4 \\
\hline$p$ & $\mathrm{H}_{2}$ & $2.8 \times 10^{17}$ & $1.6 \times 10^{15}$ & $5.8 \times 10^{33}$ & $48 \times 10^{-3}$ & 3.1 \\
\hline$p$ & $\{\mathrm{Ne}, \mathrm{Ar}, \mathrm{Kr}, \mathrm{Xe}\}$ & $1.3 \times 10^{15}$ & $6.4 \times 10^{13}$ & $2.3 \times 10^{32}$ & $\{0.6,1.0,1.6,2.2\}$ & $\{1.6,2.6,4.2,5.7\}$ \\
\hline $\mathrm{Pb}$ & $\mathrm{H}^{\uparrow}$ & $6.5 \times 10^{16}$ & $2.5 \times 10^{14}$ & $1.2 \times 10^{29}$ & 3.0 & 15 \\
\hline $\mathrm{Pb}$ & $\mathrm{D}^{\uparrow}$ & $3.4 \times 10^{16}$ & $2.2 \times 10^{14}$ & $8.8 \times 10^{28}$ & 4.0 & 15 \\
\hline $\mathrm{Pb}$ & ${ }^{3} \mathrm{He}^{\uparrow}$ & $2.7 \times 10^{16}$ & $1.8 \times 10^{14}$ & $8.3 \times 10^{28}$ & 4.3 & 15 \\
\hline $\mathrm{Pb}$ & $\mathrm{H}_{2}$ & $6.5 \times 10^{16}$ & $2.5 \times 10^{14}$ & $1.2 \times 10^{29}$ & 3 & 15 \\
\hline $\mathrm{Pb}$ & $\mathrm{Xe}$ & $1.3 \times 10^{15}$ & $6.4 \times 10^{13}$ & $3.0 \times 10^{28}$ & 12 & 15 \\
\hline
\end{tabular}

Table 5

A selection of beam type, target type, target density $(\rho)$, target molar mass $(M)$, target thickness $(\ell)$, target areal density $\left(\theta_{\text {target }}\right)$, useable particle flux in the halo $\phi$ useable halo and instantaneous luminosity $\left(\mathcal{L}=\phi\right.$ useable halo $\left.\theta_{\text {target }}\right)$ for internal wire targets positioned in the halo of the LHC beams. The target areal density $\theta_{\text {target }}$ is equal to $\mathcal{N}_{\mathcal{A}} \ell \rho / M$ with $\mathcal{N}_{\mathcal{A}}$ the Avogadro number in $\mathrm{mol}^{-1}$.

\begin{tabular}{llllll}
\hline Beam & Target & $\begin{array}{l}\rho \\
{\left[\mathrm{g} \times \mathrm{cm}^{-3}\right]}\end{array}$ & $\begin{array}{l}\text { }\left[\mathrm{g} \times \mathrm{mol}^{-1}\right] \\
{[\mu \mathrm{m}]}\end{array}$ & $\begin{array}{l}\theta_{\text {target }} \\
{\left[\mathrm{cm}^{-2}\right]}\end{array}$ \\
\hline$p$ & $\mathrm{C}$ & 2.25 & 12 & 500 & $5.6 \times 10^{21}$ \\
{$\left[\mathrm{~cm}^{-2} \times \mathrm{s}^{-1}\right]$}
\end{tabular}

\subsection{Internal solid target intercepting the beam halo}

Another possible internal target solution for the LHC is to use a wire, a ribbon or a foil positioned in the halo of the beam as first proposed in [106]. Such an approach was adopted by the HERA-B [107] experiment with the prerequisite of not affecting experiments functioning on the $920 \mathrm{GeV}$ proton beam. The HERA-B system consisted of 2 stations of 4 wires each, made of Ti and $\mathrm{W}$, positioned in a square shape around the beam. For $\mathrm{C}$, flat ribbons were used. ${ }^{10}$ These could be positioned independently and adjusted with respect to the beam condition in order to scrap the beam halo. Other materials were also considered such as $\mathrm{Al}$, Fe and $\mathrm{Cu}$. One of the main limitations for HERA-B was the widening of the beam due to multiple Coulomb scattering in the target, which is reduced with low $Z$ materials. Multiple Coulomb scattering is not a limitation anymore when the beam halo is used, due to the lower halo intensity. For the LHC, simulations and tests (as done for the bent crystals discussed in the next section) are therefore needed to completely assess the feasibility and then the performance of such a system.

In principle, such a target system can be placed in the vicinity (or even inside) ALICE or LHCb at a moderate cost. If the system was found to be incompatible with high intensity runs, it could be used only for heavy-ion runs or during special runs; this would in turn reduce the collected luminosity in $p A$ collisions. Another limiting factor is the impossibility to carry out $p p$ collisions which are extremely important to quantify the nuclear effects. In the absence of such reference, it is mandatory to have at one's disposal measurements made with species with sufficiently different $A$. However, there are strong constraints from the LHC on the species which can be placed inside the LHC beam pipe. Therefore the mechanics for the positioning of the target should allow one to shift the target during the tuning of the beam. We have considered so $\mathrm{far}^{11} \mathrm{C}$, Ti, and $\mathrm{W}$ as possible species but further studies might be required (also for other species). Finally, let us note that such a system does not allow one to use a polarised target. The solid target parameters and the instantaneous luminosities obtained using typical LHC beam halo fluxes are shown in Table 5, for each type of beams and targets.

\footnotetext{
10 The diameter of the wires was $50 \mu \mathrm{m}$ whereas the ribbons were $100 \mu \mathrm{m}$ wide in the direction perpendicular to the beam and $500 \mu \mathrm{m}$ along the beam (which can thus be considered as the target thickness).

11 Note that the usage of a solid $\mathrm{Pb}$ target is a priori excluded by LHC experts due to the low melting temperature of the Pb.
} 


\subsection{External/internal target solution with a slow beam extraction using a bent crystal}

\subsubsection{Crystal-assisted extraction of the LHC beams}

The idea of a controlled non-resonant slow extraction of the CERN LHC beams - on the order of $10^{8}$ protons per second - to be used for fixed target physics is not new. Already, in the early 90's, the LHB collaboration submitted a letter of intent to the LHCC to get an experiment based on bent-crystal extraction approved. At the time, it was not clear whether such a technique could be used in the LHC conditions.

Since then, significant progresses were achieved with successful tests for protons at the SPS [130], Fermilab [131], Protvino [132] and for Pb ions at the SPS [133]. These were made possible by numerous experimental advances, like the improvement of the crystal quality with a production technique allowing to reach a channelling efficiency close to the theoretical one, or like the development of goniometers matching the critical angle requirements for a $6.5 \mathrm{TeV}$ beam channelling. Thanks to this legacy, the UA9 collaboration proposed this technique as a smart alternative for the upgrade of the LHC collimation system $[134,135]$ following the concept developed in the frame of the INTAS programme 03-51-6155, see for instance Ref. [136] issued in 2002. Tests were recently successfully carried out at the betatron cleaning insertion (IR7) both at injection and top energy (6.5 TeV) in 2015 [104]. Tests have also been performed with lead beam at injection and top energies $(2.5 \mathrm{TeV})$ at the end of 2016. They clearly demonstrated the feasibility of crystal-assisted collimation and, in turn, gave a new momentum in the plans for crystal-assisted extraction (see also [137]). Let us also stress that the crystal degradation due to radiation, once thought to be an issue, is negligible as demonstrated by tests [138] with the HiRadMat facility of the SPS.

The generic requirements for such an extraction system were already outlined in 1990 at the Aachen LHC workshop where it was identified that [139]:

- an extraction outside the ring is preferred to avoid interferences with the main tunnel and the experimental cavern;

- an extraction in the horizontal plane would probably be more favourable since an enhanced deflection ${ }^{12}$ could be achieved by an appropriate re-design of the separation recombination dipoles D1 and D2;

- the extraction would be at one of the odd points of the LHC;

- the crystal location would be between the quadrupoles Q3 and Q4 and it would provide a deflection of about 1 mrad;

- a further deflection up to 20 mrad could be achieved at $250 \mathrm{~m}$;

- increasing the size of the halo would result in a higher extraction efficiency with more particles crossing the crystal.

To be more quantitative about the required modifications of the beam pipe, since the halo is located at approximately $3 \mathrm{~mm}$ from the beam pipe axis, a deflection by an angle of $1 \mathrm{mrad}$ would result in these particles exiting the beam pipe at $30 \mathrm{~m}$ downstream, considering a LHC beam pipe radius of 3-4 cm in the LHC tunnel. Another proposal was made in 2005 [140] consisting in "replacing" the kicker-modules in LHC section IR6 (the beam dump) by a bent crystal that would provide the particles in the beam halo with a sufficient kick to overcome the septum blade and to be extracted. It is however not clear to which extent the beam-dump area, even with obvious modifications to move the beam-dump facility, can be used for experiments. As for now, beyond the generic requirements above, the possible locations of a possible extraction zone have not been listed. Currently, there is an active project, CRYSBEAM [141,142], whose objective is to demonstrate the feasibility of crystal-assisted extraction on the LHC and which should upon its completion give us better insight on the technical realisation of this solution. Operating the crystal in real parasitic mode still requires further studies.

Another possibility, which would avoid further manipulating the beam downstream of the crystal, is to directly use the extracted - and highly collimated - particles of the halo (which we refer to as the split beam) on an (semi) internal target system which would however not interact with the main LHC beam. Such a solution would probably allow one to limit the civil-engineering work to a minimum and also to use an existing experiment. Two caveats have however to be addressed. First, only a fraction of the split beam would interact with the target and the remaining $10^{8}$ protons per second should be absorbed or deviated from the experiment. Second, this would probably induce a non-negligible azimuthal asymmetry in the experimental system. For spin-related analyses, this may be a serious limitation in the cancellation of some systematical uncertainties. A first set-up (see Appendix A.2), compatible with the LHCb detector, has been proposed to measure the electric and magnetic dipole moments of charmed charged baryons at LHC top energies [143-145]. A first bent crystal, located at $5 \sigma$ from the centre beam line, deflects the halo particles by about $150 \mu$ rad at about $100 \mathrm{~m}$ upstream of the LHCb interaction region, in order to separate them from the circulating beam. A target, $1 \mathrm{~cm}$ long and $5 \mathrm{~mm}$ thick along the beam direction, inserted in the pipe intercepts the halo, producing short-lived baryons. A second crystal $^{13}$ channels part of the baryons and deflects them by 7 mrad $^{14}$ into the LHCb detector, where the spin orientation

\footnotetext{
12 The enhanced deflection would be beyond the one resulting from the crystal pass.

13 Note that this second crystal is needed in the case of electric or magnetic dipole moment measurements but not for the physics cases developed in that paper.

14 Let us remind that the deflection angle is related to the critical radius of the bent crystal, which increases linearly with the energy. At high energy, to reach larger angles, the length of the crystal has to be increased.
} 
Table 6

A selection of beam type, target type, target density $(\rho)$, target molar mass $(M)$, target thickness $(\ell)$, target areal density $\left(\theta_{\text {target }}\right)$, useable particle

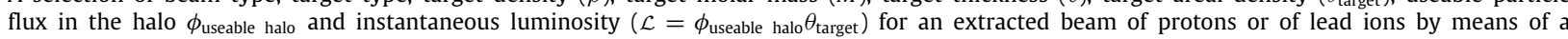
bent crystal and impinging a solid target. The target areal density is computed as in Table 5 . With a beam splitting option, there is currently no clear solution to allow for the usage of a light target (solid/liquid $\mathrm{H}, \mathrm{D}$ ).

\begin{tabular}{|c|c|c|c|c|c|c|c|}
\hline Beam & Target & $\begin{array}{l}\rho \\
{\left[\mathrm{g} \times \mathrm{cm}^{-3}\right]}\end{array}$ & $\begin{array}{l}M \\
{\left[\mathrm{~g} \times \mathrm{mol}^{-1}\right]}\end{array}$ & $\begin{array}{l}\ell \\
{[\mathrm{mm}]}\end{array}$ & $\begin{array}{l}\theta_{\text {target }} \\
{\left[\mathrm{cm}^{-2}\right]}\end{array}$ & $\begin{array}{l}\phi_{\text {useable halo }} \\
{\left[\mathrm{s}^{-1}\right]}\end{array}$ & $\begin{array}{l}\mathcal{L} \\
{\left[\mathrm{cm}^{-2} \mathrm{~s}^{-1}\right]}\end{array}$ \\
\hline$p$ & solid $\mathrm{H}$ & $8.80 \times 10^{-2}$ & 1 & 5 & $2.6 \times 10^{22}$ & $5.0 \times 10^{8}$ & $1.3 \times 10^{31}$ \\
\hline$p$ & $\mathrm{C}$ & 2.25 & 12 & 5 & $5.6 \times 10^{22}$ & $5.0 \times 10^{8}$ & $2.8 \times 10^{31}$ \\
\hline$p$ & $\mathrm{Ti}$ & 4.43 & 48 & 5 & $2.8 \times 10^{22}$ & $5.0 \times 10^{8}$ & $1.4 \times 10^{31}$ \\
\hline$p$ & $\mathrm{~W}$ & 19.3 & 184 & 5 & $3.1 \times 10^{22}$ & $5.0 \times 10^{8}$ & $1.6 \times 10^{31}$ \\
\hline $\mathrm{Pb}$ & solid $\mathrm{H}$ & $8.80 \times 10^{-2}$ & 1 & 5 & $2.6 \times 10^{22}$ & $1.0 \times 10^{5}$ & $2.6 \times 10^{27}$ \\
\hline $\mathrm{Pb}$ & $\mathrm{C}$ & 2.25 & 12 & 5 & $5.6 \times 10^{22}$ & $1.0 \times 10^{5}$ & $5.6 \times 10^{27}$ \\
\hline $\mathrm{Pb}$ & $\mathrm{Ti}$ & 4.43 & 48 & 5 & $2.8 \times 10^{22}$ & $1.0 \times 10^{5}$ & $2.8 \times 10^{27}$ \\
\hline $\mathrm{Pb}$ & $\mathrm{W}$ & 19.3 & 184 & 5 & $3.1 \times 10^{22}$ & $1.0 \times 10^{5}$ & $3.1 \times 10^{27}$ \\
\hline
\end{tabular}

is measured. An additional absorber intercepts the halo particles non-interacting with the target, thereby allowing the possibility of fixed-target operation in parasitic mode. An initial test of the beam-splitting concept and of the double crystal use in an accelerator was made in the SPS by the UA9 collaboration in 2017 [105]. However, there is still a long way before achieving a fully effective scenario, compatible with the LHC-collimation system and with the LHCb detector.

\subsubsection{Unpolarised targets}

As aforementioned, the LHC beams can be extracted by means of a bent crystal with typical fluxes on the order of $5 \times 10^{8} \mathrm{~s}^{-1}$ for the proton beam ${ }^{15}$ and $10^{5} \mathrm{~s}^{-1}$ for the lead beam. We will consider $5 \mathrm{~mm}$-thick targets, which are in principle compatible with both the split beam option and a dedicated beam line. Table 6 displays the corresponding instantaneous luminosities with the same species as for the internal wire target as well as for solid hydrogen to illustrate the case of light elements.

We stress that for an experiment with a specific target system away from the beampipe, targets as thick as a metre can be used for light species like H or D. Thicker targets can also be used for heavier species provided that the effect of multiple scatterings in the target can be mitigated. In general, the targets being thicker than in the internal-wire-target case ( 5 vs. $0.5 \mathrm{~mm}$ ), instantaneous luminosities are larger.

\subsubsection{Polarised targets}

One of the main thrusts of the proposed physics with AFTER@LHC will be the measurement of the Sivers asymmetry which will require a transversely polarised target. In the following, we present two possible target systems for AFTER@LHC, one inspired from the E1039 project at Fermilab [77] and the other inspired by the polarised target of the COMPASS experiment at CERN [147-149]. In both cases, the polarisation of the target relies on the Dynamic Nuclear Polarisation (DNP) method, whose general principle is described in [150]. Both targets could be envisioned in the case of crystalassisted extraction of the LHC beams into a new cavern, however, if redesigned, the E1039 target might also be useable with the crystal beam splitting solution.

The E1039 target. This target consists of a split coil superconducting magnet, operating at $5 \mathrm{~T}$. The coils are arranged such that the B field is either parallel or anti-parallel to the vertical direction, resulting in a transverse polarisation. Inside the magnet there is a refrigerator, which provides the necessary cooling power to keep the target at $1 \mathrm{~K}$. In the centre of the whole system resides the target stick, which contains the target cells, the microwave horn and the Nuclear-MagneticResonance (NMR) coils to measure the actual polarisation. The target insert has 2 active cells filled with frozen $\mathrm{NH}_{3}$ beads, one empty and one with a carbon target. The cells have an elliptical cross section, with one half axis being $1.9 \mathrm{~cm}$ diameter and the other one $2.1 \mathrm{~cm}$ while the length is $8 \mathrm{~cm}$. The target material is positioned in a liquid He bath, cooled to $1 \mathrm{~K}$, by lowering the vapour pressure of liquid He to 0.117 Torr. This is achieved with a dedicated system which have a capacity of pumping $15000 \mathrm{~m}^{3} / \mathrm{h}$ He gas. The microwave horn is sitting above the two top cells, which contain $\mathrm{NH}_{3}$ and $\mathrm{ND}_{3}$, thus allowing to measure polarised $p$ and $n$ under identical run and target conditions. This greatly reduces the systematic uncertainties by comparing $p$ and $n$. While both cells see the radiation from the microwave, only the one in the centre region of the coils will be polarised. Since the material has to be uniformly cooled, the ammonia is in the form of small frozen beads, which reduces the maximum density by a packing fraction of about 0.6 . From the planned beam intensities, we estimate that the material has to be changed every 140 days, due to radiation damage. In Fig. 55 of Appendix A.3, a schematic view of the E1039 target is shown.

\footnotetext{
15 Recent LHC collimation studies show that, for parasitic operation, the layout proposed for LHCb could deliver at least $10^{6}$ protons per second [146]. Another scenario with controlled excitation of beam losses for selected proton bunches would provide higher flux but would need further studies.
} 
Table 7

Target type, target density $(\rho)$, target thickness $(\ell)$, target molar mass $(M)$, effective target areal density ( $\theta_{\text {target }}^{\text {(eff), }}$, useable particle flux in the halo

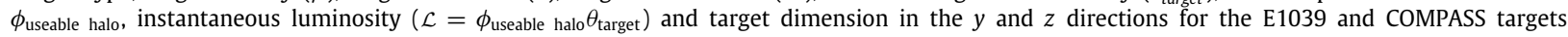
As what regards the target geometry, we note that the E1039 target is cylindrical. As in Table 5 , the target areal density is defined as $\theta_{\text {target }}=$ $\left(\mathcal{N}_{\mathcal{A}} \ell \rho\right) / M$. However, in order to account for the fact that the target material does not occupy all the volume of the cell due to technicalities during filling of the cell and the presence of small amounts of coolant (He), additional factors need to be considered. The cell density is expressed as $\rho_{\text {cell }}=\rho_{\mathrm{NH}_{3}} P F+\rho_{\mathrm{He}}(1-P F)$, where $P F$ is the packing factor (we considered $P F=0.6$ for the $\mathrm{NH}_{3}, \mathrm{ND}_{3}$ and butanol targets, and 0.55 for ${ }^{6} \mathrm{LiD}$ ). The second term, $\rho_{\mathrm{He}}(1-P F)$, however is neglected in our calculation. It is called the rest space factor and amounts to about $10 \%$. We thus introduced the effective target areal density such that $\theta_{\text {target }}^{\text {(eff.) }}=\theta_{\text {target }} P F$.

\begin{tabular}{|c|c|c|c|c|c|c|c|c|}
\hline & Target & $\begin{array}{l}\rho \\
{\left[\mathrm{g} \mathrm{cm}^{-3}\right]}\end{array}$ & $\begin{array}{l}\ell \\
{[\mathrm{cm}]}\end{array}$ & $\begin{array}{l}M \\
{[\mathrm{~g} \mathrm{~mol}-1]}\end{array}$ & $\begin{array}{c}\theta_{\text {target }}^{\text {(eff. }} \\
{\left[\mathrm{cm}^{-2}\right]}\end{array}$ & $\begin{array}{l}\phi_{\text {useable halo }} \\
{\left[\mathrm{s}^{-1}\right]}\end{array}$ & $\stackrel{\mathcal{L}}{\left[\mathrm{cm}^{-2} \mathrm{~s}^{-1}\right]}$ & $\begin{array}{c}y \times z \\
{[\mathrm{~mm} \times \mathrm{mm}]}\end{array}$ \\
\hline \multirow{2}{*}{ E1039 target } & $\mathrm{NH}_{3}^{\uparrow}$ & 0.86 & 8 & 17 & $1.4 \times 10^{23}$ & $5.0 \times 10^{8}$ & $7.2 \times 10^{31}$ & \multirow{2}{*}{$1853 \times 975$} \\
\hline & $\mathrm{ND}_{3}^{\uparrow}$ & 1.01 & 8 & 20 & $1.4 \times 10^{23}$ & $5.0 \times 10^{8}$ & $7.2 \times 10^{31}$ & \\
\hline \multirow{3}{*}{ COMPASS target } & $\mathrm{NH}_{3}^{\uparrow}$ & 0.86 & 110 & 17 & $2.0 \times 10^{24}$ & $5.0 \times 10^{8}$ & $1.0 \times 10^{33}$ & \multirow{3}{*}{$2820 \times 3120$} \\
\hline & ${ }^{6} \mathrm{LiD}^{\uparrow}$ & 0.84 & 110 & 8 & $3.9 \times 10^{24}$ & $5.0 \times 10^{8}$ & $1.9 \times 10^{33}$ & \\
\hline & butanol $^{\uparrow}$ & 0.99 & 110 & 74 & $5.3 \times 10^{23}$ & $5.0 \times 10^{8}$ & $2.7 \times 10^{32}$ & \\
\hline
\end{tabular}

The COMPASS target. The target consists of two identical, $55 \mathrm{~cm}$ long cylindrical cells with a diameter of $4 \mathrm{~cm}$. Each cell has 5 NMR coils to measure the polarisations [151] (see Fig. 57 of Appendix A.4 for a schematic view of the COMPASS target). The cells can be polarised in opposite direction and there is a $20 \mathrm{~cm}$ long gap between the cells, in order to cleanly separate interactions from the respective target cells. The orientations are reversed by changing microwave frequency at $2.5 \mathrm{~T}$ at regular intervals in order to reduce the systematic error. The polarisation is obtained by the DNP method with a high cooling power dilution refrigerator with a $13500 \mathrm{~m}^{3} \mathrm{~h}^{-1}$ pumping speed of 8 Pfeiffer Roots blowers in series, a $2.5 \mathrm{~T}$ solenoid magnet and two microwave systems of about $70 \mathrm{GHz}$ [149]. The spin can be oriented perpendicular to the beam direction by using a $0.6 \mathrm{~T}$ dipole magnet. Under this magnetic condition the polarisation cannot be enhanced by the DNP method, but can be maintained at a lattice temperature below $100 \mathrm{mK}$. The proton polarisation achieved in 2015 with $\mathrm{NH}_{3}$ was $80 \%$ in 1 day and about $90 \%$ after 2 days. ${ }^{16}$ In a beam intensity of $8 \times 10^{7}$ pions $\mathrm{s}^{-1}$ the beam intensity for each $\mathrm{NH}_{3}$ bead of $2-3 \mathrm{~mm}$ is about $10^{6} \mathrm{~s}^{-1}$ which will not lead to a significant depolarisation in the frozen spin mode. No significant radiation damage could be observed in more than half a year of data taking in 2015 at COMPASS. If needed for the case of $5 \times 10^{8} \mathrm{p} / \mathrm{s}$ beam intensity with a more focused beam, the target material may be considered to be annealed twice or thrice a year ${ }^{17}$ to recover the optimal target performances. A spin relaxation time of about $1000 \mathrm{~h}$ was measured at $0.6 \mathrm{~T}$ and $50 \mathrm{mK}$ for the proton in $\mathrm{NH}_{3}$.

Comparison. Table 7 displays the parameters of the E1039 target and of the COMPASS target, which could be used for AFTER@LHC. The COMPASS target permits to reach higher luminosities than the E1039 target, however the E1039 target offers several advantages. The latter is smaller and could therefore be used in beam splitting mode if a significant modification of the beam line and target is performed. Both also permit the usage of polarised deuterium target $\left(\mathrm{ND}_{3}\right.$ or $\left.{ }^{6} \mathrm{LiD}\right)$, complementary to the hydrogen one $\left(\right.$ e.g. $\left.\mathrm{NH}_{3}\right)$.

\subsection{Comparison of technologies}

In this section, we will discuss a qualitative comparison of the various technological solutions which have been developed. More quantitative comparisons of the instantaneous luminosities which could be achieved and performances for STSA measurements for the various solutions will also be presented.

\subsubsection{Qualitative comparison of the various technological solutions}

Table 8 gathers our qualitative judgement of the different solutions with regards to a number of decisional criteria and to the reach in the three physics cases developed in the Sections 5.1, 5.2 and 5.3 .

In particular, we stress that it is assumed here that one uses a detector without specific data-taking-rate limitations. The physics reach when using the ALICE and LHCb detectors will be discussed in Section 4.

For the internal-gas-target solutions, the current SMOG system in LHCb has the advantage to be mostly parasitic to other LHC experiments due to its low gas density. Also, various noble gases up to argon have already been used. However, its duration time is limited as well as the possible yearly integrated luminosity and it cannot run with polarised gas. Furthermore, the luminosity can barely be directly estimated.

To achieve the physics reach proposed in this report with gas-target solutions, it is important to increase the gas density with respect to SMOG and to opt for a more flexible gas system with polarised gases for spin physics. Moreover, running

\footnotetext{
16 The proton polarisation for the $\mathrm{NH}_{3}$ target and for the 2015 analysed data sample was in average 73\% [81].

17 The process of the annealing consists of removing the ${ }^{3} \mathrm{He}$ gas, warming up to $70 \mathrm{~K}$, cooling down and re-filling with ${ }^{3} \mathrm{He}$. In total it takes one week for the COMPASS system.
} 
Table 8

Qualitative comparison of the various technological solutions.

\begin{tabular}{|c|c|c|c|c|c|c|}
\hline \multirow[b]{2}{*}{ Characteristics } & \multicolumn{3}{|c|}{ Internal-gas target } & \multirow[t]{2}{*}{ Internal-solid target with beam halo } & \multirow[t]{2}{*}{ Beam splitting } & \multirow[t]{2}{*}{ Beam extraction } \\
\hline & SMOG & Gas Jet & Storage Cell & & & \\
\hline Run duration $^{\mathrm{a}}$ & $\star$ & $\star \star$ & $\star \star$ & $\star$ & $\star \star$ & $\star \star \star$ \\
\hline Parasiticity $^{\mathrm{b}}$ & $\star \star$ & $\star \star$ & $\star \star$ & $\star$ & $\star \star$ & $\star \star \star$ \\
\hline Integrated luminosity ${ }^{c}$ & $\star$ & $\star \star \star$ & $\star \star \star$ & $\star$ & $\star \star$ & $\star \star \star$ \\
\hline Absolute luminosity determination ${ }^{\mathrm{d}}$ & $\star$ & $\star \star$ & $\star \star$ & $\star$ & $\star \star$ & $\star \star \star$ \\
\hline Target versatility $^{\mathrm{e}}$ & $\star$ & $\star \star$ & $\star \star$ & $\star$ & $\star \star$ & $\star \star \star$ \\
\hline (Effective) target polarisation ${ }^{\mathrm{f}}$ & - & $\star \star \star$ & $\star \star$ & - & $-1 \star^{g}$ & $\star$ \\
\hline Use of existing experiment ${ }^{\mathrm{h}}$ & $\star \star \star$ & $\star \star$ & $\star$ & $\star \star$ & $\star \star$ & - \\
\hline Civil engineering or $R \& D{ }^{i}$ & $\star \star \star \star$ & $\star \star \star$ & $\star \star$ & $\star \star$ & $\star \star$ & $\star$ \\
\hline Cost & $\star \star \star$ & $\star \star$ & $\star \star$ & $\star \star \star$ & $\star \star$ & $\star$ \\
\hline Implementation time & $\star \star \star$ & $\star \star$ & $\star \star$ & $\star \star \star$ & $\star \star$ & $\star$ \\
\hline High $x$ & $\star$ & $\star \star \star$ & $\star \star \star \star$ & $\star$ & $\star \star$ & $\star \star \star \star$ \\
\hline Spin Physics & - & $\star \star \star$ & $\star \star \star$ & - & $-\mid \star \star$ & $\star \star \star$ \\
\hline Heavy-Ion & $\star$ & $\star \star \star$ & $\star \star \star$ & $\star \star$ & $\star \star$ & $\star \star \star \star$ \\
\hline
\end{tabular}

a $\star \star \star$ : no limitation; $\star \star:$ possible limitation; $\star$ : data taking for special runs only.

$\mathrm{b} \star \star \star$ : no impact on the other LHC experiments; $\star \star$ : no impact pending constraints; $\star$ : significant impact.

$c_{\star} \star \star$ : highest; $\star \star$ : high; $\star$ : moderate [corresponding to 1 LHC year].

$\mathrm{d}_{\star \star \star}$ : direct with small uncertainty; $\star \star$ : direct with moderate uncertainty; $\star$ : indirect.

${ }^{\mathrm{e}} \star \star \star$ : no limitation; $\star \star$ : some target types are not possible; $\star$ : only a few target types are possible.

$\mathrm{f}_{\star \star \star} \star$ : highest; $\star \star$ : high; $\star$ : moderate.

g With a redesigned E1096 target.

$\mathrm{h}_{\star \star \star} \star$ : without any experiment/beam-pipe modifications; $\star \star$ : with slight experiment/beam-pipe modifications; $\star$ : with slight experiment/beam-pipe modifications and potential non-optimal acceptances.

${ }^{\mathrm{i}} \star \star \star \star$ : none; $\star \star \star$ : some R\&D; $\star \star$ : some R\&D with possible show stoppers; $\star$ significant civil engineering.

with hydrogen gas allows one to obtain a reference measurement with protons as target for high- $x$ and heavy-ion physics. For that purpose, the gas-jet and storage-cell solutions are probably the most promising - with the highest luminosity for the storage-cell solution. Note, however, that the cell coating has to be compatible with the LHC vacuum constraints, which seems not to be the case of the original HERMES target. In the target chamber of the gas-jet system, one can also inject nuclear-target gases, however this was not tested at RHIC and the possible gas density in that case was estimated.

The internal-solid-target solution directly on the beam halo has the advantage to be compatible with various target species. This solution suffers however from a low luminosity and will likely impact the LHC-beam stability. The beamsplitting solution, by using a slow beam extraction with a bent crystal, will probably have less impact on the LHC beam and will allow one to run for a longer period and with thicker targets. If coupled with a redesigned E1039 target, it would allow for spin physics.

Finally the beam-extraction solution is more suitable for the physics reach. However the necessary civil engineering would largely increase the cost by more than one order of magnitude and the implementation time with respect to the other solutions that are at reach with limited technical developments.

\subsubsection{Comparison of the luminosities achieved for AFTER@LHC with the various technological solutions}

Table 9 compares the instantaneous luminosities, the expected running time with the proton or lead beam and the integrated luminosities achievable in one LHC year of data taking, for the various technical solutions described in this section. These numbers should be interpreted as maxima, and can be decreased according to data-taking-detector capabilities (see Section 4). For the internal-solid target with beam-halo, beam-splitting and beam-extraction solutions, the fluxes of proton and lead on target are assumed to be $5 \times 10^{8} \mathrm{~s}^{-1}$ and $10^{5} \mathrm{~s}^{-1}$, respectively. As can be seen from Table 9, the highest luminosity which can be achieved in $\mathrm{pH}^{\uparrow}$ collisions is about $10 \mathrm{fb}^{-1}$ with a storage-cell gas target. Integrated luminosities of about the same order of magnitude could be reached with the E1039 target with a beamsplitting or beam-extraction option. The gas-jet solution gives luminosity about two orders of magnitude smaller than the storage cell for polarised hydrogen. For unpolarised $\mathrm{pH}_{2}$ collisions, the performances of the storage-cell and gas-jet targets are similar, of the order of $40-50 \mathrm{fb}^{-1}$. In proton-nucleus collisions for large nuclei, the storage-cell and gas-jet targets also give the best integrated luminosities (on the order of the $\mathrm{fb}^{-1}$ ). In order to obtain similar luminosities in $p \mathrm{~W}$ collisions with an internal solid target would require the width of the target to be of about $50 \mathrm{~mm}$ (or the usage of serial targets). In lead-nucleus collisions, the performances of the storage-cell and gas-jet targets are also the best, allowing for the collection of approximately $1 \mathrm{fb}^{-1}$ in $\mathrm{PbH}_{2}$ and $30 \mathrm{nb}^{-1}$ in PbXe collisions.

\subsubsection{Comparison of the polarised-target performances for STSA measurements}

Table 10 shows the comparison of the figure of merit for STSA measurements, for the various polarised targets described in this section. The nominal LHC-proton-beam flux is considered for the gaseous targets while the expected proton-beam flux extracted by means of a bent crystal is considered for the E1039 and COMPASS targets. While the 
Table 9

Summary table of the achievable integrated luminosities for the various technical solutions described in this section.

\begin{tabular}{|c|c|c|c|c|c|c|c|c|}
\hline \multirow[t]{3}{*}{ Target } & & & \multicolumn{6}{|l|}{ Beam } \\
\hline & & & \multicolumn{3}{|l|}{$p$} & \multicolumn{3}{|l|}{$\mathrm{Pb}$} \\
\hline & & & $\begin{array}{l}\mathcal{L} \\
{\left[\mathrm{cm}^{-2} \mathrm{~s}^{-1}\right]}\end{array}$ & $\begin{array}{l}\Delta t \\
{[\mathrm{~s}]}\end{array}$ & $\int \mathcal{L}$ & $\begin{array}{l}\mathcal{L} \\
{\left[\mathrm{cm}^{-2} \mathrm{~s}^{-1}\right]}\end{array}$ & $\begin{array}{l}\Delta t \\
{[\mathrm{~s}]}\end{array}$ & $\int \underset{\left[\mathrm{nb}^{-1}\right]}{\mathcal{L}}$ \\
\hline \multirow{3}{*}{$\begin{array}{l}\text { Internal gas } \\
\text { target }\end{array}$} & SMOG & $\mathrm{He}, \mathrm{Ne}, \mathrm{Ar}$ & $5.8 \times 10^{29}$ & $2.5 \times 10^{5}$ & $1.5 \times 10^{2}$ & $7.4 \times 10^{25}$ & $1.0 \times 10^{6}$ & $7.4 \times 10^{-2}$ \\
\hline & Gas-Jet & $\begin{array}{l}\mathrm{H}^{\uparrow} \\
\mathrm{H}_{2} \\
\mathrm{D}^{\uparrow} \\
{ }^{3} \mathrm{He}^{\uparrow} \\
\mathrm{Xe}\end{array}$ & $\begin{array}{l}4.3 \times 10^{30} \\
3.6 \times 10^{33 \div 34} \\
4.3 \times 10^{30} \\
3.6 \times 10^{32} \\
(3.6 \div 18) \times 10^{31}\end{array}$ & $\begin{array}{l}1.0 \times 10^{7} \\
1.0 \times 10^{7} \\
1.0 \times 10^{7} \\
1.0 \times 10^{7} \\
1.0 \times 10^{7}\end{array}$ & $\begin{array}{l}4.3 \times 10^{4} \\
3.6 \times 10^{7 \div 8} \\
4.3 \times 10^{4} \\
3.6 \times 10^{6} \\
(3.6 \div 18) \times 10^{5}\end{array}$ & $\begin{array}{l}5.6 \times 10^{26} \\
1.2 \times 10^{29} \\
5.6 \times 10^{26} \\
4.7 \times 10^{28} \\
(0.5 \div 2.3) \times 10^{28}\end{array}$ & $\begin{array}{l}1.0 \times 10^{6} \\
1.0 \times 10^{6} \\
1.0 \times 10^{6} \\
1.0 \times 10^{6} \\
1.0 \times 10^{6}\end{array}$ & $\begin{array}{l}5.6 \times 10^{-1} \\
1.2 \times 10^{2} \\
5.6 \times 10^{-1} \\
47 \\
5.0 \div 23\end{array}$ \\
\hline & Storage Cell & $\begin{array}{l}\mathrm{H}^{\uparrow} \\
\mathrm{H}_{2} \\
\mathrm{D}^{\uparrow} \\
{ }^{3} \mathrm{He}^{\uparrow} \\
\mathrm{Xe}\end{array}$ & $\begin{array}{l}9.2 \times 10^{32} \\
5.8 \times 10^{33} \\
1.1 \times 10^{33} \\
3.7 \times 10^{33} \\
2.3 \times 10^{32}\end{array}$ & $\begin{array}{l}1.0 \times 10^{7} \\
1.0 \times 10^{7} \\
1.0 \times 10^{7} \\
1.0 \times 10^{7} \\
1.0 \times 10^{7}\end{array}$ & $\begin{array}{l}9.2 \times 10^{6} \\
5.8 \times 10^{7} \\
1.1 \times 10^{7} \\
3.7 \times 10^{7} \\
2.3 \times 10^{6}\end{array}$ & $\begin{array}{l}1.2 \times 10^{29} \\
1.2 \times 10^{29} \\
8.8 \times 10^{28} \\
8.3 \times 10^{28} \\
3.0 \times 10^{28}\end{array}$ & $\begin{array}{l}1.0 \times 10^{6} \\
1.0 \times 10^{6} \\
1.0 \times 10^{6} \\
1.0 \times 10^{6} \\
1.0 \times 10^{6}\end{array}$ & $\begin{array}{l}1.2 \times 10^{2} \\
1.2 \times 10^{2} \\
88 \\
83 \\
30\end{array}$ \\
\hline $\begin{array}{l}\text { Target on the } \\
\text { beam halo }\end{array}$ & $\begin{array}{l}\text { Wire target } \\
(0.5 \mathrm{~mm})\end{array}$ & $\begin{array}{l}\mathrm{C} \\
\mathrm{Ti} \\
\mathrm{W}\end{array}$ & $\begin{array}{l}2.8 \times 10^{30} \\
1.4 \times 10^{30} \\
1.6 \times 10^{30}\end{array}$ & $\begin{array}{l}1.0 \times 10^{7} \\
1.0 \times 10^{7} \\
1.0 \times 10^{7}\end{array}$ & $\begin{array}{l}2.8 \times 10^{4} \\
1.4 \times 10^{4} \\
1.6 \times 10^{4}\end{array}$ & $\begin{array}{l}5.6 \times 10^{26} \\
2.8 \times 10^{26} \\
3.1 \times 10^{26}\end{array}$ & $\begin{array}{l}1.0 \times 10^{6} \\
1.0 \times 10^{6} \\
1.0 \times 10^{6}\end{array}$ & $\begin{array}{l}5.6 \times 10^{-1} \\
2.8 \times 10^{-1} \\
3.1 \times 10^{-1}\end{array}$ \\
\hline \multirow{2}{*}{ Beam splitting } & E1039 & $\begin{array}{l}\mathrm{NH}_{3}^{\uparrow} \\
\mathrm{ND}_{3}^{\uparrow}\end{array}$ & $\begin{array}{l}7.2 \times 10^{31} \\
7.2 \times 10^{31}\end{array}$ & $\begin{array}{l}1.0 \times 10^{7} \\
1.0 \times 10^{7}\end{array}$ & $\begin{array}{l}7.2 \times 10^{5} \\
7.2 \times 10^{5}\end{array}$ & $\begin{array}{l}1.4 \times 10^{28} \\
1.4 \times 10^{28}\end{array}$ & $\begin{array}{l}1.0 \times 10^{6} \\
1.0 \times 10^{6}\end{array}$ & $\begin{array}{l}14 \\
14\end{array}$ \\
\hline & $\begin{array}{l}\text { Unpolarised } \\
\text { solid } \\
\text { target }(5 \mathrm{~mm})\end{array}$ & $\begin{array}{l}\mathrm{C} \\
\mathrm{Ti} \\
\mathrm{W}\end{array}$ & $\begin{array}{l}2.8 \times 10^{31} \\
1.4 \times 10^{31} \\
1.6 \times 10^{31}\end{array}$ & $\begin{array}{l}1.0 \times 10^{7} \\
1.0 \times 10^{7} \\
1.0 \times 10^{7}\end{array}$ & $\begin{array}{l}2.8 \times 10^{5} \\
1.4 \times 10^{5} \\
1.6 \times 10^{5}\end{array}$ & $\begin{array}{l}5.6 \times 10^{27} \\
2.8 \times 10^{27} \\
3.1 \times 10^{27}\end{array}$ & $\begin{array}{l}1.0 \times 10^{6} \\
1.0 \times 10^{6} \\
1.0 \times 10^{6}\end{array}$ & $\begin{array}{l}5.6 \\
2.8 \\
3.1\end{array}$ \\
\hline \multirow{2}{*}{$\begin{array}{l}\text { Beam } \\
\text { extraction }\end{array}$} & E1039 & $\begin{array}{l}\mathrm{NH}_{3}^{\uparrow} \\
\mathrm{ND}_{3}^{\uparrow}\end{array}$ & $\begin{array}{l}7.2 \times 10^{31} \\
7.2 \times 10^{31}\end{array}$ & $\begin{array}{l}1.0 \times 10^{7} \\
1.0 \times 10^{7}\end{array}$ & $\begin{array}{l}7.2 \times 10^{5} \\
7.2 \times 10^{5}\end{array}$ & $\begin{array}{l}1.4 \times 10^{28} \\
1.4 \times 10^{28}\end{array}$ & $\begin{array}{l}1.0 \times 10^{6} \\
1.0 \times 10^{6}\end{array}$ & $\begin{array}{l}14 \\
14\end{array}$ \\
\hline & COMPASS & $\begin{array}{l}\mathrm{NH}_{3}^{\uparrow} \\
{ }^{6} \mathrm{LiD}^{\uparrow} \\
\text { butanol } \uparrow\end{array}$ & $\begin{array}{l}1.0 \times 10^{33} \\
1.9 \times 10^{33} \\
2.7 \times 10^{32}\end{array}$ & $\begin{array}{l}1.0 \times 10^{7} \\
1.0 \times 10^{7} \\
1.0 \times 10^{7}\end{array}$ & $\begin{array}{l}1.0 \times 10^{7} \\
1.9 \times 10^{7} \\
2.7 \times 10^{6}\end{array}$ & $\begin{array}{l}2.0 \times 10^{29} \\
3.9 \times 10^{29} \\
5.3 \times 10^{28}\end{array}$ & $\begin{array}{l}1.0 \times 10^{6} \\
1.0 \times 10^{6} \\
1.0 \times 10^{6}\end{array}$ & $\begin{array}{l}2.0 \times 10^{2} \\
3.9 \times 10^{2} \\
53\end{array}$ \\
\hline
\end{tabular}

\section{Table 10}

Comparison of the target performances for STSA measurements. From left to right: target, target polarisation $\left(P_{\mathrm{T}}\right)$, average dilution factor $(\langle f\rangle)$ or depolarisation factor $(\alpha)$, total number of nucleons in the target $\left(\sum_{i} A_{i}\right)$, target areal density $\left(\theta_{\text {target }}\right)$, instantaneous luminosity $(\mathcal{L})$, effective polarisation $\left(\mathcal{P}_{\text {eff }}\right)$ and spin figure of merit of the target and beam $(\mathcal{F})$.

\begin{tabular}{|c|c|c|c|c|c|c|c|}
\hline Target & $P_{T}$ & $\langle f\rangle$ or $\alpha$ & $\sum_{i} A_{i}$ & $\begin{array}{l}\theta_{\text {target }} \\
{\left[\mathrm{cm}^{-2}\right]}\end{array}$ & $\begin{array}{l}\mathcal{L} \\
{\left[\mathrm{cm}^{-2} \mathrm{~s}^{-1}\right]}\end{array}$ & $\mathcal{P}_{\text {eff }}^{2}$ & $\begin{array}{l}\mathcal{F} \\
{\left[\mathrm{cm}^{-2} \mathrm{~s}^{-1}\right]}\end{array}$ \\
\hline $\mathrm{NH}_{3}$ E1039 & 0.85 & 0.17 & 17 & $1.4 \times 10^{23}$ & $7.2 \times 10^{31}$ & 0.021 & $2.6 \times 10^{31}$ \\
\hline $\mathrm{ND}_{3}$ E1039 & 0.32 & 0.30 & 20 & $1.4 \times 10^{23}$ & $7.2 \times 10^{31}$ & 0.009 & $1.3 \times 10^{31}$ \\
\hline $\mathrm{NH}_{3}$ COMPASS & 0.90 & 0.18 & 17 & $2.0 \times 10^{24}$ & $1.0 \times 10^{33}$ & 0.025 & $4.3 \times 10^{32}$ \\
\hline Butanol COMPASS & 0.90 & 0.14 & 74 & $5.3 \times 10^{23}$ & $2.7 \times 10^{32}$ & 0.015 & $3.0 \times 10^{32}$ \\
\hline${ }^{6}$ LiD COMPASS & $\begin{array}{l}0.46^{\mathrm{a}} \\
0.43^{\mathrm{b}}\end{array}$ & 0.250 & 8 & $3.9 \times 10^{24}$ & $1.9 \times 10^{33}$ & 0.050 & $7.6 \times 10^{32}$ \\
\hline H HERMES like storage cell ${ }^{\mathrm{c}}$ & 0.85 & 0.95 & 1 & $2.5 \times 10^{14}$ & $9.2 \times 10^{32}$ & 0.650 & $6.0 \times 10^{32}$ \\
\hline${ }^{3} \mathrm{He}$ HERMES like storage cell ${ }^{\mathrm{d}}$ & 0.70 & 0.33 & 3 & $1.0 \times 10^{15}$ & $3.7 \times 10^{33}$ & 0.053 & $5.9 \times 10^{32}$ \\
\hline D HERMES like storage cell ${ }^{\mathrm{e}}$ & 0.85 & 0.92 & 2 & $2.9 \times 10^{14}$ & $1.1 \times 10^{33}$ & 0.610 & $1.3 \times 10^{33}$ \\
\hline H RHIC-like gas jet & 0.96 & 0.97 & 1 & $1.2 \times 10^{12}$ & $4.3 \times 10^{30}$ & 0.860 & $3.7 \times 10^{30}$ \\
\hline${ }^{3} \mathrm{He}$ RHIC-like gas jet & 0.70 & 0.33 & 3 & $1.0 \times 10^{14}$ & $3.6 \times 10^{32}$ & 0.053 & $5.8 \times 10^{31}$ \\
\hline D RHIC-like gas jet & 0.85 & $\mathcal{O}(1)$ & 2 & $1.2 \times 10^{12}$ & $4.3 \times 10^{30}$ & 0.720 & $6.2 \times 10^{30}$ \\
\hline
\end{tabular}

${ }^{\mathrm{a}}$ For D.

${ }^{\mathrm{b}}$ For ${ }^{6} \mathrm{Li}$.

${ }^{\mathrm{c}} T=300 \mathrm{~K}, \ell=100 \mathrm{~cm}$.

${ }^{\mathrm{d}} T=300 \mathrm{~K}, \ell=100 \mathrm{~cm}$.

${ }^{\mathrm{e}} T=300 \mathrm{~K}, \ell=100 \mathrm{~cm}$.

absolute systematic error on STSA measurements is governed by the precision on the luminosity measurement for the two polarisation states (strictly speaking, the determination of their relative luminosity), the relative error will mainly come from the knowledge of the polarisation (usually determined with a precision of about $3 \%-4 \%$, see e.g. [111,126]).

The 'HERMES' $\mathrm{H}$-gas target has the best figure of merit, on the order of $6 \times 10^{32} \mathrm{~cm}^{-2} \mathrm{~s}^{-1}$. Using a cooled storage cell at $T=100 \mathrm{~K}$, the gas density would increase by a factor of $\sqrt{3}$, leading to an increase in the instantaneous luminosity 


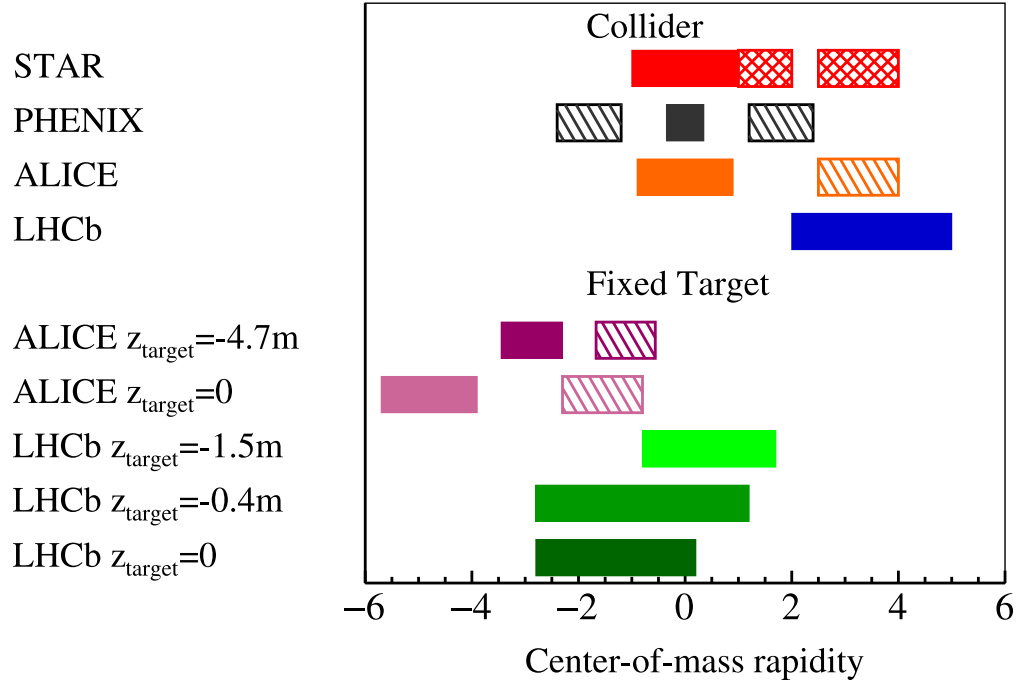

Fig. 8. Comparison of the kinematical coverages of the ALICE and LHCb detectors at the LHC and the STAR and PHENIX detectors at RHIC. For ALICE and LHCb, the acceptance is shown in the collider and the fixed-target modes for a $7 \mathrm{TeV}$ proton beam. For LHCb, the target position is at the nominal Interaction Point (IP), i.e. $z_{\text {target }}=0$, and the acceptance is also shown for two other positions corresponding to $z_{\text {target }}=-0.4 \mathrm{~m}$ and $-1.5 \mathrm{~m}$ on the opposite side of the spectrometer (see text for more details). For ALICE, the acceptances are shown for a target located at the IP as well as at $z_{\text {target }}=-4.7 \mathrm{~m}$ on the opposite side of the Muon spectrometer. The fully filled rectangles refer to detectors with particle identification capabilities, the double-hatched rectangles to electromagnetic calorimeters and the hatched rectangles to muon detectors.

to $1.59 \times 10^{33} \mathrm{~cm}^{-2} \mathrm{~s}^{-1}$, which is about $16 \%$ of the pp collider luminosity. The figure of merit $\mathcal{F}$ would increase up to $1.04 \times 10^{33} \mathrm{~cm}^{-2} \mathrm{~s}^{-1}$. The 'RHIC' H-jet-gas-target figure of merit is smaller by two orders of magnitudes with respect to the 'HERMES' H-gas target, because of the smaller achievable gas density. Similar performances as the 'RHIC' H-jet gas target can be reached with the solid E1039 and COMPASS targets. Due to the larger length of the COMPASS target, the figure of merit of the COMPASS target is better with respect to the E1039 one, at the cost of less portability and the likely impossibility to couple it to the beam-split option.

\section{Detector requirements and expected performances}

The ambitious physics case outlined in this document imposes significant requirements on the detector needed for such an experiment. The particle production is shifted towards larger angles, and the rapidity shifts are $\Delta y=4.2$ and 4.8 for a beam energy per nucleon of 2.76 and $7 \mathrm{TeV}$, respectively. Fig. 8 shows the rapidity acceptances of ALICE [152] and LHCb [153] detectors in the collider and fixed-target modes for a given target position (see caption), in comparison with the STAR [154-157] and PHENIX [158] detectors at RHIC.

As outlined by this comparison of LHC detectors used in a fixed-target mode, the major advantage of a fixed-target experiment is that particle production can be easily measured at very large values of negative- $y_{\text {c.m.s. }}$ with standard detector technologies. On the other hand, the full forward hemisphere is compressed into a very small solid angle area. The instantaneous luminosities with a fixed-target experiment by using the LHC beams are expected to be high, as described in the previous section, leading to large inelastic rates and allowing one to probe the full rapidity range with high statistics for many processes. In this section, we will first describe the general detector requirements in order to achieve the rich physics programme proposed in that paper for a fixed-target experiment at the LHC, and we will then discuss more specifically possible implementations with the existing detectors of the ALICE and LHCb experiments. The two implementations will be compared in terms of rapidity coverage, integrated luminosities and physics reach.

\subsection{Detector requirements}

The rapidity range in the laboratory frame of a fixed-target experiment should be as broad as possible covering the regions of backward and mid-rapidity in the c.m.s., i.e. from $y_{\text {lab. }}=0$ to $y_{\text {lab. }}=4.2$ and 4.8 with a beam energy per nucleon of 2.76 and $7 \mathrm{TeV}$, respectively. A multi-purpose experiment with detectors able to identify particles such as electrons, hadrons, photons as well as muons down to low $p_{T}$ would fit better the rich physics programme proposed here. A 

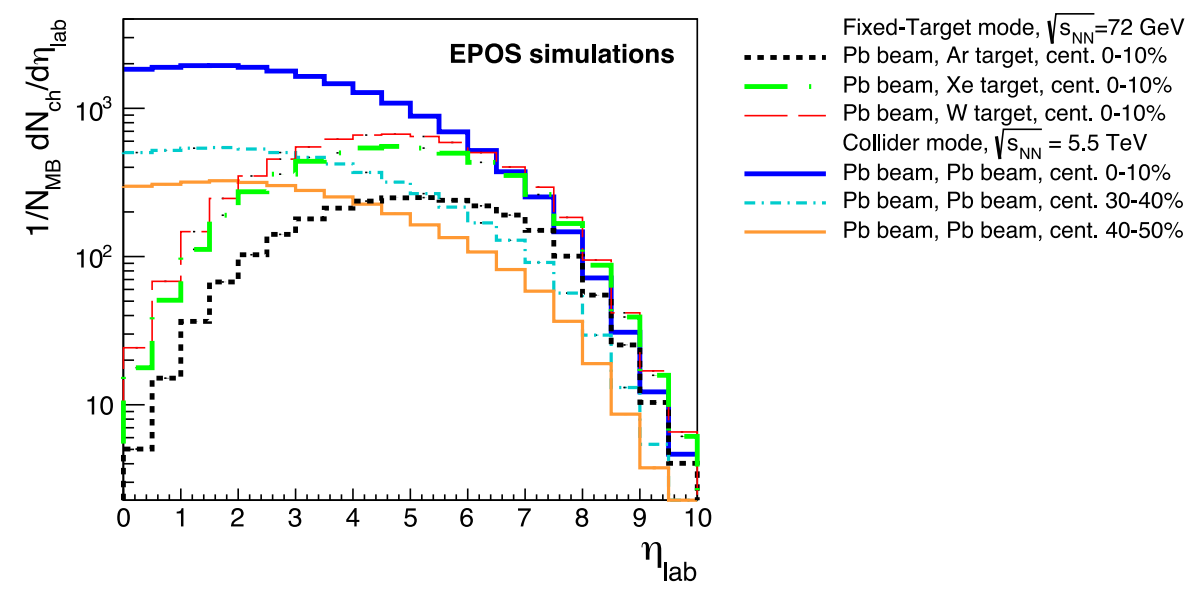

Fig. 9. The averaged charged-particle multiplicity as a function of the pseudo-rapidity in the laboratory frame for the most central collisions in PbAr, $\mathrm{PbXe}$ and $\mathrm{PbW}$ collisions at $\sqrt{s_{N N}}=72 \mathrm{GeV}$ in a fixed-target mode and for various event centrality intervals in PbPb collisions at $\sqrt{S_{N N}}=5.5 \mathrm{TeV}$ in a collider mode.

high-resolution vertex detector would allow one to measure precisely the primary and secondary vertices associated to the production of heavy-flavour hadrons. A polarised target requires space, e.g. for pumping system and diagnosis apparatus in the case of a gas target, and it is challenging, but possible, to couple it with a large angle detector.

The physics case comprises lead-nucleus collisions with instantaneous luminosity that can reach up to $3 \times 10^{28} \mathrm{~cm}^{-2} \mathrm{~s}^{-1}$ as well as proton-proton and proton-nucleus collisions with instantaneous luminosity up to $10^{33}$ $\mathrm{cm}^{-2} \mathrm{~s}^{-1}$. These numbers correspond to the maximum luminosities in each colliding system quoted in Table 9. The detectors must be able to cope with the occupancies and fluences for both of these configurations. In the case of the heaviest nuclear collisions foreseen, $\mathrm{PbXe}$ and $\mathrm{PbW}$ collisions at $\sqrt{S_{N N}}=72 \mathrm{GeV}$, the average number of charged particles is maximal at $\eta_{\text {lab. }} \sim 4.2$ and amounts to $d N_{c h} / d \eta \sim 600-700$ for the most $10 \%$ central collisions according to EPOS [114,159]. The charged-particle multiplicity is shown in Fig. 9 for various heavy-ion systems as a function of the pseudo-rapidity in the laboratory frame and is compared to the one obtained at the LHC in a collider mode. In a fixed-target mode, the multiplicity does not exceed the one obtained in PbPb collisions at $\sqrt{S_{N N}}=5.5 \mathrm{TeV}$ in a collider mode. If one considers the maximum instantaneous luminosities quoted in Table 9 and the inelastic cross-sections from EPOS ${ }^{18}$ one ends up with inelastic rates corresponding to $36 \mathrm{MHz}, 300 \mathrm{MHz}$ and $190 \mathrm{kHz}$ in $p p, p X e$ and $\mathrm{PbXe}$ collisions, respectively. These numbers are, for the $p p$ and $A A$ cases, of the same order of magnitude than the maximum rates planned for LHC in a collider mode in Run 3 and Run 4.

\subsection{Possible implementations with existing apparatus}

The proposed physics programme is rich and it is clear that building a completely new experiment would allow one to cope with the various requirements briefly detailed above. However one could already use an existing detector at the LHC in order to cover a large part of the physics programme. In this respect, the right panel of Fig. 10 shows the evolution of the rapidity coverage in the c.m.s. frame with $\sqrt{s_{N N}}$. The rapidity phase-space decreases while lowering the energy. While in a collider mode a forward-angle detector with $2<\eta<5$ covers approximately a forward-rapidity region of $2<y_{\text {c.m.s. }}<5$, in a fixed-target mode the same detector covers the mid-rapidity region as well as half of the backward-rapidity region. In the case of a proton beam of $7 \mathrm{TeV}$ on a fixed target, the rapidity coverage for the mentioned pseudo-rapidity range is $-2.8<y_{\text {c.m.s. }}<0.2$.

In the following sections, we will discuss possible implementations of the fixed-target programme at the LHC with two existing experiments: ALICE and LHCb. The detectors will be briefly presented in both cases as well as their upgrades planned for LHC Run 3 and 4 and we will discuss their ability to cover the physics programme described in this document. In the case of $\mathrm{LHCb}$, a fixed-target programme has recently started with a reduced luminosity and some aspects of the fixed-target mode will be described. For both experiments, the rapidity acceptance, the achievable luminosities as well as the physics reach will be discussed for various fixed-target systems and based on experimental constraints.

\footnotetext{
18 In order to compute the inelastic rate, we use the inelastic cross sections from EPOS, $\sigma_{\text {inel. }}=39 \mathrm{mb}$ in $p p$ collisions at $\sqrt{s}=115 \mathrm{GeV}, \sigma_{\text {inel. }}=$ $1.3 \mathrm{~b}$ in $p$ Xe collisions at $\sqrt{s_{N N}}=115 \mathrm{GeV}$ and $\sigma_{\text {inel. }}=6.2 \mathrm{~b}$ in PbXe collisions at $\sqrt{s_{N N}}=72 \mathrm{GeV}$, and the instantaneous luminosities of $10^{33} \mathrm{~cm}^{-2} \mathrm{~s}^{-1}$, $2 \times 10^{32} \mathrm{~cm}^{-2} \mathrm{~s}^{-1}$ and $3 \times 10^{28} \mathrm{~cm}^{-2} \mathrm{~s}^{-1}$ in $p p, p X e$ and PbXe collisions, respectively.
} 


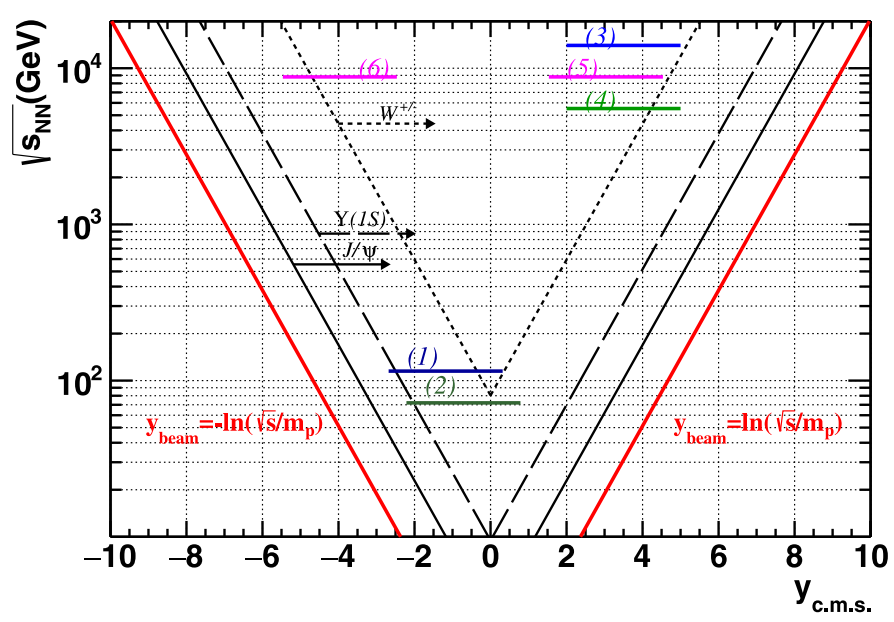

Fig. 10. The $y_{\text {c.m.s. }}$ coverage as a function of the colliding energies per nucleon pair $\left(\sqrt{S_{N N}}\right)$. The red solid lines represent the beam rapidity. The solid, dashed and dotted black lines respectively show the $y_{\text {c.m.s. }}$ coverage for the $J / \psi, \Upsilon(1 S)$ and $W^{+/-}$production. The horizontal lines show the $y_{\text {c.m.s. }}$ acceptance of a detector with a pseudo-rapidity coverage in the laboratory frame of $2<\eta<5$ for different colliding systems and modes using the $7 \mathrm{TeV}$ proton and $2.76 \mathrm{~A}$.TeV Pb LHC beams: (1) $p p$ and $p A$ collisions in the fixed-target mode at $\sqrt{s_{N N}}=115 \mathrm{GeV}$, (2) PbA collisions in the fixed-target mode at $\sqrt{s_{N N}}=72 \mathrm{GeV}$, (3) pp collisions in the collider mode at $\sqrt{\mathrm{s}}=14 \mathrm{TeV}$, (4) PbPb collisions in the collider mode at $\sqrt{s_{N N}}=5.5 \mathrm{TeV},(5) \mathrm{pPb}$ collisions in the collider mode at $\sqrt{s_{N N}}=8.8 \mathrm{TeV}$, (6) Pbp collisions in the collider mode at $\sqrt{s_{N N}}=8.8 \mathrm{TeV}$.

\subsubsection{ALICE as a fixed-target experiment}

The detectors of ALICE $[152,160]$ are optimised for studying the QCD matter created in high-energy collisions of lead nuclei. They are able to cope with high-multiplicity events and to track charged particles down to $p_{T} \sim 0.15 \mathrm{GeV} / \mathrm{c}$ at mid-rapidity.

The Central Barrel (CB) detectors are embedded into the L3 solenoid magnet that provides a field of $0.5 \mathrm{~T}$ parallel to the beam line. The inner most detector, the Inner Tracking System (ITS), tracks charged particles within $|\eta|<0.9$ and allows one to reconstruct primary and secondary vertices. The two innermost layers of the ITS cover $|\eta|<2$ and $|\eta|<1.4$ for the first and second layer, respectively. The resolution on the longitudinal position of the primary vertex ranges from 10 to $150 \mu \mathrm{m}$ decreasing with the charged-particle multiplicity. The Time Projection Chamber (TPC) provides track reconstruction as well as particle identification (PID) via the measurement of the specific ionisation energy loss $\mathrm{d} E / \mathrm{d} x$ in the gas volume. The phase space covered by the TPC in pseudo-rapidity is $|\eta|<0.9$ with full radial track length. The TPC acceptance can be extended by considering only $1 / 3$ of the full radial track length (also denoted as "TPC reduced track length" in the following) at the cost of worsening the momentum resolution. In that case, the pseudorapidity acceptance is $|\eta|<1.5$. The Time Of Flight (TOF) detector extends the PID via the measurement of the flight time of the charged particles from the Interaction Point (IP). Its pseudo-rapidity coverage is $|\eta|<0.9$. For that purpose the T0 detector located along the beam line measures the event collision time. The CB includes also High Momentum Particles Identification Detector (HMPID), calorimeters (Electromagnetic Calorimeter: EMCal and Photon Spectrometer: PHOS) and Transition Radiation Detector (TRD) for particle identification purpose. The transverse momentum relative resolution measured with both ITS and TPC ranges from 0.8 to $2 \%$ for $p_{T}=1$ to $10 \mathrm{GeV}$.

At forward rapidity, the Muon Spectrometer (MS) covers the pseudo-rapidity range $2.5<\eta<4$ in the laboratory frame. It includes a dipole magnet with an integrated field of $3 \mathrm{Tm}$, five tracking stations and two trigger stations. A system of absorbers located in front of the tracking and trigger stations and around the beam pipe is used for filtering out the hadrons and to protect the chambers from secondary particles produced during interactions of large- $\eta$ primary particles with the beam pipe. The combined effect of the front absorber and of the iron wall implies the detection of tracks matching the trigger chambers with $p>4 \mathrm{GeV}$. The relative muon momentum resolution is $\delta p / p \approx 1 \%$.

The ALICE upgrade [161] is scheduled for the second LHC Long Shutdown (LS2) that will take place in 2019, 2020 and 2021, and will exploit the LHC Run 3 and Run 4. In order to allow for a continuous readout at an interaction rate of $50 \mathrm{kHz}$ in $\mathrm{PbPb}$ collisions at $\sqrt{s_{N N}}=5.5 \mathrm{TeV}$, many detectors or their electronics will be upgraded. In $p p$ and $p A$ collisions, the detector upgrade will allow one to record data with a rate of $200 \mathrm{kHz}$. Fig. 58 presents a schematic view of the ALICE detectors for Run 3. A new detector, the Muon Forward Tracker (MFT), a Si-tracking detector, is designed to add vertexing capabilities to the MS by measuring charged tracks with a high spatial resolution. It is positioned along the beam axis between the ITS inner barrel and the MS front absorber. The MFT will cover the pseudo-rapidity acceptance $2.5<\eta<3.6$. The MFT capability to identify tracks coming from secondary vertices is measured by experimental resolution on the track offset to the primary vertex, the latter being measured by the ITS. Resolutions below 100 and $1000 \mu \mathrm{m}$ are found for $p_{T}>1 \mathrm{GeV}$ in the transverse and longitudinal direction, respectively. These resolutions decrease with increasing $p_{T}$ down to 25 and $180 \mu \mathrm{m}$ at large $p_{T}$ in the transverse and longitudinal direction, respectively. It is 


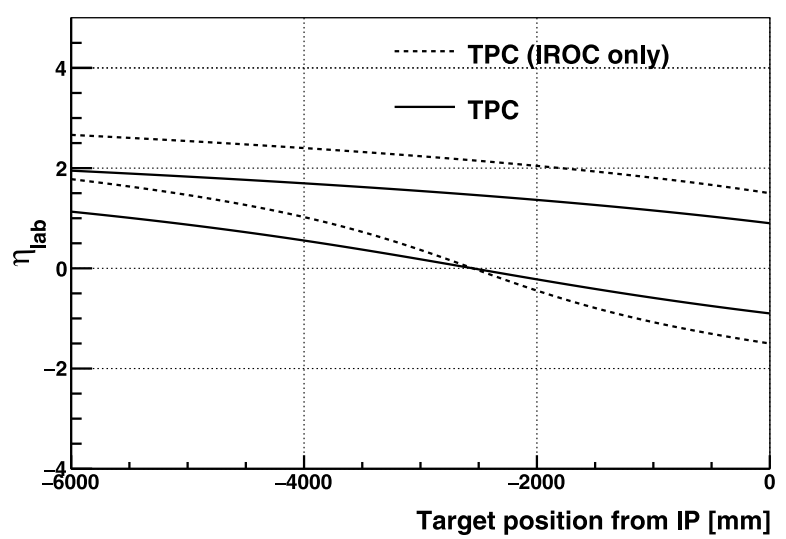

(a)

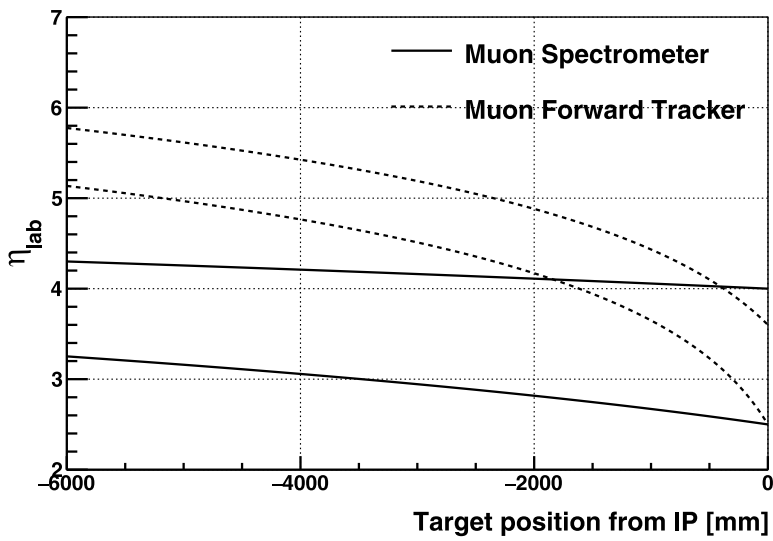

(b)

Fig. 11. (a) $\eta_{\text {lab. }}$ acceptance (between 2 curves) of the ALICE TPC as a function of the target position $\left(z_{\text {target }}\right)$ upstream from the nominal IP. Solid

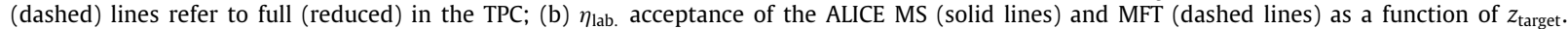

worth noting that the mass resolution will be greatly improved for the low-mass di-muon $\left(M_{\mu \mu}<1.5 \mathrm{GeV}\right)$ by adding the MFT to the MS. The mass resolution has been evaluated to be lower than $20 \mathrm{MeV}$ for $\eta, \phi$ and $\omega$ mesons.

In a fixed-target mode and with a target positioned at the IP, the acceptance of the MFT and MS allow for measurements in the rapidity regions of $-2.3<y_{\text {c.m.s. }}<-1.2$ with a $7 \mathrm{TeV}$ proton beam and of $-1.7<y_{\text {c.m.s. }}<-0.6$ with a 2.76 A.TeV Pb beam. The $\mathrm{CB}$ covers the very rear region with a centre-of-mass rapidity of $y_{\text {c.m.s. }}<-3.9$ and $y_{\text {c.m.s. }}<-3.3$, respectively, i.e. it allows one to access the very high- $x$ region. The target can also be displaced upstream of the nominal IP (on the A-side of ALICE, opposite to the MS). Fig. 11 shows the pseudo-rapidity acceptance of some ALICE detectors as a function of the target position, where the acceptance is computed considering the geometry of the active detectors. The acceptances are shifted towards the forward region when the target is displaced upstream of the nominal IP, in the opposite direction of the MS. If the target is displaced by a large amount and if one wants to measure the primary and secondary vertices precisely, a new vertex detector close to the target is then required. In case of a polarised target, its polarisation may be modified with the magnetic field of the L3 magnet. This brings additional constraints on the target position.

The occupancy of the $A A$ collision systems is not an issue in ALICE since the detectors were designed to measure $\mathrm{PbPb}$ collisions at $\sqrt{S_{N N}}=5.5 \mathrm{TeV}$ and the average charged-particle multiplicity in a fixed-target mode does not exceed the one in a collider mode as shown in Fig. 10.

As specified above, the detectors in ALICE will be upgraded in Run 3 in order to cope with an inelastic rate of $50 \mathrm{kHz}$ in $\mathrm{PbPb}$ collisions and $200 \mathrm{kHz}$ in $p p$ and $p A$ collisions, in a collider mode. The rate is essentially limited by the detector occupancy and, since similar occupancy is expected in $50 \mathrm{kHz} \mathrm{PbPb}$ and $4.5 \mathrm{MHz} p p$ collisions, it might be possible to run up to $1 \mathrm{MHz}$ in $p p$ and $p A$ collisions in a collider mode. This is under discussion in ALICE. In addition, in a fixed-target mode, a lower detector occupancy is expected and the rate will be limited by the occupancy in the MS acceptance, where the multiplicity is the largest. By scaling the average charged-particle multiplicities of the fixed-target to the collider mode in the MS acceptance, an increase in the ALICE readout rate by factors of about two in PbXe and ten in $p \mathrm{H}$ collisions, respectively, can be projected. Further studies are needed to demonstrate if such a higher rate is sustainable.

The MS and the MFT will cover the physics programme described above with the detection of single muon from heavyflavours, muon pairs (such as DY) and quarkonia down to low $p_{T}$. Further works are needed to estimate the level of background for the critical analyses such as DY in $A A$ collisions and in case the target is displaced upstream from the ALICE IP. The CB can detect and identify neutral and charged particles in the very backward region. Further studies will determine if the achievable luminosities allow one to complete some of the physics cases in this rear region.

There are ongoing feasibility studies on the installation of an internal solid target in the ALICE experiment [146]. The beam splitting option is currently investigated, where the beam halo is deflected by a crystal placed $\sim 70 \mathrm{~m}$ upstream from the nominal IP, and the deflected particles hit the target located inside the L3 magnet. The target holder is envisioned as an adjustable device, which facilitates moving the target from the parking position (outside of the beam pipe) to the working point, $13 \mathrm{~mm}$ from the beam axis [162]. The mechanical design of the system is under study. The target system could be integrated during LS3 at approximately $5 \mathrm{~m}$ from the interaction point (opposite side of the MS), in front of an existing valve that will be located at $4.8 \mathrm{~m}$ from the interaction point in Run 3 . The crystal and target devices could then be used in Run 4.

\subsection{2. $\mathrm{LHCb}$ as a fixed-target experiment}

The LHCb detector $[153,163]$ is a single-arm forward spectrometer, designed for studies of hadrons containing $b$ and/or $c$ quarks. Its pseudo-rapidity coverage in the laboratory frame is $2<\eta<5$. Such a geometrical coverage offers great 
opportunities when it is used in the fixed-target mode. It comprises a high precision tracking system, two ring-imaging Cherenkov detectors for the identification of different types of charged hadrons, a calorimeter system to identify photons, electrons and hadrons, and a muon system for the muon identification. The tracking system includes a silicon-strip vertex locator (VELO) and four stations with a dipole magnet between the first and the other three stations. It can achieve a relative momentum uncertainty of charged particles varying from $0.5 \%$ to $0.8 \%$ for the momentum between a few GeV to $100 \mathrm{GeV}$. The calorimeter system is composed of a scintillating pad, a preshower detector, an electromagnetic calorimeter, and a hadron calorimeter. The muon system consists of five muon stations with alternating layers of iron and multiwire proportional chambers. As described below, some detectors will be upgraded during the LS2 and the LS3, in order to exploit the LHC Run 3 and Run 4 periods. A schematic view of the upgraded detectors is shown in Fig. 59. The different subdetectors are as follows:

- VELO. The current VELO of LHCb is composed of 84 single-sided silicon strip sensors, operated in a secondary vacuum inside the LHC beam pipe [153]. The VELO length is about $1 \mathrm{~m}$ along the beam. The pitch of the $R$ sensors varies from 40 to $102 \mu \mathrm{m}$, and that of the $\phi$ sensors varies from 38 to $97 \mu \mathrm{m}$. The length of the shortest (longest) strip is $3.8 \mathrm{~mm}$ $(33.8 \mathrm{~mm}$ ) for R sensors; The length of the shortest (longest) strip is $5.9 \mathrm{~mm}(24.9 \mathrm{~mm})$ for $\phi$ sensors. The resolution of the reconstructed primary vertex is $13 \mu \mathrm{m}$ in the $x-y$ plane and $71 \mu \mathrm{m}$ in the $z$ direction, assuming that the number of tracks of the primary vertex is 25 . When the number of tracks reduces, the resolution becomes slightly worse. The resolution of the impact parameter is about $15-50 \mu \mathrm{m}[100,163]$. The excellent vertex reconstruction ability allows one to well separate the primary vertex and the secondary vertex of B or charmed hadron decays. For the LHCb upgrade during the LS2, the current VELO detector will be completely replaced by a new detector based on hybrid silicon pixel sensors [164]. The pixel pitch is $50 \mu \mathrm{m} \times 50 \mu \mathrm{m}$. It will have the same physics performance and can deliver a readout at $40 \mathrm{MHz}$. Compared to the current silicon strip VELO, the new VELO can cope with events with much higher track multiplicity.

- Tracking. The first station of the current tracking system is based on silicon micro-strip. The other three stations, which are located after the $\mathrm{LHCb}$ dipole magnet, are composed of silicon micro-strip inner trackers and straw drift tube outer trackers. The relative momentum resolution is about $0.5 \%-1 \%$. The mass resolution of $\mathrm{K}_{\mathrm{s}}^{0}$ mesons is 3.5 (7) MeV if they decay inside (outside) the VELO. For the LHCb upgrade, the first station will be replaced by high granularity silicon micro-strip planes, and the other stations will be replaced by scintillating fibre trackers [165]. The momentum resolution will be about $10 \%-20 \%$ better than the current resolution.

- Calorimeter. The electromagnetic calorimeter is composed of a sampling scintillator-lead structure. The hadron calorimeter is a sampling scintillator-iron structure. The mass resolution of low transverse momentum $\pi^{0}$ mesons, reconstructed with well-separated photons, is $8 \mathrm{MeV}$. For $\pi^{0}$ mesons with transverse momentum greater than $2 \mathrm{GeV}$, the mass resolution is around 20 (30) MeV for those reconstructed with well separated (merged) photons. If one uses converted photons, the resolution of the mass difference between $M\left(\mu^{+} \mu^{-} \gamma\right)$ and $M\left(\mu^{+} \mu^{-}\right)$is around $5 \mathrm{MeV}$ for $\chi_{c}$ states. LHCb is now discussing an upgrade of the calorimeter that would occur in LS3 and LS4 [166], replacing the current electromagnetic calorimeter by a silicon-tungsten sampling calorimeter.

- Muon system. The muon system includes five rectangular shaped stations. The first station is made of triple Gas Electron Multiplier detectors, while the other four are composed of multiwire proportional chambers. The di-muon invariant-mass resolution is about $14(43) \mathrm{MeV}$ at the $J / \psi(\Upsilon(1 S))$ mass. The muon identification efficiency is above $95 \%$ for the tracks with transverse momentum above $1.7 \mathrm{GeV}$.

- Readout. The current $\mathrm{LHCb}$ detector reduces the event rate from $40 \mathrm{MHz}$ to $1 \mathrm{MHz}$ at the first level hardware trigger. After the LHC LS2, the upgraded hardware trigger will have the capability to read the full event information at a rate up to $40 \mathrm{MHz}$.

- HERSCHEL detector [167].

LHCb installed a HeRSCHEL (High Rapidity Shower Counters for LHCb) subdetector for Run 2 of the LHC. It is a system of forward shower counters consisting of five scintillator planes with PMTs. These five stations are installed perpendicular to the beam, their $z$ coordinates are $-114 \mathrm{~m},-19.7 \mathrm{~m},-7.5 \mathrm{~m},+20 \mathrm{~m}$, and $+114 \mathrm{~m}$, respectively. The $z$ direction of the coordinate system is from the VELO to the muon system along the beam, and the origin is the interaction point inside the VELO. Combining with other LHCb subdetectors, HERSCHEL greatly extends the sensitivity to detect charged particles at high pseudo-rapidities: $-10<\eta<-5,-3.5<\eta<-1.5,2<\eta<5$, and $5<\eta<10$.

The capability of LHCb to cope with high-multiplicity events and the limit on the event charged track multiplicity will be defined with the ongoing data reconstruction of PbPb collisions at $5 \mathrm{TeV}$ and $\mathrm{PbAr}$ collisions at $69 \mathrm{GeV}$. The occupancy of the VELO is essential to determine the track reconstruction, since the VELO provides the best position precision among all subdetectors of the tracking system. As shown in Fig. 19 of Ref. [100], the cluster occupancy in the current VELO varies from $0.4 \%$ to $0.6 \%$ depending on the positions of the silicon strips. This result is obtained with a data sample passing a random trigger on beam crossing, and the average number of visible interactions per beam crossing is $\mu=1.7$. The 


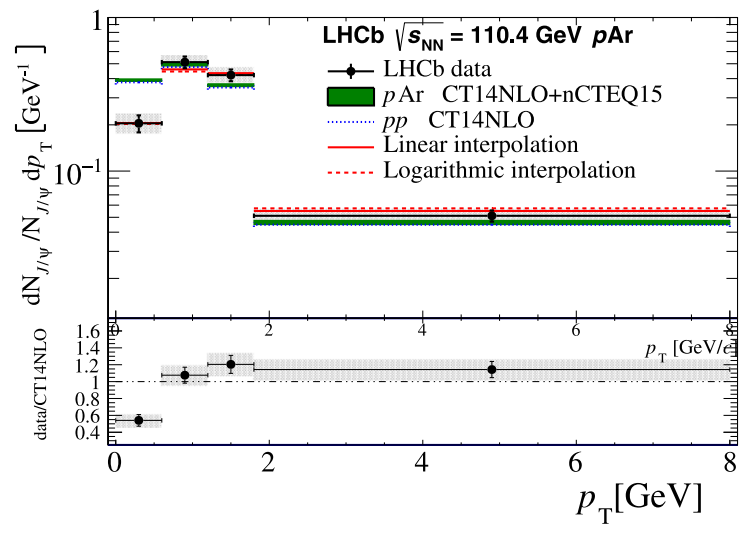

(a)

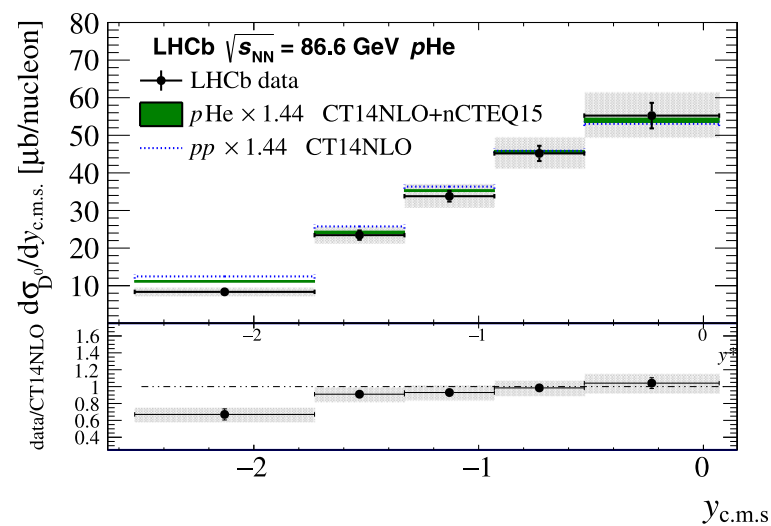

(b)

Fig. 12. (a) $J / \psi$ yield as a function of transverse momentum in pAr collisions. (b) $D$ meson cross-section as a function of centre-of-mass rapidity in pHe collisions. In both cases, the data are collected by the LHCb detector in the fixed-target mode. Source: Adapted from [168].

occupancy of the upgraded VELO will be significantly reduced owing to the replacement of silicon strips by hybrid silicon pixels. As shown in Fig. 20 of Ref. [164], the cluster occupancy is $0.08 \%$ for the pixels closest to the IP (0.5 mm). It drops rapidly below $0.01 \%$ as the radius increases. This result is obtained using simulated minimum-bias events at $\mu=5.2$. When the difference of the data samples is taken into account, the occupancy of the upgraded VELO is expected to be reduced by a factor of approximately 20 .

As discussed in Section 3.3.1, since the pilot runs of pNe and PbNe in 2012 and 2013, the direct injection system SMOG [112] is used in the fixed-target mode by injecting different gases inside the VELO vessel. For the 6.5 TeV proton beams, only special runs, like van der Meer scans or the period corresponding to the ramp up of the beam energy, were used. The time duration of $p \mathrm{He}, p \mathrm{Ne}$ and $p$ Ar collisions was typically $10-20 \mathrm{~h}$ for each year, respectively. For $2.5 \mathrm{TeV}$ proton beams, the proton-gas data were taken in parallel with $p p$ collisions; the time duration of $p$ He collisions was around $100 \mathrm{~h}$, and that of $p \mathrm{Ne}$ was around 200 hours. Only Neon and Argon gases were used with lead beams. During the PbPb LHC run in 2015, PbAr data were taken with a time duration of approximately $50 \mathrm{~h}$.

Even though the data taking time was limited, SMOG delivered physics results [116,168]. The LHCb collaboration reported the anti-proton differential-cross-section measurement in $p \mathrm{He}$ collisions and the $p \mathrm{Ar}$ run demonstrated the LHCb capabilities for charmed meson and charmonium reconstruction in the fixed-target mode. Both the $J / \psi \rightarrow \mu^{+} \mu^{-}$and $D^{0} \rightarrow K^{\mp} \pi^{ \pm}$were measured with an excellent mass resolution and an adequate efficiency [117]. Fig. 12 shows the ratio of $J / \psi$ and $D^{0}$ yields evaluated in pAr collisions at $\sqrt{S_{N N}}=110 \mathrm{GeV}$.

When the SMOG data were taken in parallel with $p p$ or PbPb collisions, the trigger of the SMOG related events was designed to fully utilise the data acquisition (DAQ) potential. When the DAQ busy time was too large, tighter cuts were used for the SMOG trigger.

In a fixed-target mode and with a target positioned at the IP, the LHCb detectors probe a rapidity region of $-2.7<$ $y_{\text {c.m.s. }}<0.3$ with a $7 \mathrm{TeV}$ proton beam and of $-2.2<y_{\text {c.m.s. }}<0.8$ with a $2.76 \mathrm{~A}$.TeV Pb beam. If the target is shifted by $0.4(1.5) \mathrm{m}$ on the opposite side of the spectrometer, one obtains the following acceptance by simply considering the active parts of the detectors including the VELO: $-2.7<y_{\text {c.m.s. }}<1.3\left(-0.7<y_{\text {c.m.s. }}<1.8\right)$ with the proton beam and of $-2.2<y_{\text {c.m.s. }}<1.8\left(-0.2<y_{\text {c.m.s. }}<2.3\right)$ with the Pb beam.

Studies were carried out for the installation of polarised and unpolarised high-density gaseous target in the LHCb experiment [146]. It was proposed to inject the gases in a storage cell (as described in Section 3.3.3) attached to the end of the VELO RF shields and located at $0.4 \mathrm{~m}$ from the nominal IP. This project, denoted SMOG2 [169,170], would significantly increase the luminosity, by up to a factor of 100. A baseline scenario was discussed in [171] for Run 3 with the following integrated luminosities: $\mathcal{L}_{p H}=150 \mathrm{pb}^{-1}, \mathcal{L}_{p D}=9 \mathrm{pb}^{-1}, \mathcal{L}_{p A r}=45 \mathrm{pb}^{-1}, \mathcal{L}_{p K r}=30 \mathrm{pb}^{-1} \mathrm{and} \mathcal{L}_{p X e}=$ $22 \mathrm{pb}^{-1}$ at $\sqrt{s_{N N}}=115 \mathrm{GeV}$ as well as $\mathcal{L}_{P b A r}=50 \mathrm{nb}^{-1}, \mathcal{L}_{P b H}=10 \mathrm{nb}^{-1}$ and $\mathcal{L}_{p A r}=5 \mathrm{pb}^{-1}$ at $\sqrt{s_{N N}}=72 \mathrm{GeV}$. SMOG2 will be a first step towards the fixed-target programme described in this paper. The storage cell was also proposed to be used with a polarised set-up [172] with an atomic-beam source, a gas diagnosis system and a magnet that would provide a $0.3 \mathrm{~T}$ field, transversally to the beam axis. In this case, the target position has to be defined according to the space availability in front of the VELO vessel. The target cannot be inside the VELO vessel because of the high gas flow that requires differential pumping system in a separate target chamber. Studies are ongoing with a target displaced by $1-2 \mathrm{~m}$ from the IP in front of a sector valve which isolates the target chamber from the VELO [173]. In these studies, a vertex detector is proposed behind the target. An installation of such a polarised target system is not foreseen before LS3. 


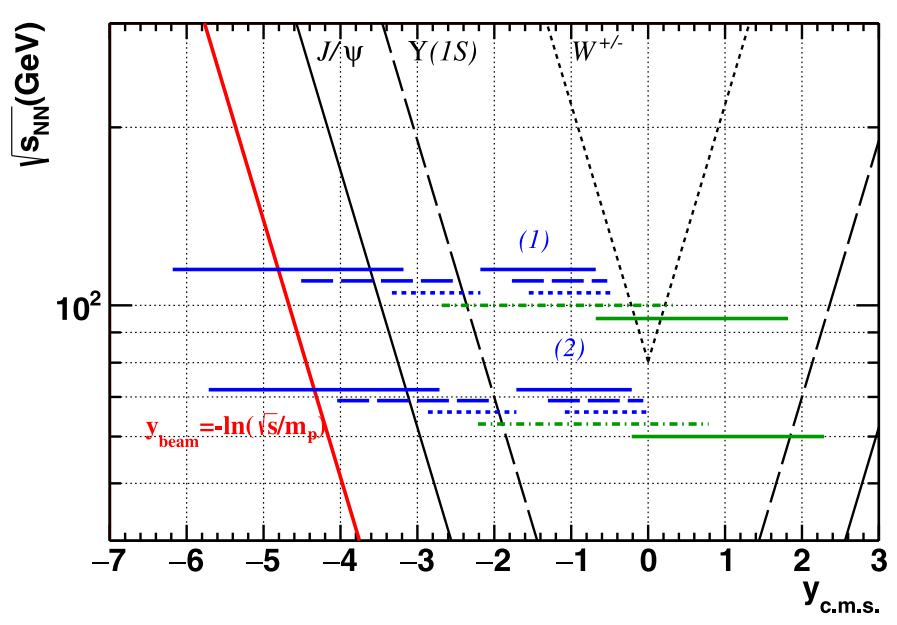

Fig. 13. Centre-of-mass-rapidity ( $\left.y_{\text {c.m.s. }}\right)$ coverage as a function of the colliding energies per nucleon pair $\left(\sqrt{s_{N N}}\right)$ as in Fig. 10 . The blue lines represent the acceptance of the TPC and MS of ALICE. The full, long-dashed and short-dashed lines correspond to targets located at the IP, upstream of the $\mathrm{IP}$, at $z_{\text {target }}=-2.75$ and $-4.7 \mathrm{~m}$, respectively. The dash-dotted green lines represent the acceptance of the LHCb detector with a target at the IP and the full green lines with a target upstream of the IP by $z_{\text {target }}=1.5 \mathrm{~m}$. The long-dashed and short-dashed blue lines as well as the dash-dotted and full green lines are shifted in energy for a better visibility.

\subsubsection{Comparison of possible implementations}

Fig. 13 shows the $y_{\text {c.m.s. }}$ acceptances of the ALICE and LHCb detectors for two fixed-target energies, namely $\sqrt{s_{N N}}=72$ $\mathrm{GeV}$ and $115 \mathrm{GeV}$. The rapidity coverages with a target position at the nominal IP are shown as full line for ALICE and dash-dotted line for LHCb. The latter corresponds to the case of LHCb used as a fixed-target detector with the SMOG system. The acceptances are also shown with two (one) other target positions for ALICE (LHCb), where the acceptance is determined considering the geometry of the active part of the detector as detailed in Section 4.2.1 and 4.2.2. For a target located at the IP, the ALICE MS and the LHCb detectors cover the central $y_{\text {c.m.s. }}$ region as well as half of the backward rapidity acceptance $\left(y_{\text {c.m.s. }}<0\right)$, while the ALICE CB has the particularity to probe the target-rapidity region and the end of the phase space $\left(x_{2} \rightarrow 1\right.$ and $\left.x_{F} \rightarrow-1\right)$. When the target is shifted on the opposite side of the detector, the rapidity interval probed is shifted forward. In the ALICE and LHCb cases, the wide $y_{\text {c.m.s. }}$ range makes these detectors suitable to study the rapidity dependence of various probes.

Tables 11 and 12 show the achievable luminosities using the ALICE and LHCb detectors during one LHC year, if one considers as a limitation the aforementioned experimental data-taking rates in the collider mode and by considering the luminosities of Table 9. As specified in Section 4.2.1, a higher rate could be envisioned in some cases since the chargedparticle multiplicity is lower in the fixed-target mode. We have also assumed that ALICE and LHCb could run in the fixed-target mode during the full year with proton $\left(10^{7} \mathrm{~s}\right)$ and lead beams $\left(10^{6} \mathrm{~s}\right)$ with the corresponding instantaneous luminosities. In some cases, namely the gas-jet, the storage-cell and the solid target coupled to the beam splitting by a crystal, the resulting interaction rates are high and close to those expected in the collider mode for the LHC Runs 3 and 4. Additional limitations will arise from various constraints such as the disk storage, the high-level trigger, the radiation level, the simultaneous running with the collider mode, etc. The Run 3 and Run 4 programmes for ALICE and LHCb are well settled in the collider mode and any fixed-target-running scenario will have to comply with these programmes. These constraints are not discussed in this review as they deserve dedicated studies within the experiments. In the following, we discuss the luminosities obtained with the three technical implementations in both the ALICE and LHCb set-ups and comment on the luminosity needs for some of the physics cases that will be described in Sections 5.1-5.3.

With $\mathrm{LHCb}$, the luminosity reach with a proton beam on an hydrogen target can be very large, up to yearly luminosity of the order of $10 \mathrm{fb}^{-1}$, if one can run at $40 \mathrm{MHz}$ for a full LHC year. As discussed in Section 5.1, this will allow one to measure hard probes such as $W$ or associated $J / \psi$ production, to collect very large statistics for DY and probe the $D$ meson production at the most backward rapidity range. For example, DY measurements are very useful to probe the light quark and anti-quark PDFs at high- $x$ and a low scale, $\mu_{F}$, even with a reduced luminosity of $1 \mathrm{fb}^{-1}$. By using nuclear solid or gas targets, one can reach luminosities on the order of $100 \mathrm{pb}^{-1}$, and even more depending on the target option, allowing one to probe the nuclear PDFs with a very high precision by measuring DY, open heavy flavour and quarkonium production. With proton and lead beams, the luminosity reach for ALICE is lower than for LHCb because of the lower data-taking rates. However, the rapidity coverage is complementary as well as the physics reach. Even though with ALICE the yearly luminosity can be as high as $250 \mathrm{pb}^{-1}$ with a proton beam on a $\mathrm{H}$-gas target, a luminosity of about $40 \mathrm{pb}^{-1}$ with the ALICE detectors would already allow one to measure low energy $\bar{p}$, thanks to the very backward rapidity coverage, in $p+p \rightarrow \bar{p}+X$. This measurement would improve our knowledge of the cosmic $\bar{p}$ spectrum. The $\bar{p}$ spectrum could be further measured with various target types, such as He or $\mathrm{C}$ with the gas or solid target option, respectively. 
Table 11

Summary table of the achievable integrated luminosities with the ALICE detector accounting for the data-taking-rate capabilities in the collider mode and by considering the luminosities of Table 9 . As detailed in the text, a higher inelastic rate $\left(\Gamma_{\text {inel. }}\right)$ depending on the collision system could be envisioned. The inelastic cross sections $\left(\sigma_{\text {inel. }}\right)$ are taken from EPOS [114,159].

\begin{tabular}{|c|c|c|c|c|c|c|c|c|c|c|}
\hline \multirow[t]{3}{*}{ Target } & & & \multicolumn{8}{|l|}{ ALICE } \\
\hline & & & \multicolumn{4}{|c|}{ Proton beam $\left(\sqrt{S_{N N}}=115 \mathrm{GeV}\right)$} & \multicolumn{4}{|c|}{$\mathrm{Pb}$ beam $\left(\sqrt{s_{N N}}=72 \mathrm{GeV}\right)$} \\
\hline & & & $\begin{array}{l}\mathcal{L} \\
{\left[\mathrm{cm}^{-2} \mathrm{~s}^{-1}\right]}\end{array}$ & $\begin{array}{l}\sigma_{\text {inel. }} \\
{[\mathrm{mb}]}\end{array}$ & $\begin{array}{l}\Gamma_{\text {inel. }} \\
{[\mathrm{kHz}]}\end{array}$ & $\int \mathcal{L}$ & $\begin{array}{l}\mathcal{L} \\
{\left[\mathrm{cm}^{-2} \mathrm{~s}^{-1}\right]}\end{array}$ & $\begin{array}{l}\sigma_{\text {inel. }} \\
{[\mathrm{b}]}\end{array}$ & $\begin{array}{l}\Gamma_{\text {inel. }} \\
{[\mathrm{kHz}]}\end{array}$ & $\int_{\left[\mathrm{nb}^{-1}\right]}^{\mathcal{L}}$ \\
\hline \multirow{10}{*}{$\begin{array}{l}\text { Internal gas } \\
\text { target }\end{array}$} & \multirow{5}{*}{ Gas-Jet } & $\mathrm{H}^{\uparrow}$ & $4.3 \times 10^{30}$ & 39 & 168 & 43 & $5.6 \times 10^{26}$ & 1.8 & 1.0 & $5.6 \times 10^{-1}$ \\
\hline & & $\mathrm{H}_{2}$ & $2.6 \times 10^{31}$ & 39 & 1000 & $2.6 \times 10^{2}$ & $2.8 \times 10^{28}$ & 1.8 & 50 & 28 \\
\hline & & $\mathrm{D}^{\uparrow}$ & $4.3 \times 10^{30}$ & 72 & 309 & 43 & $5.6 \times 10^{26}$ & 2.2 & 1.2 & $5.6 \times 10^{-1}$ \\
\hline & & ${ }^{3} \mathrm{He}^{\uparrow}$ & $8.5 \times 10^{30}$ & 117 & 1000 & 85 & $2.0 \times 10^{28}$ & 2.5 & 50 & 20 \\
\hline & & $\mathrm{Xe}$ & $7.7 \times 10^{29}$ & 1300 & 1000 & 7.7 & $8.1 \times 10^{27}$ & 6.2 & 50 & 8.1 \\
\hline & \multirow{5}{*}{ Storage Cell } & $\mathrm{H}^{\uparrow}$ & $2.6 \times 10^{31}$ & 39 & 1000 & $2.6 \times 10^{2}$ & $2.8 \times 10^{28}$ & 1.8 & 50 & 28 \\
\hline & & $\mathrm{H}_{2}$ & $2.6 \times 10^{31}$ & 39 & 1000 & $2.6 \times 10^{2}$ & $2.8 \times 10^{28}$ & 1.8 & 50 & 28 \\
\hline & & $\mathrm{D}^{\uparrow}$ & $1.4 \times 10^{31}$ & 72 & 1000 & $1.4 \times 10^{2}$ & $2.2 \times 10^{28}$ & 2.2 & 50 & 22 \\
\hline & & ${ }^{3} \mathrm{He}^{\uparrow}$ & $8.5 \times 10^{30}$ & 117 & 1000 & 85 & $2.0 \times 10^{28}$ & 2.5 & 50 & 20 \\
\hline & & $\mathrm{Xe}$ & $7.7 \times 10^{29}$ & 1300 & 1000 & 7.7 & $8.1 \times 10^{27}$ & 6.2 & 50 & 8.1 \\
\hline \multirow{4}{*}{$\begin{array}{l}\text { Internal solid } \\
\text { target on beam } \\
\text { halo }\end{array}$} & \multirow{4}{*}{ Wire target } & $\mathrm{C}(500 \mu \mathrm{m})$ & $2.8 \times 10^{30}$ & 271 & 760 & 28 & $5.6 \times 10^{26}$ & 3.3 & 1.8 & $5.6 \times 10^{-1}$ \\
\hline & & $\mathrm{Ti}(500 \mu \mathrm{m})$ & $1.4 \times 10^{30}$ & 694 & 971 & 14 & $2.8 \times 10^{26}$ & 4.7 & 1.3 & $2.8 \times 10^{-1}$ \\
\hline & & $\mathrm{W}(184 \mu \mathrm{m})$ & $5.9 \times 10^{29}$ & 1700 & 1000 & 5.9 & - & - & - & - \\
\hline & & $\mathrm{W}(500 \mu \mathrm{m})$ & - & - & - & - & $3.1 \times 10^{26}$ & 6.9 & 2.1 & $3.1 \times 10^{-1}$ \\
\hline \multirow{8}{*}{ Beam splitting } & \multirow{2}{*}{ E1039 } & $\mathrm{NH}_{3}^{\uparrow}$ & $2.4 \times 10^{30}$ & 420 & 1000 & 24 & $2.7 \times 10^{27}$ & 19 & 50 & 2.7 \\
\hline & & $\mathrm{ND}_{3}^{\uparrow}$ & $1.9 \times 10^{30}$ & 519 & 1000 & 19 & $2.2 \times 10^{27}$ & 22 & 50 & 2.2 \\
\hline & \multirow{6}{*}{$\begin{array}{l}\text { Unpolarised } \\
\text { solid target }\end{array}$} & $\mathrm{C}(658 \mu \mathrm{m})$ & $3.7 \times 10^{30}$ & 271 & 1000 & 37 & - & - & - & - \\
\hline & & $\mathrm{C}(5 \mathrm{~mm})$ & - & - & - & - & $5.6 \times 10^{27}$ & 3.3 & 18 & 5.6 \\
\hline & & $\mathrm{Ti}(515 \mu \mathrm{m})$ & $1.4 \times 10^{30}$ & 694 & 1000 & 14 & - & - & - & - \\
\hline & & $\mathrm{Ti}(5 \mathrm{~mm})$ & - & - & - & - & $2.8 \times 10^{27}$ & 4.7 & 13 & 2.8 \\
\hline & & $\mathrm{W}(184 \mu \mathrm{m})$ & $5.9 \times 10^{29}$ & 1700 & 1000 & 5.9 & - & - & - & - \\
\hline & & $\mathrm{W}(5 \mathrm{~mm})$ & - & - & - & - & $3.1 \times 10^{27}$ & 6.9 & 21 & 3.1 \\
\hline
\end{tabular}

Table 12

Same as Table 11 for the LHCb detector.

\begin{tabular}{|c|c|c|c|c|c|c|c|c|c|c|}
\hline \multirow[t]{3}{*}{ Target } & & & \multicolumn{8}{|l|}{ LHCb } \\
\hline & & & \multicolumn{4}{|c|}{ Proton beam $\left(\sqrt{s_{N N}}=115 \mathrm{GeV}\right)$} & \multicolumn{4}{|c|}{$\mathrm{Pb}$ beam $\left(\sqrt{s_{N N}}=72 \mathrm{GeV}\right)$} \\
\hline & & & $\begin{array}{l}\mathcal{L} \\
{\left[\mathrm{cm}^{-2} \mathrm{~s}^{-1}\right]}\end{array}$ & $\begin{array}{l}\sigma_{\text {inel. }} \\
{[\mathrm{mb}]}\end{array}$ & $\begin{array}{l}\Gamma_{\text {inel. }} \\
{[\mathrm{kHz}]}\end{array}$ & $\int_{\left[\mathrm{pb}^{-1}\right]}^{\mathcal{L}}$ & $\begin{array}{l}\mathcal{L} \\
{\left[\mathrm{cm}^{-2} \mathrm{~s}^{-1}\right]}\end{array}$ & $\begin{array}{l}\sigma_{\text {inel. }} \\
{[\mathrm{mb}]}\end{array}$ & $\begin{array}{l}\Gamma_{\text {inel. }} \\
{[\mathrm{kHz}]}\end{array}$ & $\int_{\left[\mathrm{nb}^{-1}\right]}^{\mathcal{L}}$ \\
\hline \multirow{10}{*}{$\begin{array}{l}\text { Internal gas } \\
\text { target }\end{array}$} & \multirow{5}{*}{ Gas-Jet } & $\mathrm{H}^{\uparrow}$ & $4.3 \times 10^{30}$ & 39 & 168 & 43 & $5.6 \times 10^{26}$ & 1.8 & 1 & $5.6 \times 10^{-1}$ \\
\hline & & $\mathrm{H}_{2}$ & $1.0 \times 10^{33}$ & 39 & 40000 & $1.0 \times 10^{4}$ & $1.2 \times 10^{29}$ & 1.8 & 212 & $1.2 \times 10^{2}$ \\
\hline & & $\mathrm{D}^{\uparrow}$ & $4.3 \times 10^{30}$ & 72 & 309 & 43 & $5.6 \times 10^{26}$ & 2.2 & 1 & $5.6 \times 10^{-1}$ \\
\hline & & ${ }^{3} \mathrm{He}^{\uparrow}$ & $3.4 \times 10^{32}$ & 117 & 40000 & $3.4 \times 10^{3}$ & $4.7 \times 10^{28}$ & 2.5 & 118 & 47 \\
\hline & & $\mathrm{Xe}$ & $3.1 \times 10^{31}$ & 1300 & 40000 & $3.1 \times 10^{2}$ & $2.3 \times 10^{28}$ & 6.2 & 186 & 23 \\
\hline & \multirow{5}{*}{ Storage cell } & $\mathrm{H}^{\uparrow}$ & $9.2 \times 10^{32}$ & 39 & 35880 & $9.2 \times 10^{3}$ & $1.2 \times 10^{29}$ & 1.8 & 212 & $1.2 \times 10^{2}$ \\
\hline & & $\mathrm{H}_{2}$ & $1.0 \times 10^{33}$ & 39 & 40000 & $1.0 \times 10^{4}$ & $1.2 \times 10^{29}$ & 1.8 & 212 & $1.2 \times 10^{2}$ \\
\hline & & $D^{\uparrow}$ & $5.6 \times 10^{32}$ & 72 & 40000 & $5.6 \times 10^{3}$ & $8.8 \times 10^{28}$ & 2.2 & 194 & 88 \\
\hline & & ${ }^{3} \mathrm{He}^{\uparrow}$ & $1.3 \times 10^{33}$ & 117 & 40000 & $1.3 \times 10^{4}$ & $8.3 \times 10^{28}$ & 2.5 & 206 & 83 \\
\hline & & $\mathrm{Xe}$ & $3.1 \times 10^{31}$ & 1300 & 40000 & $3.1 \times 10^{2}$ & $3.0 \times 10^{28}$ & 6.2 & 186 & 30 \\
\hline \multirow{3}{*}{$\begin{array}{l}\text { Internal solid } \\
\text { target on beam } \\
\text { halo }\end{array}$} & \multirow{3}{*}{ Wire target } & $\mathrm{C}(500 \mu \mathrm{m})$ & $2.8 \times 10^{30}$ & 271 & 760 & 28 & $5.6 \times 10^{26}$ & 3.3 & 2 & $5.6 \times 10^{-1}$ \\
\hline & & $\mathrm{Ti}(500 \mu \mathrm{m})$ & $1.4 \times 10^{30}$ & 694 & 972 & 14 & $2.8 \times 10^{26}$ & 4.7 & 1 & $2.8 \times 10^{-1}$ \\
\hline & & $\mathrm{W}(500 \mu \mathrm{m})$ & $1.6 \times 10^{30}$ & 1700 & 2720 & 16 & $3.1 \times 10^{26}$ & 6.9 & 2 & $3.1 \times 10^{-1}$ \\
\hline \multirow{5}{*}{ Beam splitting } & \multirow{2}{*}{ E1039 } & $\mathrm{NH}_{3}^{\uparrow}$ & $7.2 \times 10^{31}$ & 420 & 30240 & $7.2 \times 10^{2}$ & $1.4 \times 10^{28}$ & 19 & 259 & 14 \\
\hline & & $\mathrm{ND}_{3}^{\uparrow}$ & $7.2 \times 10^{31}$ & 519 & 37368 & $7.2 \times 10^{2}$ & $1.4 \times 10^{28}$ & 22 & 314 & 14 \\
\hline & \multirow{3}{*}{$\begin{array}{l}\text { Unpolarised } \\
\text { solid target }\end{array}$} & $\mathrm{C}(5 \mathrm{~mm})$ & $2.8 \times 10^{31}$ & 271 & 7600 & $2.8 \times 10^{2}$ & $5.6 \times 10^{27}$ & 3.3 & 18 & 5.6 \\
\hline & & Ti $(5 \mathrm{~mm})$ & $1.4 \times 10^{31}$ & 694 & 9720 & $1.4 \times 10^{2}$ & $2.8 \times 10^{27}$ & 4.7 & 13 & 2.8 \\
\hline & & $\mathrm{W}(5 \mathrm{~mm})$ & $1.6 \times 10^{31}$ & 1700 & 27200 & $1.6 \times 10^{2}$ & $3.1 \times 10^{27}$ & 6.9 & 21 & 3.1 \\
\hline
\end{tabular}

With a transversally polarised H-gas target, luminosities from about $40 \mathrm{pb}^{-1}$ to $250 \mathrm{pb}^{-1}$ can be expected with ALICE for one LHC year. As discussed in Section 5.2, this would allow one to access the spin asymmetry of probes such as $\Lambda$ in the $\mathrm{CB}$ and $J / \psi$ in the MS. With a larger luminosity in $\mathrm{LHCb}$, on the order of $10 \mathrm{fb}^{-1}$, similar rare probes as those mentioned above for the unpolarised case $(W$, associated $J / \psi$, DY, ...) will be accessible. These studies will definitely advance our understanding of the internal spin structure of the proton and neutron. 
With a $\mathrm{Pb}$ beam on a heavy nuclear target, the luminosity is mainly limited by the impact on the beam lifetime for the gas target and by the useable beam flux for the beam splitting case. The luminosities do not differ by more than a factor of four between ALICE $\left(\mathcal{L}=8 \mathrm{nb}^{-1}\right)$ and $\operatorname{LHCb}\left(\mathcal{L}=30 \mathrm{nb}^{-1}\right)$ for PbXe. In the case of the beam splitting option, large luminosities are also expected $\left(\mathcal{L}=3 \mathrm{nb}^{-1}\right)$ for $\mathrm{PbW}$. When the nuclear target is lighter, the $\mathrm{Pb}$ beam lifetime is less affected and the luminosities are larger for the case of $\mathrm{LHCb}$ coupled to a gas target. A full programme of heavy-ion studies can be carried out in the fixed-target mode at the LHC in particular with precise quarkonium measurements, with studies of the heavy-quark energy-loss mechanism and a rapidity scan of the yield and elliptic flow of identified charged particles over a broad rapidity range, as discussed in Section 5.3. These studies can be performed with large statistics in $\mathrm{PbA}$ collisions but also in $p p$ and $p A$ collisions (see e.g. Table 20 for the $\Upsilon$ case), with the ALICE and LHCb detectors. In the latter case, it is not yet established up to which event centrality the tracks can efficiently be reconstructed, in particular for the heaviest nuclear target such as Xe. As discussed in Section 4.2.2, on-going studies will give more information on the event-centrality reach with LHCb for the Run 3 and Run 4 . A heavy-ion programme is already envisioned in LHCb in Run 3 with Ar gas target that will allow one to study soft probes as well as hard probes such as open heavy flavour and quarkonium production.

\section{Physics projections}

In this section, we review the projected performances for each of the 3 main topics. The assumptions considered for the generation of pseudo-data for LHCb and ALICE will be given. Figures-of-Merits (FoM) will refer to AFTER@LHCb, AFTER@ALICE $_{\mu}$ or AFTER@ALICE ${ }_{\mathrm{CB}}$ for pseudo-data generated in the acceptance of LHCb, the ALICE Muon Spectrometer and the ALICE Central Barrel, respectively.

\subsection{High-x frontier for particle and astroparticle physics}

The purpose of this section is to address the question whether a modern fixed-target experiment with a record energy and with high luminosities can help answer problems at the frontier of particle and astroparticle physics. We divide this section into three parts for which the physics cases are quite distinct. In the first part, we discuss the impact of such an experiment on our understanding of the high- $x$ structure of nucleons. In the second part, the physics case for the high- $x$ structure of complex nuclei is considered. Finally, the third part is devoted to astroparticle-physics applications.

\subsubsection{Nucleon structure}

Much progress has been made in the past 30 years in our understanding of the partonic structure of nucleons. The parton distribution functions (PDFs) are determined in global analyses [174-179] using a wealth of experimental information from fixed-target and collider experiments. The analyses are routinely performed at next-to-leading order (NLO) and next-to-next-to-leading order (NNLO) of perturbative QCD and the uncertainties of the PDFs are carefully evaluated. Still, at high momentum fractions $x$, the PDFs are poorly known, in particular the smaller distributions. For example, the uncertainty of the gluon distribution gets very large at $x \gtrsim 0.4$ and the strange, charm and bottom PDFs are completely unconstrained in this kinematic range. A better understanding of the high- $x$ structure of the nucleon is warranted for several reasons:

- First, while it is well known that the gluon carries over $40 \%$ of the nucleon momentum, most of the gluons carry a small momentum fraction. On the other hand, in a constituent quark picture, it is rather the gluon distribution carrying a high momentum fraction which can be interpreted as binding the constituent quarks together. Furthermore, light-cone models predict a relatively sizeable high- $x$ component of the strange, charm and bottom PDFs. This means that the higher the probed $x$-values will be, the better these fundamental aspects of QCD can be studied. Needless to say, that progress on the high- $x$ gluon, strange and charm PDFs will lead to a refined picture for the light-quark valence and sea distributions in this kinematic region.

- At the same time, PDFs are a crucial input for making theoretical predictions for observables at the LHC. In many cases, the PDF uncertainty has become the limiting factor in the accuracy of the predictions. This is particularly true for processes involving heavy new states in Beyond the Standard Model (BSM) theories where the high- $x$ PDFs are probed. Clearly, an improved understanding of the high- $x$ PDFs is crucial for BSM searches at the LHC and any future hadron collider, and AFTER@LHC offers the unique opportunity to study these aspects of high- $x$ hadron structure in detail.

Kinematic coverage of lepton pair production. As is well known, at leading order (LO) the cross section for DY lepton-pair production in a collision of two nucleons $A$ and $B$ is given by the following expression:

$$
\frac{d^{2} \sigma}{d x_{1} d x_{2}}=\frac{4 \pi \alpha^{2}}{9 s x_{1} x_{2}} \sum_{i} e_{i}^{2}\left[q_{i}^{A}\left(x_{1}\right) \bar{q}_{i}^{B}\left(x_{2}\right)+\bar{q}_{i}^{A}\left(x_{1}\right) q_{i}^{B}\left(x_{2}\right)\right],
$$

where $e_{i}$ is the electric charge of the quark (in units of $e$ ) and the sum runs over all active quark flavours. Therefore, it is clear that this process provides information on the (light) quark sea. Existing DY data which are used in global PDF analyses come from fixed-target experiments at Fermilab (E866/NuSea, E605) and the LHC. 


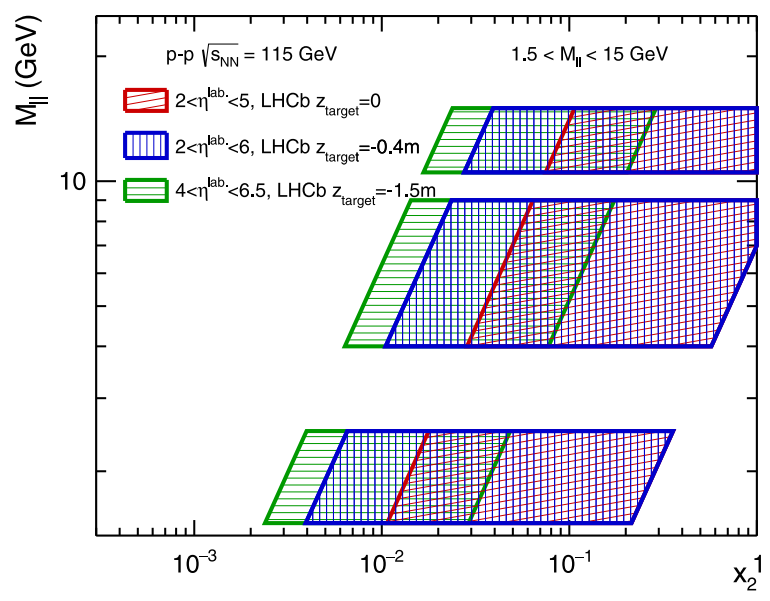

(a)

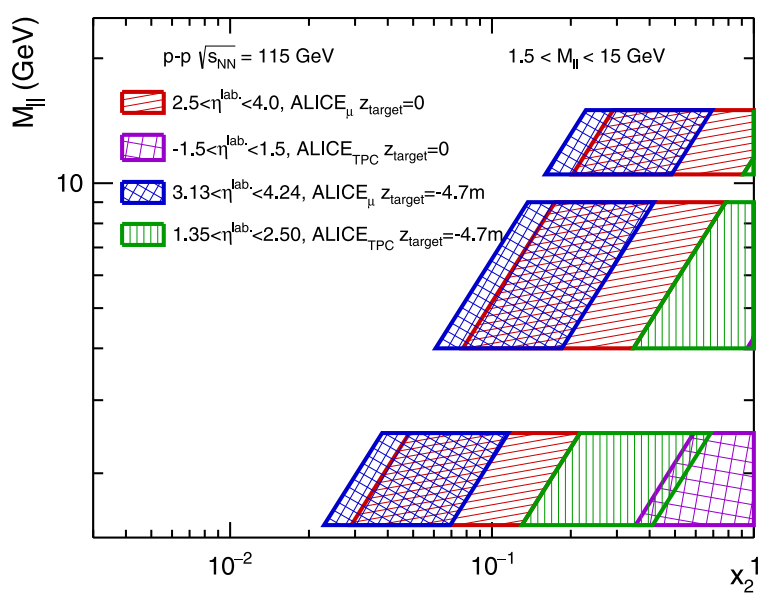

(b)

Fig. 14. Simplified kinematical reach in $x_{2}$ for DY lepton-pair production with AFTER@LHCb and AFTER@ALICE in $p p$ collisions at $\sqrt{s}=115$ GeV for different muon-pair acceptances and invariant masses. The acceptances in $\eta_{(\ell \ell}^{\text {lab }}$ correspond to different target locations within (a) LHCb and (b) ALICE. [No $p_{T, \mu}$ constraint is applied.]

The cross section is usually given as a function of the invariant mass of the lepton pair $M_{(\ell \ell)}$ (at LO $M^{2}=x_{1} x_{2} s$ ) and the Feynman variable $x_{F}=x_{1}-x_{2}$ from which the momentum fractions $x_{1,2}$ can approximately be recovered using the relation $x_{1,2}=\left(\sqrt{x_{F}^{2}+4 \tau} \pm x_{F}\right) / 2$ where $\tau=M_{\ell \ell}^{2} / s$. Correspondingly, one has in terms of the pair rapidity in the c.m.s., $Y_{\ell \ell}^{\text {c.m.s. }}, x_{1,2}=M_{\ell \ell} / \sqrt{s} e^{ \pm Y_{\ell \ell}^{\text {c.m.s. }} \text {. }}$.

Fig. 14 shows a simplified kinematical reach with AFTER@LHCb and AFTER@ALICE for DY measurements at $\sqrt{s}=$ $115 \mathrm{GeV}$ considering different (pseudo)rapidity domains ${ }^{19}$ which follow from the acceptance of the specified detectors viewed from the different indicated target locations. In the LHCb case, a target located at 0 or $-0.4 \mathrm{~m}$ offers similar reaches whereas at $-1.5 \mathrm{~m}$ the reach is clearly shifted to lower $x$. In particular, even above the bottomonium region where the statistics will be limited, one does not access $x_{2}=1$ any more. In the ALICE case, even with a remote target, e.g. at $-4.7 \mathrm{~m}$, one can still reach $x_{2}=1$ between the charmonium and bottomonium family. The Intermediate Mass Region (IMR) below the charmonium family, which de facto offers the largest statistics, still covers the valence region in the case of the ALICE CB. Projection studies of the DY yield in this region, which is complementary to the DIS studies at JLab, would however essentially rely on the technique used to subtract the combinatorial background. As such, they would be driven rather by systematical uncertainty projections than by statistical ones. In this context, we will limit ourselves to discuss DY projections above the charmonium family, namely for $M_{\ell \ell}>4 \mathrm{GeV}$. However, this does not mean at all that IMR DY studies are not possible with AFTER@LHCb and AFTER@ALICE.

In Fig. 15, the kinematical acceptance for DY lepton-pair production is shown assuming $p p$ collisions at a c.m.s. energy of $\sqrt{s}=115 \mathrm{GeV}$ with an integrated luminosity of $10 \mathrm{fb}^{-1}$ and a single-muon acceptance of $2<\eta_{\mu}^{\text {lab. }}<5$ and $p_{T, \mu}>$ $1.2 \mathrm{GeV}$. It should be noted that each cell contains at least 30 events. For comparison, the kinematic coverage of existing DY data (E605, E866/NuSea) used in global proton PDF analyses is depicted. ${ }^{20}$ The NuSea data have been obtained in $800 \mathrm{GeV} p p$ and $p d$ collisions $(\sqrt{s}=38.8 \mathrm{GeV})$ covering the di-muon mass ranges from 4.2 to $8.7 \mathrm{GeV}$ and 10.85 to $16.85 \mathrm{GeV}$ and the Feynman- $x_{F}$ range from -0.05 to 0.8 . As anticipated with our simplified kinematical-reach analysis, AFTER@LHC will be able to extend the coverage up to even higher $x$-values close to one. Furthermore, while the NuSea data are dominated by statistical uncertainties reaching 100\% at the kinematic boundaries, AFTER@LHC will considerably improve the precision due to the higher centre-of-mass energy and the higher luminosity.

The DY measurements at AFTER@LHC provide important tests of nucleon structure. In the limit $x_{F} \rightarrow-1$ and moderate or small invariant masses $M_{\ell \ell}$, we have $x_{1} \simeq M_{\ell \ell}^{2} /\left(s\left|x_{F}\right|\right), x_{2} \gtrsim\left|x_{F}\right|$. For example, for $x_{F}=-0.8, M_{\ell \ell}=10\left(M_{\ell \ell}=15\right) \mathrm{GeV}$ we have $x_{2} \simeq 0.8$ and $x_{1} \simeq 0.01\left(x_{1} \simeq 0.02\right)$. In this kinematic region the ratio of the DY cross section in pn collisions with the one in $p p$ collisions is approximately given by (LO, $\bar{u}\left(x_{2}\right), \bar{d}\left(x_{2}\right), s\left(x_{2}\right), \bar{s}\left(x_{2}\right) \ll u_{v}\left(x_{2}\right), d_{v}\left(x_{2}\right)$, neglecting $Z$-exchange):

$$
R=\frac{\sigma^{\mathrm{DY}}(p n)}{\sigma^{\mathrm{DY}}(p p)} \simeq \frac{4 \bar{u}\left(x_{1}\right) d\left(x_{2}\right)+\bar{d}\left(x_{1}\right) u\left(x_{2}\right)}{4 \bar{u}\left(x_{1}\right) u\left(x_{2}\right)+\bar{d}\left(x_{1}\right) d\left(x_{2}\right)} \simeq \frac{4 d\left(x_{2}\right)+u\left(x_{2}\right)}{4 u\left(x_{2}\right)+d\left(x_{2}\right)}=\frac{1+4 r_{v}}{4+r_{v}}
$$

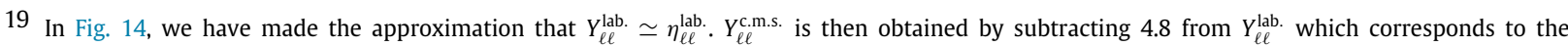
rapidity shift between the lab. and c.m.s. frames with a $7 \mathrm{TeV}$ beam.

20 We are grateful to V. Bertone from the NNPDF collaboration for providing us the points.
} 


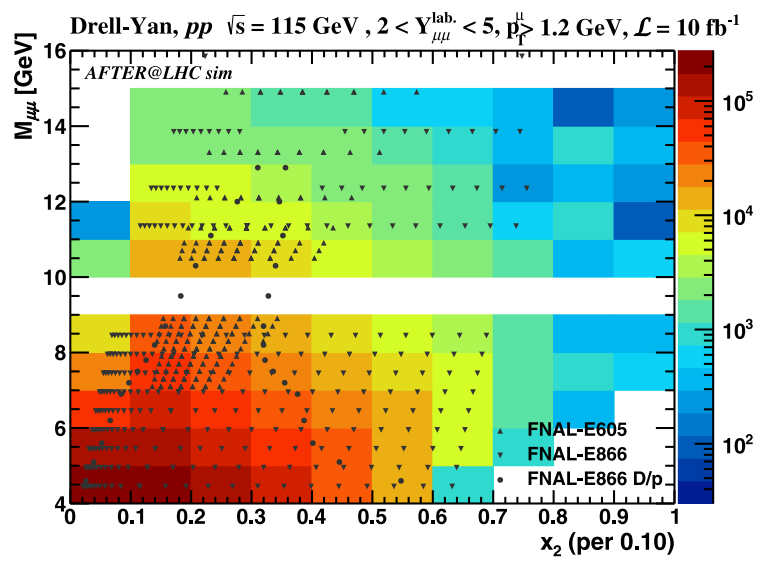

(a)

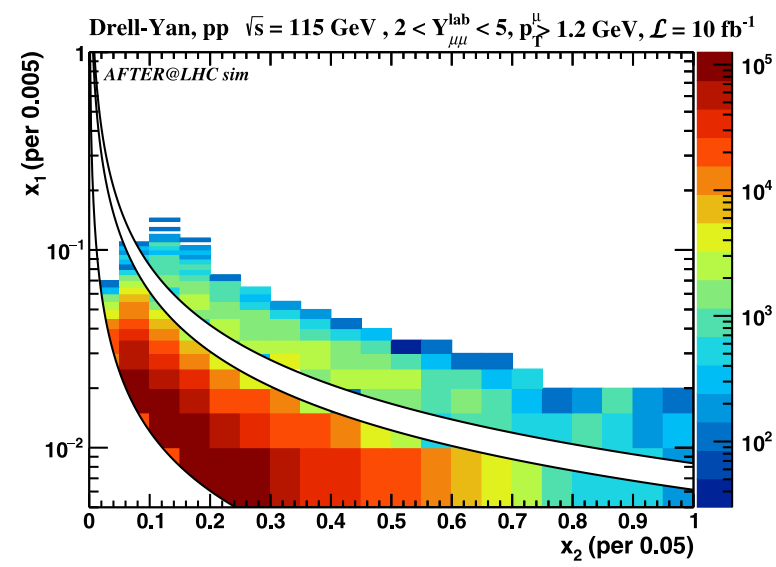

(b)

Fig. 15. Kinematical acceptance for DY lepton-pair production with AFTER@LHCb in pp collisions at $\sqrt{s}=115$ GeV with a muon-pair acceptance of $2<Y_{\mu \mu}^{\text {lab. }}<5$ and single-muon requirements: $2<\eta_{\mu}^{\text {lab }}<5$ and $p_{T}^{\mu}>1.2 \mathrm{GeV}$. (a) Di-muon invariant mass vs. $x_{2}$ compared to the existing DY data [180-183] used in current global PDF fits. (b) $x_{1}$ vs $x_{2}$ for the considered di-muon invariant-mass range of 4 GeV $<M_{\mu \mu}<15$ GeV excluding $\Upsilon$ mass range of $9 \mathrm{GeV}<M_{\mu \mu}<10.5 \mathrm{GeV}$. Colours correspond to expected yields of the DY signal in each kinematical region, and each coloured cell contains at least 30 events. We stress that the bins with a number of counts less that 30 are not shown in these AFTER@LHCb projections, whereas some of the shown data points from the FNAL experiments include bins with significantly less counts.

where $\bar{d}\left(x_{1}\right) \simeq \bar{u}\left(x_{1}\right)$ has been used to arrive at the third equality and $r_{v}=d\left(x_{2}\right) / u\left(x_{2}\right) \simeq d_{v}\left(x_{2}\right) / u_{v}\left(x_{2}\right)$. Interestingly, exactly the same LO parton model expression is found for the ratio of structure functions $F_{2}^{n}\left(x, Q^{2}\right) / F_{2}^{p}\left(x, Q^{2}\right)$ in the limit $x \rightarrow 1$ which corresponds to elastic scattering. As a further consequence, the ratio $R$ is bounded, $1 / 4 \leq R \leq 4$, similarly to the famous bounds for the ratio of DIS functions, $1 / 4 \leq F_{2}^{n} / F_{2}^{p} \leq 4$, derived by Nachtmann [184]. The PDFs vanish for $x \rightarrow 1$ and generally, the high- $x$ behaviour of the PDFs at the initial scale $Q_{0}$ is parametrised as $x f_{i}\left(x, Q_{0}\right) \propto(1-x)^{b_{i}}$ where $b_{i}$ depends on the parton flavour " $i$ ". Currently, only $b_{u_{v}}$ is relatively well constrained with values in the range $2.6 \lesssim b_{u_{v}} \lesssim 3.6$, which is in agreement with the expectation from counting rules [185] $\left(b_{u_{v}}=b_{d_{v}}=3\right)$, whereas $b_{d_{v}}$ is known to a much lesser extent and varies strongly between 1.4 and 4.6 for different sets of PDFs, see Figs. 2 and 5 in [186]. ${ }^{21}$ Note also that the CJ15 analysis [187] which has a particular focus on the high- $x$ region points to a constant $d(x) / u(x) \sim 0.1$ for $x \rightarrow 1$ implying $R \rightarrow \sim 0.34$. However, for the time being it is reasonable to allow for the possibilities that $r_{v}$ can vanish, approach a finite value $k$, or diverge in the limit $x \rightarrow 1$ (see Fig. 8 in [186]). Consequently, we find in the limit $x_{2} \rightarrow 1$ that a measurement of $R$ could constrain $r_{v}$ and provide important tests of different models of nucleon structure [188-193].

Experimentally, it is the ratio of cross sections in $p d$ over $p p$ collisions which is accessible. Neglecting any nuclear effects in deuterium the ratio can be written as

$$
R_{d / p}\left(x_{2}\right)=\frac{\sigma^{\mathrm{DY}}(p d)}{\sigma^{\mathrm{DY}}(p p)}=1+\frac{\sigma^{\mathrm{DY}}(p n)}{\sigma^{\mathrm{DY}}(p p)} \simeq 5 \frac{1+r_{v}\left(x_{2}\right)}{4+r_{v}\left(x_{2}\right)} .
$$

Consequently, we find in the limit $x_{2} \rightarrow 1$

$$
R_{d / p} \rightarrow\left\{\begin{array}{lll}
2 & ; & r_{v}=1 \\
2.5 & ; & r_{v}=0 \\
5 & ; & r_{v} \rightarrow \infty
\end{array},\right.
$$

and a sufficiently precise measurement of the ratio will allow one to determine $r_{v}(x)=d(x) / u(x)$ at high- $x$. In practice, a full fledged QCD analysis at NLO or NNLO of the data needs to be performed.

Drell-Yan lepton-pair production and PDFs. In order to estimate the possible impact of the DY lepton-pair production in $p p$ collisions at AFTER@LHC on the PDFs, we have performed a profiling analysis [194] using the xFitter package [195]. For this purpose we have used pseudo-data constructed out of NLO QCD predictions for the rapidity distributions in the c.m.s. using the MCFM programme [196] and projected experimental uncertainties adding the statistical uncertainty from the DY yield and the uncertainties from the subtraction of the combinatorial background in quadrature. The statistical uncertainties were estimated assuming a yearly integrated luminosity of $\mathcal{L}_{p p}=10 \mathrm{fb}^{-1}$. No additional systematic uncertainty has been taken into account. The pseudo-data have been generated for several bins in the invariant mass of the muon pair

21 Needless to say that the exponents for the gluon and the quark sea are very poorly known. 


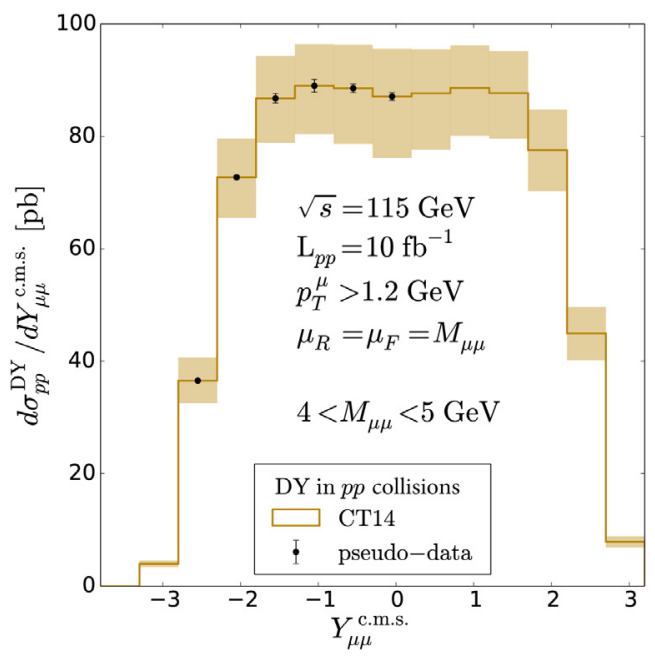

Fig. 16. DY lepton-pair-production cross section in $p p$ collisions as a function of the muon-pair c.m.s. rapidity for the invariant mass $4 \mathrm{GeV}<M_{\mu \mu}<$ $5 \mathrm{GeV}$, with $p_{T}^{\mu}>1.2 \mathrm{GeV}$. The NLO theory predictions obtained using CT14 PDFs are overlaid by pseudo-data.

$\left(M_{\mu \mu} \in[4,5],[5,6],[6,7],[7,8] \mathrm{GeV}\right.$ and $\left.M_{\mu \mu}>10.5 \mathrm{GeV}\right)$ and have been constructed such that the central values of the "measurements" and predictions coincide. This is illustrated in Fig. 16 for the invariant-mass bin $4<M_{\mu \mu}<5 \mathrm{GeV}$. In some cases the uncertainties are smaller than the data points and therefore not visible. ${ }^{22}$ As can be seen, the band showing the uncertainty of the theory prediction due to the NLO CT14 PDF uncertainties is much larger than errors of the simulated data.

The effect of the profiling analysis, showing the decrease of the PDF uncertainties after including these data in a PDF global fit, is presented in Figs. 17 (logarithmic in $x$ ) and 18 (linear in $x$ ) for the light-quark $(f=u, d, \bar{u}, \bar{d}$ ) distributions. To be precise, for each of these PDFs the upper and lower curves delimiting the bands are defined as

$$
R_{f}(x, Q)=1 \pm \frac{1}{2 f_{0}(x, Q)} \sqrt{\sum_{i}\left[f_{i+}(x, Q)-f_{i-}(x, Q)\right]^{2}},
$$

where $f_{0}(x, Q)$ is the central PDF and $f_{i \pm}(x, Q)$ are the 'i-th' error PDF in the plus or minus direction and a sum over all eigenvector directions is performed. Remarkably, Fig. 17 shows a sizeable reduction of the PDF uncertainties not only in the high- $x$ but also in the intermediate and small $x$ region $\left(x \sim 0.1 \ldots 10^{-4}\right)$. The effect is largest for the $u$ and $\bar{u}$ distributions but it is also substantial for the $d$ and $\bar{d}$ PDFs. The main focus of this section is the high- $x$ region which is highlighted in Fig. 18. Here it can be seen that our knowledge of the valence quark distributions can be considerably improved for $x \gtrsim 0.4$ where the effect is again more pronounced for the up quark. However, even some information on the light quark sea at high- $x$ can be obtained.

Additionally, we have considered a scenario with luminosity reduced by a factor 10 , i.e. $\mathcal{L}_{p p}=1 \mathrm{fb}^{-1}$. From Figs. 17 and 18, it is clear that even such a reduced statistics will allow for a substantial reduction of the current PDF uncertainties.

$W$ boson production close to the threshold. Due to the high c.m.s. energy of $115 \mathrm{GeV}$, it is possible to study the production of $W$ bosons close to the production threshold. ${ }^{23}$ Assuming a yearly integrated luminosity of $10 \mathrm{fb}^{-1}$, we expect roughly $250 \mathrm{~W}^{+}$and $60 \mathrm{~W}^{-}$events per year before taking into account the experimental efficiencies. ${ }^{24}$ These event numbers are based on NNLO cross sections calculated by integrating over the pseudo-rapidity range $2<\eta_{\ell}^{\text {lab. }}<5$ and imposing a requirement of $p_{T}^{\ell}>10 \mathrm{GeV}$ on the transverse momentum on the $W$-decay lepton using FEWZ [197] together with NNLO CT14 PDFs [5]. The factorisation and renormalisation scales have been chosen to be $\mu_{R}=\mu_{F}=M_{W}$. For convenience, the cross sections at NLO and NNLO along with the event numbers and the PDF uncertainties are summarised in Table 13 for a selection of $p_{T}^{\ell}$ kinematic cuts.

In Fig. 19, we show NNLO predictions for the differential cross section for $W^{+}$production in $p p$ collisions at AFTER@LHC as a function of the transverse mass $M_{T}$ for the case of $p_{T}^{\ell}>10 \mathrm{GeV}$ (left) and the transverse momentum $p_{T}^{\ell}$ of the produced lepton (right). The yellow band represents the PDF uncertainty and the error bars represent the uncertainty

\footnotetext{
22 Note that the covered rapidity range in the c.m.s. is from $-2.8<y_{\text {c.m.s. }}<0.2$ such that there are no generated data at rapidities $y_{\text {c.m.s. }}>0.2$.

23 Note that the cross section for $Z$ boson production is too low to be accessible at AFTER@LHC.

24 These numbers are for one leptonic decay channel. In a more realistic estimate, it will be necessary to sum up the electron and muon channels taking into account the different efficiencies.
} 


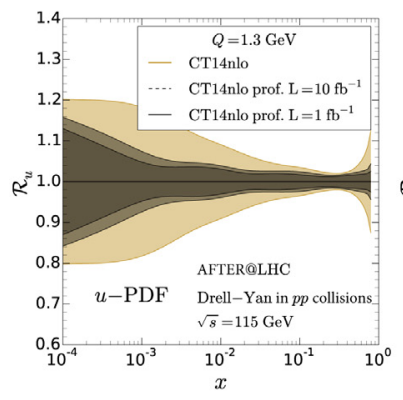

(a) $u \mathrm{PDF}$

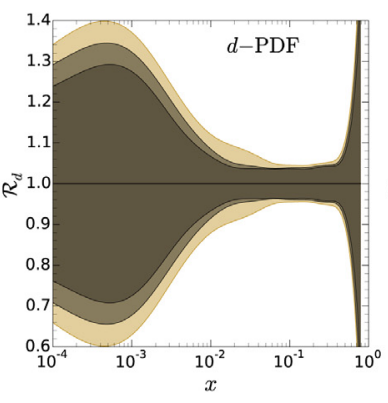

(b) $d \mathrm{PDF}$

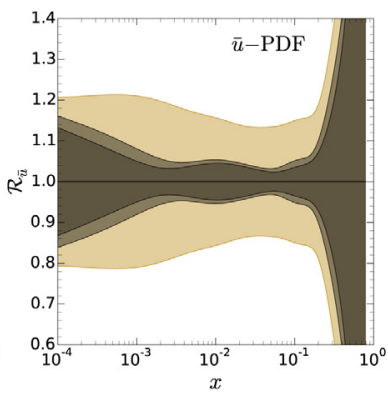

(c) $\bar{u} \mathrm{PDF}$

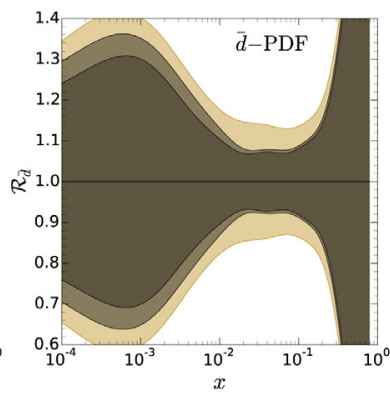

(d) $\bar{d}$ PDF

Fig. 17. Impact of the DY lepton-pair production in pp collisions at $\sqrt{s}=115 \mathrm{GeV}$ on the PDF uncertainties. The $u, d, \bar{u}$ and $\bar{d}$ PDFs from CT14 [5] are plotted as a function of $x$ at a scale $Q=1.3 \mathrm{GeV}$ before and after including AFTER@LHCb pseudo-data in the global analysis using the profiling method [194,195]. Two scenarios with different integrated luminosities were considered: inner band: $\mathcal{L}_{p p}=10 \mathrm{fb}^{-1}$, middle band: $\mathcal{L}_{p p}=1 \mathrm{fb}^{-1}$ (the outer band represents current PDF uncertainties).

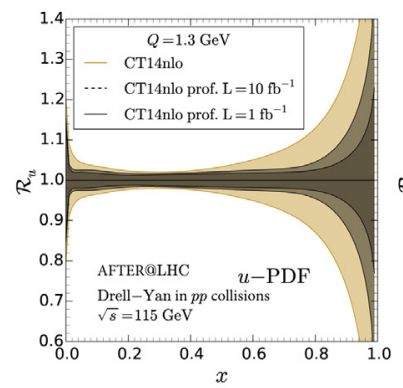

(a) $u$ PDF

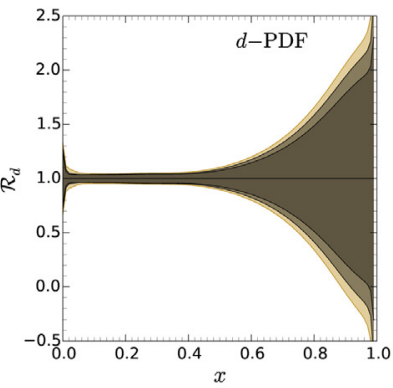

(b) $d$ PDF

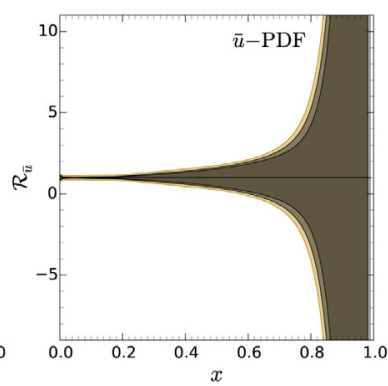

(c) $\bar{u}$ PDF

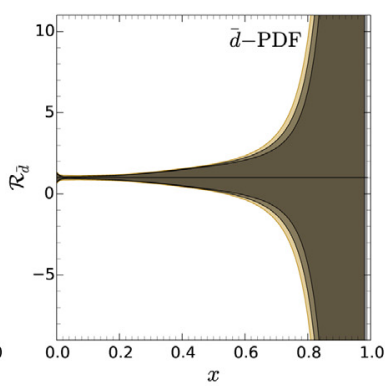

(d) $\bar{d} \mathrm{PDF}$

Fig. 18. Same as in Fig. 17 on a linear scale highlighting the high- $x$ region.

\section{Table 13}

Cross sections in [fb] at NLO and NNLO integrated over the pseudo-rapidity range $2<\eta_{\mu}^{\text {lab. }}<5$ and imposing a cut $p_{T}^{\mu}>10 \mathrm{GeV}$. The results have been obtained for $p p$ collisions at $\sqrt{s}=115 \mathrm{GeV}$ with FEWZ [197] using the NLO and NNLO CT14 PDFs [5], respectively. The renormalisation and factorisation scales have been set to $\mu_{R}=\mu_{F}=M_{W}$. The asymmetric uncertainties have been calculated using the error PDFs. The expected number of events has been obtained with a yearly luminosity of $10 \mathrm{fb}^{-1}$.

\begin{tabular}{llllllll}
\hline$p p$ & $W^{+}$ & & & & \\
\cline { 2 - 3 } & NLO & NNLO & Counts/year & & NLO & NNLO & Counts/year \\
\hline$p_{T}^{l}>10 \mathrm{GeV}$ & $22.5_{-4.3}^{+4.8}$ & $25.9_{-5.0}^{+4.8}$ & $259 \pm 49$ & $5.5_{-1.3}^{+1.3}$ & $6.2_{-1.4}^{+1.1}$ & $62 \pm 13$ \\
\hline$p_{T}^{l}>20 \mathrm{GeV}$ & $1.9_{-0.7}^{+1.2}$ & $2.3_{-1.1}^{+1.3}$ & $23 \pm 12$ & $0.38_{-0.20}^{+0.29}$ & $0.50_{-0.25}^{+0.25}$ & $5 \pm 2.5$ \\
\hline$p_{T}^{l}>30 \mathrm{GeV}$ & $0.28_{-0.27}^{+0.91}$ & $0.27_{-0.24}^{+0.72}$ & $2.7 \pm 4.8$ & $0.035_{-0.039}^{+0.091}$ & $0.04_{-0.04}^{+0.09}$ & $0.4 \pm 0.7$ \\
\hline
\end{tabular}

due to renormalisation/factorisation scale variation by a factor 2 around the central scale choice $\mu_{R}=\mu_{F}=M_{W}$. As can be seen, the PDF uncertainty dominates over the scale uncertainty for $M_{T}>20 \mathrm{GeV}$. It is also interesting to note that the $M_{T}$ distribution peaks at $M_{T} \sim 25 \mathrm{GeV}$ far below $M_{W}$.

In the following, we illustrate that even a rough measurement of the $W$ cross section at AFTER can provide interesting information on the high- $x$ behaviour of the light sea quarks. The LO cross section for $W$ boson production reads

$$
\frac{d \sigma}{d y}=\frac{2 \pi}{3} \frac{G_{F}}{\sqrt{2}} \sum_{i, j}\left|V_{i j}\right|^{2}\left[q_{i}^{A}\left(x_{1}\right) \bar{q}_{j}^{B}\left(x_{2}\right)+\bar{q}_{j}^{A}\left(x_{1}\right) q_{i}^{B}\left(x_{2}\right)\right],
$$

where the momentum fractions $x_{1,2}$ are related to the (c.m.s.) rapidity of the $W$ boson in the usual way, $x_{1,2}=$ $\left(M_{W} / \sqrt{s}\right) e^{ \pm y}$. Assuming a diagonal CKM matrix and neglecting the contribution from the sc-channel one can easily derive 


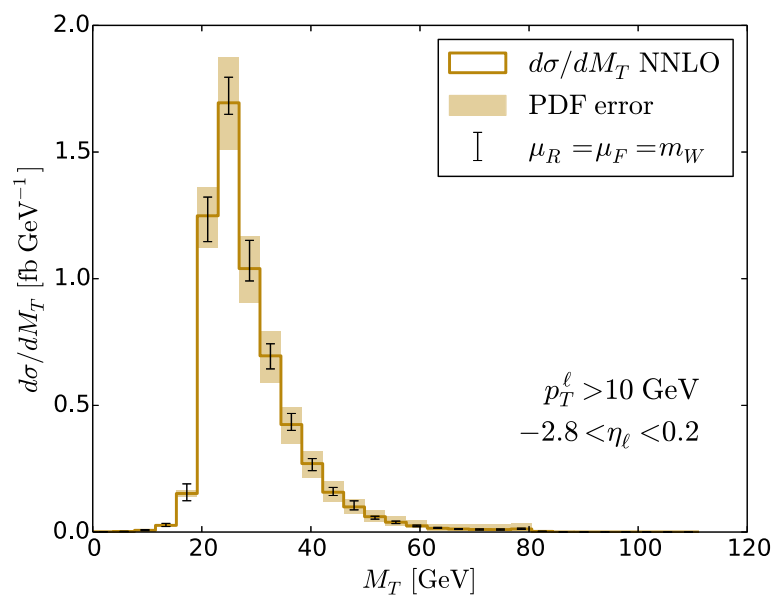

(a)

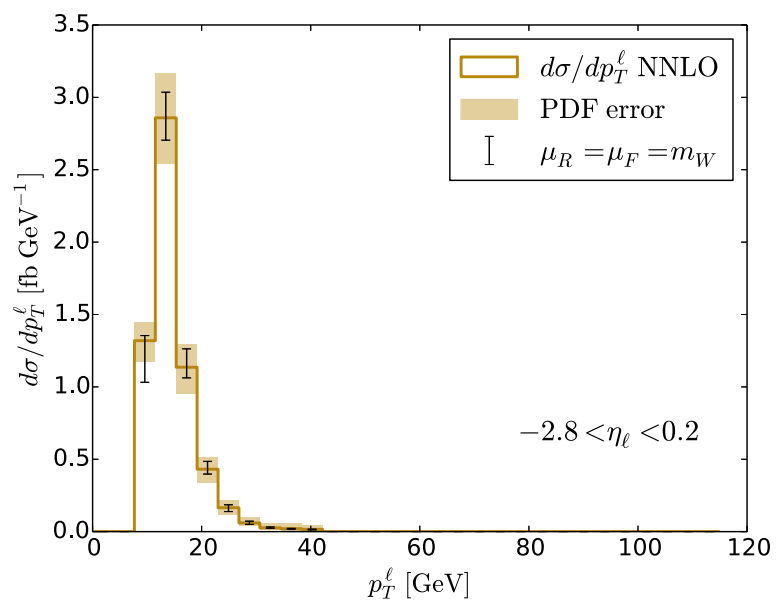

(b)

Fig. 19. NNLO cross section in $[\mathrm{fb} / \mathrm{GeV}]$ for $W^{+}$production in $p p$ collisions at AFTER@LHC as a function of (a) the transverse mass $M_{T}$ and (b) the transverse momentum $p_{T}^{\ell}$ of the produced lepton. The cross section has been obtained by integrating over the pseudo-rapidity range $2<\eta_{\ell}<5$ (in the laboratory frame) using FEWZ [197]. For the $M_{T}$-distribution (left) $p_{T}^{\ell}>10 \mathrm{GeV}$ has been imposed. The yellow bands represent the PDF uncertainty and the error bars represent the uncertainty due to renormalisation/factorisation scale variation by a factor 2 around the central scale choice $\mu_{R}=\mu_{F}=M_{W}$.

the following ratio of cross sections:

$$
\begin{aligned}
R^{W} & =\frac{\frac{d \sigma}{d y}\left(p n \rightarrow W^{+}+W^{-}\right)-\frac{d \sigma}{d y}\left(p p \rightarrow W^{+}+W^{-}\right)}{\frac{d \sigma}{d y}\left(p n \rightarrow W^{+}+W^{-}\right)+\frac{d \sigma}{d y}\left(p p \rightarrow W^{+}+W^{-}\right)}=1-2 \frac{\frac{d \sigma}{d y}\left(p p \rightarrow W^{+}+W^{-}\right)}{\frac{d \sigma}{d y}\left(p d \rightarrow W^{+}+W^{-}\right)} \\
& =\frac{\left[u\left(x_{1}\right)-d\left(x_{1}\right)\right]\left[\bar{u}\left(x_{2}\right)-\bar{d}\left(x_{2}\right)\right]+\left[\bar{u}\left(x_{1}\right)-\bar{d}\left(x_{1}\right)\right]\left[u\left(x_{2}\right)-d\left(x_{2}\right)\right]}{\left[u\left(x_{1}\right)+d\left(x_{1}\right)\right]\left[\bar{u}\left(x_{2}\right)+\bar{d}\left(x_{2}\right)\right]+\left[\bar{u}\left(x_{1}\right)+\bar{d}\left(x_{1}\right)\right]\left[u\left(x_{2}\right)+d\left(x_{2}\right)\right]} .
\end{aligned}
$$

At central rapidity, $x_{1}=x_{2}=x$, the ratio reduces to the remarkably simple expression ${ }^{25}$

$$
R^{W}\left(y_{\text {c.m.s. }}=0\right)=\frac{\left(1-r_{v}\right)\left(1-r_{s}\right)}{\left(1+r_{v}\right)\left(1+r_{s}\right)}
$$

where $r_{v}(x)=d(x) / u(x)$ and $r_{s}(x)=\bar{d}(x) / \bar{u}(x)$ at $x \sim 0.3$. Therefore, even a rough measurement of this ratio with about $30 \%$ precision could provide valuable information on the barely known ratio $r_{s}=\bar{d} / \bar{u}$ at high $x$. This would already compete with the existing E866 measurements [183] and 10\% precision would be welcome, which could be obtained by accumulating $W$ data over a couple of years. On the other hand, we estimate that such a measurement will not be able to compete with the possible future SeaQuest DY measurements [198].

Another interesting aspect is that a measurement of vector-boson production close to the threshold could serve as a proxy for searches of new heavy resonances at the LHC. As was highlighted above the $W$-boson production at AFTER@LHC is predominantly off-shell. Therefore, one can expect a similar behaviour for a heavy new resonance with a mass close to the c.m.s. energy. The current mass limits for such heavy resonances are typically on the order of 3 to $4 \mathrm{TeV}$ depending on the model. With increasing statistics even higher resonance masses will be probed, and we are approaching the region of the production threshold where the high- $x$ PDFs are probed and the PDF error becomes the dominant theoretical uncertainty in precision calculations $[199,200]$. Furthermore, soft gluon resummation effects are expected to become important [199] which could be partially tested at AFTER@LHC.

The charm quark PDF at high $x$. The high- $x$ heavy-quark PDFs can be important for BSM physics in which new heavy particles have couplings to the SM fermions which are proportional to the fermion mass or for models which predominantly couple to the second and/or third generation [201]. Most global analyses of PDFs rely on the assumption that the charm and bottom PDFs are perturbatively generated by gluon splitting, $g \rightarrow Q \bar{Q}$, and do not involve any nonperturbative degrees of freedom. It is clearly necessary to test this hypothesis with suitable QCD processes. Conversely, a non-perturbative, intrinsic contribution to the heavy-quark PDF in the proton comes from QCD diagrams in which the

25 At central rapidity in the c.m.s., $y=0$, one has $x_{1}=x_{2}=M_{W} / \sqrt{s}$. However, as shown in Fig. 19, most $W$ bosons are produced off-shell. In that case one can effectively replace $x_{1}=x_{2} \sim M^{*} / \sqrt{s}$ with $M^{*} \sim 35 \mathrm{GeV}$. 


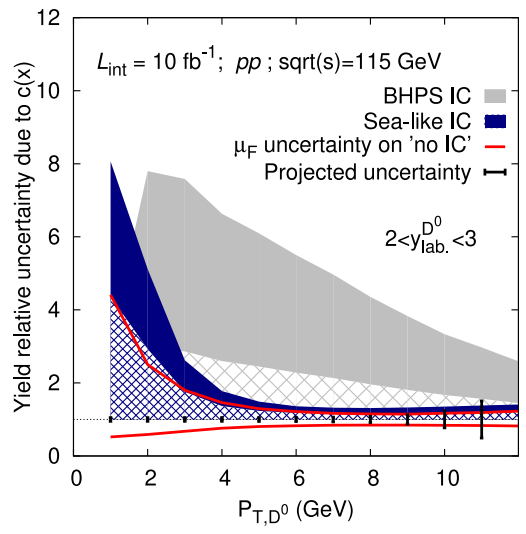

(a) $2<y_{\text {lab. }}<3$

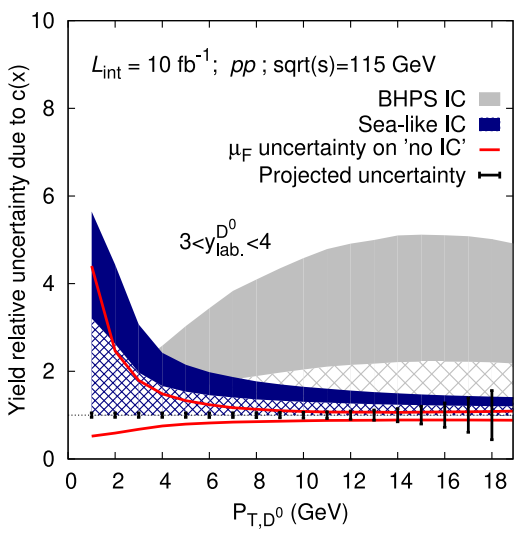

(b) $3<y_{\text {lab. }}<4$

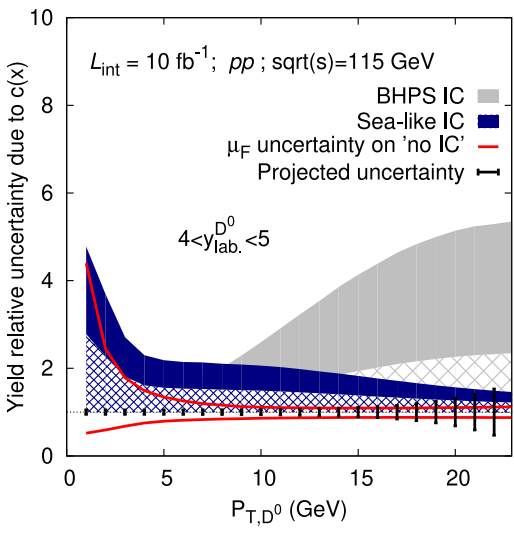

(c) $4<y_{\text {lab. }}<5$

Fig. 20. Impact of the uncertainty on the charm content of the proton on the $D^{0}$ yield as a function of $p_{T}$ compared to projected uncertainties from the measurement of the $D^{0}$ yield in $p p$ collisions at $\sqrt{s}=115 \mathrm{GeV}$ in the LHCb acceptance. The grey (resp. blue) zones correspond to yields computed with charm PDFs including BHPS-like (resp. sea-like) IC [217,218]. The filled areas correspond to yields computed with up to $\left\langle x_{c+\bar{c}}\right\rangle=2 \%($ resp. $2.4 \%)$ and the hashed areas up to $\left\langle x_{c+\bar{c}}\right\rangle=0.57 \%$ (resp. 1.1\%). The dashed red curves indicate the factorisation scale $\left(\mu_{F}\right)$ uncertainty on the 'no-IC' yield obtained by varying $\mu_{\mathrm{F}}$ between $m_{T}$ and $2 m_{T}$ with $m_{T}^{2}=m_{\mathrm{c}}^{2}+p_{T}^{2}$. Systematic uncertainties of $5 \%$ are included and the statistical uncertainty for the background subtraction is assumed to be negligible which is reasonable assuming LHCb-like performances, see [117]. The rates were computed by assuming an average efficiency of $\langle\varepsilon\rangle=10 \%$ and $\mathcal{B}\left(D^{0} \rightarrow K \pi\right)=3.93 \%$.

heavy-quark pair is attached by two or more gluons to the valence quarks. It thus depends on the non-perturbative intrinsic structure of the proton [202-204]. For a recent review, see [205].

There are extensive indications for charm production at high $x$, which are, however, not yet fully conclusive and new data from the LHC, a future EIC and a fixed-target experiment like AFTER@LHC will be necessary. One example is the EMC measurement of $c\left(x, Q^{2}\right)$ in muon DIS [206]. The rate observed by the EMC was found to be approximately 30 times higher at $x=0.42, Q^{2}=75 \mathrm{GeV}^{2}$ than predicted by gluon splitting [207]. In a more recent analysis, the EMC data have been described in the context of a fitted charm quark distribution which increases the stability of the fit with respect to variations of the charm quark mass [208]. In this study, the NNPDF collaboration found a fitted high- $x$ component peaking at $x \sim 0.5$ and carrying about $1 \%$ of the total proton momentum. At the same time, they observed that the EMC data cannot be fitted with a perturbatively generated charm PDF and the additional parameters, effectively parametrising the high- $x$ intrinsic component, are needed to describe these data. Therefore, there is already some evidence for a high- $x$ intrinsic charm (IC) component carrying about $1 \%$ of the total momentum of the proton. ${ }^{26}$ IC also predicts the observed features of the ISR data for $\frac{d \sigma}{d x_{F}}\left(p p \rightarrow \Lambda_{c} X\right)$ [209] and more recently by SELEX [210]. In this process, the comoving $c, u$ and $d$ coalesce to produce the $\Lambda_{c}$ at high $x_{F}$, where $x_{F}=x_{c}+x_{u}+x_{d}$. Other observations at high $x_{F}$ include $\Lambda_{b}, \Lambda_{c}$, single and double quarkonium, double-charm baryons $c c u$, $c c d$, we refer to [205] for a more complete overview. We also note here recent works [211-213] providing predictions for doubly heavy baryons, $B_{c}$ mesons and all-charmed tetra quarks at AFTER@LHC based on the intrinsic heavy-quark mechanism, showing that this mechanism should dominate at high $x_{F}$ allowing for its observation at AFTER@LHC and potentially confirming the existence of the IC. $\Xi_{c c}$ production at AFTER@LHC was also studied from DGLAP-generated charm in [214]. Besides, the production of the hidden charm pentaquark $P_{c}^{+}$was found to peak, for the AFTER@LHC kinematics, at about $y_{\mathrm{lab} .} \simeq 1.4$ [215] using the same formalism as that of a previous study for the LHC kinematics [216].

A non-perturbative IC component modifies the predictions for a number of processes at the LHC such as inclusive $D$ meson production $[219,220]$ or the associated production of a heavy quark with a photon [221] or $Z+c$ production [208]. However, in these examples, one has to go to relatively large transverse momenta or to very forward rapidities to expect a sizeable effect.

Simply owing to the large boost between the laboratory frame and the c.m.s., the fixed-target mode is the ideal set-up to uncover an excess of charm at high $x$. To illustrate this statement, we show, in Fig. 20, the relative yield uncertainty for inclusive $D^{0}$ meson production at AFTER@LHC for three rapidity bins $\left(2<y_{\text {lab. }}<3,3<y_{\text {lab. }}<4,4<y_{\text {lab. }}<5\right)$ as a function of the transverse momentum $\left(P_{T, D^{0}}\right)$ of the $D^{0}$ meson. An integrated luminosity of $10 \mathrm{fb}^{-1}$ has been assumed to compute the expected yields - accounting for the expected efficiency and the branching ratio - from the theoretical cross sections which have been obtained using the set-up described in [205]. From these yields, we derived the expected uncertainties shown in the figures. The blue and grey bands (and hatched zones) correspond to two IC models. As can be

\footnotetext{
26 As we mentioned in Section 4.2.2, LHCb using the SMOG system has recently reported a charm production measurement [168] which in principle should, as we advocate here, constrain the IC magnitude. Unfortunately, in the absence of an $H$ target and of a thorough data meta-analysis including theory uncertainty, such current constraints are in fact very weak.
} 


\section{Table 14}

Isolated $J / \psi+\gamma$ production for three bins in the c.m.s. rapidity of the pair. From left to right: the average $x_{2}$, the partial contributions to the cross section from the $g g$-initiated and $q \bar{q}$-initiated subprocesses (multiplied by the $J / \psi$ branching into di-muons) [73] respectively, and the order of magnitude of the expected number of events per year assuming a luminosity of $10 \mathrm{fb}^{-1}$ and a detector efficiency on the order of unity.

\begin{tabular}{lllll}
\hline Isolated $J / \psi+\gamma$ & $\left\langle x_{2}\right\rangle \sim \frac{M_{\psi \gamma}}{\sqrt{s}} e^{-Y_{\psi \gamma}}$ & $\sigma_{g g} \times \mathcal{B}_{\mu \mu}[\mathrm{fb}]$ & $\sigma_{q \bar{q}} \times \mathcal{B}_{\mu \mu}[\mathrm{fb}]$ & $\mathcal{O}(0.2)$ \\
\hline$\left|Y_{\psi \gamma}^{\text {c.m.s. }}\right|<0.5$ & 0.10 & $\mathcal{O}(100)$ & $\mathcal{O}(1000)$ & $\mathcal{O}(0.2)$ \\
$-1.5<\left|Y_{\psi \gamma}^{\text {c.m.s. }}\right|<-0.5$ & 0.25 & $\mathcal{O}(50)$ & $\mathcal{O}(500)$ & $\mathcal{O}(0.04)$ \\
$-2.5<\left|Y_{\psi \gamma}^{\text {c.m.s. }}\right|<-1.5$ & 0.60 & $\mathcal{O}(10)$ & $(10)$ \\
\hline
\end{tabular}

\section{Table 15}

$J / \psi+J / \psi$ production for five bins in the laboratory rapidity of the pair. From left to right: the average $x_{2}$, the partial contributions to the cross section from the gg-initiated and $q \bar{q}$-initiated sub-processes (with the $J / \psi$ branchings into di-muons) and the order of magnitude of the expected number of events (via di-muon pairs) per year assuming a luminosity of $10 \mathrm{fb}^{-1}$ and a detector efficiency on the order of unity.

\begin{tabular}{lllll}
\hline$J / \psi+J / \psi$ & $\left\langle x_{2}\right\rangle \sim \frac{M_{\psi \psi}}{\sqrt{s}} e^{-Y_{\psi \psi}^{\text {c.m.s. }}}$ & $\sigma_{g g} \times \mathcal{B}_{\mu \mu}^{2}[\mathrm{fb}]$ & $\sigma_{q \bar{q}} \times \mathcal{B}_{\mu \mu}^{2}[\mathrm{fb}]$ & Counts/year \\
\hline $4.5<Y_{\psi \psi}^{\text {lab. }}<5.0$ & 0.13 & $\mathcal{O}(5)$ & $\mathcal{O}(1)$ & $\mathcal{O}(50)$ \\
$4.0<Y_{\psi \psi}^{\text {lab. }}<4.5$ & 0.29 & $\mathcal{O}(50)$ & $\mathcal{O}(10)$ & $\mathcal{O}(500)$ \\
$3.5<Y_{\psi \psi}^{\text {lab. }}<4.0$ & 0.45 & $\mathcal{O}(50)$ & $\mathcal{O}(10)$ & $\mathcal{O}(500)$ \\
$3.0<Y_{\psi \psi}^{\text {lab. }}<3.5$ & 0.60 & $\mathcal{O}(10)$ & $\mathcal{O}(10)$ & $\mathcal{O}(100)$ \\
$2.5<Y_{\psi \psi}^{\text {lab. }}<3.0$ & 0.77 & $\mathcal{O}(5)$ & $\mathcal{O}(2)$ & $\mathcal{O}(70)$ \\
\hline
\end{tabular}

seen, even for $P_{T, D^{0}} \lesssim 15 \mathrm{GeV}$ the expected precision of the measurement will clearly allow one to considerably constrain such IC model, by up to an order magnitude. At such large $x$, the perturbative charm is indeed suppressed.

The gluon PDF at high $x$. The available data from DIS structure functions and from DY lepton-pair production provide only rather weak constraints on the gluon PDF, particularly at high $x$. Important information on the gluon density can be drawn from inclusive-jet data and from top-quark-pair-production data. In the latter case, the differential distributions $\left(y_{t \bar{t}}, y_{t}\right.$, $p_{T}^{t}, m_{t \bar{t}}$ ) have been shown to considerably reduce the uncertainty of the gluon PDF at high $x$ [222]. Nevertheless, these data provide constraints at quite large factorisation scales $\mu_{F} \sim 100 \mathrm{GeV}$ and the knowledge of the gluon PDF at $x \gtrsim 0.5$ remains limited.

Let us discuss here a number of possibilities to obtain information on the gluon PDF offered by the AFTER@LHC programme. As can be seen in Fig. 3, open heavy-flavoured mesons and heavy quarkonia will be abundantly produced at AFTER@LHC covering an important region in the $\left(x, m_{T}\right)$ plane. As should be clear from the discussion above on the charm quark PDF, the gluon and the heavy-quark distributions are inextricably linked. Therefore, in the context of a global analysis, the heavy-quark data have the potential to constrain both the heavy-quark and the gluon distribution at high $x$. It should also be noted that, in the case of $b$ quark production, the contribution from an intrinsic bottom component is expected to be very small [201]. One should mention that normalised rapidity distributions of $D$ and $B$ mesons from LHCb have been already used with success to study low- $x$ gluon $[223,224]$.

In addition to the inclusive heavy-quark observables, the associated production of a heavy quarkonium with a photon is sensitive to the high- $x$ gluon distribution. This is illustrated in Table 14 which shows that this process is largely dominated by the gluon-gluon initiated subprocess probing $x_{2}$ values in the range from 0.1 to 0.6 , whereas at such large $x$ single-inclusive-quarkonium production is presumably largely from (heavy) quark induced channels.

Along these lines one can also study double- $J / \psi$ production which is dominated by the gg-channel up to large values of the target $x_{2}$ as is shown in Table 15. It is also interesting to notice that AFTER@LHC provides the unique opportunity to study double parton scatterings and double parton correlations in the nucleon at energies around $100 \mathrm{GeV}$ via di- $/ \psi$ production [225], where the similar studies already exist at the Tevatron and the LHC [226,227].

Another interesting observable which probes the gluon distribution directly at LO via the $q g \rightarrow \gamma q$ subprocess is inclusive prompt-photon production. It was shown that the theoretical pQCD predictions at NLO describe well the collider data for c.m.s. energies ranging from $200 \mathrm{GeV}$ to $7 \mathrm{TeV}$ [228]. However, a series of measurements carried out at c.m.s. energies $\sqrt{s} \sim 20 \div 40 \mathrm{GeV}$ by the fixed-target E706 experiment [229-231] were not well described by NLO pQCD calculations [232-234]. This discrepancy was only partially cured by the inclusion of resummed soft-gluon contributions in the theoretical predictions [235-239]. Therefore, a new measurement at AFTER@LHC at $\sqrt{s}=115$ GeV would be interesting in itself to shed additional light on the data-theory discrepancy at fixed-target energies. Furthermore, such a measurement has the potential to improve our knowledge of the gluon distribution at $x>0.3$ provided isolated photons can be measured with transverse momentum $p_{T} \gtrsim 10 \div 20 \mathrm{GeV}[228]$.

\subsubsection{Nuclear structure}

As a fixed-target experiment, AFTER@LHC allows one to study $p A$ collisions with different nuclei $A$ and some fundamental open questions can be addressed in this case. More than 30 years ago, the EMC collaboration discovered that nuclear structure functions in DIS are suppressed compared to the prediction from the naive combination of free 


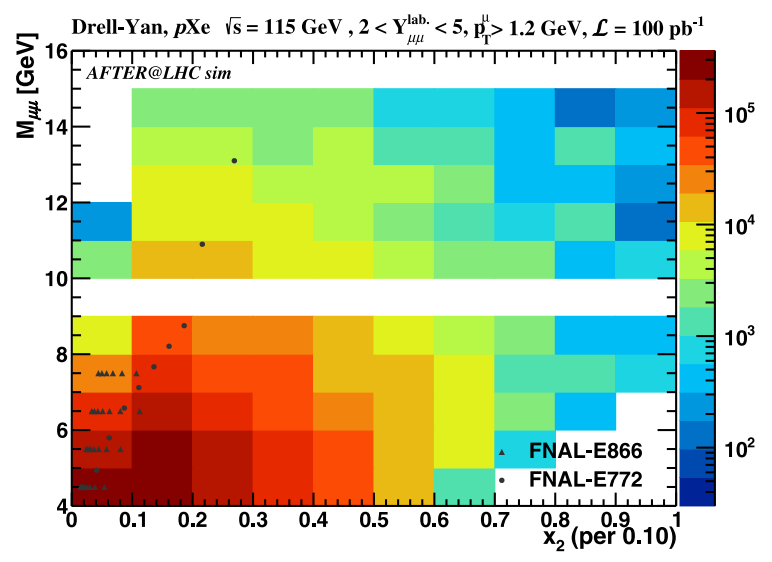

(a)

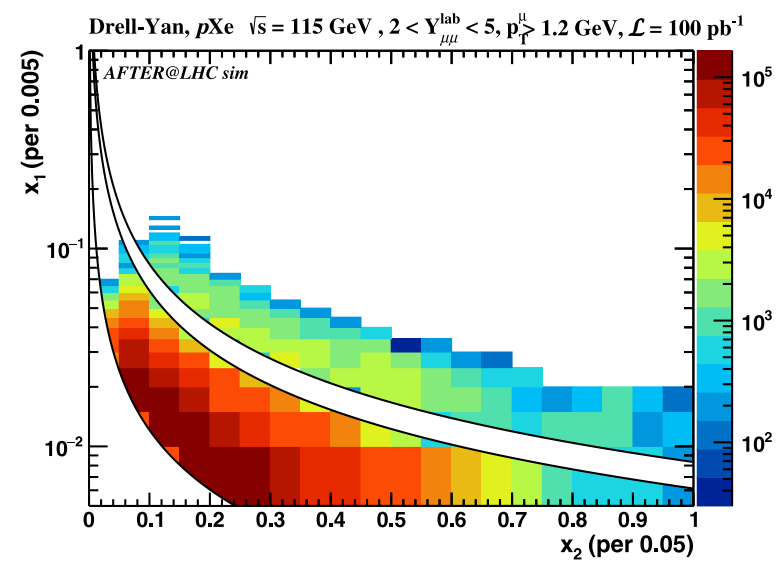

(b)

Fig. 21. Kinematical acceptance for DY lepton-pair production with AFTER@LHCb in $p$ Xe collisions at $\sqrt{s_{N N}}=115 \mathrm{GeV}$ with a muon-pair acceptance of $2<Y_{\mu \mu}^{\text {lab. }}<5$ and single-muon requirements: $2<\eta_{\mu}^{\text {lab. }}<5$ and $p_{T}^{\mu}>1.2 \mathrm{GeV}$. Projections for $p$ Xe collisions are done by applying a nuclear scaling factor $\left(A_{\mathrm{Xe}}\right)$ to the cross sections obtained from the $p p$ simulations. [For $\mathcal{L}_{p \mathrm{Xe}}=\mathcal{L}_{p p}$, the $p$ Xe yields are thus $A_{\mathrm{Xe}}$ times larger than the $p p$ yields]. (a) Di-muon invariant mass vs. $x_{2}$ compared to the existing DY data [180-183] used in current global PDF fits. (b) $x_{1}$ vs $x_{2}$ for the considered di-muon invariant-mass range of $4 \mathrm{GeV}<M_{(\ell \ell)}<15 \mathrm{GeV}$ excluding $\Upsilon$ mass range of $9 \mathrm{GeV}<M_{(\ell \ell)}<10.5 \mathrm{GeV}$. Colours correspond to expected yields of the DY signal in each kinematical region, and each coloured cell contains at least 30 events.

proton and neutron structure functions in the high- $x$ region [4]. The physics mechanism behind this EMC effect is still not fully understood and subject of an active experimental programme at the Jefferson Laboratory [240,241].

A modern precision measurement of the DY lepton-pair production at AFTER@LHC would allow one to study the EMC effect in this process (at high negative $x_{F}$ and with much higher precision) and to compare it to the DIS case. In addition, as in the nucleon case, nuclear PDFs (nPDFs) are determined in global analyses of DIS and DY data [7-10,242,243] and are a crucial ingredient to predict cross sections for hard processes in $p A$ and $A A$ collisions at the LHC. Compared to the proton PDFs, the nPDF determinations are clearly lagging behind both at the level of sophistication but, most importantly, due to the much smaller number of experimental constraints. Currently, the analyses are statistically dominated by DIS data with only about 90 data points from the DY process entering the fits. Incorporating data from various processes is essential for flavour separation in PDF analyses. Therefore, the access to the DY data with a wide kinematic coverage will provide a unique opportunity not only for more precise PDF determinations but will also allow one to test their universality which is a fundamental property of QCD and the basis for all high energy hadron scattering computations.

The kinematic reach of AFTER@LHC (Fig. 21) would allow one to probe much higher $x_{2}$ (target $x$ ) values than the currently available data (data points in Fig. 21) for a variety of targets. In particular, AFTER@LHC could shed new light on the origin of the EMC effect by verifying its presence/absence in DY lepton-pair production. As Fig. 21 shows, a modern precision measurement of DY lepton-pair production at AFTER@LHC covering a wide range in invariant masses of the lepton pairs and extending to higher $x_{F}$ would lead to significant improvements over the current state of the art and would be complementary to results from a future Electron-Ion-Collider (EIC). Clearly, it would be an invaluable input for nuclear PDF determinations.

As an example we present here a reweighting analysis [245-248] showing the potential impact of the DY lepton-pair production data from AFTER@LHC in pXe collisions on the nCTEQ15 nPDFs. In this analysis, we have used pseudo-data for the nuclear modification factors $R_{p \mathrm{XY}}^{\mathrm{DY}}=\sigma_{\mathrm{DY}}^{\mathrm{Xe}} /\left(A \times \sigma_{\mathrm{DY}}^{p p}\right)$ as a function of the rapidity in five bins of lepton-pair invariant mass: $4 \mathrm{GeV}<M_{\mu \mu}<5 \mathrm{GeV}, 5 \mathrm{GeV}<M_{\mu \mu}<6 \mathrm{GeV}, 6 \mathrm{GeV}<M_{\mu \mu}<7 \mathrm{GeV}, 7 \mathrm{GeV}<M_{\mu \mu}<8 \mathrm{GeV}$ and $M_{\mu \mu}>10.5 \mathrm{GeV}$, in order to estimate the effect these data can have on the current nPDFs. A selection of the pseudo-data together with the corresponding theory predictions is shown in Fig. 22, where we have assumed the following luminosities: $\mathcal{L}_{\mathrm{pp}}=10 \mathrm{fb}^{-1}$ and $\mathcal{L}_{\mathrm{pXe}}=100 \mathrm{pb}^{-1}$ for the pseudo-data.

In Fig. 23, we display the nPDFs before and after the reweighting using the AFTER@LHC $R_{p X e}$ pseudo-data. We can see a significant decrease of the errors for up and down quark distributions showing the potential of the AFTER@LHC to constrain nPDFs. In practice, due to the limited amount of data, the current nPDF errors are considerably underestimated and the actual importance of these data cannot be fully demonstrated in this kind of study. However, Fig. 21 clearly shows how complementary the kinematical coverage of AFTER@LHC will be compared to the current DY data for the $\mathrm{nPDF}$ determination. Similarly to the proton case, the $W^{ \pm}$data could be used for a determination of the high- $x$ nPDF in particular the light-quark-sea distributions.

Additionally, we have also investigated what the impact of such DY measurements on nPDFs would be in case less data would be collected. For this purpose, we have assumed 10 times reduced luminosities for both $p p$ and $p$ Xe samples. The results for such a scenario are presented in Fig. 24 which shows that even in this case one can obtain a substantial reduction of uncertainties for up and down distributions. 


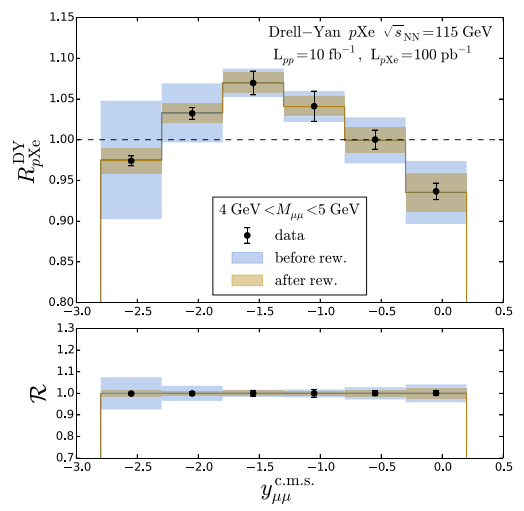

(a) $4 \mathrm{GeV}<M_{\mu \mu}<5 \mathrm{GeV}$

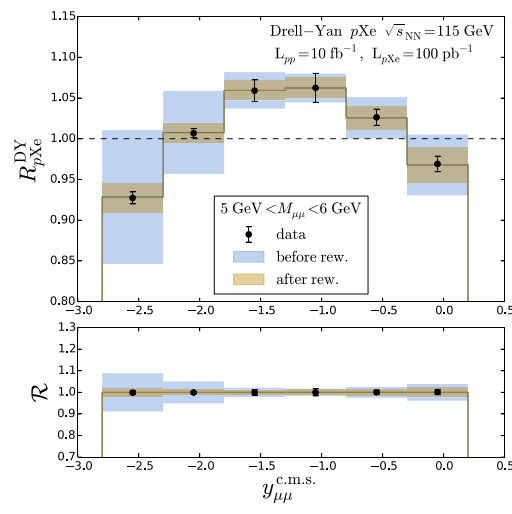

(b) $5 \mathrm{GeV}<M_{\mu \mu}<6 \mathrm{GeV}$

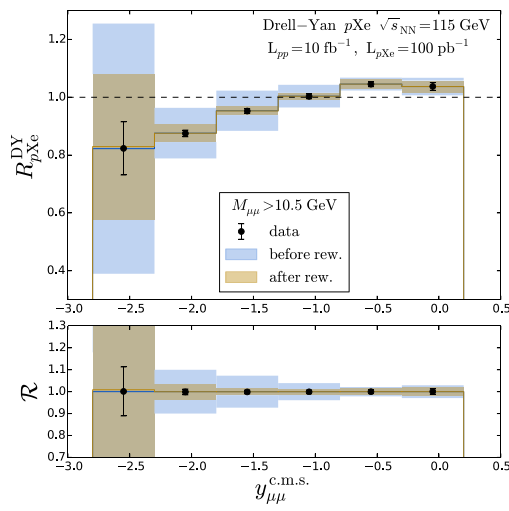

(c) $M_{\mu \mu}>10.5 \mathrm{GeV}$

Fig. 22. Projection of the statistical uncertainties on the nuclear modification factor $R_{p \mathrm{~A}}=\sigma_{\mathrm{DY}}^{\mathrm{Xe}} /\left(A \times \sigma_{\mathrm{DY}}^{p p}\right)$ for $\mathrm{DY}$ lepton-pair production in $p$ Xe collisions in different mass ranges compared to the uncertainties encoded in nCTEQ15 nPDFs (in blue 'before rew.'), which are representative of typical nPDF uncertainties. The projected statistical uncertainties arise from the subtraction of the uncorrelated, combinational background (based on the like-sign technique) and assuming the yearly integrated luminosities of $\mathcal{L}_{p p}=10 \mathrm{fb}^{-1}$ and $\mathcal{L}_{p \mathrm{Xe}}=100 \mathrm{pb}^{-1}$. The brown band ('after rew.') corresponds to the uncertainty of the $R_{p A}$ after a Bayesian reweighting of the nPDF using the corresponding pseudo-data.

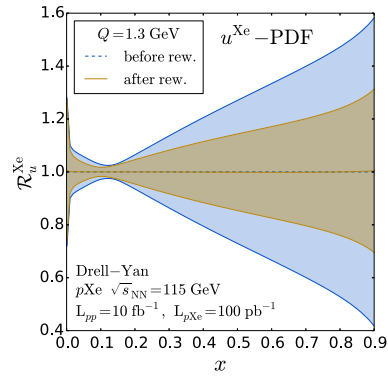

(a)

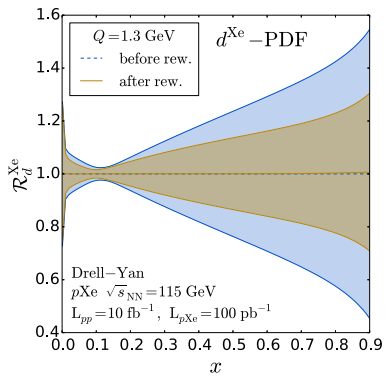

(b)

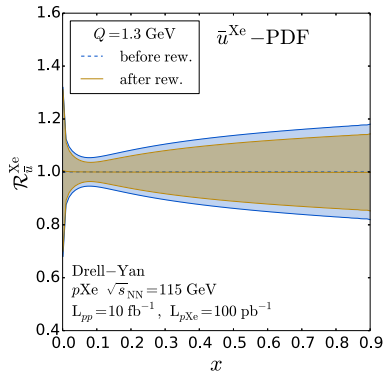

(c)

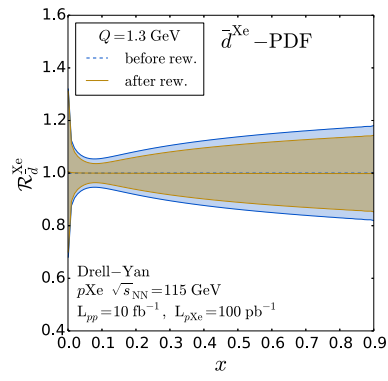

(d)

Fig. 23. Uncertainty on nCTEQ15 nPDFs before and after the reweighting using $R_{p X e}^{\mathrm{DY}}$ AFTER@LHCb pseudo-data in the range indicated in Fig. 21. The plots show ratio of nPDFs for tungsten $(W)$ and the corresponding uncertainties compared to the central value at the scale $Q=1.3$ GeV.

AFTER@LHC is also able to constrain the high- $x$ nuclear gluon distribution, which is the least known nPDF. A prime example we show here is to use heavy-flavour production at AFTER@LHC, where the gluon shadowing effect on $J / \psi$ and $\Upsilon$ production in $p \mathrm{~Pb}$ collisions at AFTER@LHC energies has been studied in Ref. [249]. We now discuss the potential of both open and hidden heavy-flavour mesons $\left(D^{0}, J / \psi, B^{+}, \Upsilon(1 S)\right)$ production in $p$ Xe collision at $\sqrt{s_{N N}}=115 \mathrm{GeV}$ to pin down the high- $x$ gluon density in nPDF by performing a Bayesian-reweighting analysis. A similar study in the LHC energies has been carried out in Ref. [244]. We have used the data-driven approach proposed in Ref. [250] to fit matrix elements of the heavy flavour hadrons, and then folded them with the proton CT14 PDFs and nCTEQ15 [8] nPDFs to get the yields and the nuclear modification factors. 27

The pseudo-data are generated to match the central theoretical predictions. Their projected statistical uncertainties are estimated by assuming the yearly integrated luminosities $\mathcal{L}_{p p}=10 \mathrm{fb}^{-1}, \mathcal{L}_{p \mathrm{Xe}}=100 \mathrm{pb}^{-1}$ and the reconstruction efficiency $\varepsilon=0.1$. The branching ratios of $D^{0} \rightarrow K \pi, B^{+} \rightarrow K\left(J / \psi \rightarrow \mu^{+} \mu^{-}\right), J / \psi \rightarrow \mu^{+} \mu^{-}$and $\Upsilon(1 S) \rightarrow \mu^{+} \mu^{-}$have also been taken into account. For $D^{0}$ and $B^{+}$, their charge-conjugated particles are also summed up. After considering a $2 \%$ systematic error and a $5 \%$ global error, the nuclear modification factors $R_{p X e}$ for the four hadron productions are shown in Fig. 25 before (red bands) and after (blue bands) reweighting, together with the variations of factorisation scales. The uncertainty from the factorisation scale can be the dominant theoretical error after nPDF reweighting as already pointed out in Ref. [244]. The impact of these pseudo-data can be transferred into nPDFs as illustrated in Fig. 26 and 27. We can see that even accounting for the scale uncertainty, a substantial reduction of nPDF uncertainty at high- $x$ values $(x \gtrsim 0.3)$ is still achieved. It clearly demonstrates the uniqueness of the AFTER@LHC programme in exploring gluon densities in nuclei especially at high $x$. 


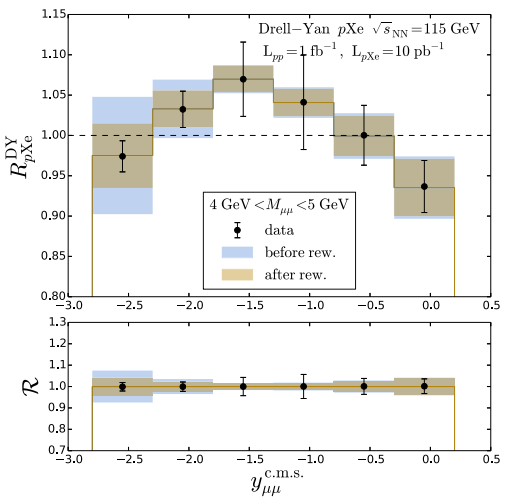

(a) $4 \mathrm{GeV}<M_{\mu \mu}<5 \mathrm{GeV}$

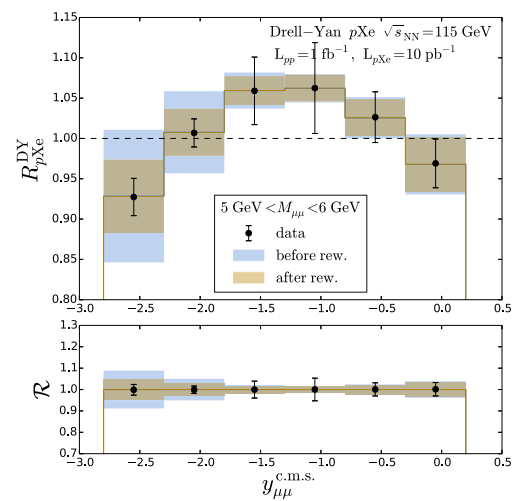

(b) $5 \mathrm{GeV}<M_{\mu \mu}<6 \mathrm{GeV}$

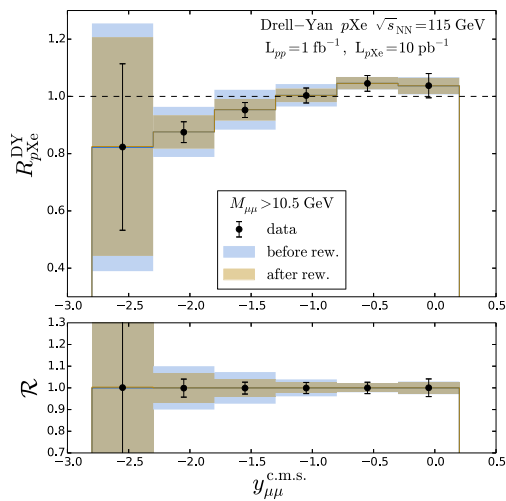

(c) $M_{\mu \mu}>10.5 \mathrm{GeV}$

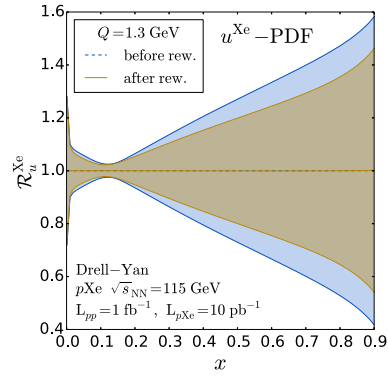

(d)

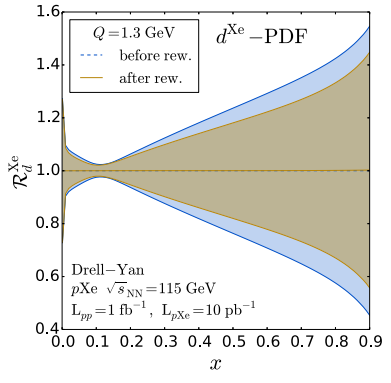

(e)

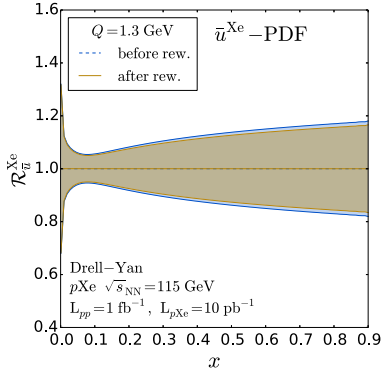

(f)

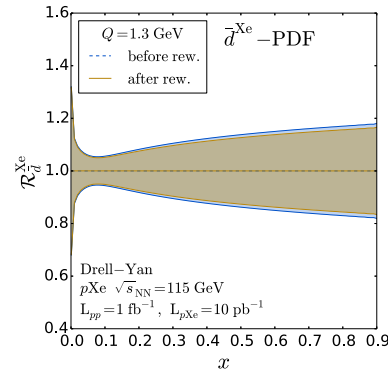

$(\mathrm{g})$

Fig. 24. Same as Fig. 22 (upper row) and Fig. 23 (lower row) with luminosities reduced by a factor $10: \mathcal{L}_{p p}=1 \mathrm{fb}^{-1}$ and $\mathcal{L}_{p \times e}=10 \mathrm{pb}^{-1}$.

It is important to note here that the above projections for the constraints on the gluon nPDF were obtained assuming only the modification of nPDFs and the absence of other cold nuclear matter effects, or that such other effects can be subtracted. At LHC collider energies, this kind of leading-twist-factorisation approach was applied with success to a large class of existing data [244]. At lower energies, especially in the backward region, quarkonium break up will likely play a role and should be separated out. For that matter, the extensive access of AFTER@LHC to quarkonium excited-state studies will be crucial. Another example of an effect that can matter when gluons are involved is the coherent energy loss. It was recently studied in the context of AFTER@LHC [253] and results in a modification of the pA cross-sections compared to the $p p$ one which is depicted in Fig. 28 for $J / \psi$ and $\Upsilon$ in terms of $R_{p A}$. Combining all the heavy-flavour related measurements possible at AFTER@LHC will certainly allow one to disentangle all these effects in order to perform a reliable fit of the gluon nPDFs using these data.

\subsubsection{Astroparticle physics}

Recently, measurements of cosmic rays (CRs) with very high energies, ranging from about tens of MeV up to hundreds of TeV, became possible for many particle species ( $e^{ \pm}[254,255], \gamma$ [256,257], $v$ [258,259], $p$ [260], $\bar{p}$ [261], $A$ [262-264]) and attracted much attention. The mechanism responsible for the generation of such Ultra High-Energy CRs (UHECRs) is still under intense discussion, with two main scenarios: (i) the acceleration of particles due to astrophysical phenomena and (ii) dark matter decay/annihilation. The mechanism generating CRs can only be determined if we can identify characteristic shapes of the spectrum such as sharp cutoffs which will indicate the decay of massive dark matter particles. In such precision tests of CRs, the spectrum has to be accurately determined, thus naturally requiring precise investigations of other sources acting as backgrounds. Here we present two cases where the AFTER@LHC programme can play a critical role.

UHECR neutrinos and the proton charm content. The terrestrial observation of UHE neutrinos lately became possible thanks to IceCube, with the highest energy recorded on the order of PeV [258,259]. Atmospheric neutrinos, generated by the weak decays of final-state particles of the collisions between CRs and atmospheric nuclei, are however an important background to these ground observations of cosmic neutrinos. The major source of these atmospheric neutrinos is from the weak decay of hadrons. Those originating from the decay of long-lived mesons, like $\pi$ and $K$ mesons, dominate the energy spectrum below $10^{5} \mathrm{GeV}$. Those with energy above $10^{5} \mathrm{GeV}$ are from charm-hadron decays. Indeed charmed hadrons lose significantly less energies in the atmosphere than $\pi$ and $K$ because they decay almost instantaneously. 


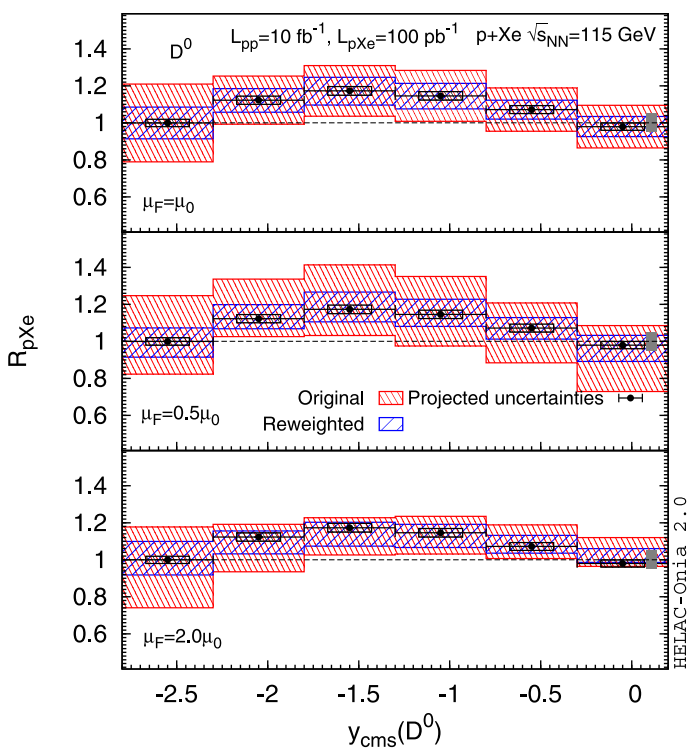

(a)

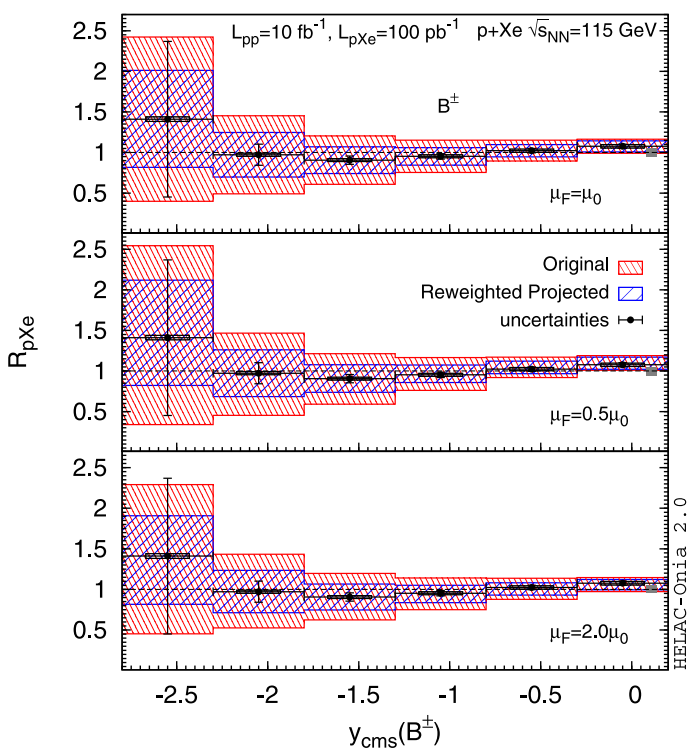

(c)

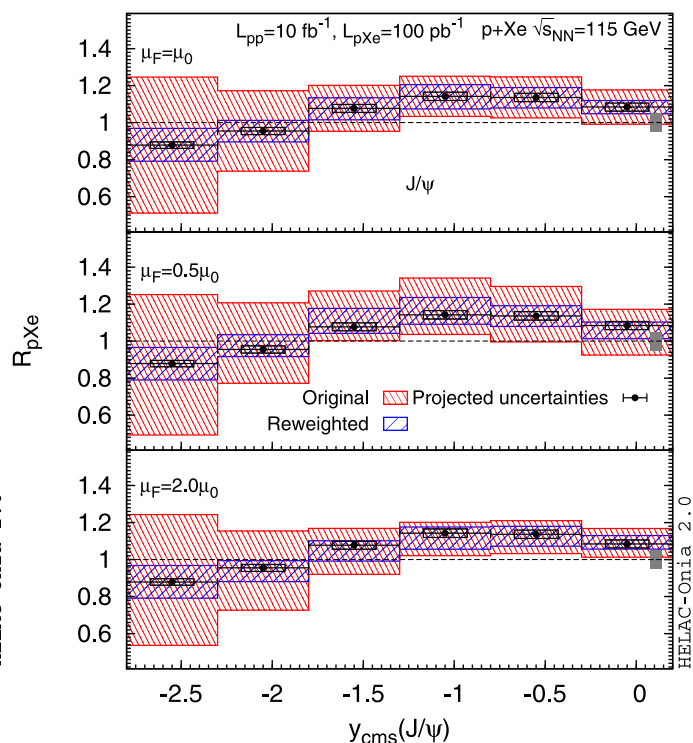

(b)

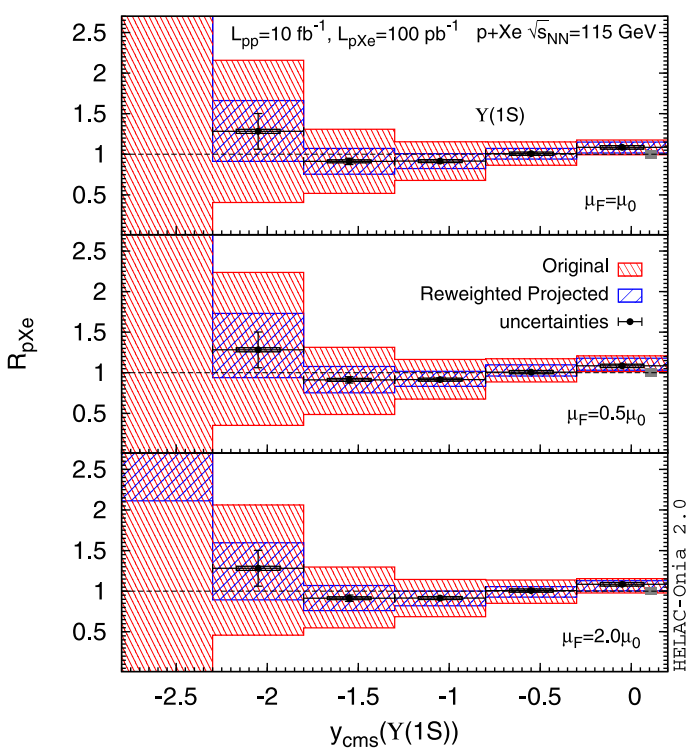

(d)

Fig. 25. Projection of the statistical uncertainties on the $R_{p A}$ for (a) $D^{0}$, (b) $J / \psi$, (c) $B^{+}$, and (d) $\Upsilon$ (1S) production in pXe collisions compared to the uncertainties encoded in nCTEQ15 nPDFs, which are representative of typical nPDF uncertainties, evaluated at different typical choices of the factorisation scale, $\mu_{F}$, varied about $\mu_{0}$ like in [244]. A $2 \%$ uncorrelated systematic uncertainty and a $5 \%$ global uncertainty are also shown. The projected statistical uncertainties are estimated assuming the yearly integrated luminosities of $\mathcal{L}_{\mathrm{pp}}=10 \mathrm{fb}^{-1}$ and $\mathcal{L}_{\mathrm{pXe}}=100 \mathrm{pb}^{-1}$.

The yield of neutrinos from charm naturally follows that of charmed hadrons produced in the collisions of UHECRs and atmospheric nuclei. An accurate evaluation of the charm hadroproduction cross section is therefore crucial to assess their importance. The charm production from $p p$ scattering was first evaluated in perturbative approaches, which considered charm quark-anti-quark pairs virtually created by the gluon splitting $g \rightarrow c \bar{c}$. This contribution is mostly relevant at low $x$ in the target PDF $[265,266]$ which can also be shadowed.

The relevance of a nonperturbative charm content (or IC) in the projectile proton was also recently considered [267]. Just as most global analyses of PDFs rely on the assumption that the charm and bottom PDFs are generated perturbatively by gluon splitting, most of the studies of neutrino fluxes were based on the same assumption. The intrinsic charm however has a harder distribution [202,203] (for a recent review, see [205]) and tends to predict charmed hadrons at higher energies, which would result into higher energy neutrinos. 


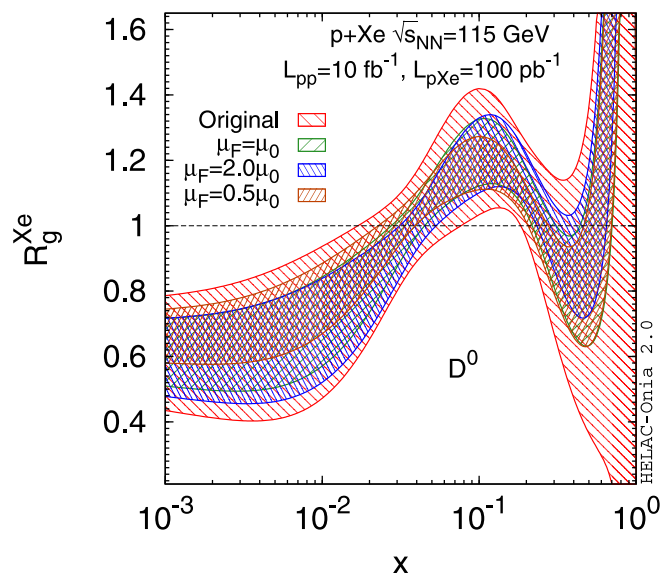

(a)

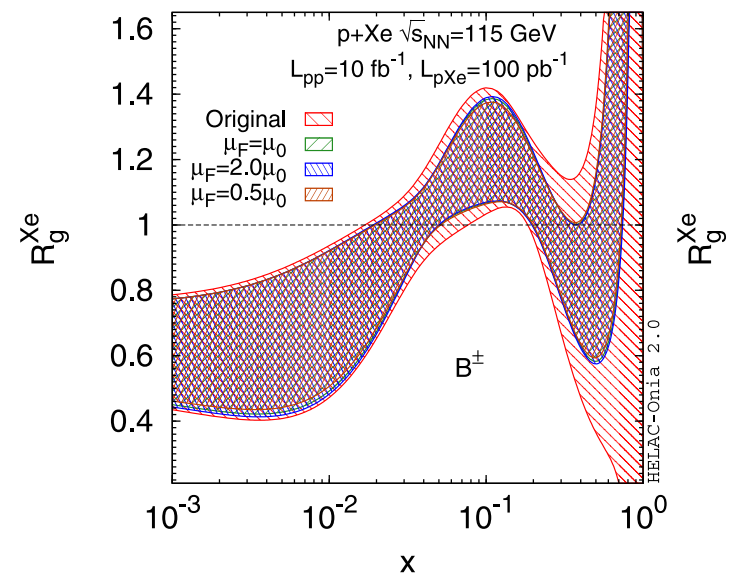

(c)

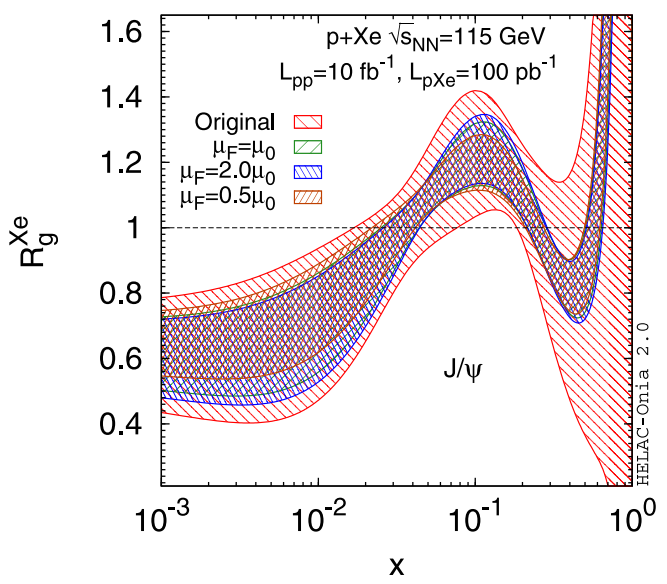

(b)

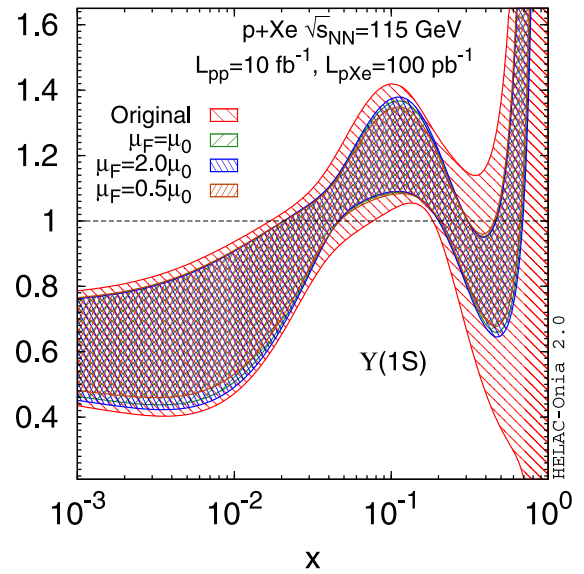

(d)

Fig. 26. nCTEQ15 nPDFs before and after the reweighting using $R_{p X e}$ pseudo-data shown in Fig. 25 for (a) $D^{0}$, (b) $J / \psi$, (c) $B^{+}$, (d) $\Upsilon(1 S)$ production at AFTER@LHCb. The plots show ratios $R_{g}^{X e}$ of gluon densities encoded in nCTEQ15 over that in CT14 PDFs at scale $Q=2 \mathrm{GeV}$.

As we discussed in Section 5.1.1, the AFTER@LHC programme offers a novel playground to study the excess of charm at high $x$. Not only a detector such as that of LHCb used in the fixed-target mode can access the high negative $x_{F}$, it also has a wide coverage close to $40 \%$ [268] of the weighted charm cross section, $x_{F}^{2} d \sigma / d x_{F}$ which is quasi proportional to the neutrino flux. As a comparison, in the collider mode, it covers less than $10 \%$ of the same weighted cross section [269]. In other words, the charmed hadron measurements of the AFTER@LHC programme can provide the most decisive constraints on the non-perturbative charm content in the proton in the region relevant to understand the neutrino-from-charm flux. Fig. 29 shows the uncertainties on the neutrino flux due to that on the IC in the projectile proton [270] (see also [267]).

Anti-proton cross section and UHECR Monte-Carlo tuning. Among the cosmic rays, the anti-protons $(\bar{p})$ are the object of a specific attention. In the current understanding, $\bar{p}$ are almost always of secondary origin, i.e. created through high-energy scatterings between the interstellar matter and primary cosmic rays, which were mainly generated by acceleration in supernova remnants [271-273]. Cosmic $\bar{p}$ were measured by the AMS-02 experiment in the range of $1 \mathrm{GeV}$ to $400 \mathrm{GeV}$ with a typical accuracy of 5\% [261]. On the theory side, the spectrum of secondary $\bar{p}$ can be predicted using diffusion equations with the $\bar{p}$ production cross section as input [274-276]. The determination of the discrepancy between the above two can open a new window on the indirect detection of dark matter or unknown astrophysical mechanisms of CR acceleration.

The accurate evaluation of the cosmic $\bar{p}$ spectrum requires a precise knowledge of their production cross section for several nuclear channels. The nuclear primary CRs are composed of $p,{ }^{4} \mathrm{He},{ }^{12} \mathrm{C},{ }^{14} \mathrm{~N},{ }^{16} \mathrm{O}$. Other nuclei need not to be considered due to their small contribution to $\bar{p}$ production. Moreover, the interstellar matter, which acts as a fixed target for the cosmic $\bar{p}$ production, is composed of $p$ and ${ }^{4} \mathrm{He}$. Other nuclei acting as targets are negligible. The estimation of the contribution of $\bar{p}$-production cross sections for each nuclear channel to the $\bar{p}$ spectrum is roughly $50 \%$ for $p+p \rightarrow \bar{p}+X$, 


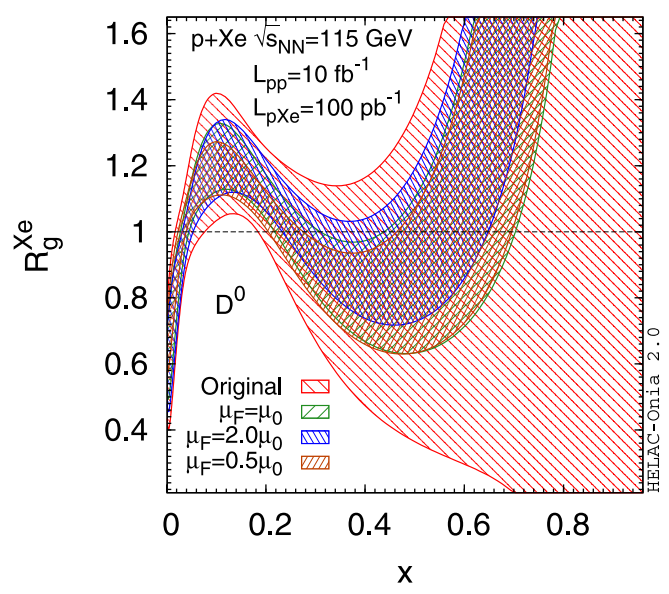

(a)

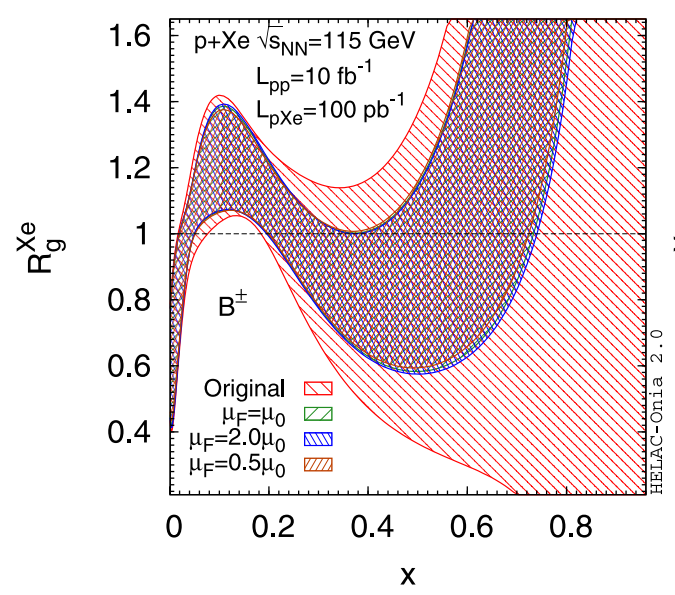

(c)

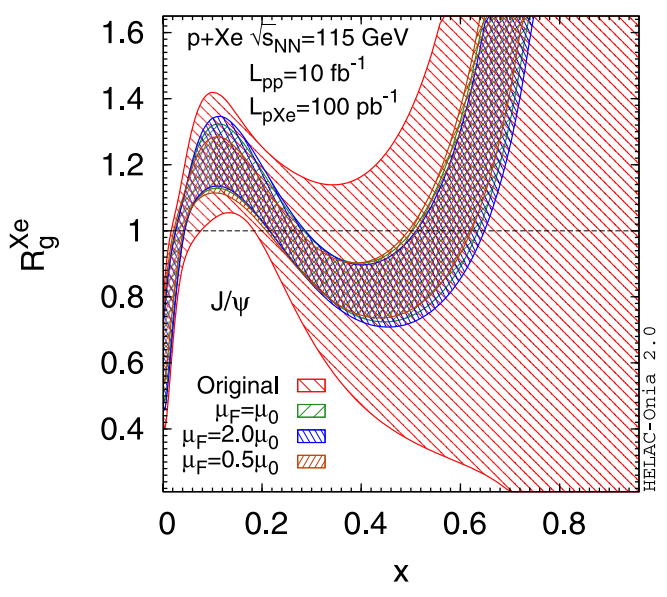

(b)

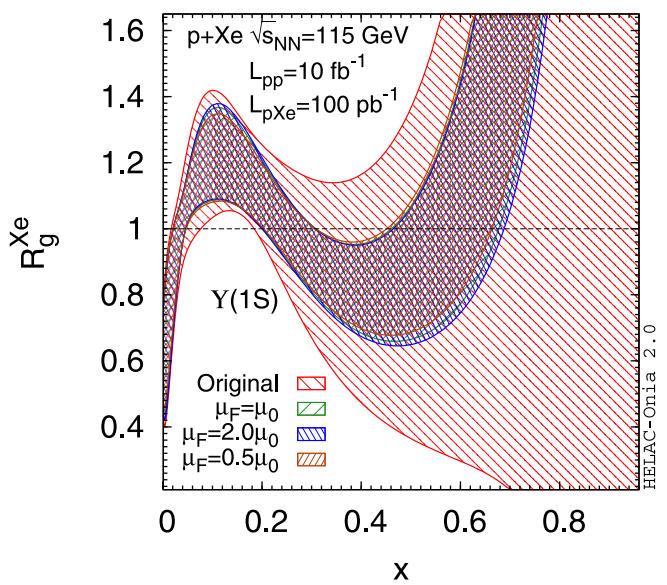

(d)

Fig. 27. Same as Fig. 26 using a linear $x$ axis in order to highlight the high- $x$ region.
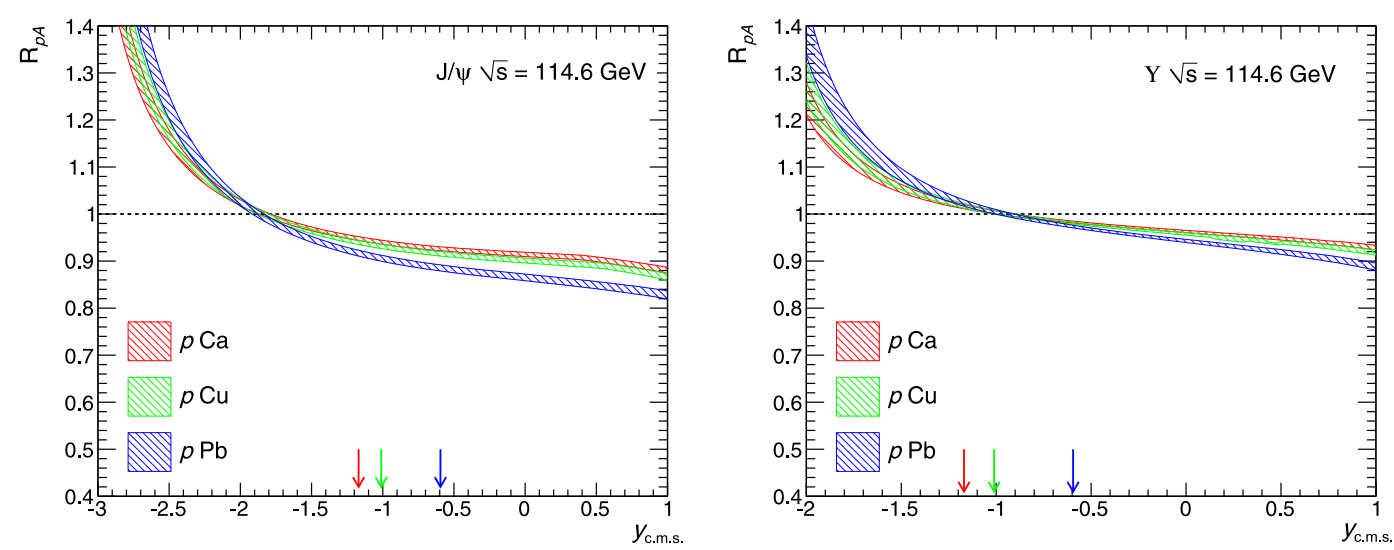

Fig. 28. $J / \psi$ (left) and $\Upsilon$ (right) nuclear modification factor in $p \mathrm{~Pb}, p \mathrm{Ca}$, and $p \mathrm{Cu}$ collisions at $\sqrt{s}=114.6 \mathrm{GeV}$ resulting from the coherent energy loss.

Source: Adapted from [253].

$30 \%-40 \%$ for $p+{ }^{4} \mathrm{He} \rightarrow \bar{p}+X$ ( $p,{ }^{4} \mathrm{He}$ can either be the target or the projectile), $5 \%$ for ${ }^{4} \mathrm{He}+{ }^{4} \mathrm{He} \rightarrow \bar{p}+X$, and $5 \%$ for $p+A \rightarrow \bar{p}+X$ with $A={ }^{12} \mathrm{C},{ }^{14} \mathrm{~N},{ }^{16} \mathrm{O}[277]$. The other channels contribute in all less than $5 \%$. 


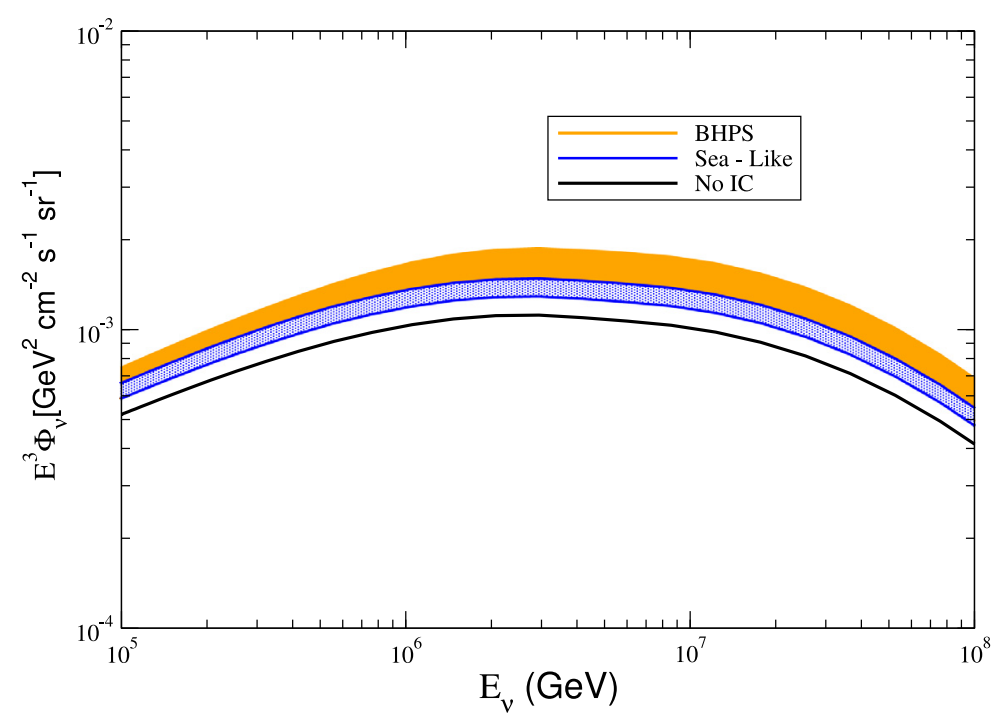

Fig. 29. Impact of the uncertainties on the charm content of the proton on the neutrino flux [Courtesy of V. Gonçalves, based on [270]]. The uncertainties on the $D$ yield correspond to Fig. 20 with the use of the BHPS and sea-like IC.

Here we note that, in unveiling new astrophysical phenomena, the most energetic region of the cosmic $\bar{p}$ spectrum is the most interesting, since the determination of the high-energy CR source (whether it is due to dark matter decays/annihilations or astrophysical accelerations) relies on the shape of the CR spectrum in the high-energy region. It is thus required to quantify the high-energy part of the production cross section of secondary $\bar{p}$. At the partonic level, the generation of highly energetic $\bar{p}$ is dominantly due to the collision of two gluons with highly asymmetric momentum fractions, and the $\bar{p}$ production cross section off two nuclei is sensitive to the gluon content of the nuclei at high momentum fraction, which is not very well known. Precise measurements of $\bar{p}$ production off nuclei are therefore important to improve quantitative predictions of the cosmic $\bar{p}$ spectrum.

Currently, the production cross sections of $p p \rightarrow \bar{p}+X$ and $p+{ }^{4} \mathrm{He} \rightarrow \bar{p}+X\left({ }^{4} \mathrm{He}\right.$ as target) were measured [116] and [278] in the energy range of AMS-02 [279-281]. In particular, the LHCb experiment with SMOG measured the production of $\bar{p}$ from the scattering of $p$ beam off ${ }^{4} \mathrm{He}$ target in the range of $\bar{p}$ momentum $12 \mathrm{GeV}-110 \mathrm{GeV}[116,277]$ and contributed to the improvement of the prediction of the secondary cosmic $\bar{p}$ spectrum. In this context, we note that AFTER@ALICE $\mathrm{CB}_{\mathrm{C}}$ (i.e. the ALICE Central Barrel (CB) used in the fixed target mode) can measure very slow $\bar{p}$, with almost zero momentum. The production of such slow $\bar{p}$ (which are apparently outside the range measurable by AMS-02) with the LHC $p$ beam corresponds to the highest possible energies in the inverse kinematics, where the nuclear target $(\mathrm{C}, \mathrm{N}, \mathrm{O}, \mathrm{He})$ travels at $\mathrm{TeV}$ energies, hit an interstellar $p$ at rest and produces a $\bar{p}$ in the limit of $x_{F} \rightarrow 1$.

Having this observation in mind and considering any arbitrary stable nucleus as target, let us discuss more precisely the cases which can be studied with AFTER@ALICE ${ }_{C B}$ and AFTER@LHCb assuming the additional possibility of ${ }^{16} \mathrm{O}$ beam, as envisioned at LHC for a short period in Run 3 [282]. Using a gas target, the $p+{ }^{4} \mathrm{He} \rightarrow \bar{p}+X$ process (with $p$ as a projectile) with slow emerging $\bar{p}$ falls within the acceptance of ALICE. Fig. 30 shows the $\bar{p}$ kinematical reach ${ }^{28}$ for the ALICE CB for $p p$ collisions for which the ALICE CB performances are similar as for $p$ He collisions. As expected, $\bar{p}$ with momenta as low as a few hundred $\mathrm{MeV}$ (which correspond to a rapidity difference between the He target and the $\bar{p}, \Delta y_{\mathrm{He}}$, as low as 0.4 ) can easily be detected. Such $\bar{p}$ with small $\Delta y_{\text {He }}$ for ${ }^{4} \mathrm{He}+p \rightarrow \bar{p}+X$ correspond to the high-energy tail of the ${ }^{4} \mathrm{He}$ $+p \rightarrow \bar{p}+X$ process ( ${ }^{4} \mathrm{He}$ as projectile), which is one of the leading process in the cosmic $\bar{p}$ spectrum. Similarly, using $\mathrm{C}, \mathrm{N}$ or $\mathrm{O}$ targets, one can study the high-energy $\bar{p}$ tail for $(\mathrm{C}, \mathrm{N}, \mathrm{O})+p \rightarrow \bar{p}+X$. To the best of our knowledge, no other experimental set-up can cover this high-energy limit.

With an oxygen beam, the study of the low-energy $\bar{p}$ in the laboratory frame from ${ }^{16} \mathrm{O}+p \rightarrow \bar{p}+X$ as well as from ${ }^{16} \mathrm{O}+{ }^{4} \mathrm{He} \rightarrow \bar{p}+X$ processes would become possible; this corresponds to the actual astrophysical situation. In such a case, AFTER@ALICE $E_{C B}$ would be able to access the very low energy domain, whereas the LHCb coverage would be similar as for previous He studies, i.e. from 10 to $100 \mathrm{GeV} .{ }^{16} \mathrm{O}$ is the most abundant nucleus in our universe after $\mathrm{H}$ and $\mathrm{He}$, such that such experimental measurements will likely reduce the uncertainty of the cosmic $\bar{p}$ spectrum.

\footnotetext{
28 The purpose of this plot is to show the kinematical range and the statistics available in the fixed-target set-up. We do not expect to obtain different results by using another Monte-Carlo code such as EPOS for instance.
} 


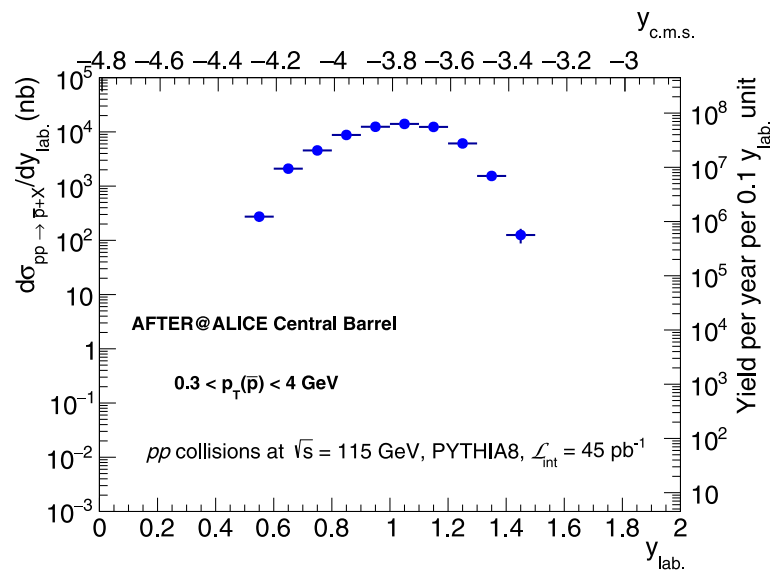

(a) $y_{\text {Lab. }}$ coverage

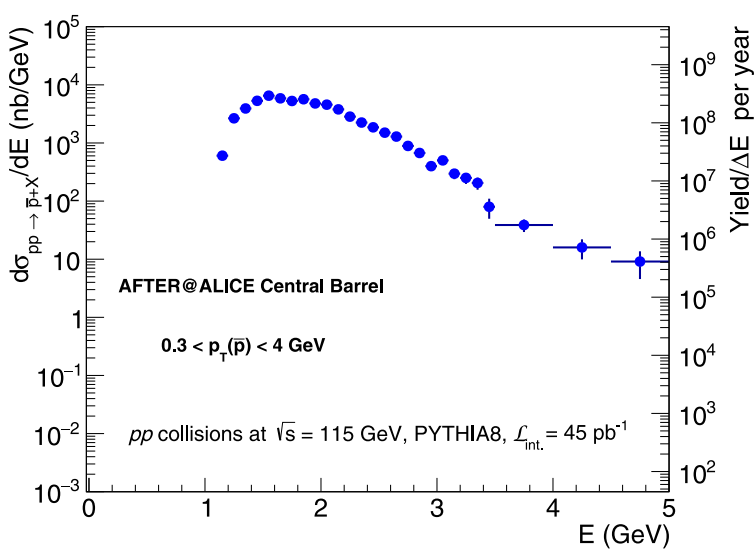

(b) $E$ coverage

Fig. 30. The expected kinematical coverage for $\bar{p}$ production obtained with PYTHIA8. for AFTER@ALICE $\mathrm{CB}_{\text {B }}$ with a displaced vertex at $z_{\text {target }}=-4.7 \mathrm{~m}$ from the nominal ALICE IP. Results are obtained assuming reduced track length in TPC, which gives the TPC acceptance of $1.35<\eta<2.50$ and the TOF acceptance of $0.27<\eta<1.58$.

\subsection{Spin physics}

This section focuses on the case for spin physics at AFTER@LHC, which is very competitive in the worldwide context. It can be divided into two parts. The first relies on the use of a polarised target to carry out many SSA measurements of common probes with a high precision, but also to measure - for the first time - SSA on rare perturbative probes which would remain unaccessible otherwise. ${ }^{29}$ As such, AFTER@LHC opens a novel domain of investigation of the Sivers-like effects for several years, in order to eventually access and (indirectly) constrain the OAM of the gluons and quarks in the proton, but also in the neutron and the deuteron. Such a quest of measuring the parton OAM goes along with a complete tridimensional tomography of the momenta of the partons confined in hadrons.

The second part of the spin physics case relies on the very large luminosities to be accumulated thanks to the fixedtarget mode, combined with an acceptance towards low transverse momenta at high $x$ in the target. This allows one to probe in a systematic way a class of azimuthal asymmetries related to the violation of the Lam-Tung relation [68] in DY production, and potentially [69] to the Boer-Mulders effect [67]. AFTER@LHC also allows one to study its counterpart in the gluon sector, with probably the first systematic measurement of the distribution of the linearly-polarised gluons in unpolarised nucleons at large momentum fractions.

STSAs can be described by nonperturbative twist-2 TMD matrix elements and nonperturbative twist- 3 collinear matrix elements (CT3) in different kinematic regions: the TMD picture holds when there are two separate scales (such as the transverse momentum $q_{T}$ and the invariant mass $M$ of the lepton pair in DY production with $\Lambda_{\mathrm{QCD}} \leq q_{T}<M$ ), whereas the CT3 picture holds when one single hard scale is present (such as the transverse momentum $p_{T}$ of a pion produced in hadronic collisions with $\Lambda_{\mathrm{QCD}}<p_{T}$ ). Both non-perturbative descriptions can in general be related in the overlapping kinematic region in $q_{T}$ by means of coefficients which are perturbatively calculable (see e.g. [283,284] for some caveats in this respect). Both twist-2 TMD and twist-3 collinear matrix element contain essential information on the spin structure of the nucleon and their knowledge is intertwined. AFTER@LHC will test with high precision whether these two formalisms offer the right description of SSAs.

We first discuss the (target) STSAs (i.e. $A_{N} \equiv A_{U T}$ ), both for quark-induced and gluon-induced processes, which give access to both quark and gluon Sivers functions and several 3-parton correlation functions. Then we present the prospects for the measurement of spin-related azimuthal asymmetries in unpolarised hadron collisions, which also probe either the quark or the gluon content of the nucleon. Besides the discussion of spin and azimuthal asymmetries, we also elaborate on the relation between the TMDs that can be constrained by AFTER@LHC and the OAM. Next we discuss the use of ultraperipheral collisions to access GPDs through exclusive photoproduction processes and quarkonium production. Finally we discuss the possibility to constrain the strange-quark-helicity distribution at large $x$ through $\Lambda$ production.

\footnotetext{
29 Let us recall that in this c.m.s. energy range, RHIC offers significantly lower luminosities with a limited access to high $x$ in the polarised nucleons.
} 


\subsubsection{Quark Sivers effect}

The STSA, $A_{N}$, can be experimentally defined (in a schematic way) as ${ }^{30}$

$$
A_{N}=\frac{1}{\mathcal{P}_{\text {eff }}} \frac{\sigma^{\uparrow}-\sigma^{\downarrow}}{\sigma^{\uparrow}+\sigma^{\downarrow}},
$$

where $\sigma^{\uparrow(\downarrow)}$ is a differential-production cross section of particles produced with the target spin polarised upwards (downwards), and $\mathcal{P}_{\text {eff }}$ is the effective polarisation. $A_{N}$ is of particular interest because it was predicted to be small $\left(A_{N} \propto m_{q} / p_{T} \sim O\left(10^{-4}\right)\right.$ in the collinear pQCD approach at the leading twist, while the measured $A_{N}$ reaches 0.1 at high $x_{F}\left(=x_{1}-x_{2}\right)$ in polarised collisions over a broad range of energy [64,286,287]).

As introduced in Section 2, $A_{N}$ can be addressed either using the TMD formalism through the Sivers function, or using the CT3 formalism through 3-parton correlation functions. One of the most important predictions, shared by both approaches, is the sign change of this asymmetry between SIDIS and DY processes. We explain below how AFTER@LHC can contribute to precisely measure this sign change.

Moreover, the accurate measurements to be performed by AFTER@LHC will help constrain the non-perturbative input that enters the TMD evolution kernel [48,288-293], which has an important effect on the STSA (see e.g. [294,295]).

Drell-Yan production. DY lepton-pair production is a unique tool to study the Sivers effect, because it is theoretically very well understood and the Sivers function $f_{1 T}^{\perp q}\left(x, k_{T}^{2}\right)$ for quarks (which represents the difference of number densities of unpolarised quarks with transverse momentum $k_{T}$ and collinear momentum fraction $x$ for a given two opposite configurations of the transverse spin of the proton) is predicted to have an opposite sign for DY and SIDIS processes:

$$
f_{1 T}^{\perp q}\left(x, k_{T}^{2}\right)_{\mathrm{DY}}=-f_{1 T}^{\perp q}\left(x, k_{T}^{2}\right)_{\text {SIDIS }} .
$$

Within the TMD formalism, and up to angular integrations, $A_{N}$ in $p p^{\uparrow}$ collisions can be schematically written as

$$
A_{N} \sim \frac{f_{1}^{q}\left(x_{1}, k_{T 1}^{2}\right) \otimes f_{1 T}^{\perp \bar{q}}\left(x_{2}, k_{T 2}^{2}\right)}{f_{1}^{q}\left(x_{1}, k_{T 1}^{2}\right) \otimes f_{1}^{\bar{q}}\left(x_{2}, k_{T 2}^{2}\right)},
$$

where $f_{1}^{q}$ stands for the unpolarised quark TMD PDF, and $\otimes$ represents a convolution in momentum space and a sum over quark and anti-quark flavours.

The verification of the sign change of the Sivers function is the main physics case of the DY COMPASS programme [78], which recently performed the first measurement of the asymmetry in DY production [81], and the experiments E1039 [77] and E1027 [296] at Fermilab. The AFTER@LHC programme will allow one to further investigate the quark Sivers effect by measuring DY STSA $[297,298]$ over a wide range of $x^{\uparrow}\left(=x_{2}\right)$ and masses. With the high precision that AFTER@LHC will be able to achieve, one will accurately measure the Sivers function, if the sign change happens to be already established by the mentioned experiments. In case the asymmetry turns out to be small and these experiments cannot get to a clear answer, then AFTER@LHC will be able to confirm/falsify the sign change. Table 16 shows a compilation of the relevant parameters of future or planned polarised DY experiments. As can be seen, the AFTER@LHC programme offers the possibility to measure the DY $A_{N}$ in a broad kinematic range with an exceptional precision.

The DY measurement is the key to validate/falsify the Sivers effect for quarks. At AFTER@LHC, the target-rapidity range corresponds to a negative $x_{F}$ where the $A_{N}$ asymmetry is predicted to be large (Fig. 31) with large theoretical uncertainties. Fig. 31(a) shows the expected precision for DY $A_{N}$ measurement at AFTER@LHC for $\mathcal{L}=10 \mathrm{fb}^{-1}$ (which corresponds to one year of running), ${ }^{31}$ compared to two different theoretical predictions: AD'AM [298] and EIKV [294]. These two works performed fits of $A_{N}$ in SIDIS data, available for $x^{\uparrow} \lesssim 0.3$, using two different theoretical set-ups. The uncertainty band of AD'AM curve represents the statistical uncertainty of their fitted parameters after performing a variation of the total $\chi^{2}$ of about 20, while the one of EIKV is obtained by using the replica method (see e.g. Ref. [291]) with an effective variation of the total $\chi^{2}$ of about 1 ; this explains the difference of width among the curves. Thus the DY data at AFTER@LHC will put strict constraints on the Sivers effect for quarks, help to discriminate among different approaches, and accurately test one of the most important predictions of the TMD factorisation formalism, i.e. its sign change w.r.t. SIDIS. In addition, given that this effect can be framed as well within the CT3 approach, AFTER@LHC will obtain very useful data to constrain also the 3-parton correlation functions.

AFTER@LHC with a gas target offers also a unique opportunity for studies of STSA in polarised $p+{ }^{3} \mathrm{He}^{\uparrow}$ collisions. These have been studied at JLab Hall-A by several DIS/SIDIS experiments in the last two decades (see e.g. [307,308]), which the AFTER@LHC programme could then complement. Such reactions give access to polarised neutrons and thus to the Sivers functions in a neutron which can in principle shed some light on its isospin dependence. Let us also note that a polarised deuterium target can also provide a proxy to a polarised neutron target. Fig. 31(b) shows the statistical-uncertainty

\footnotetext{
30 Notice that another singled-out direction is needed, as the transverse momentum of the produced particles. See e.g. [285] for more details.

31 The statistical uncertainty $\delta$ on $A_{N}$ is calculated as $\delta_{A_{N}}=\frac{2}{\mathcal{P}_{\text {eff }}\left(\sigma^{\downarrow}+\sigma^{\uparrow}\right)^{2}} \sqrt{\left(\delta_{\sigma \uparrow} \sigma^{\downarrow}\right)^{2}+\left(\delta_{\sigma \downarrow} \sigma^{\uparrow}\right)^{2}}$, where $\delta_{\sigma}=\sqrt{\sigma+2 B}, \sigma$ is the cross section for a given configuration and $B$ is the background in that measurement. The yields are calculated at fixed $y_{\mu \mu}^{\text {lab. }}=[2.5,3.5,4.5]$, fixed $M_{\mu \mu}=$ $[4.5,5.5,6.5,7.5,8.5] \mathrm{GeV}$ and integrating over the transverse momentum of the lepton pair.
} 


\section{Table 16}

Compilation inspired by [1,27] of the relevant parameters for the future or planned polarised DY experiments. The effective polarisation $\left(\mathcal{P}_{\text {eff }}\right)$ is a beam polarisation (where relevant) or an average polarisation times a (possible) average dilution factor $(f\rangle$ (for a gas target, similar to the one developed for HERMES [102,299]) or a target polarisation times an average dilution factor $\langle f\rangle$ (for the targets used by COMPASS and E1039). For the AFTER@LHCb, AFTER@ALICE ${ }_{\mathrm{CB}}$ and AFTER@ALICE ${ }_{\mu}$ lines, the numbers correspond to a gas target with a storage cell (see Table 11 and Table 12) and $4<M_{\ell \ell}<9 \mathrm{GeV}$ (for the $x^{\uparrow}$ range). $\mathcal{F}$ is the (instantaneous) spin figure of merit of the set-up defined as $\mathcal{F}=\mathcal{L} \mathcal{P}_{\text {eff }}^{2} \sum_{i} A_{i}$, with $\mathcal{L}$ being the instantaneous luminosity. We stress that the values of $\mathcal{F}$ between different set-ups should be compared with care as it does not account for isospin and nuclear effects (via the variation of $f$ for instance) or acceptance effects neither for any energy or kinematical dependences of the DY production cross section which both alter the measured rates and the uncertainty of the asymmetry measurements. We refer to Section 3.2 for more details.

\begin{tabular}{|c|c|c|c|c|c|c|c|}
\hline Experiment & Colliding systems & Beam energy $[\mathrm{GeV}]$ & $\sqrt{s}[\mathrm{GeV}]$ & $x^{\uparrow}$ & $\mathcal{L}\left[\mathrm{cm}^{-2} \mathrm{~s}^{-1}\right]$ & $\mathcal{P}_{\text {eff }}$ & $\mathcal{F}\left[\mathrm{cm}^{-2} \mathrm{~s}^{-1}\right]$ \\
\hline $\begin{array}{l}\text { AFTER@LHCb: } z=0 \\
\text { AFTER@LHCb: } z=-0.4 \mathrm{~m} \\
\text { AFTER@LHCb: } z=-1.5 \mathrm{~m}\end{array}$ & $p \mathrm{H}^{\uparrow}$ & 7000 & 115 & $\begin{array}{l}0.05 \div 0.95 \\
0.02 \div 0.95 \\
0.01 \div 0.15\end{array}$ & $9.2 \times 10^{32}$ & $80 \%$ & $5.9 \times 10^{32}$ \\
\hline $\begin{array}{l}\text { AFTER@LHCb: } z=0 \\
\text { AFTER@LHCb: } z=-0.4 \mathrm{~m} \\
\text { AFTER@LHCb: } z=-1.5 \mathrm{~m}\end{array}$ & $p^{3} \mathrm{He}^{\uparrow}$ & 7000 & 115 & $\begin{array}{l}0.05 \div 0.95 \\
0.02 \div 0.95 \\
0.01 \div 0.15\end{array}$ & $1.3 \times 10^{33}$ & $23 \%$ & $2.1 \times 10^{32}$ \\
\hline $\begin{array}{l}\text { AFTER@LHCb: } z=0 \\
\text { AFTER@LHCb: } z=-0.4 \mathrm{~m} \\
\text { AFTER@LHCb: } z=-1.5 \mathrm{~m}\end{array}$ & $p D^{\uparrow}$ & 7000 & 115 & $\begin{array}{l}0.05 \div 0.95 \\
0.02 \div 0.95 \\
0.01 \div 0.15\end{array}$ & $5.6 \times 10^{32}$ & $78 \%$ & $6.8 \times 10^{32}$ \\
\hline $\begin{array}{l}\text { AFTER@ALICE }_{\mu}: z=0 \\
\text { AFTER@ALICE } \\
\text { AFTER@ALICE } \\
\text { AB }: z=-4.7 \mathrm{~m} \\
\text { ATER }\end{array}$ & $p \mathrm{H}^{\uparrow}$ & 7000 & 115 & $\begin{array}{l}0.10 \div 0.70 \\
0.08 \div 0.35 \\
0.40 \div 0.95\end{array}$ & $2.6 \times 10^{31}$ & $80 \%$ & $1.7 \times 10^{31}$ \\
\hline COMPASS (CERN) [300] & $\begin{array}{l}\pi^{-} \mathrm{NH}_{3}^{\uparrow} \\
\pi^{-}{ }^{6} \mathrm{LiD}\end{array}$ & 190 & 19 & $0.05 \div 0.55$ & $\begin{array}{l}2.0 \times 10^{32} \\
8.2 \times 10^{32}\end{array}$ & $\begin{array}{l}16 \% \\
22 \%\end{array}$ & $\begin{array}{l}8.7 \times 10^{31} \\
3.2 \times 10^{32}\end{array}$ \\
\hline PHENIX/STAR (RHIC) [301] & $p^{\uparrow} p^{\uparrow}$ & collider & 510 & $0.05 \div 0.10$ & $2.0 \times 10^{32}$ & $50 \%$ & $5.0 \times 10^{31}$ \\
\hline E1039 (FNAL) [302] & $\mathrm{pNH}_{3}^{\uparrow}$ & 120 & 15 & $0.10 \div 0.45$ & $3.9 \times 10^{34}$ & $15 \%$ & $1.5 \times 10^{34}$ \\
\hline E1027 (FNAL) [296] & $p^{\uparrow} \mathrm{H}_{2}$ & 120 & 15 & $0.35 \div 0.90$ & $1.0 \times 10^{35}$ & $60 \%$ & $7.2 \times 10^{34}$ \\
\hline NICA (JINR) [303] & $p^{\uparrow} p$ & collider & 26 & $0.10 \div 0.80$ & $1.0 \times 10^{32}$ & $70 \%$ & $4.9 \times 10^{31}$ \\
\hline fsPHENIX (RHIC) [304] & $p^{\uparrow} p^{\uparrow}$ & collider & 200 & $0.10 \div 0.50$ & $8.0 \times 10^{31}$ & $60 \%$ & $2.9 \times 10^{31}$ \\
\hline fsPHENIX (RHIC) [304] & $p^{\uparrow} p^{\uparrow}$ & collider & 510 & $0.05 \div 0.60$ & $6.0 \times 10^{32}$ & $50 \%$ & $1.5 \times 10^{32}$ \\
\hline PANDA (GSI) [305] & $\bar{p} H^{\uparrow}$ & 15 & 5.5 & $0.20 \div 0.40$ & $2.0 \times 10^{32}$ & $20 \%$ & $8.0 \times 10^{30}$ \\
\hline
\end{tabular}

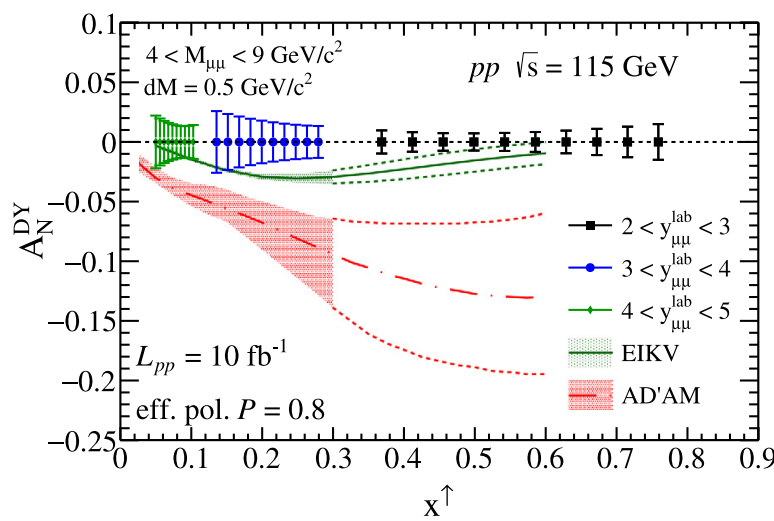

(a)

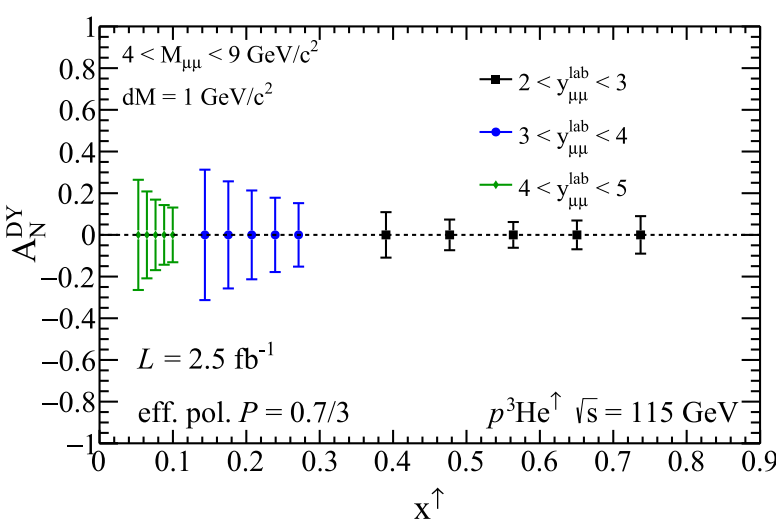

(b)

Fig. 31. (a) Two predictions (denoted AD'AM [298] and EIKV [294]) of the DY $A_{N}$ as a function of $x^{\uparrow}$ at AFTER@LHC, compared to the projected precision of the measurement [306]. The bands are filled in the region where the fits use existing SIDIS data, i.e. for $x^{\uparrow} \lesssim 0.3$, and hollow where they are extrapolations. (b) Similar projections for the DY $A_{N}$ as a function of $x^{\uparrow}$ in $p+{ }^{3} \mathrm{He}^{\uparrow}$ collisions at $\sqrt{s}=115 \mathrm{GeV}$ [306]. [In both cases, the bars show the statistical uncertainties for the quoted luminosities accounting for the background subtraction and polarisation-dilution effects].

predictions for DY measurements. In the case of ${ }^{3} \mathrm{He}^{\uparrow}$, a polarisation of $\mathrm{P}=70 \%$ can be achieved [102]. However, the effective polarisation, $\mathcal{P}_{\text {eff }}$, is diluted by a factor of 3 since only the neutron is polarised in the ${ }^{3} \mathrm{He}^{\uparrow}$. The projections for ${ }^{3} \mathrm{He}^{\uparrow}$ are prepared based on simulations for $p p$ collisions and applying corrections to account for change in signal and background yields. The combinatorial background is proportional to the number of binary nucleon-nucleon collisions $N_{\text {coll }}$, thus the background increases by a factor $N_{\text {coll }} \approx \sqrt{3}$ compared to $p p$. An additional isospin factor of $9 / 6$ for DY studies is included. The available integrated luminosity of $2.5 \mathrm{fb}^{-1}$ will allow for an exploratory measurement for DY production and precision study for quarkonium production (see Section 5.2.2). 
In addition, DY production with an unpolarised fixed-target will be extremely valuable to study the simplest TMD function at large $x$, namely the unpolarised TMD PDF [55,291,292,309-311]. A good knowledge of unpolarised TMDs is of fundamental importance in order to validate our understanding of their scale evolution and to reliably study azimuthal and spin asymmetries, as they always enter the denominators of these quantities.

Pion and kaon production. Pion and kaon STSAs have been extensively studied in the last three decades at Fermilab and BNL with hadron beams and at Jefferson Lab, CERN (COMPASS) and DESY (HERMES) with lepton beams (see e.g. [28-32,307,312,313]), observing large asymmetries in the valence region at large $x^{\uparrow}$, which motivated the introduction of the Sivers effect. As for now, similar studies have not been carried out with hadron beams on ${ }^{3} \mathrm{He}$, thus on a polarised neutron target, which however could give us original insights on the flavour symmetries of the correlation between the partonic transverse momentum and the nucleon spin. Along these lines, the AFTER@LHC programme relying on the LHCb and/or ALICE detectors, can play a crucial role.

Indeed, as shown in Fig. 32, the predicted $A_{N}$ for pion production with a neutron (a-b) and proton (c-d) target, based on the generalised parton model (GPM) approach (which is an extension of the parton model to include the transverse-momentum dependence $)^{32}$ and the CT3 approach, ${ }^{33}$ both indicate a "flavour" sign change.

From a pQCD point of view, pion and kaon production at AFTER@LHC can thus improve the current knowledge of the СT3 matrix elements involved in the production and fragmentation mechanisms [315], and help to clarify if the pion and kaon STSAs are really mainly generated by the Collins mechanism, as recently suggested [43].

$W^{ \pm}$boson production. So far, only the valence $u$ and $d$ quark Sivers functions have been constrained, while sea-quark Sivers functions remain largely unknown [294]. In this sense, STSAs for vector-boson production offer a complementary tool to STSAs for DY production, giving access to the flavour dependence of the Sivers function. Moreover, they can also serve to test the sign change of the Sivers effect with respect to SIDIS.

As explained in Section 5.1.1, roughly 250 (off-shell) $W^{+}$and 60 (off-shell) $W^{-}$per year are expected to be collected with a luminosity of $10 \mathrm{fb}^{-1}$. These yields would allow one to achieve a statistical uncertainty for $A_{N}$ of roughly $0.1-0.2 .^{34}$ This uncertainty is comparable to the precision of the $A_{N}$ measurement for $W^{ \pm} \rightarrow l^{ \pm} v$ in $p^{\uparrow}+p$ production at RHIC [79]. In 2017 the STAR experiment carried out a measurement of the transverse single-spin asymmetry in $p^{\uparrow}+p \rightarrow W^{ \pm} / Z^{0}$ aiming at a statistical precision on the level of 5\% [80]. The AFTER@LHC $W^{ \pm}$measurement will cover a different range in $x^{\uparrow}$. As such, measurements at AFTER@LHC and RHIC will be complementary.

In general, both transverse and longitudinal polarisations of a gas target are feasible, as it was demonstrated by the HERMES experiment. In that case, a set-up with longitudinal polarisation target was used in 1996-2000 and then changed to a transverse one in 2001 [126]. Therefore, we finally comment on the possibility to constrain the sea-quark-helicity distributions at AFTER@LHC by measuring the spin asymmetries for a longitudinally polarised target. At the leading order in $\alpha_{s}$, the asymmetries for $W^{+}$and $W^{-}$bosons are simply related to the (helicity) PDFs as

$$
A_{U L}^{W^{+}} \propto \frac{g_{1}^{u}\left(x_{1}\right) f_{1}^{\bar{d}}\left(x_{2}\right)-g_{1}^{\bar{d}}\left(x_{1}\right) f_{1}^{u}\left(x_{2}\right)}{f_{1}^{u}\left(x_{1}\right) f_{1}^{\bar{d}}\left(x_{2}\right)+f_{1}^{\bar{d}}\left(x_{1}\right) f_{1}^{u}\left(x_{2}\right)}, \quad A_{U L}^{W^{-}} \propto \frac{g_{1}^{d}\left(x_{1}\right) f_{1}^{\bar{u}}\left(x_{2}\right)-g_{1}^{\bar{u}}\left(x_{1}\right) f_{1}^{d}\left(x_{2}\right)}{f_{1}^{d}\left(x_{1}\right) f_{1}^{\bar{u}}\left(x_{2}\right)+f_{1}^{\bar{u}}\left(x_{1}\right) f_{1}^{d}\left(x_{2}\right)},
$$

where $g_{1}^{q}$ is the helicity PDF of the quark $q$. Dedicated simulations are however needed to quantify such constraints.

Direct-photon production. The quark Sivers effect can also be studied via direct-photon production STSAs. Fig. 33 shows the expected $A_{N}$ as a function of the photon $p_{T}$ for $x_{F}=-0.2$ (i.e. in a range accessible by LHCb) for both aforementioned approaches. Contrary to the DY and $\pi$ production cases, the predicted signs of $A_{N}$ differ. This is related to the sign "mismatch" issue (see e.g. [316]). Even though the magnitude of $A_{N}$ is the largest at low $p_{T}(<1 \mathrm{GeV})$ where the background is probably very challenging and the application of pQCD questionable, measurements with a precision on the order of $1 \%$ for $x_{F}=-0.2$ should however be sufficient to discriminate between the predictions of both approaches for $p_{T}$ above $5 \mathrm{GeV}$.

By measuring $A_{N}$ for quark-induced processes which do not involve fragmentation, in particular prompt photons (compared to charged and neutral pions and kaons), the AFTER@LHC programme will allow one to distinguish the Sivers and Collins mechanisms [27,317], and in turn constrain the quark contribution to the transverse spin of the proton, along with the constraints from DY production data.

\subsubsection{Gluon Sivers effect}

DY production is the golden process to access the intrinsic transverse motion of quarks in a nucleon. However, there is no analogous process to probe the gluon content, which is both experimentally clean and theoretically well-controlled.

\footnotetext{
32 The isospin symmetry is used to implement the neutron Sivers functions by using the extracted proton ones in Ref. [298]: $f_{1 T}^{\perp u / \text { neutron }}=f_{1 T}^{\perp d / p r o t o n}$ and $f_{1 T}^{\perp d / \text { neutron }}=f_{1 T}^{\perp u / p r o t o n}$. This is supported by the STSA on deuteron target measured by COMPASS [32,314], well compatible with zero. These predictions include both Sivers and Collins contributions, which are added together in the estimates of the central values as well as of the statistical uncertainty bands.

33 The isospin symmetry is used again to implement the neutron twist-3 matrix elements by using the extracted proton ones in Ref. [43].

34 The uncertainty is calculated in the same way as for DY $A_{N}$, and we assumed that the background is negligible.
} 


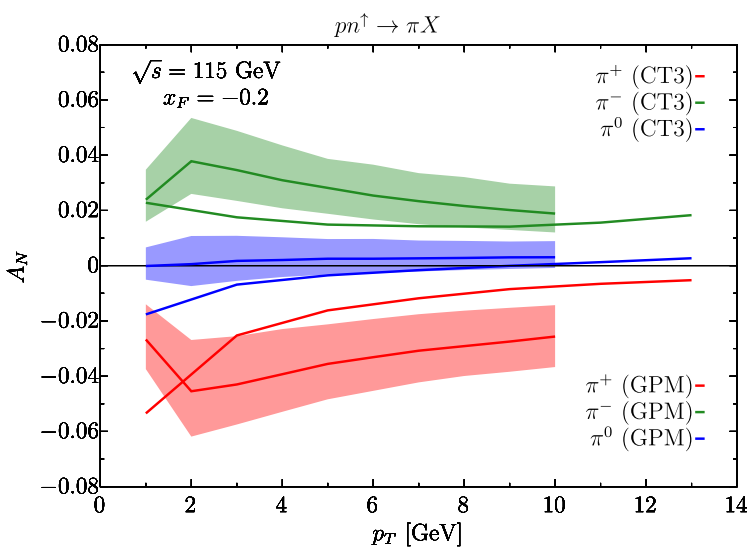

(a)

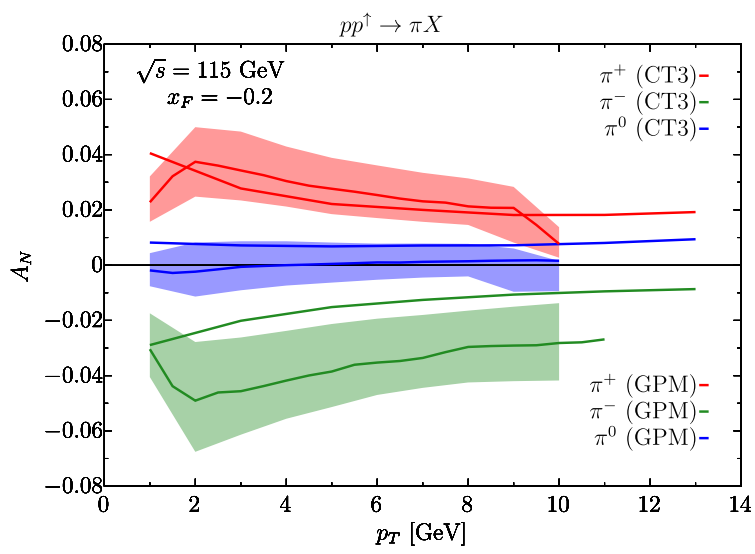

(c)

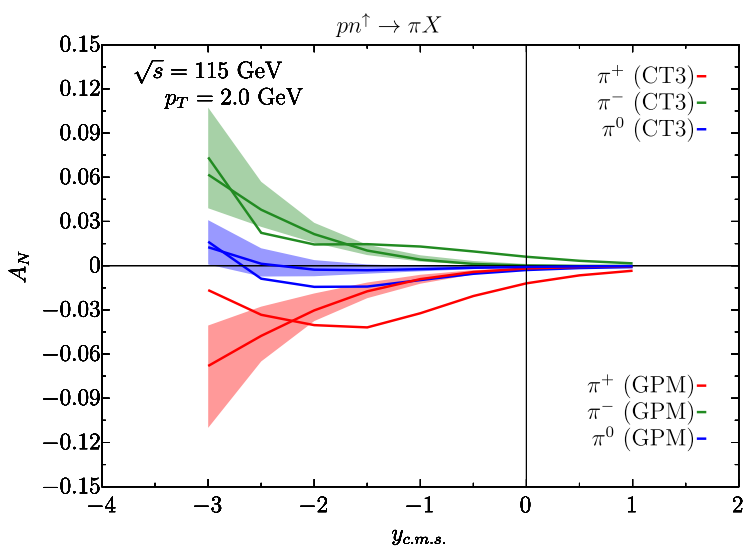

(b)

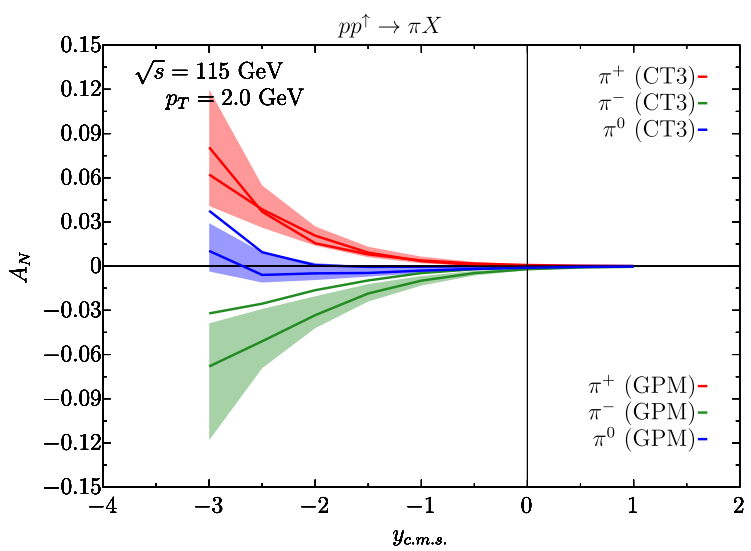

(d)

Fig. 32. Predicted $A_{N}$ for pion production as a function of (a) transverse momentum $p_{T}$ and (b) rapidity $y$, in the AFTER@LHC kinematics for a neutron target (accessible with ${ }^{3} \mathrm{He}^{\uparrow}$ ), based on both GPM and CT3 formalisms. (c-d): same as (a-b) but for a proton target.

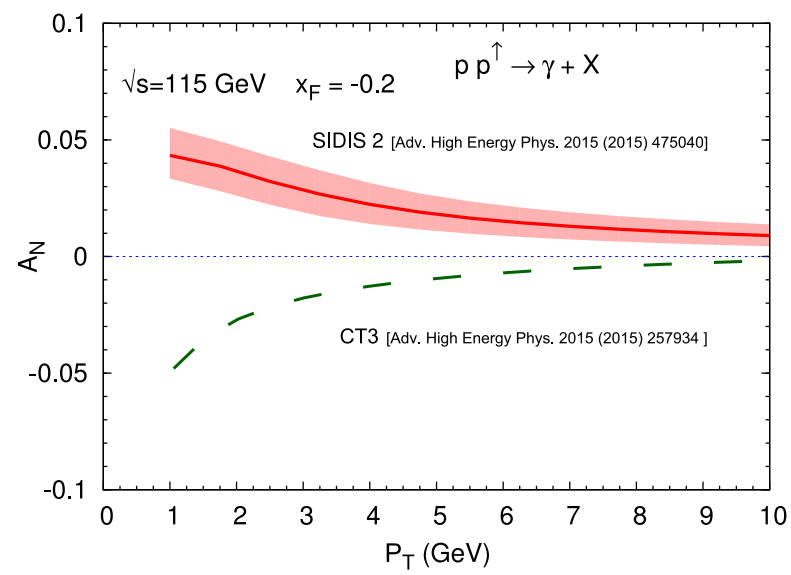

Fig. 33. Prediction for the direct-photon $A_{N}$ as a function of $p_{T}[298,315]$ for $x_{F}=-0.2$. No theoretical uncertainties are shown for the CT3 prediction.

For instance, $H^{0}$ boson production serve as such a process, since it is a gluon-gluon fusion process into an observed colour singlet, but it is experimentally demanding in terms of luminosity and energy. 


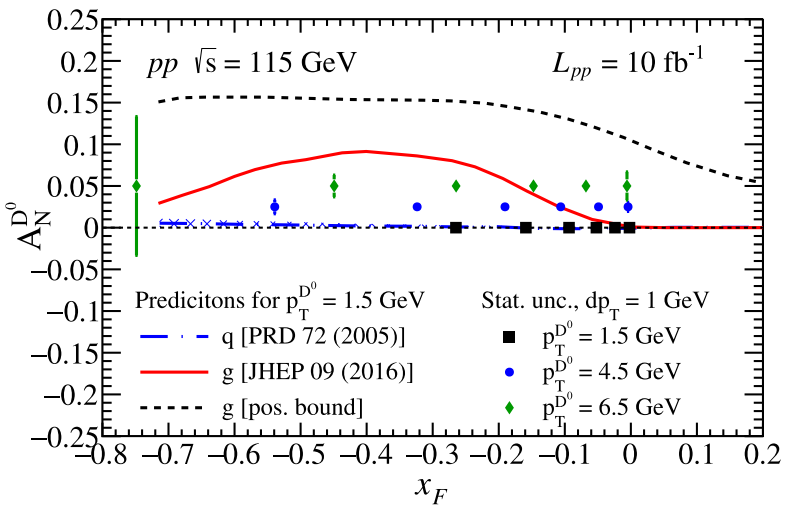

(a)

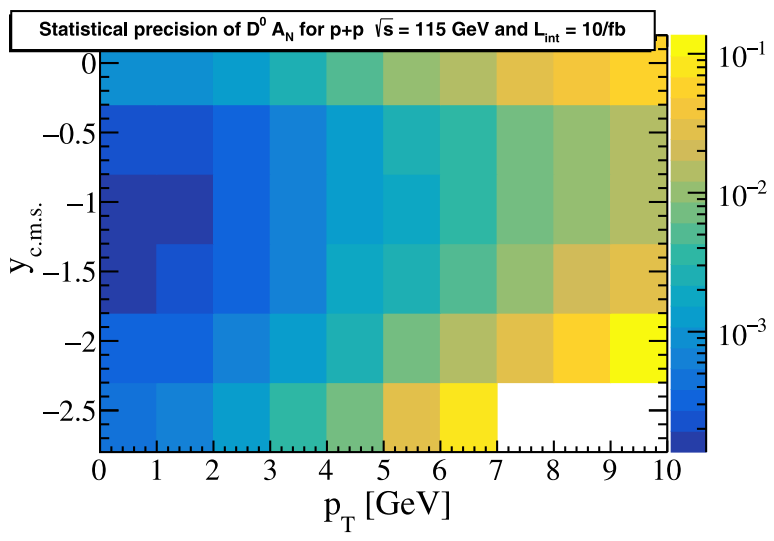

(b)

Fig. 34. Projections for the statistical precision of open charm $A_{N}$ as a function of (a) $x_{F}$ at fixed $p_{T}$ and of (b) $y_{\text {c.m.s. }} \& p_{T}$, at $\sqrt{s_{N N}}=115 \mathrm{GeV}$ and 10 $\mathrm{fb}^{-1}$ of luminosity using a LHCb-like detector. The $D^{0}$ yields are taken from FONLL $[325,326]$ calculations within the LHCb acceptance; the efficiency and the S/B ratio are extrapolated from [117]. For most of the points, statistical uncertainties are smaller than the marker size. Estimates in (a) for different $p_{T}$ bins were made assuming bin width $d p_{T}=1 \mathrm{GeV}$, and were displaced vertically for a better visibility. The theory curves represent the SIDIS predictions for quarks and gluons, along with the positivity upper bound of the gluon Sivers effect [327,328].

Currently, one of the best tools at our disposal is the production of quarkonia. In fact, a major strength of AFTER@LHC is the large production rates for open heavy-flavour mesons and quarkonium states. The expected rates for a single year of data taking are roughly $10^{6} \Upsilon$ and $10^{9} \mathrm{~J} / \psi$. In addition, these processes are mainly sensitive to the gluon content of the colliding hadrons. They are very useful probes to precisely access and constrain the gluon Sivers effect, which is essentially unknown [63,318]

Moreover, due to the inherent process dependence of the TMDs, different processes will probe different gluon TMD functions [75,319]. In fact, the generalised universality of gluon TMDs is more involved than that of the quark TMDs, due to the richer gauge-link structure in their operator definition. In particular, in the case of the gluon Sivers function, it is known that all the functions that can be probed in different processes can be reduced to only two independent ones [75]. The AFTER@LHC programme offers a unique possibility to test all these theoretical predictions, either confirming them or quantifying the potential discrepancies, if any.

Open heavy-flavour production. The $A_{N}$ for open heavy-flavour production gives access to the gluon Sivers effect (see e.g. [320,321]). It also offers the possibility to study the process dependence of $A_{N}$, by measuring charm quarks and anti-quarks separately [322], being a unique probe of the C-parity odd twist-3 tri-gluon correlator [323,324].

Fig. 34 shows the estimated statistical precision for charmed-meson $A_{N}$ at backward and mid-rapidity (in the c.m.s.). As can be seen, even in the case of a moderate target polarisation we would expect a very precise measurement for $p_{T} \lesssim 5 \mathrm{GeV}$. Such measurements can only be performed at AFTER@LHC. It can definitely be achieved in the $K \pi$ decay channel, and possibly in the lepton-decay channel, despite of the presence of many sources for the background (see e.g. [329]).

Fig. 35 shows the estimated statistical precision for open bottom $A_{N}$, measured via non-prompt $J / \psi$, as a function of $p_{T}$ at mid-rapidity, which is similar to that of the prompt charmonium at the sub-percent level. It is thus clear that such a measurement would open a new era of precision studies of STSAs for open heavy-flavour production.

Vector quarkonium production. Fig. 36(a) shows the estimated uncertainties at AFTER@LHC for the $\Upsilon A_{N}$ as a function of $x_{F}$ in $p p$ collisions at $\sqrt{s}=115 \mathrm{GeV}$, while Fig. 36(b) shows the projected uncertainties for $J / \psi$ compared to the already existing measurements at $\sqrt{s}=200 \mathrm{GeV}[33,66]$. With the expected charmonium yields [331] these results are only limited by the systematic uncertainties, and can thus give a very precise access to the gluon content of the proton over a much wider $x$ range than at RHIC. As for now, the experimental measurements are compatible with zero, which could be due to the following reasons: first, the gluon Sivers function might be zero; second, the effect of the Sivers function might disappear when integrated over $p_{T}$, in case it has a node; third, the dominant $J / \psi$ production mechanism might be via colour-octet transitions, whose STSA in $p p$ collisions is thought to vanish as compared to the STSA for colour-singlet transitions [332]. However, we note that the arguments in support of the latter reason are derived at leading-twist, and thus deviations on the order of $10 \div 20 \%$ of the measured asymmetry should certainly not be excluded. On the other hand, the generation of such a STSA through a Collins-like fragmentation mechanism has not been discussed in the literature. In any case, the precise measurements performed within the AFTER@LHC programme can give us a handle to discriminate the discussed scenarios.

Fig. 37 shows the expected statistical precision for $J / \psi A_{N}$ with an ALICE-like detector for $p p^{\uparrow}$ collisions with $45 \mathrm{pb}^{-1}$ of luminosity. The expected $J / \psi$ and the background yields were extrapolated from the $J / \psi$-rapidity spectrum and the 


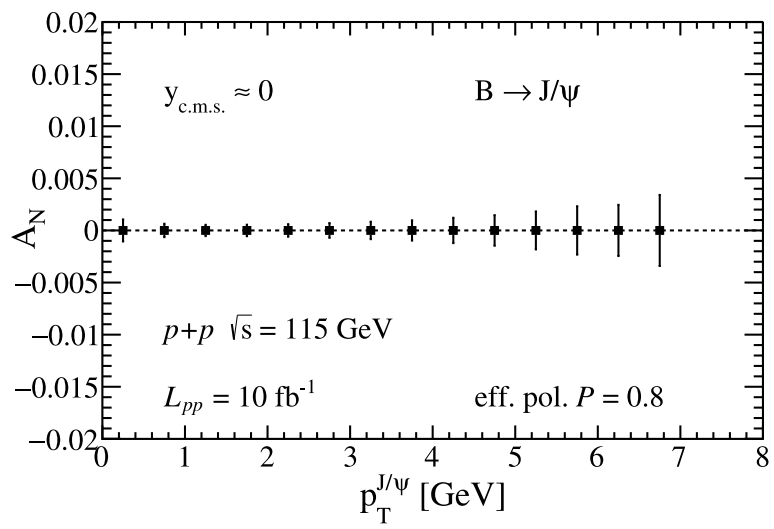

Fig. 35. Projections for the statistical precision of open bottom $A_{N}$ (measured via $B \rightarrow J / \psi$ ) as a function of $p_{T}$ at $y_{\text {c.m.s. }} \approx 0$ and $\sqrt{s_{N N}}=115$ GeV measured with a LHCb-like detector with integrated luminosity of $10 \mathrm{fb}^{-1}$. The $B \rightarrow J / \psi$ yields are calculated based on the FONLL predictions for the bottom-quark yields, and the $B \rightarrow J / \psi$ fragmentation is calculated using PYTHIA. The calculations include the acceptance and reconstruction efficiency of a LHCb-like detector. See [330] for more details.

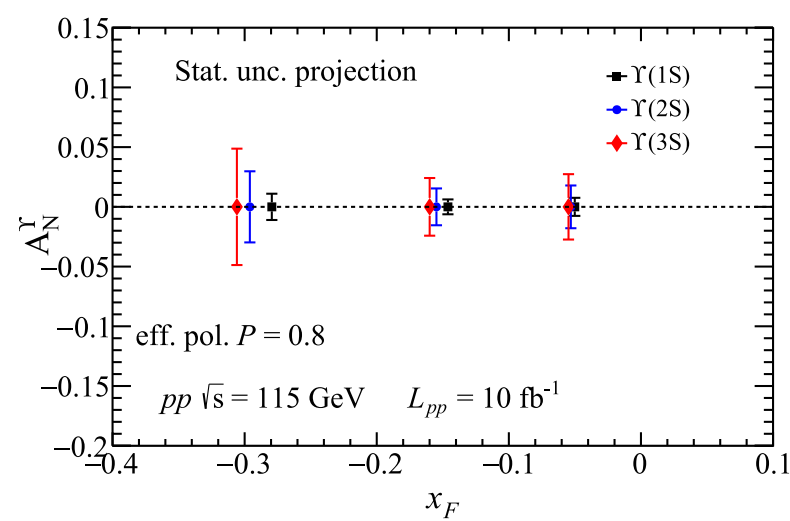

(a)

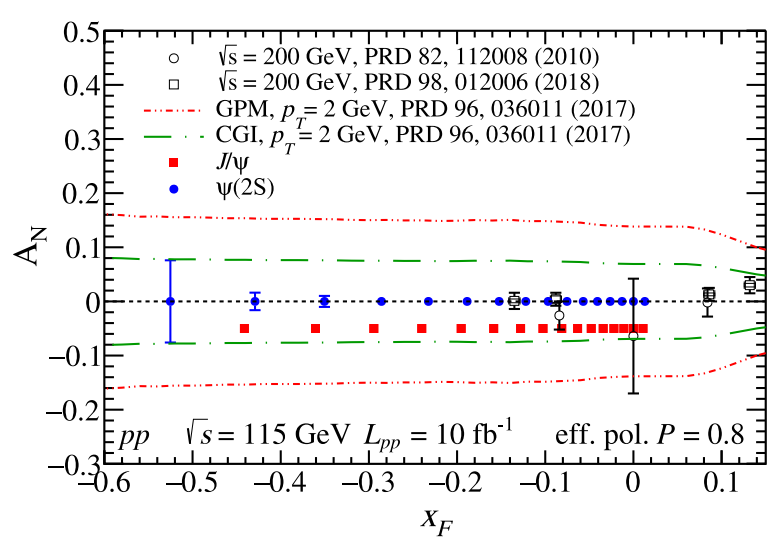

(b)

Fig. 36. Statistical-precision projections for (a) $\Upsilon(n S)$ and (b) $\psi(n S) A_{N}$ as a function of $x_{F}$ compared to the existing measurements [33,66] and predictions by the generalised parton model (GPM) and the colour gauge invariant (CGI) version of the GPM model [321]. The quarkonium states are assumed to be measured in a di-muon channel with a LHCb-like detector. The signal and the background are calculated in realistic simulations that take into account the performance of the LHCb detector [306,331]. [(a): Adapted from [331].].

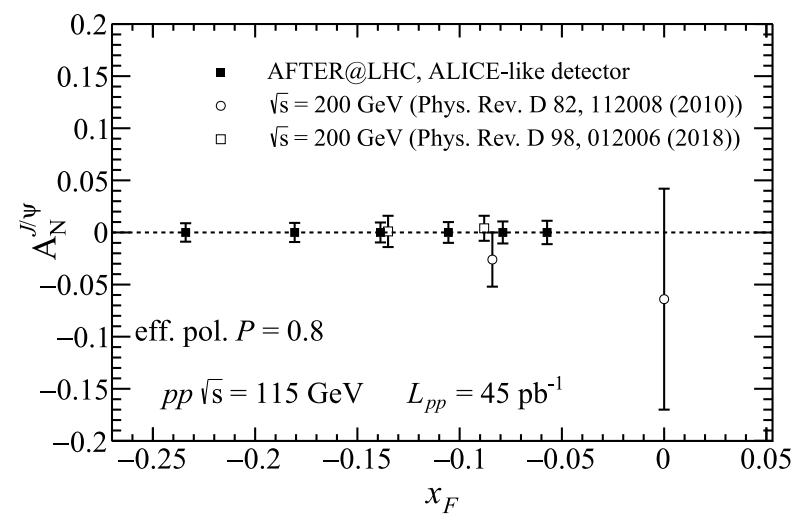

Fig. 37. Statistical-precision projections for $J / \psi A_{N}$ as a function of $x_{F}$ compared to the existing measurements $[33,66]$ for AFTER@ALICE ${ }_{\mu}$ with target located at the nominal IP $\left(z_{\text {target }} \approx 0\right)$. The $J / \psi$ di-muon spectrum is assumed to be measured in the Muon Spectrometer of the ALICE detector. The signal and the background are extrapolated at $\sqrt{S_{N N}}=115 \mathrm{GeV}$ from the ALICE measurements in [333]. 


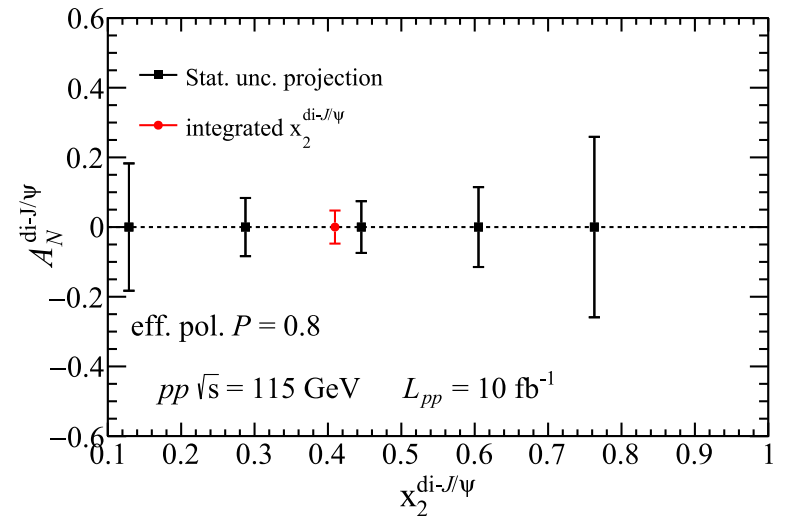

(a)

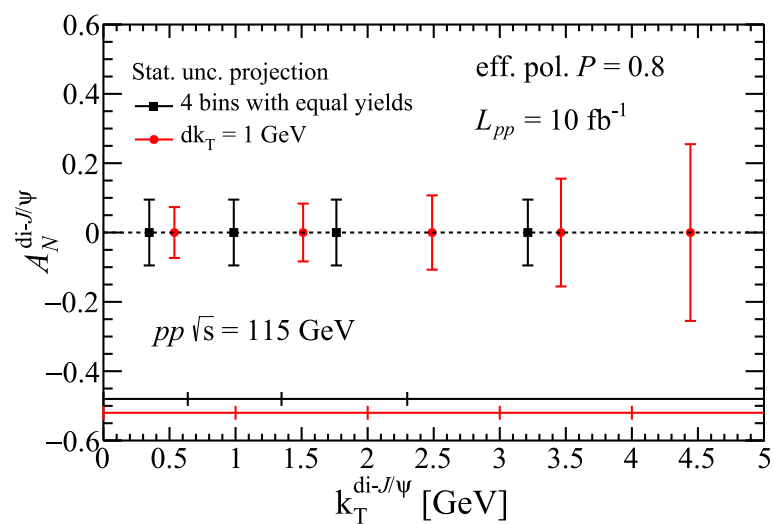

(b)

Fig. 38. Statistical-precision projections for di-J/ $\psi A_{N}$ as a function of (a) the pair $x_{2}$ and (b) the pair $k_{T}$ with a LHCb-like detector. The horizontal lines in (b) denote the widths of the $k_{T}$ bins used for the calculations. [Adapted from [331].].

signal-to-background ratios of [333] with the procedure described in [306]. The signal-to-background ratio at $115 \mathrm{GeV}$ is 1.2 and an efficiency of $13 \%$ was assumed [334].

C-even quarkonium production. The production of $C$-even states can fruitfully be investigated $[70,71,335,336]$. With a LHCb-like detector, ${ }^{35}$ STSA measurements for $\chi_{c}, \chi_{b}$ and $\eta_{c}$ are possible as demonstrated by studies of various $\chi_{c}$ states [337,338] in the busier collider environment down to a transverse momentum $p_{T}$ as low as $2 \mathrm{GeV}$. LHCb allowed for the first study of inclusive $\eta_{c}$ production [339] above $p_{T}=6 \mathrm{GeV}$ as well as non-prompt $\eta_{c}(2 S)$ [340]. Prompt studies are definitely within the LHCb reach [341]. At lower energies, the reduced combinatorial background will definitely allow one to access lower $p_{T}$ with very reasonable statistics. Indeed, the cross section for pseudoscalar-charmonium production should be similar to that of the vector ones. The main differences in the expected yields come from likely smaller branchings (with the notable exception of the $K K \pi$ decay) and detection efficiencies. Measuring the STSA of $\eta_{c}$ also gives a clean access to tri-gluon correlation functions [342] but also, if one can measure its $p_{T}$ dependence, to the transverse momentum dependence of the gluon Sivers function relevant for hadron-induced reactions.

Associated production. Associated-production channels [71,72,343-348] are fundamental tools to access the gluon Sivers effect and also probe the gluon TMD sector in general. Even if the production rates are lower than for single production, they would allow one to probe the TMD evolution mechanism $[288,336]$ by tuning the mass of the final state.

A few different processes are potentially interesting in this context, for instance $J / \psi-J / \psi, J / \psi-\gamma, \gamma-\gamma, \Upsilon(n S)-\gamma$. The $J / \psi-J / \psi$ production seems to be the most practical one since the yields are not too small [225] and the measurement is relatively straightforward (compared, for instance, to direct $\gamma$ studies). Fig. 38 shows the $A_{N}$ for double $J / \psi$ production as a function of the transverse momentum of the pair, $k_{T}$, and the corresponding $x_{2}$. We consider two scenarios for the analysis of $A_{N}$ as a function of $k_{T}$ : bins with a fixed width of $1 \mathrm{GeV}\left(d k_{T}=1 \mathrm{GeV}\right.$, red points) and bins with equal number of yields. Here, we model the $k_{T}$ dependence as a Gaussian distribution with the width $\sigma=2 \mathrm{GeV}$. The $x_{2}$-integrated $A_{N}$ will allow for the determination of the STSA with a few percent precision, and the $A_{N}\left(k_{T}\right)$ gives access - for the first time - to the $k_{T}$ dependence of the gluon Sivers TMD up to $k_{T} \approx 4 \mathrm{GeV}$.

\subsubsection{Quark-induced azimuthal asymmetries}

In Section 5.2.1 we discussed the extraction of the Sivers asymmetry from the DY production cross-section. However this process can also give valuable information on other asymmetries, and thus on other TMDs. In fact, the cross-section for a transversely polarised target (and an unpolarised beam) can be schematically written in terms of the following structure functions [349]:

$$
\begin{aligned}
A_{U U}^{\cos 2 \phi} & \sim \frac{h_{1}^{\perp q}\left(x_{1}, k_{1 T}^{2}\right) \otimes h_{1}^{\perp \bar{q}}\left(x_{2}, k_{2 T}^{2}\right)}{f_{1}^{q}\left(x_{1}, k_{1 T}^{2}\right) \otimes f_{1}^{\bar{q}}\left(x_{2}, k_{2 T}^{2}\right)}, \\
A_{U T}^{\sin \phi_{S}} & \sim \frac{f_{1}^{q}\left(x_{1}, k_{1 T}^{2}\right) \otimes f_{1 T}^{\perp \bar{q}}\left(x_{2}, k_{2 T}^{2}\right)}{f_{1}^{q}\left(x_{1}, k_{1 T}^{2}\right) \otimes f_{1}^{\bar{q}}\left(x_{2}, k_{2 T}^{2}\right)}, \\
A_{U T}^{\sin \left(2 \phi+\phi_{S}\right)} & \sim \frac{h_{1}^{\perp q}\left(x_{1}, k_{1 T}^{2}\right) \otimes h_{1 T}^{\perp \bar{q}}\left(x_{2}, k_{2 T}^{2}\right)}{f_{1}^{q}\left(x_{1}, k_{1 T}^{2}\right) \otimes f_{1}^{\bar{q}}\left(x_{2}, k_{2 T}^{2}\right)},
\end{aligned}
$$

\footnotetext{
35 That is, assuming a detector that has a good momentum and energy resolution for muons, a decent energy resolution for photons, PID for protons and anti-protons and excellent vertexing capabilities.
} 


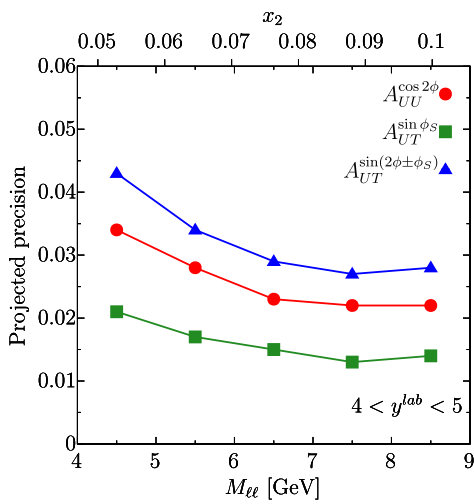

(a)

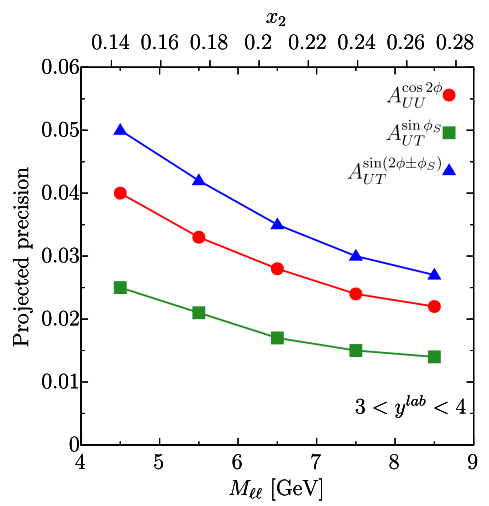

(b)

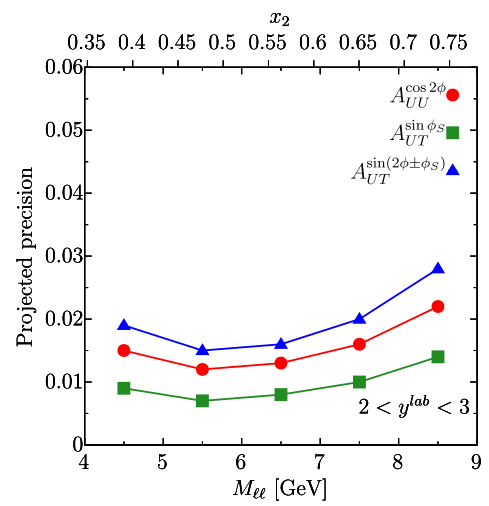

(c)

Fig. 39. Expected statistical uncertainty on asymmetries in DY production at AFTER@LHCb, computed all for $\mathcal{L}_{p p}=10$ fb ${ }^{-1}$ and $\mathcal{P}_{\text {eff. }}=0.8$. The rapidity has been integrated over the bins specified in the plots, as well as the mass in bins of $d M=1 \mathrm{GeV}$. [The statistical uncertainties are calculated using following expressions: $\delta\left(A_{U T}^{\sin \phi_{S}}\right)=1 / \mathcal{P}_{\text {eff. }} \times \sqrt{2} / \sqrt{S+2 B}, \delta\left(A_{U U}^{\cos 2 \phi_{S}}\right)=2 \sqrt{2} / \sqrt{S+2 B}$ and $\delta\left(A_{U T}^{\sin \left(2 \phi \pm \phi_{S}\right)}\right)=2 / \mathcal{P}_{\text {eff. }} \times \sqrt{2} / \sqrt{S+2 B}$, where $S$ is the signal yield, $B$ is the background yield and $\mathcal{P}_{\text {eff. }}$ is the effective polarisation in a given measurement.]

$$
A_{U T}^{\sin \left(2 \phi-\phi_{S}\right)} \sim \frac{h_{1}^{\perp q}\left(x_{1}, k_{1 T}^{2}\right) \otimes h_{1}^{\bar{q}}\left(x_{2}, k_{2 T}^{2}\right)}{f_{1}^{q}\left(x_{1}, k_{1 T}^{2}\right) \otimes f_{1}^{\bar{q}}\left(x_{2}, k_{2 T}^{2}\right)},
$$

where $h_{1}^{q}$ is the transversity, $h_{1}^{\perp q}$ the Boer-Mulders function and $h_{1 T}^{\perp q}$ the pretzelosity $\left(f_{1}^{q}\right.$ and $f_{1 T}^{\perp q}$ are the already introduced unpolarised TMD PDF and the Sivers function, respectively). Again $\otimes$ stands for a convolution in momentum space, and a sum over parton flavours is understood. The superscript on the A's means that we weight the cross-section with that angular term to single out the corresponding angular modulation.

Let us focus on the Boer-Mulders function $h_{1}^{\perp}$, which encodes the correlation between the quark transverse spin and its transverse momentum, namely it represents a spin-orbit effect for the quark inside an unpolarised proton. This function, like the quark Sivers function, is naive time-reversal odd (T-odd), and thus it changes sign under time-reversal transformations. ${ }^{36}$ In particular, a sign change is predicted for $h_{1}^{\perp}$ probed in SIDIS and DY production. Moreover, it might help explain [69] the violation of the Lam-Tung relation in unpolarised DY reaction [68]. Hints about the transverse momentum dependence of the Boer-Mulders function $h_{1}^{\perp}$ have been extracted from SIDIS data in [350]. AFTER@LHC will contribute to the study of the Boer-Mulders function in DY production, shedding light on its process dependence and on the TMD formalism in general.

In Fig. 39 we show the expected precision achievable at AFTER@LHC for different angular modulations of the DY production cross-section in different kinematic regions (rapidity, invariant mass, momentum fraction in the (un)polarised target nucleon). We note that $A_{U U}^{\cos 2 \phi}$ could be measured without a polarised target and that asymmetries with faster modulations are usually determined with a poorer precision.

\subsubsection{Gluon-induced azimuthal asymmetries}

In the quark case, there are two leading-twist TMDs, as we have discussed, the unpolarised $f_{1}^{q}\left(x, k_{T}^{2}\right)$ and the BoerMulders $h_{1}^{\perp q}\left(x, k_{T}^{2}\right)$ functions. For a gluon in an unpolarised proton, the relevant functions are the unpolarised distribution $f_{1}^{g}\left(x, k_{T}^{2}\right)$ and the distribution of linearly polarised gluons $h_{1}^{\perp g}\left(x, k_{T}^{2}\right)[53,54]$.

The phenomenology of $h_{1}^{\perp g}$ is potentially easier than that for the Boer-Mulders function in the quark case, because it is T-even and matched onto the twist- 2 unpolarised collinear distributions $f_{1}^{g, q}$, whereas $h_{1}^{\perp q}$ is matched onto the twist-3 collinear matrix elements, which are so far unknown. However, no experimental extractions of $h_{1}^{\perp g}$ have been performed yet. Recently, it has been proposed to access both $f_{1}^{g}$ and $h_{1}^{\perp g}$ in $\mathrm{di}-J / \psi$ and $\Upsilon$ production in hadronic collisions [348,351], for which data with sensitivity to transverse momenta have been collected at the LHC. It is expected that $h_{1}^{\perp g}$ reaches its maximal size in the small- $x$ regime [54,352-354]. Its role in different $x$-regions has yet to be explored. Factorisation proofs have recently been provided for $\eta_{c, b}$ production [355,356]. It is also expected to be constrained from azimuthal-asymmetry measurements at the future EIC and the LHeC [319,357], and also possibly from measurements at RHIC and the LHC [343].

The impact of linearly polarised gluons in $H^{0}$ production has been addressed e.g. in [336,358-360]. Their effect has been predicted for gluon fusion into two photons in [343,361], for (pseudo)scalar quarkonium production in [70,71], for vector quarkonium production in [362,363] and for $H^{0}$ plus jet production in [344]. Associated production of quarkonium and $Z$

\footnotetext{
36 Naive time reversal stands for time reversal but without the interchange of initial and final states [48].
} 


\section{Table 17}

Expected $q_{T}$ modulations generated by $h_{1}^{\perp g}$ for a selection of quarkonium-production observables, along with the expected yields and $x_{2}$ ranges derived from $x_{2}=M e^{y_{\text {c.m.s. }}} / \sqrt{s}$ for a rapidity coverage $-2.8<y_{\text {c.m.s. }}<0.2$ and $\sqrt{s}=115 \mathrm{GeV}$. The modulation expectations are meant to approximately account for TMD evolution effects [71].

\begin{tabular}{lllll}
\hline Process & Expected yield & $\chi_{2}$ range & $M[\mathrm{GeV}]$ & $q_{T}$ modulation \\
\hline$\eta_{c}[70,71]$ & $\mathcal{O}\left(10^{6}\right)$ & $0.02 \div 0.5$ & $\mathcal{O}(3)$ & $0 \div 80 \%$ \\
$\chi_{c 0}(1 P)[70]$ & $\mathcal{O}\left(10^{4}\right)$ & $0.02 \div 0.5$ & $\mathcal{O}(3)$ & $0 \div 80 \%$ \\
$\chi_{c 2}(1 P)[70]$ & $\mathcal{O}\left(10^{6}\right)$ & $0.02 \div 0.5$ & $\mathcal{O}(3)$ & $<1 \%$ \\
$\chi_{b 0}(n P)[70]$ & $\mathcal{O}\left(10^{2}\right)$ & $0.1 \div 1$ & $\mathcal{O}(10)$ & $0 \div 60 \%$ \\
$\chi_{b 2}(n P)[70]$ & $\mathcal{O}\left(10^{3}\right)$ & $0.1 \div 1$ & $\mathcal{O}(10)$ & $<1 \%$ \\
\hline
\end{tabular}

\section{Table 18}

Expected azimuthal asymmetries generated by $h_{1}^{\perp g}$ for a selection of quarkonium-associated-production observables, along with the expected yields and $x_{2}$ ranges derived from $x_{2}=M e^{Y_{\text {c.m.s. }}} / \sqrt{s}$ for a rapidity coverage $-2.8<Y_{\text {c.m.s. }}<0.2$ and $\sqrt{s}=115 \mathrm{GeV}$ ( $Y_{\text {c.m.s. }}$ refers to the rapidity of the observed 2-particle system). The modulation expectations are meant to approximately account for TMD evolution effects [348].

\begin{tabular}{llllll}
\hline Process & Expected yield & $x_{2}$ range & $M[\mathrm{GeV}]$ & $\cos 2 \phi$ modulation & cos $4 \phi$ modulation \\
\hline$J / \psi+\gamma[72]$ & $1000 \div 2000$ & $0.1 \div 0.6$ & $\mathcal{O}(10)$ & $0 \div 5 \%$ & $0 \div 2 \%$ \\
$J / \psi+J / \psi[351]$ & $300 \div 1500$ & $0.1 \div 0.8$ & $8 \div 12$ & $0 \div 8 \%$ & $0 \div 20 \%$ \\
\hline
\end{tabular}

boson has been investigated in [364]. Associated production of quarkonium plus one photon [72] is also promising, due to the possibility of producing final states with different invariant masses, suited thus to be analysed using TMD factorisation and to test TMD evolution. This process, together with $\eta_{b, c}$ production [70,71,365] and double $J / \psi$ production [225], can be investigated within the AFTER@LHC programme.

Several processes can be measured at the proposed AFTER@LHC programme in order to constrain $h_{1}^{\perp g}$ in yet unexplored kinematic regions. In Table 17 we show those in which the effect of the presence of $h_{1}^{\perp g}$ is the modulation of the transverse-momentum spectrum, referred to as " $q_{T}$ modulation", while in Table 18 we show those for which $h_{1}^{\perp g}$ creates an azimuthal modulation of the spectrum, referred to as "cos $n \phi$ modulation". We notice that in all the mentioned processes the same $h_{1}^{\perp g}$ function is probed, since the gauge-link structure is the same. As can be seen, overall the AFTER@LHC programme offers a great opportunity to constrain $h_{1}^{\perp g}$ through all these processes.

At AFTER@LHC, it will be possible to study the potential TMD factorisation breaking effects [366] in the production of $\chi_{c 0}$ and $\chi_{c 2}$ [70]. Moreover, $\eta_{c}$ production at low transverse momentum [355] will be accessed, complementing the high transverse momentum region measured by LHCb and going beyond RHIC's capabilities.

As already mentioned for the case of quark TMDs, the AFTER@LHC programme can be useful also to better constrain the simplest of the gluon TMDs, i.e. the unpolarised gluon TMD PDF, which remains so far unconstrained (see however a first attempt in [351]). Like its quark counterpart, it enters the denominators of all spin and azimuthal asymmetries, and thus its knowledge is fundamental in order to reliably study any TMD-related asymmetry.

\subsubsection{From TMD PDFs to the partonic orbital angular momentum}

On top of providing a handle on the intrinsic spin of partons, the TMD formalism intuitively connects with the orbital angular momentum of the quarks through the correlations proportional to the partonic transverse momenta. In fact, conservation of total angular momentum imposes that off-diagonal TMDs (i.e. those with $\Delta \lambda=\left(\Lambda^{\prime}-\Lambda\right)-\left(\lambda^{\prime}-\lambda\right) \neq 0$, where $\Lambda^{(\prime)}$ and $\lambda^{(\prime)}$ are the initial (final) target and parton light-front helicities) would vanish in the absence of the orbital angular momentum (OAM). Some of these off-diagonal TMDs appear to be experimentally sizeable [31,32,367], confirming henceforth the presence of a significant amount of OAM inside the nucleon. This rises the question as to whether TMDs can be used to quantify more precisely the OAM.

It has been observed within many effective quark models that the expectation value of the canonical quark OAM can be expressed in terms of some TMDs [368-371]

$$
\begin{aligned}
\left\langle L_{\mathrm{can}}^{q}\right\rangle & =\int d x d^{2} k_{\perp}\left[h_{1}^{q}\left(x, \boldsymbol{k}_{\perp}^{2}\right)-g_{1 L}^{q}\left(x, \boldsymbol{k}_{\perp}^{2}\right)\right] \\
& =-\int d x d^{2} k_{\perp} \frac{\boldsymbol{k}_{\perp}^{2}}{2 M^{2}} h_{1 T}^{\perp q}\left(x, \boldsymbol{k}_{\perp}^{2}\right) .
\end{aligned}
$$

Unfortunately, it has also been shown that the validity of these relations cannot be extended to QCD [372-374]. Although not exact, they remain phenomenologically interesting as they provide at least some indication about the sign and the magnitude of the canonical quark OAM. Note that the results can be quite different $[375,376]$ from the kinetic quark OAM derived from the Generalised Parton Distributions (GPDs) through the Ji relation [14] which contains also quark-gluon interactions. 
Table 19

The Bethe-Heitler cross section and number of events predicted [389] for three different modes of operation for AFTER@LHC [Note that the assumed luminosity in [389] may not correspond to yearly luminosities].

\begin{tabular}{llll}
\hline & $\sigma_{B H}(\mathrm{pb})$ & Luminosity & Events year \\
\hline$p$ on $\mathrm{Pb}$ & $1940 \mathrm{pb}$ & $0.16 \mathrm{fb}^{-1}$ year $^{-1}$ & $3 \times 10^{5}$ \\
$p$ on $\mathrm{H}$ & $7.1 \mathrm{pb}$ & $20 \mathrm{fb}^{-1}$ year $^{-1}$ & $1.4 \times 10^{5}$ \\
$\mathrm{~Pb}$ on $\mathrm{H}$ & $5500 \mathrm{pb}$ & $11 \mathrm{nb}^{-1}$ year $^{-1}$ & $6 \times 10^{3}$ \\
\hline
\end{tabular}

Interestingly, Burkardt $[377,378]$ suggested that the quark Sivers TMD $f_{1 T}^{\perp q}\left(x, \boldsymbol{k}_{\perp}^{2}\right)$ and the quark GPD $E^{q}(x, \xi, t)$ could be related by a chromodynamic lensing mechanism

$$
\int d^{2} k_{\perp} \frac{\boldsymbol{k}_{\perp}^{2}}{2 M^{2}} f_{1 T}^{\perp q}\left(x, \boldsymbol{k}_{\perp}^{2}\right) \propto \int d^{2} b_{\perp} \overline{\mathcal{I}}\left(\boldsymbol{b}_{\perp}\right)\left(\boldsymbol{S}_{T} \times \partial_{b_{\perp}}\right)_{z} \mathcal{E}^{q}\left(x, \boldsymbol{b}_{\perp}^{2}\right),
$$

where $\overline{\mathcal{I}}\left(\boldsymbol{b}_{\perp}\right)$ is called the lensing function and $\mathcal{E}^{q}\left(x, \boldsymbol{b}_{\perp}^{2}\right)=\int \frac{d^{2} \Delta_{\perp}}{(2 \pi)^{2}} e^{-i \boldsymbol{b}_{\perp} \cdot \boldsymbol{\Delta}_{\perp}} E^{q}\left(x, 0,-\boldsymbol{\Delta}_{\perp}^{2}\right) \cdot \boldsymbol{S}_{T}$ is the transverse spin of the proton, while $\xi$ and $t=-\Delta_{\perp}^{2}$ are the longitudinal and transverse components of the momentum transfer. The Sivers function could then be used to constrain the GPD $E^{q}$ and hence the kinetic OAM via the Ji relation. Despite some support from model calculations [352,378-380], such a relation can hardly be put on a firmer theoretical ground. A variation of it has however been used by Bacchetta and Radici [381] to fit SIDIS data for the Sivers effect with the integral constrained by the anomalous magnetic moments, leading to a new estimate of the total angular momentum $\left\langle\left\langle_{\text {kin }}^{q}\right\rangle\right.$, in good agreement with most common GPD extractions [382-386]. A similar relation may a priori hold in the gluon sector, but has never been investigated so far.

In fact, one should not expect any direct quantitative relation between TMDs and OAM since the latter requires some information about the correlation between the position and the momentum of the parton, information which is integrated out at the TMD level. However, TMDs provide essential information about several angular-momentum correlations [387] and can be used to constrain, to some extent, the nucleon wave function, indirectly providing us with valuable information about its OAM content. Thus the AFTER@LHC programme, with its unique capabilities of measuring quark and gluon TMDs, and in particular Sivers functions, can shed light on the partonic OAM by giving us a handle to constrain it.

\subsubsection{Ultraperipheral collisions}

Ultraperipheral collisions (UPCs) provide a unique way to study photoproduction processes in hadron-hadron interactions [388], also in the fixed target experiments [389]. Such processes are conveniently described in the Equivalent Photon Approximation, where the relation between the hadron-hadron cross section, $(d) \sigma^{h_{A} h_{B}}$, and the (differential) cross section for a photo-hadron scattering $\left(h_{A}\right.$ or $\left.h_{B}\right),(d) \sigma^{\gamma h_{A B}}$, is naturally given by the following convolution in the photon momentum, $k_{\gamma}$, of the photon fluxes $d n / d k_{\gamma}$ from each hadron and the aforementioned photo-hadron cross section:

$$
d \sigma^{h_{A} h_{B}}=\int d k_{\gamma}\left[\frac{d n^{h_{A}}}{d k_{\gamma}} d \sigma^{\gamma h_{B}}\left(k_{\gamma}\right)+\frac{d n^{h_{B}}}{d k_{\gamma}} d \sigma^{\gamma h_{A}}\left(k_{\gamma}\right)\right] .
$$

The relevant parameters of such photon beams, for various projectiles and targets at AFTER@LHC, can be found in Table 1 of [389].

Exclusive photoproduction processes which can be studied in the UPCs allow one to probe the internal structure of hadrons in terms of GPDs [390,391], which through the Ji's sum rule are directly related to the total OAM carried by quarks and gluons. They also allow one to explore the tri-dimensional "tomography" of hadrons [392].

One of such exclusive processes, that has not yet been measured, is Timelike Compton Scattering (TCS) [393], contributing to exclusive lepton-pair photoproduction. Although the purely electromagnetic Bethe-Heitler (BH) amplitude gives much larger contributions than the TCS one, it is possible to study the interference term between TCS and BH processes, which may be projected out through the analysis of the angular distribution of the produced leptons. Such an interference then allows one to extract information on the GPDs. The ratio of these two contributions for the kinematics relevant to AFTER@LHC was found to be of the order of 10\% [389].

Table 19 gathers the $\mathrm{BH}$ cross section, the luminosity and the yearly expected lepton-pair yields in 3 operation modes ${ }^{37}$ in the kinematical region where the TCS-signal extraction is possible. With a magnitude of $10 \%$ for the interference term, the azimuthal modulation should be observable in the 3 cases.

We also note that the cross sections for $\eta_{c}$ production by photon-pair fusion has been derived in [389]. This process, in particular in this energy range, is sensitive to the method used to compute the photon flux in the $p p$ case. We found out that $10^{4} \eta_{c}$ can be produced per year in $p p$ UPCs with AFTER@LHC. The same paper also discussed possible

\footnotetext{
37 The Pb target should be considered as an illustrative case for a heavy nuclear target.
} 


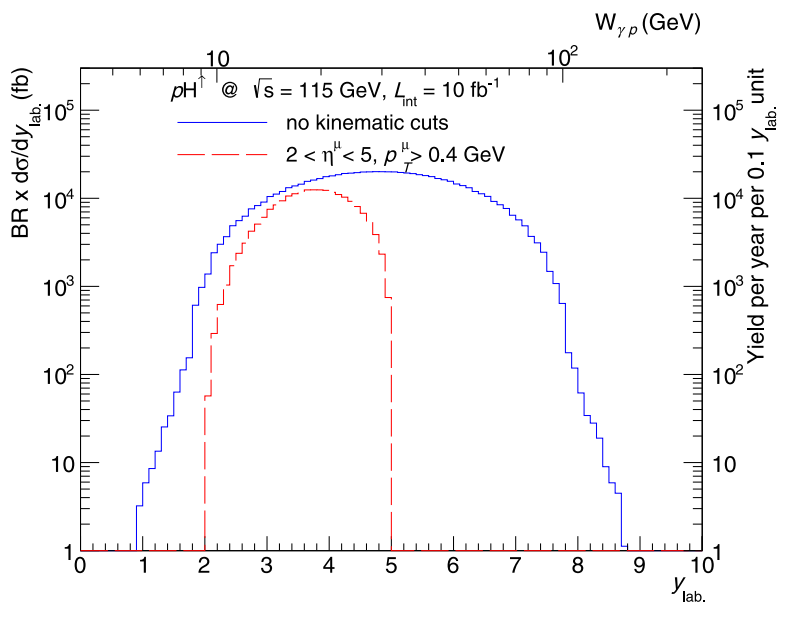

(a)

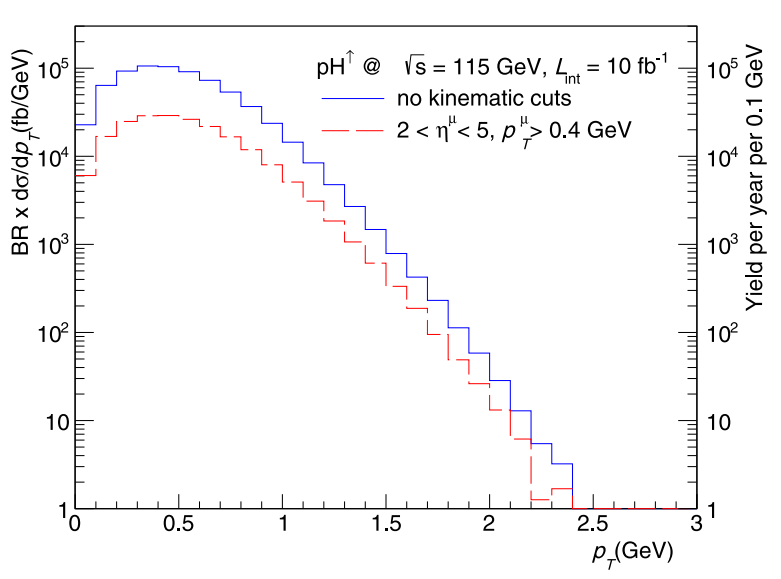

(b)

Fig. 40. Rapidity-differential (a) and $p_{T}$-differential (b) cross sections of the photoproduced $J / \psi$ in the laboratory frame for $p \mathrm{H}$ with $7 \mathrm{TeV}$ proton beam, from the STARLIGHT generator [395]. The blue curves have been produced without applying kinematical cut, while the red curves are produced by applying cuts on the two daughters of the $J / \psi\left(2<\eta^{\mu}<5\right.$ and $\left.p_{T}^{\mu}>0.4 \mathrm{GeV} / c\right)$ which correspond to a LHCb-like detector. For (a), the upper $x$ axis indicates the corresponding invariant mass of the initial $\gamma p$ system, $W_{\gamma p}$, which is equal to that of the final $J / \psi p$ system. Source: Adapted from [396].

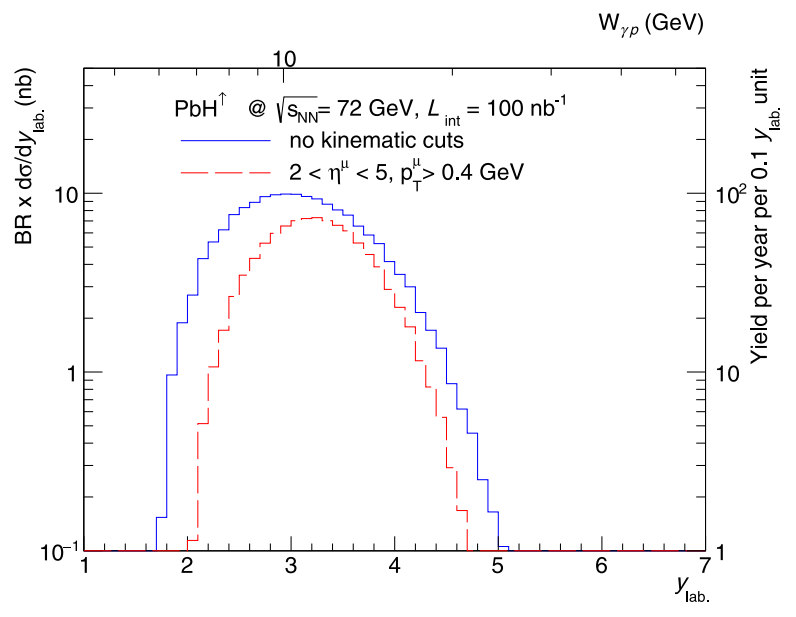

(a)

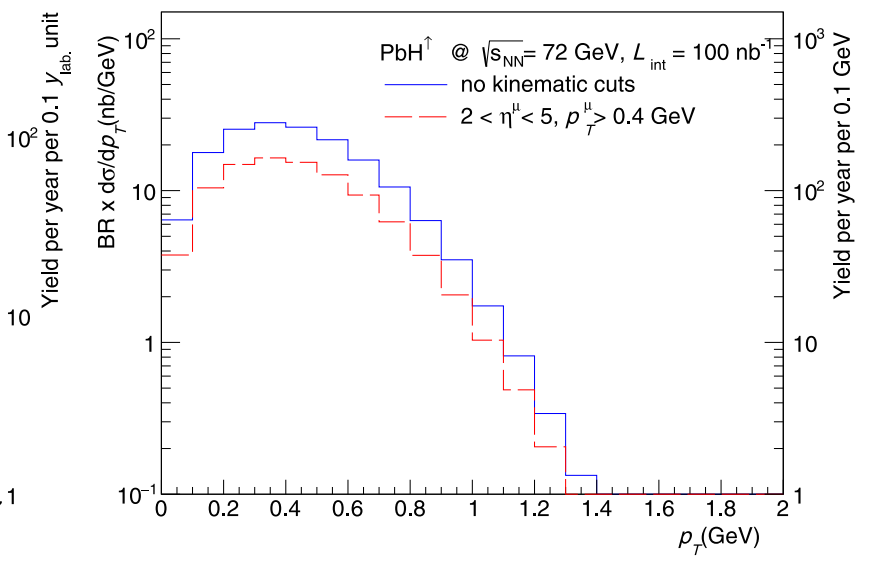

(b)

Fig. 41. Rapidity-differential (a) and $p_{T}$-differential (b) cross sections of the photoproduced $J / \psi$ in the laboratory frame for PbH with $2.76 \mathrm{~A}$ TeV $\mathrm{Pb}$ beam, from the STARLIGHT generator [395]. The blue curves have been produced without applying kinematical cut, while the red curves are produced by applying cuts on the two daughters of the $J / \psi\left(2<\eta^{\mu}<5\right.$ and $\left.p_{T}^{\mu}>0.4 \mathrm{GeV}\right)$ which correspond to a LHCb-like detector. Source: Adapted from [396].

competing hadronic processes via pomeron or odderon exchanges. These could be separated out by a careful analysis of the transverse-momentum dependence of the produced particles. $\eta_{c}$ production from $\gamma \gamma$ fusion in UPCs at AFTER@LHC was also discussed in [394].

Exclusive $J / \psi$ production [397] draws a lot of attention due to the fact that at the leading order it is only sensitive to gluon GPDs. It has already been measured in the ultraperipheral collisions at LHCb, ALICE and CMS in the collider mode. However, the AFTER@LHC programme would create a unique possibility to study STSAs in such a process [398], which are sensitive to the yet unknown GPD $E_{g}$ [399], an important piece of the spin sum rule. An analogue UPC process [400], where a $J / \psi$ is (semi-)inclusively photoproduced, would however give access to the gluon Sivers function.

In $[396,398]$ two LHC fixed-target operation modes were studied: proton-hydrogen and lead-hydrogen collisions. The $y$ distribution and $p_{T}$ distribution for those cases are shown in the Figs. 40 and 41. The statistical uncertainties in the bins relevant for the GPD extraction are presented in the Fig. 42, and indicate that AFTER@LHC is able to perform the 


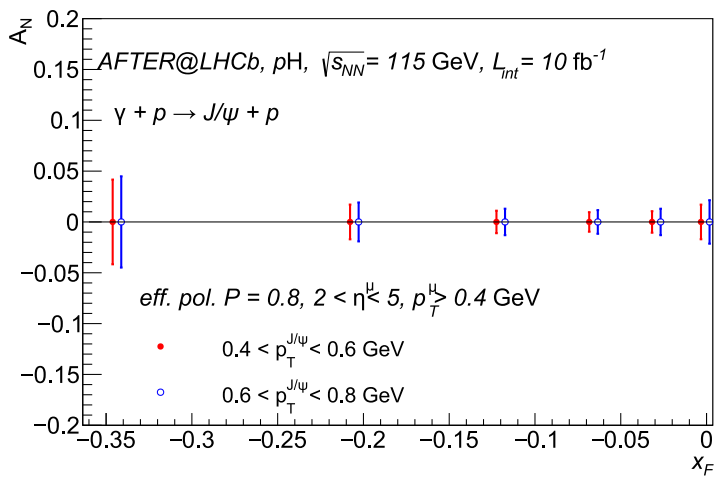

(a) $p \mathrm{H}$ with $7 \mathrm{TeV}$ proton

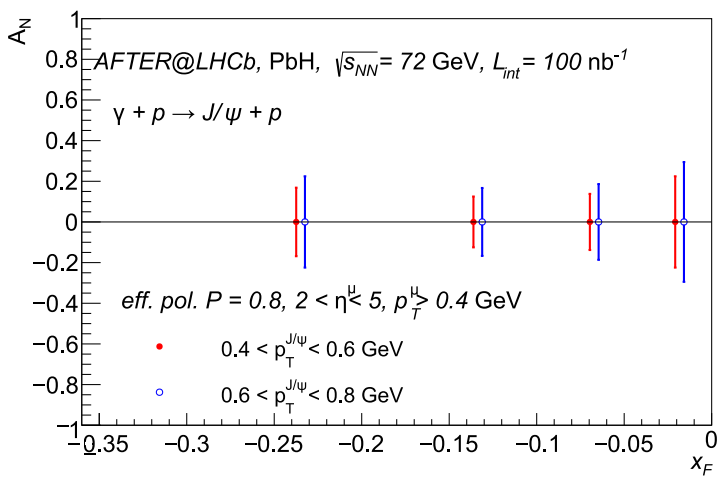

(b) $\mathrm{PbH}$ with $2.76 \mathrm{TeV} \mathrm{Pb}$

Fig. 42. Statistical uncertainty projections for the STSA in exclusive $J / \psi$ photoproduction in UPCS of a proton beam (a) and a lead beam (b) on a hydrogen target.

Source: Adapted from [396].

first determination of $E_{g}$. The exclusive vector-meson photoproduction in UPCs at AFTER@LHC was also studied in [401], including that of the light $\rho$ and $\omega$ mesons.

\subsubsection{Accessing the strange quark-helicity densities at high $x$}

The distributions of longitudinally polarised (anti-)quarks inside a nucleon are far from well known. For instance, there are still sizeable uncertainties in the case of anti-quarks (see e.g. [402]). Specifically, the understanding of the distribution of polarised strange and anti-strange quark distributions ( $\Delta s$ and $\Delta \bar{s}$, respectively), and their possible asymmetry is one of the most intriguing open quests in hadronic physics. Besides of being a key information on the structure of matter, it is also an essential ingredient of theoretical calculations in astrophysics ( $\Delta s$ enters the hydrodynamical modelling of core-collapse supernova explosions [403,404]). As of today, the precise value of $\Delta s$ remains unknown, although the experimental data of COMPASS [22] and several lattice calculations [405-409] are suggesting a small negative value while, at large $x$, HERMES data hint at a slightly positive value [410]. The region where the quarks carry the majority of the proton momentum (high $x, x \sim 1$ ) is of special importance. It is pivotal to reveal the content of nucleons observed in nature: both the quark structure and the total spin of a nucleon are in fact arising from the (valence) quark PDFs at high $x$. Currently, insufficient data exist in this kinematic range, which leads to unacceptably large uncertainties in the extracted polarised quark PDFs at $x \rightarrow 1$. For example, the $d / u$ quark ratio at high $x$ remains a puzzle (see Section 5.1.1). In general, any additional data at high $x$ can reduce the uncertainties in the determination of (un)polarised quark distributions. The AFTER@LHC programme provides opportunities for such studies. The ALICE CB detector used in the fixed-target mode covers extremely backward rapidities in the c.m.s., corresponding to $x \rightarrow 1$ in the target (see Section 4.2.1).

Moreover, the ALICE CB detector excels in particle identification and is capable of measuring identified hadrons (for example $\pi^{ \pm}, K^{ \pm}, K_{S}^{0}$ mesons and $\Lambda$ baryons). It can therefore provide data to study quark and anti-quark densities at $x \rightarrow 1$. Specifically, it gives access to the strange quark helicity densities at high $x$ via the measurement of the longitudinal spin transfer $D_{L L}$ from a longitudinal polarised target to $\Lambda$ and $\bar{\Lambda}$ hyperons. So far, only limited set of experimental $D_{L L}$ results exist $[411,412]$ and $[413,414]$ and their precision is far from being satisfactory.

The $\Lambda$ (and $\bar{\Lambda}$ ) baryons, which contain a strange (anti-strange) quark, are popular tools in studies of spin effects in high-energy collisions because of their self-spin analysing decays in $p \pi^{-}\left(\bar{p} \pi^{+}\right)$. In this decay, a proton is preferably emitted along the spin direction of a parent baryon, which gives a convenient access to the spin orientation of the latter. In practice, $D_{L L}$ is defined as the ratio of the difference of the inclusive cross sections with a positive or negative polarisation to their sum for a given target polarisation:

$$
D_{L L}^{\Lambda} \equiv \frac{\sigma_{p p \rightarrow \rightarrow \Lambda^{\prime}}-\sigma_{p p \rightarrow \rightarrow \Lambda^{\leftarrow}}}{\sigma_{p p \rightarrow \rightarrow \Lambda \rightarrow}+\sigma_{p p^{\rightarrow} \rightarrow \Lambda^{\leftarrow}}},
$$

where the $\rightarrow$ and $\leftarrow$ signs denote positive or negative helicities. Within perturbative QCD, $D_{L L}$ is sensitive to both polarised quark densities and polarised fragmentation functions. However, the interpretation of the experimental data depends on the assumed theoretical model of a fragmentation function. Besides the strange quark, the spin transfer from $u$ and $d$ quarks could contribute to $D_{L L}^{\Lambda}$ (see for example [415]); $D_{L L}^{\bar{\Lambda}}$ provides a cleaner probe of polarised anti-strange quark density. In what follows, we will omit the $\Lambda$ superscript of $D_{L L}^{\Lambda}$.

For the evaluation of the expected statistical precision of $D_{L L}$ measurements, we assumed that one will use a similar technique as in [411]. We estimated the statistical uncertainty on $D_{L L}$ taking $\sigma\left(D_{L L}\right)=\frac{1}{\alpha_{\Lambda} P} \frac{1}{\sqrt{N}}$, where $P$ is an effective target polarisation, $\alpha_{\Lambda}=0.750 \pm 0.010$ [416] is the weak decay parameter and $N$ is the overall $\Lambda$ yield registered in the ALICE detector. The $\Lambda$ production is simulated with the PYTHIA8 event generator, and our estimation takes into account the 


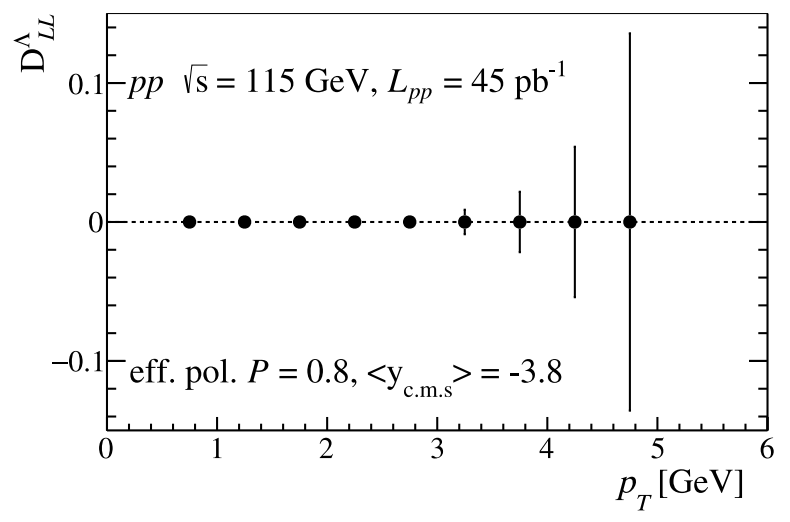

(a)

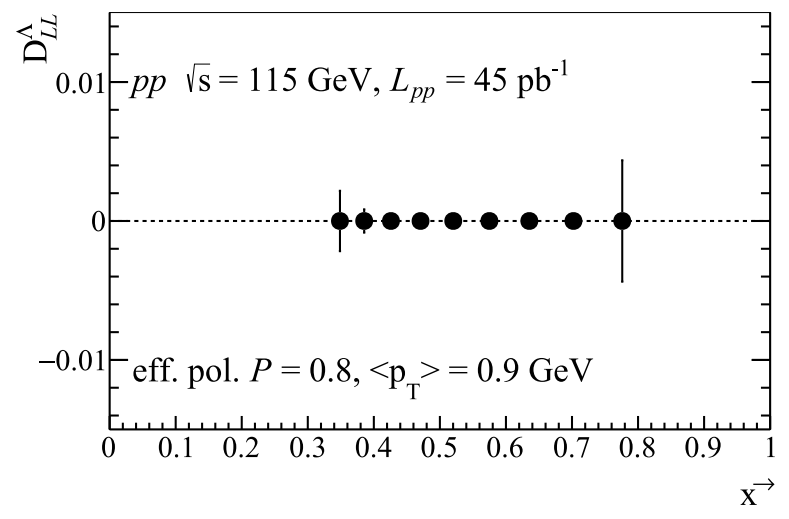

(b)

Fig. 43. The statistical precision expected for the longitudinal spin transfer $D_{L L}$ to $\Lambda$ hyperons with the ALICE detector and a target located ahead from the ALICE TPC at $z_{\text {target }}=-4.7 \mathrm{~m}$. The $\Lambda$ yields are calculated using PYTHIA, taking into account the acceptance of the ALICE TPC detector and a realistic reconstruction efficiency of $\Lambda$ baryons in the ALICE experiment [417].

geometrical acceptance of the ALICE CB detector for the $\Lambda$ daughters and the overall $\Lambda$ reconstruction efficiency [417]. Fig. 43 shows the expected statistical precision of $D_{L L}$ as a function of (a) the transverse momentum and (b) $x^{\rightarrow}$ for a target located in front of the ALICE TPC detector along the beam direction $\left(z_{\text {target }}=470 \mathrm{~cm}\right)$. The assumed integrated luminosity reflects the expected performance of the ALICE TPC detector and the target polarisation corresponds to a polarised hydrogen gas-jet target (see Section 3.3.2 for details).

With a single year of data taking it will be possible to measure $D_{L L}$ with sub-percent precision for $x \rightarrow \rightarrow$; however, the $p_{T}$ reach is limited due to the proximity of the end of the phase space. Therefore, these results could be a challenge for theoretical calculations, given the relevance of perturbative QCD calculations in these predictions. Anyhow, such data could give a unique opportunity to study polarised strange and anti-strange quark distributions at $x \rightarrow 1$.

As a final remark, we note that similar studies with a LHCb-like detector remain to be explored.

Strange-quark-transversity distribution. The integrated quark transversity, also called the nucleon tensor charge, is a useful input in the search for new physics beyond the standard model, especially in the context of the electric dipole moment [418,419] or the neutron beta decay [420]. The quark transversity of light quarks has recently been extracted through a TMD-evolution analysis of the Collins azimuthal asymmetries in $e^{+} e^{-}$annihilation and SIDIS processes [421], as well as through a global analysis of ep DIS and $p p$ collisions [422]. The tensor charges deduced from the above analyses are consistent with each other, but are in conflict with recent lattice QCD results [407,423-425]. The extraction of the nucleon tensor charge from experimental data is now under intense debate, and an improvement of the analysis of DIS experimental data considering hadrons in jet final states was recently proposed $[426,427]$.

Complementary measurements of the transverse-spin transfer $D_{T T}$ to hyperons can be carried out within the AFTER@LHC programme with a similar precision as that for $D_{L L}$, discussed above. $D_{T T}$ is sensitive to both the transversity distribution of $s$ and $\bar{s}$ and transversely-polarised fragmentation functions. Thus, $D_{T T}$ gives insight into transversity distribution of quarks which has never been extracted from experimental data (as well as the transversely-polarised fragmentation functions). However, the extraction of the nucleon tensor charge would require a reliable model of the fragmentation functions.

Recent lattice calculations suggest a small value of $\delta s \sim O\left(10^{-3}\right)$ for the strange quark contribution to the nucleon tensor charge $[425,428]$. Recently the STAR experiment reported $D_{T T}$ in polarised proton-proton collisions at $\sqrt{s}=200$ $\mathrm{GeV}$ at mid-rapidity (that is, in the low- $x$ regime), and indeed $D_{T T}$ is compatible with zero within uncertainties [429]. The sea-quark distributions (at large $x$ ) will hardly be measurable at any other experimental facility, and thus the AFTER@LHC programme might provide a very useful handle to further constrain the nucleon tensor charge and the transversely polarised fragmentation functions.

\subsection{Heavy-ion physics}

Despite considerable progress achieved in the last three decades at AGS, SPS, RHIC and LHC in understanding the properties of the hadronic matter at extreme conditions [95,97,430-432] produced in high-energy proton-nucleus and nucleus-nucleus collisions, crucial aspects of the resulting system remain obscure. Important open questions regarding the properties of a new phase of matter, assumed to be a Quark-Gluon Plasma (QGP), remain to be addressed:

1. the nature of the phase transition between the hadronic matter and the deconfined phase of quarks and gluons;

2. the transport properties of this medium, including its specific shear viscosity; 
3. the interaction of hard partons with this medium and their energy loss via collisional and radiative processes;

4. the flavour dependence of the energy loss in the hot medium;

5. the thermodynamic properties of this hot medium.

In the following text, we focus on two types of observables used in QGP studies: modification of hadron yields in heavy-ion (or proton-nucleus) collisions compared to proton-proton reactions, and anisotropy of the azimuthal-angle distribution of hadrons in the final state. We quantify the medium effects on hadron yields using the nuclear modification factor, $R_{A A}$ (or $R_{p A}$ ), which is a ratio of invariant yields in $A A(p A)$ interactions, normalised by the respective numbers of binary collisions, and yields in $p p$ collisions. If heavy-ion reactions are a simple superposition of $p p$ interactions, then $R_{A A} \sim 1$. The azimuthal anisotropy is conventionally quantified using a Fourier series of the particle azimuthal angle $\phi$ distribution with respect to the reaction plane $\Psi$ (a plane defined by the beam and a vector connecting the centres of colliding nuclei):

$$
\frac{d^{2} N}{d p_{T} d \phi} \propto \sum_{n=1}^{\infty} 2 v_{n}\left(p_{T}\right) \cos (n(\phi-\Psi))
$$

where $v_{1}$ is called the directed flow, $v_{2}$, the elliptic flow and $v_{3}$, the triangular flow, etc.. The anisotropy parameters $v_{n}$ provide information about the early dynamics of the created system and the collective behaviour of hadrons under study. They can shed more light on the equation of state and the thermodynamic properties, like the shear-viscosity $\eta$ to entropy-density $s^{38}$ ratio $\eta / s$, of the QGP. Since $v_{n}$ are measured using final-state particles, they can contain signals from the QGP phase, fluctuations from the initial conditions as well as some final-state effects such as correlations of the decay products from short-lived hadron decays or jet correlations. There are several different experimental approaches to determine the $v_{n}$ parameters, for example using two- or multi-particle correlations. In general, the latter are the most sensitive to the collective behaviour of these particles.

The AFTER@LHC heavy-ion programme will take place at the c.m.s. energy of $72 \mathrm{GeV}$ with a $2760 \mathrm{GeV} \mathrm{Pb}$ beam. With lighter species the c.m.s. energy is only slightly larger. ${ }^{39}$ The Beam Energy Scan (BES) programme at RHIC has shown that in AuAu collisions at $\sqrt{S_{N N}}=62 \mathrm{GeV}$ the produced hadrons have a large elliptic flow [433-435] and jet-quenching effects were observed [436]. These results suggest that quarks and gluons are probably deconfined in this energy range, which implies that AFTER@LHC will be capable of studying both the properties of this deconfined medium and the phase transition. The large kinematic coverage of the available detectors together with different colliding systems, and high luminosities, will allow AFTER@LHC to deliver data that can provide definitive answers to aforementioned questions. The purpose of this section is to demonstrate how AFTER@LHC will be able to address them.

\subsubsection{Precise quarkonium studies in new rapidity and energy domains}

Since more than thirty years, studies of the production of various quarkonium states in heavy-ion collisions have been performed in order to provide insights into the thermodynamic properties of a possible deconfined matter, via the observation of a sequential melting of quarkonia. However, global analyses including SPS, RHIC and LHC data clearly show that such endeavour is much more complex than initially thought $[94,95,437]$. Many facts support this viewpoint: the complexity of both charmonium and bottomonium feed-downs combined with the absence of measurements of direct yields; the competition with conventional nuclear effects (see Section 5.1.2 for a discussion of one of them, the modification of the PDF in a nucleus), the intrinsic complexity of modelling such a new state of matter and the fate of these bound states when they cross it and finally the smaller cross section of these hard probes compared to light flavoured hadrons.

Indeed, while quarkonium production in $A A$ collisions was predicted to be suppressed (relative to the $p p$ case) due to the (Debye-like) screening of the $Q \bar{Q}$ potential in the deconfined medium, where the coloured charges are mobile, it is now becoming clear that dynamical effects beyond such a static screening should be taken into account. These are due to the Landau damping following from the inelastic scatterings of the pair with the constituents of the deconfined medium, or to colour rotations of the colour-singlet $Q \bar{Q}$ pair leading to its dissociation. Conversely, these colour rotations may also lead to quarkonium regeneration. At high energies, the charmonium production can even be more complex with the possibility of the recombination of uncorrelated charm and anti-charm quarks produced in the same collisions.

The bottomonium case seems to provide an easier path to (partially) achieve the goal of using quarkonium sequential suppression as a thermometer. Indeed, the three states are observable ${ }^{40}$ in the di-muon channel and $b \bar{b}$ recombination is far less likely. This is even more true in the energy range of AFTER@LHC. This will be the object of the first quarkonium section.

In what concerns the charmonium family, in spite of significantly larger cross sections, the situation is much more intricate and unquestionably calls for measurements which have never been done. Merely improving the precision of

\footnotetext{
38 Not to be confused with the centre-of-mass energy $\sqrt{\mathrm{s}}$.

39 For $2890 \mathrm{GeV}$ Xe beam, $\sqrt{s_{N N}}=73.7 \mathrm{GeV}$.

40 Despite the aforementioned caveats related to the oversimplified picture of the thermometer based on the uniqueness of the Debye screening, it is in any case mandatory to measure more than 2 states to calibrate it and then to "measure" a temperature.
} 


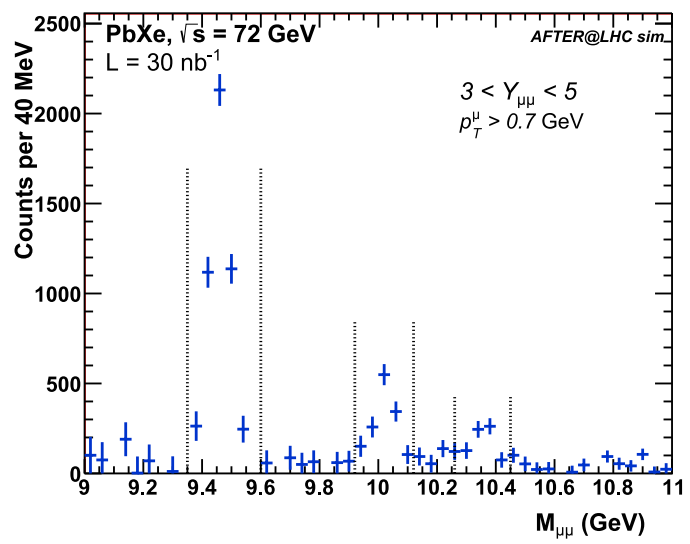

(a)

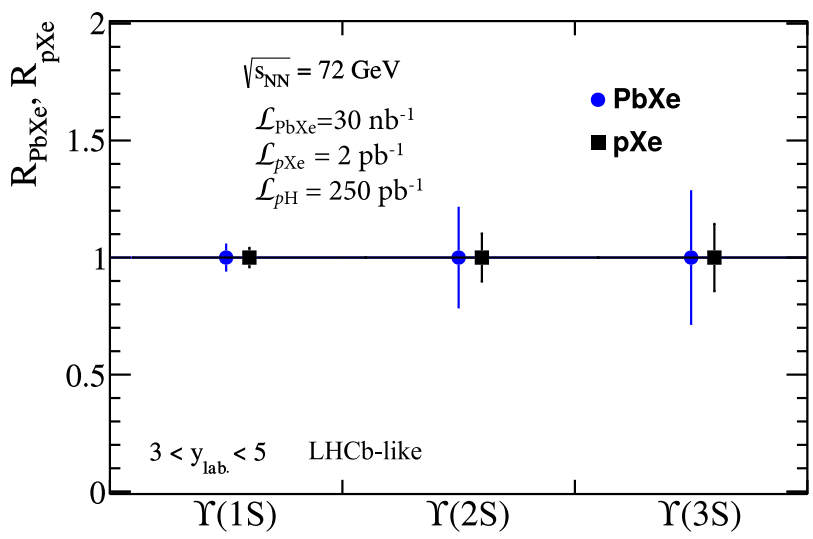

(b)

Fig. 44. (a) The $\Upsilon(n S)$ signal after the (like-sign) uncorrelated-background subtraction with the expected statistical uncertainties in PbXe collisions at $\sqrt{S_{N N}}=72 \mathrm{GeV}$ in the $\Upsilon$ acceptance range of $3<Y_{\mu \mu}^{\text {lab }}<5$, for a LHCb-like detector. No nuclear modifications are assumed, $\mathcal{L}_{\mathrm{PbXe}}=30 \mathrm{nb}^{-1}$. (b) Statistical-uncertainty projections for measurements of the nuclear modification factors $R_{\mathrm{PbXe}}$ and $R_{p X e}$ [441]. The uncertainties are calculated using the yields given in Table 20. [Adapted from [433].].

\section{Table 20}

$\Upsilon(n S)$ signal yields $(S)$ and signal-over-combinatorial-background ratios $(S / B)$ for $p p$, pXe and PbXe collisions at $\sqrt{S_{N N}}=72 \mathrm{GeV}$ and $3<y_{\mathrm{Lab}}<5$ assuming LHCb-like performances [441] with $\int \mathcal{L}_{p p}=250 \mathrm{pb}^{-1}, \int \mathcal{L}_{\mathrm{pXe}}=2 \mathrm{pb}^{-1}$ and $\int \mathcal{L}_{\mathrm{PbXe}}=30 \mathrm{nb}^{-1}$.

\begin{tabular}{lllllllll}
\hline$\Upsilon(1 S)$ & $S$ & $S / B$ & $\Upsilon(2 S)$ & $S$ & $S / B$ & $\Upsilon(3 S)$ & $S$ & $S / B$ \\
\hline$p p$ & $1.33 \times 10^{3}$ & 29.0 & $p p$ & $2.92 \times 10^{2}$ & 8.2 & $p p$ & $1.37 \times 10^{2}$ \\
$p \mathrm{Xe}$ & $1.39 \times 10^{3}$ & 7.8 & $p X e$ & $3.06 \times 10^{2}$ & 2.2 & $p$ Xe & $1.44 \times 10^{2}$ & 2.8 \\
$\mathrm{PbXe}$ & $4.33 \times 10^{3}$ & $1.8 \times 10^{-1}$ & PbXe & $9.56 \times 10^{2}$ & $5.0 \times 10^{-2}$ & $\mathrm{PbXe}$ & $4.49 \times 10^{2}$ & $6.2 \times 10^{-2}$ \\
\hline
\end{tabular}

past studies is bound to be insufficient. Indeed, the $\psi(2 S)$ state is likely too fragile to fit in any - idealised - thermometer picture. In addition, the access to information about a third state, in this case the $\chi_{c}$ triplet via feed-down measurements, has been shown to be close to impossible since there is no consensus, after 20 years of data, on whether they indicate that the $\chi_{c}$ suppression is closer to that of the $\psi(2 S)$ or of the $J / \psi$. In this context, AFTER@LHC can play a crucial role by providing completely novel observations ranging from direct- $\chi_{c}$-suppression measurements to new correlations studies. This will be the object of the second quarkonium section.

Measurements of the $3 \Upsilon(n S)$ states in $p p, p A$ and $A A$ collisions. In this context, we find it useful to start by discussing the unique reach of AFTER@LHC for $\Upsilon(n S)$ production in $p p, p A$ and $A A$ collisions as a function of the system size (for various colliding systems or vs. collision centrality), $p_{T}$ and rapidity, in $\mathrm{Pb} A$ collisions at $\sqrt{s_{N N}}=72 \mathrm{GeV}$. This lies nearly half way between the SPS and RHIC energy ranges and significantly lower than the only existing studies by CMS at the (collider mode) LHC [438-440]. Although the relative suppression of the $\Upsilon(n S)$ cannot readily be used as a thermometer, it will bring crucial new inputs for our understanding of the nature of the hot medium created in this energy range as opposed to that presently available at the LHC.

Fig. 44(a) shows the di-muon invariant-mass distribution in the $\Upsilon(n S)$ mass range for a single year of data taking with a LHCb-like set-up. The expected $\Upsilon(n S)$ yields are clearly large enough with the excellent resolution of LHCb to clearly distinguish each $\Upsilon(n S)$ state in an energy domain where $\Upsilon(n S)$ studies are extremely demanding. The yields together with the signal over background ratios in $p p, p X e$ and PbXe collisions are also gathered in Table 20. Projections of the statistical precision of the nuclear modification factors for the $\Upsilon(n S)$ states measured in $p$ Xe and PbXe collisions are presented in Fig. 44(b). The statistical uncertainties take into account the background-subtraction procedure using the like-sign method. If needed, this can further be improved by using the mixed-event technique. The predictions do not include potential modifications of the $\Upsilon(n S)$ yields due to the nuclear effects.

Fig. 45 shows the expected relative suppression $R_{\Upsilon(n S) / \Upsilon(1 S)} 41$ in the improved Comover Interaction Model (iCIM) recently applied to describe the $\Upsilon(n S)$ suppression at the LHC [442]. It can be seen as an effective approach to deal with quarkonium suppression, accounting for the Landau damping - the pair gets broken by a scattering with a gluon and the colour rotation - a scattering with a gluon turns the pair into a colour-octet state which cannot hadronise any more. Predictions in other approaches are expected to yield similar suppressions. Given the foreseen accuracy of $\Upsilon(n S)$ measurements, the AFTER@LHC programme will allow one to verify such predictions in a completely new energy domain.

41 The relative suppression $R$ is defined as the double ratio of excited $\Upsilon$ states to the $\Upsilon(1 S)$ in $A A$ and $p p$ collisions, $R \Upsilon(n S) / \Upsilon(1 S)=$ $[\Upsilon(n S) / \Upsilon(1 S)]_{A A} /[\Upsilon(n S) / \Upsilon(1 S)]_{p p}[438,443]$. 


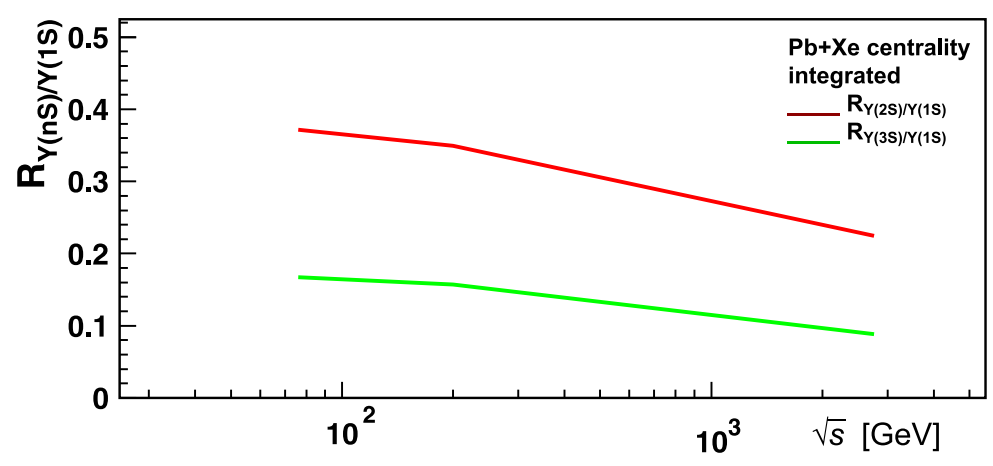

Fig. 45. The relative suppression of the excited $\Upsilon(n S)$ states to the $\Upsilon(1 S)$ as a function of the energy in iCIM [442].

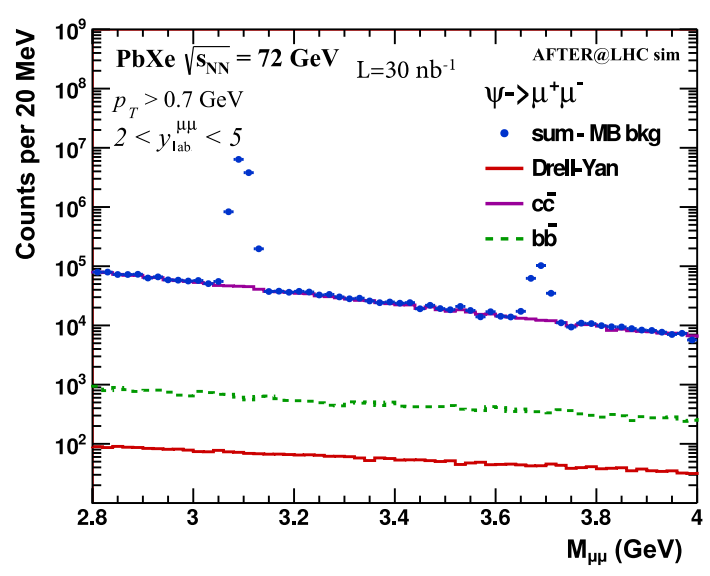

(a)

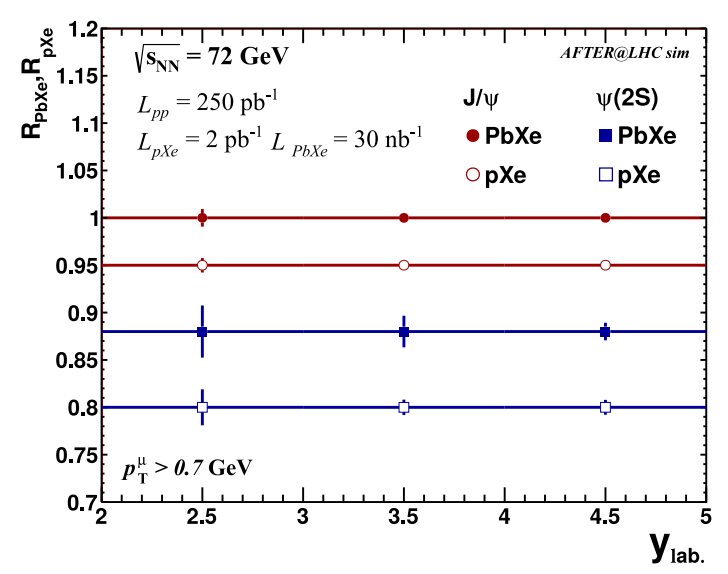

(b)

Fig. 46. (a) $J / \psi$ and $\psi(2 S)$ signal after the (like-sign) uncorrelated-background subtraction with the expected statistical uncertainties for PbXe collisions at $\sqrt{s_{N N}}=72 \mathrm{GeV}$ in $2<y_{\text {c.m.s. }}<5$ for $\int \mathcal{L}_{\mathrm{PbXe}}=30 \mathrm{nb}^{-1}$. No nuclear modifications are assumed. (b) Projected statistical precision of the nuclear modification factor as a function of the laboratory rapidity in PbXe $\left(R_{\mathrm{PbXe}}\right)$ and $p$ Xe $\left(R_{p X e}\right)$ at $\sqrt{s_{N N}}=72 \mathrm{GeV}$, assuming the uncorrelatedbackground subtraction with the like-sign technique using $\int \mathcal{L}_{p p}=250 \mathrm{pb}^{-1}, \int \mathcal{L}_{p X e}=2 \mathrm{pb}^{-1}, \int \mathcal{L}_{\mathrm{PbXe}}=30 \mathrm{nb}^{-1}$ which fits in one month of Pb ion run. Calculations were done for a LHCb-like detector performance.

Source: Adapted from [441].

Advanced charmonium studies in heavy-ion collisions. In addition to studies of the $\Upsilon(n S)$ states, the AFTER@LHC programme will explore an array of new charmonium observables that are virtually not accessible elsewhere. As compared to the SPS experiments, the higher energies at AFTER@LHC allow for quarkonium-correlation studies. None have been carried out so far in $p A$ and $A A$ collisions. The use of a detector like LHCb (or maybe the joint usage of the ALICE CB (to detect a photon and a muon) along with the muon arm (to detect the second muon) like in [444]) without any absorber enables $\chi_{c}$ studies at backward rapidities where the multiplicities are reduced. As compared to RHIC and LHC experiments, which have to cope with a large combinatorial background, studies of the $\eta_{c}$ suppression should be within reach in $p A$ collisions and, possibly, in the most backward part of the acceptance, in semi-central $A A$ collisions. These studies would rely on the natural access towards negative rapidities in the c.m.s., on large luminosities typical of the fixed-target mode and on more modern detectors as compared to those used in the 90's at the SPS and, to a lesser extent, to the ageing RHIC detectors.

The particularly large $p A$ rates (orders of magnitude larger than those reachable at the collider LHC and RHIC) with a wide rapidity coverage are also crucial to disentangle between the different sources of the charmonium suppression. In that regard, the interpretation of such studies, in an energy range where the charm recombination cannot be relevant [445], will also be much easier. This asset should not be underestimated.

Overall, one expects the measurements of $\psi(2 S), \chi_{c}, J / \psi+J / \psi$ and $J / \psi+D$ productions and correlations to be possible, each of them with enough precision to bring in constraints to the charmonium-suppression puzzle. As an illustration, Fig. 46(a) shows a di-muon invariant mass distribution for PbXe collisions at $\sqrt{s_{N N}}=72 \mathrm{GeV}$ in the $J / \psi$ and $\psi(2 S)$ mass ranges. It is clear that the background is well under control yielding a very precise determination of the charmonium rates. With such a background for the $J / \psi$, we find legitimate to highlight the possibility for the aforementioned more demanding studies. 


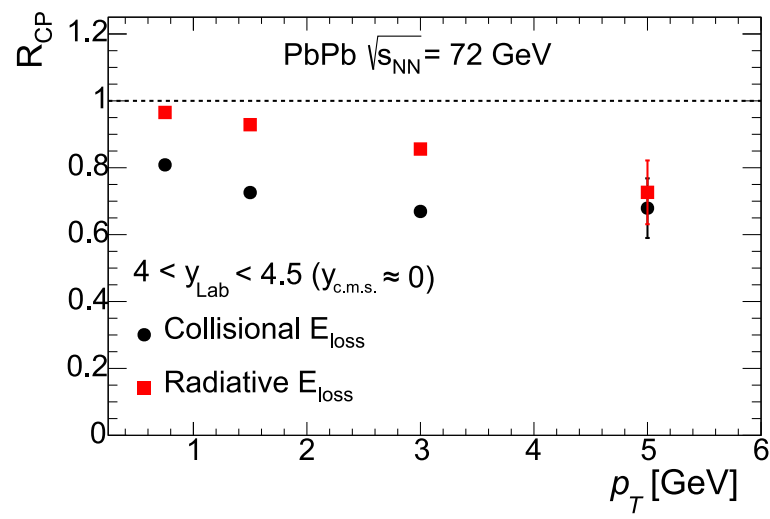

(a)

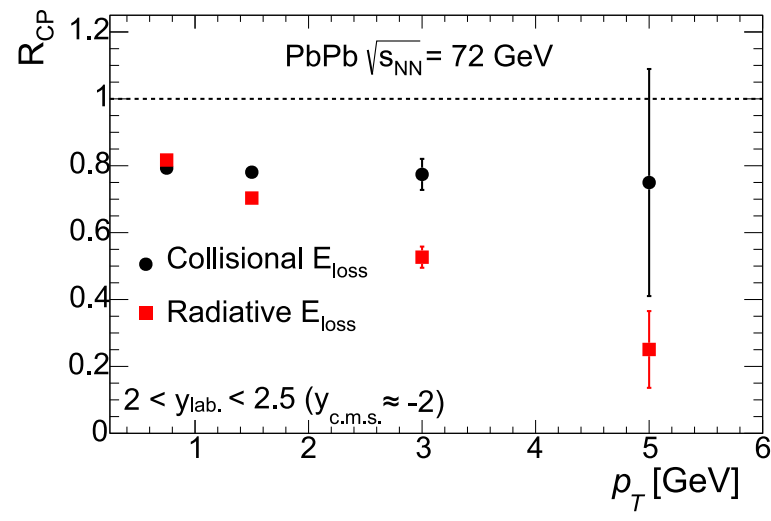

(b)

Fig. 47. Expected statistical uncertainties of $R_{C P}$ for $D^{0}$ mesons measured by a LHCb-like detector in PbPb collisions at $\sqrt{S_{N N}}=72 \mathrm{GeV}$ for $\int \mathcal{L}_{\mathrm{PbPb}}=$ $7 \mathrm{nb}^{-1}$.

Source: Adapted from [330].

Correspondingly, the statistical projections of the nuclear modification factors in $p X e$ and PbXe collisions as a function of the rapidity presented on Fig. 46(b) ${ }^{42}$ allows us to expect a precision at the per cent level for the $J / \psi$ and $\psi(2 S)$ cases, depending on the rapidity. Without a doubt, more differential studies as well as elliptic flow $v_{2}$ measurements will also be possible. Projections for the other charmonium-like observables remain to be done. However, we anticipate that they should show a precision around the five per cent level, which would be a clear breakthrough in the field since none of them are within the reach of any other experiment at present. Finally, let us stress that the present discussion only relied on a single target, Xe. However, almost independently of the target implementation (see Section 3), a few other species could be used - even during a single year. This will allow for systematic studies of the $A$ dependence of the nuclear effects to be complemented with that of the centrality.

\subsubsection{Study of the heavy-quark energy loss and their interaction with the surrounding nuclear matter}

Heavy quarks (charm and bottom) are unique tools to study and characterise the QGP properties. They are produced in hard scatterings at the early stage of the nuclear collisions and lose their energy while traversing the hot and dense medium. The energy loss and elliptic flow of open heavy-flavour hadrons are sensitive to the dynamics of the medium: such measurements could be used to determine the fundamental properties of the QGP, such as the transport coefficients, including $\hat{q}$ (which characterises the (squared) momentum transfer per mean free path of the fast partons) and the charm quark diffusion coefficients. Precision measurements of the elliptic flow of heavy quarks can give insights into the degree of thermalisation of the created nuclear matter (i.e. whether it is in a local thermal equilibrium or not) and can help discriminate between different models of the heavy-quark interactions with the QGP [95].

A significant suppression of open heavy-flavour production at high $p_{T}$ and a significant elliptic flow of the heavy quarks were observed at the top RHIC energy [446-449]. These experimental data can be described assuming two main effects: a medium-induced gluon radiation (radiative energy loss, $d E / d x_{\text {rad }}$ ) and a collisional energy loss, $d E / d x_{\text {coll }}$, due to binary interactions of the quark with other objects in the QGP. A major difficulty in modelling the heavy-quark energy loss is that the relative contributions of $d E / d x_{\text {coll }}$ and $d E / d x_{\text {rad }}$ are still not precisely known [95] and need to be constrained using experimental data. To better understand the interplay between both mechanisms, precise measurements of the individual suppression of charm and bottom quark yields are necessary. Fig. 47 shows calculations of the central-toperipheral nuclear modification factor $R_{C P}{ }^{43}$ for $D$ mesons as a function of $p_{T}$ in two rapidity ranges assuming $d E / d x_{\text {coll }}$ or $d E / d x_{\text {rad }}$ as the sole source of suppression. Since both mechanisms have a different $p_{T}$ and rapidity dependence, the high quality $D^{0}$ data to be provided by AFTER@LHC in PbA collisions at $\sqrt{s_{N N}}=72 \mathrm{GeV}$ in different rapidity ranges can help pin down $d E / d x_{\text {rad }}$ and $d E / d x_{\text {coll }}$.

With the high-statistics data accessible at AFTER@LHC, heavy-flavour azimuthal correlations $(D-D, J / \psi-D)$ and heavy-flavoured jets can also be studied to further understand the heavy-quark in-medium interactions. Simultaneous precise measurements of the $D$-meson elliptic flow and the nuclear modification factor will improve the determination of the QGP transport properties. In addition, correlation measurements of heavy-flavour pairs will provide new means to disentangle collisional from radiative interactions and to test Langevin against Boltzmann transport approaches [450]. In general, the temperature and mass dependences of the transport coefficients can be extracted with precise $R_{A A}$ and $v_{2}$

42 Statistical projections were calculated with the same assumptions as in the case of $\Upsilon(n S)$ predictions.

$43 R_{C P}$ is the ratio of the yields in central and peripheral collisions, normalised by the respective numbers of binary collisions. 
measurements for $B$ and $D$ at various beam energies. AFTER@LHC will clearly extend such studies towards the low-energy domain.

Furthermore, the study of $D$ mesons is a natural continuation of the investigation of the $J / \psi$ and $\psi(2 S)$ formation and dissociation in nucleus-nucleus collisions. Precise measurements of the $D$ yields in $p A$ and $A A$ collisions are necessary to constrain the initial-state cold nuclear matter effects, that is the modification of the $c \bar{c}$ production due to shadowing/antishadowing (see Section 5.1.2). In that sense, $D$ mesons are part of the useful measurements to establish a baseline to understand how the QGP affects quarkonium production. As we also discussed earlier, the nuclear effects on bottom-quark production can be also studied via $B \rightarrow J / \psi$ in $p A$ collisions with a good precision (see also [330]).

\subsubsection{Soft probes at large rapidities - a precise tool to study the bulk properties of the nuclear matter}

One of the unique assets of the AFTER@LHC programme stems from an expected large rapidity coverage. The combined acceptance of the LHCb detector and the central barrel of the ALICE experiment covers the range of $-5.2 \lesssim y^{\text {c.m.s. }} \lesssim 0.7$ in the case of the $2.76 \mathrm{TeV} \mathrm{Pb}$ beam - such a wide kinematic acceptance is not accessible in any collider experiment. Both detectors also offer excellent particle identification abilities, which altogether provide a large lever arm for studies related to the longitudinal expansion of the nuclear matter in heavy-ion collisions. In contrast, most of the heavy-ion experiments were designed to study the transverse dynamics of such a system at mid-rapidity, thus the longitudinal evolution is hardly accessible there.

The longitudinal dynamics of a system created in nuclear collisions is a topic of intensive experimental [451-453] and theoretical [454-457] studies via azimuthal flow, flow correlations and decorrelation measurements. The two- and multi-particle pseudo-rapidity correlations allow for an examination of the long-range collective phenomena, e.g. the ridge, as well as the initial-state fluctuations. The studies of the flow (de)correlation vs. rapidity offer an independent test of theoretical calculations that assume collective dynamics (collective flow). Most of them involve a hydrodynamic phase of the system evolution. Since the flow-decorrelation effect increases with the decreasing c.m.s. energy [458], AFTER@LHC will provide an excellent setting for such an analysis thanks to the aforementioned large rapidity coverage. Moreover, a measurement of the directed flow $v_{1}$ of charmed mesons as a function of rapidity was proposed recently to map out the three-dimensional distribution of the nuclear matter produced in heavy-ion collisions [459]. The large $D$ meson yields expected in AFTER@LHC will facilitate such a study with an unparallelled precision. In the following paragraphs, we discuss a few examples of other studies involving the "soft" (low $p_{T}$ ) probes that are pivotal for our understanding of the QGP properties and the QCD phase diagram.

Determination of the temperature dependence of the ratio of the shear viscosity to the entropy density: $\eta / s$. The shear viscosity $\eta$ (or the ratio of the viscosity to the entropy $s, \eta / s$ ), is one of the most fundamental properties of the QGP. Yet, our understanding of $\eta / s$ is far from satisfactory. Indeed, $\eta / s$ cannot directly be measured: it must be derived from a comparison of the experimental data and theoretical calculations (for example hydrodynamic models). After decades of developments, these models reached a high predictive power for the QGP macroscopic behaviour at RHIC and LHC energies. Until recently, such calculations focused on the transverse dynamics of the QGP at mid-rapidity. Intense efforts have been made to include the medium longitudinal expansion in hydrodynamic models (see e.g. [454]). These calculations indicate that particle yields and the azimuthal anisotropy coefficients $v_{n}$ measured at large rapidities are powerful tools to study the medium $\eta / s$ and its temperature dependence (see Fig. 6) [91].

The AFTER@LHC programme is well suited for these new frontiers of the QGP hydrodynamic studies, providing a large rapidity coverage to measure several particle azimuthal asymmetries and the possibility to obtain large statistics for different targets. Fig. 48(a)-(b) show particle yields as a function of pseudo-rapidity $\left(\eta_{\text {lab. }}\right)$ in mid-central (20\%-30\%) PbW and PbXe collisions at $\sqrt{s_{N N}}=72 \mathrm{GeV}$ obtained using EPOS. The expected statistical uncertainties on the elliptic flow ( $v_{2}$ ) measurement for identified hadrons with 10 million 20\%-30\% central PbW and PbXe events (i.e. 100 million minimum-bias events) are presented in Fig. 48(c)-(f). Even this small data sample, corresponding to an integrated luminosity of $14 \mu b^{-1}$ for PbW and $16 \mu \mathrm{b}^{-1}$ for PbXe collisions, will allow for a precision study of $v_{n}$ over a very broad rapidity range. Such high-quality data will allow for an accurate determination of the temperature dependence of $\eta / s$.

We believe that the AFTER@LHC programme is essential to advance our knowledge of the QGP macroscopic properties. This programme will complement and continue the existing studies of $v_{n}$ with high precision thus providing a detailed account of the system evolution (including transverse and longitudinal dynamics) between SPS and the top RHIC energy. As such, AFTER@LHC will be a perfect place to study collective effects in the system produced in $p A$ to PbA collisions. It will provide high precision studies of the QCD matter, complementary to the ones performed at the RHIC BES programme.

The rapidity scan: a new tool to study the QCD phase diagram. Thermal-model calculations indicate that the baryonic chemical potential $\mu_{B}$ and the temperature $\mathrm{T}$ depend on the rapidity [87]. The recent calculations using the Hadron Resonance Gas (HRG) model [88] and a viscous hydro+cascade model vHLLE+UrQMD [86] show that in PbPb collisions at AFTER@LHC $\mu_{B}$ strongly varies with the rapidity: $0<\mu_{B}<250 \mathrm{MeV}$ (as vHLLE+UrQMD predicts, see Fig. 5(b)) or even $80 \mathrm{MeV}<\mu_{B}<400 \mathrm{MeV}$ (given by the HRG model, Fig. 49(b)). These $\mu_{B}$ values cover a large fraction of the $\mu_{B}$ range accessible at the RHIC BES programme [90,460], as illustrated by Fig. 49(a). The $\mu_{B}$ vs. $y_{\text {c.m.s. }}$ dependence suggests that one can perform a "rapidity scan" of the QCD diagram [88,461], complementary to the BES programmes at RHIC and SPS. Measurements of correlations and fluctuations of the conserved quantities (electric charge, baryon and strangeness number, etc..) in small rapidity windows could provide a new approach to search for the QCD critical point and possible 


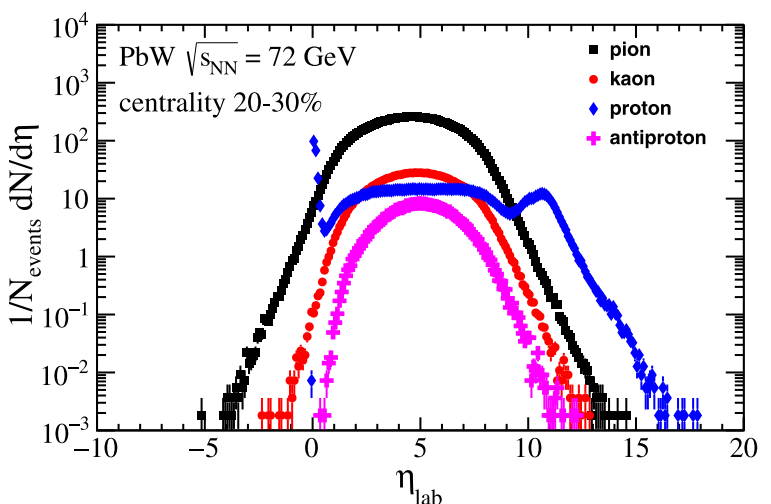

(a)

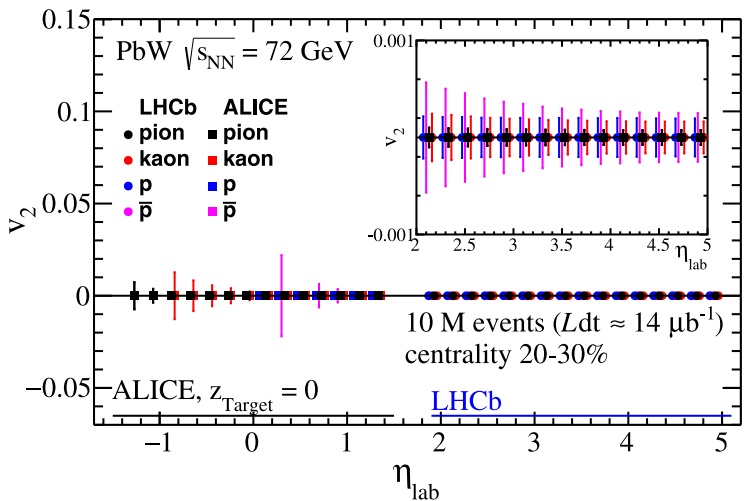

(c)

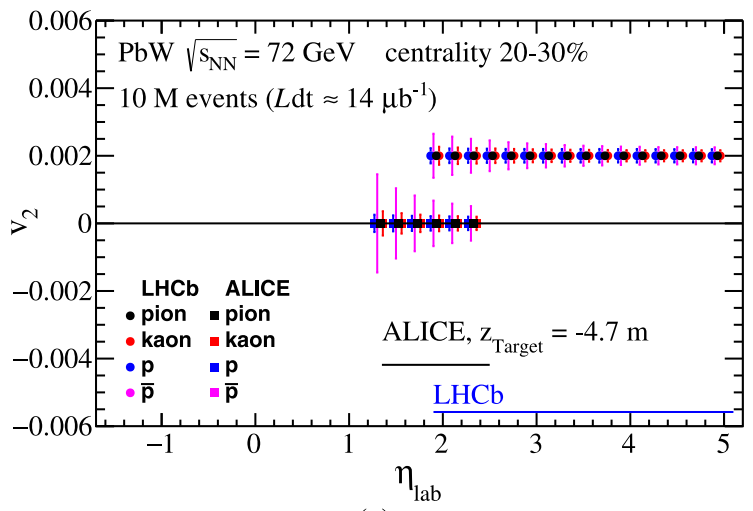

(e)

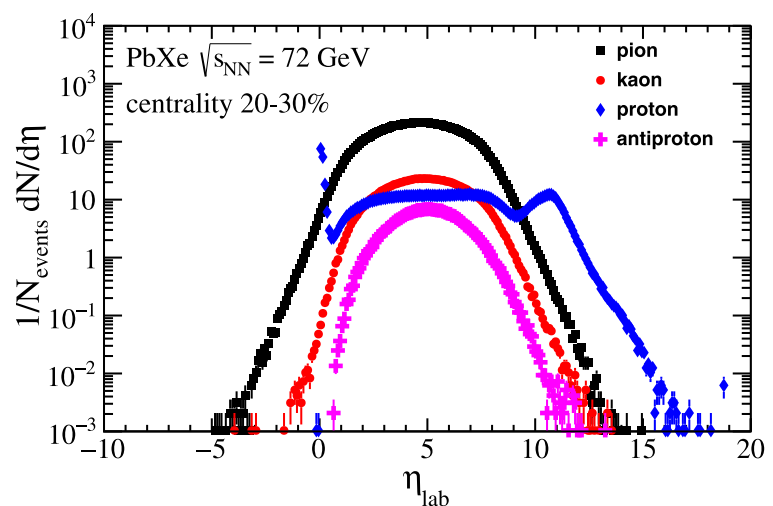

(b)

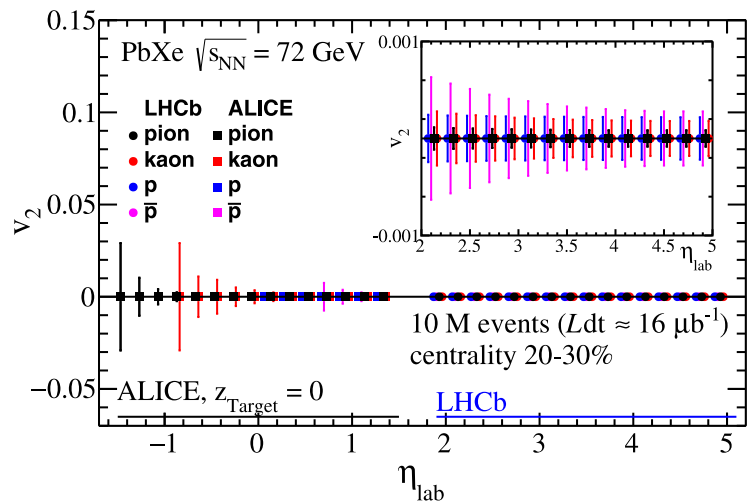

(d)

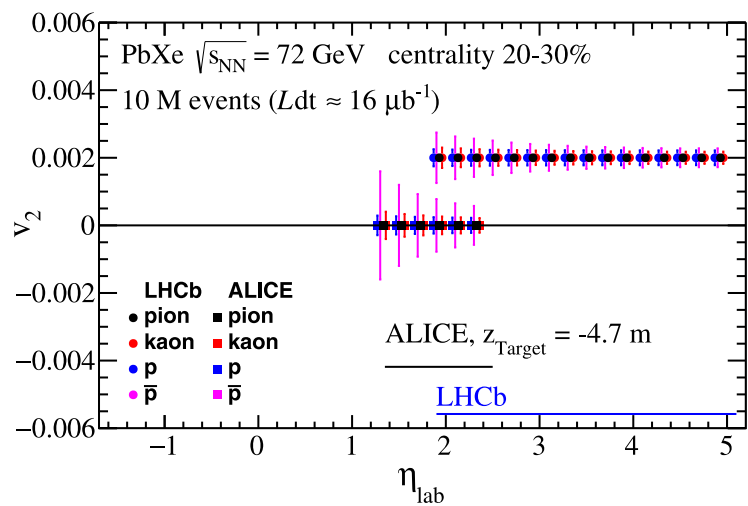

(f)

Fig. 48. Identified particle multiplicities per event in a simulation of (a) 5500 and (b) 9600 events of mid-central PbW and PbXe collisions at $\sqrt{s_{N N}}=72$ $\mathrm{GeV}$ and centrality 20\%-30\% from EPOS [114,159]. (c)-(f) Projections of the statistical uncertainty on the measurement of the $p_{T}$-integrated $\left(p_{T}>0.2\right.$ $\mathrm{GeV}$ for an ALICE-like set-up and $p_{T}>0.5 \mathrm{GeV}$ for a LHCb-like detector) elliptic flow of identified hadrons as a function of the pseudo-rapidity in the laboratory frame for two target locations for ALICE. The points for a LHCb-like detector in (e) and (f) were shifted vertically for clarity. Projections in (c) and (e) correspond to 10 million mid-central PbW collisions and the integrated luminosity $\mathcal{L}_{P b W}=14 \mu b^{-1}$, and the results in (d) and (f) represent 10 million mid-central PbXe events and $\mathcal{L}_{P b X e}=16 \mu \mathrm{b}^{-1}$.

evidences of the first-order phase transition. However, the interpretation of such a data could be a challenge since the origin of the $\mu_{B}$ vs. rapidity dependence is an open question. For example, it might result from a superposition of different rapidity spectra of particles produced in a single, homogeneous fireball; or a product of small subdomains with different $\mu_{B}$ and $\mathrm{T}$ values, moving with different longitudinal velocities. In the latter case, one could indeed perform phase-diagram studies by varying the particle rapidity. Nevertheless, the large rapidity coverage of the AFTER@LHC project, combined 


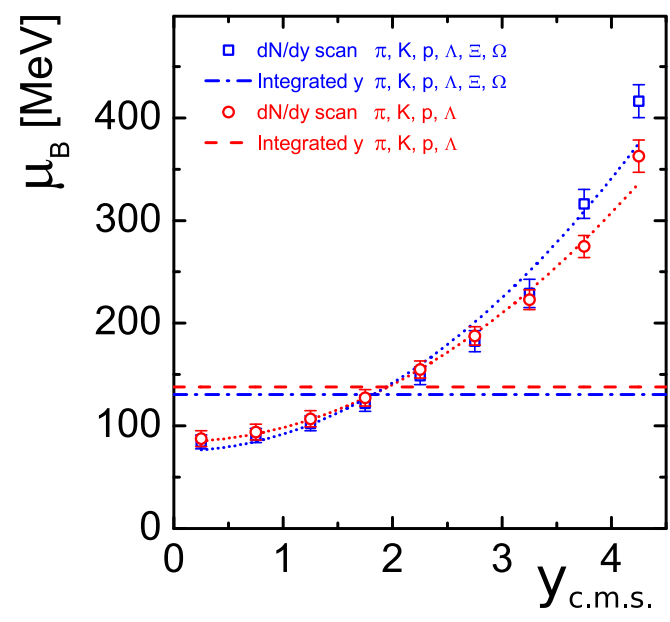

(a)

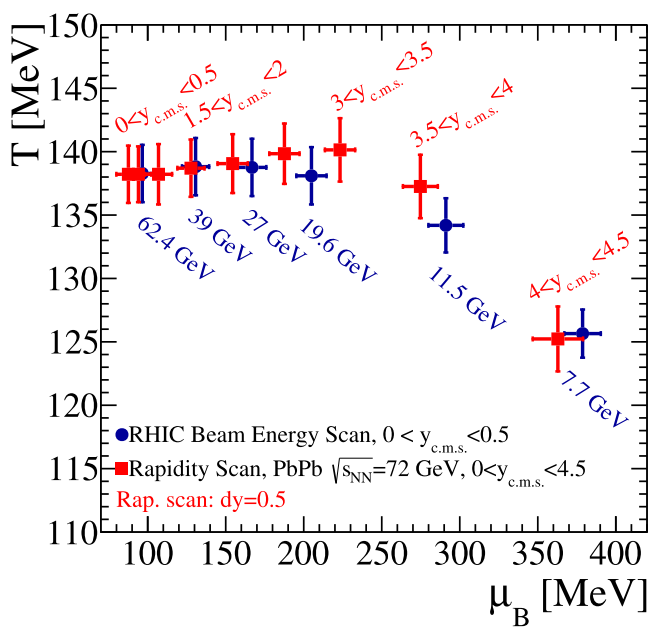

(b)

Fig. 49. (a) The baryonic chemical potential $\mu_{B}$ as a function of $y_{\text {c.m.s. }}$ and (b) the temperature $\mathrm{T}$ as a function of $\mu_{B}$ in $0 \%-10 \%$ most central $\mathrm{PbPb}$ collisions at $\sqrt{s_{N N}}=72 \mathrm{GeV}$ from the Hadron Resonance Gas model calculations [Adapted from [88]]. The two series of results represent calculations that use two sets of particle densities as the input (with or without the $\Xi$ and $\Omega$ baryons). The uncertainties on the points arise from an assumed relative uncertainty of $10 \%$ on the particle yields measured in AFTER@LHC.

with an excellent particle identification capabilities of the ALICE and the LHCb detectors, makes it a perfect place for such a "rapidity scan" of the QCD phase diagram.

\subsubsection{Search for a collective dynamics of partons in small systems at low energies}

One of the recently most discussed topics in the heavy-ion community is the possibility of collective motion of partons and formation of QGP droplets in so-called small systems such as $d A u, p A$ and even in $p p$ collisions. These considerations were triggered by an observation of long-range angular correlations of hadrons in high-multiplicity $p p$ events at the LHC [462], and later in $\mathrm{pPb}$ collision at the LHC [463] and dAu at RHIC [464]. At first, it was a surprise since such correlations were earlier observed only in heavy-ion collisions. Another unexpected result was a large asymmetry of azimuthal angle distributions of hadrons produced in high multiplicity $d A u$ [464] and $p \mathrm{~Pb}$ collisions [465]. A large, positive $v_{2}$ seen in the heavy-ion collisions is usually interpreted as an indication of collective interactions on the partonic level, thus of a possible QGP formation. Surprisingly, the values of $v_{n}$ observed in small systems are comparable to the ones observed in AuAu and $\mathrm{PbPb}$ collisions for the similar multiplicity events. Positive flow parameters were registered in essentially all kinds of hadronic collisions with energies as low as $\sqrt{s_{N N}}=19.6 \mathrm{GeV}$ [466]. Moreover, there is a significant positive elliptic flow of heavy-flavour particles and strange baryons in the $p A$ collisions at the LHC [467,468], although its magnitude is lower than the $v_{2}$ of light hadrons. There are also hints of non-zero $v_{2}$ of heavy quarks in dAu collisions at $\sqrt{s_{N N}}=200 \mathrm{GeV}$ at RHIC.

In addition, other intriguing phenomena were observed in high-multiplicity $p p$ interactions. The production of multistrange hadrons in such $p p$ collisions is enhanced [469], on a level remarkably similar to the results seen in PbPb collisions. Moreover, heavy-flavour-particle yields (both the open-heavy flavour and charmonium) increase fast with the number of charged particles produced in a pp collision [470-472]. A few different models are able to qualitatively reproduce such a behaviour, for example, the string percolation approach [473] or EPOS [474]. While these approaches significantly differ, they all assume some sort of collective interactions on the parton level.

Several explanations for the collective behaviour in small systems were suggested: formation of QGP droplets [475-478], parton escape with kinetic transport [479], coherent effects driven by a colour glass condensate [480], as well as more basic QCD derivations of string formation [481]. AFTER@LHC is in the optimal position to address this issue and discriminate between the proposed scenarios. The luminosity available with AFTER@LHC is orders of magnitude larger than that at RHIC. The differential measurement of $D$ and $J / \psi$ production and azimuthal asymmetries $v_{n}$ will be possible in $p p$ and $p A$ collisions. Fig. 50 shows the charged-particle-density distribution in $p p$ collisions at $115 \mathrm{GeV}$ from EPOS. AFTER@LHC will be able to probe very rare events, with a multiplicity of the order of 15 times larger than the average, and larger. The precision studies as a function of rapidity, transverse momentum and event multiplicity will shed new light on the problem at hand. As an example, Fig. 51 shows a statistical precision of the measurement of the elliptic flow $v_{2}$ of $D^{0}$ in $p \mathrm{~Pb}$ collisions with AFTER@LHC in two rapidity ranges - the statistical uncertainty is at a sub-percent level for $p_{T}<3 \mathrm{GeV}$. While very limited number of model predictions exist for charmed hadron $v_{2}$ in small-system collisions (for example the POWLANG [482] and Colour-Glass-Condensate (CGC) predictions [483] for $p$ Pb collisions at $\sqrt{s_{N N}}=8 \mathrm{TeV}$ ), one may consult relative variation of predictions for $v_{2}$ of $D^{0}$ in heavy-ion collisions at RHIC [446] to assess the discrimination 


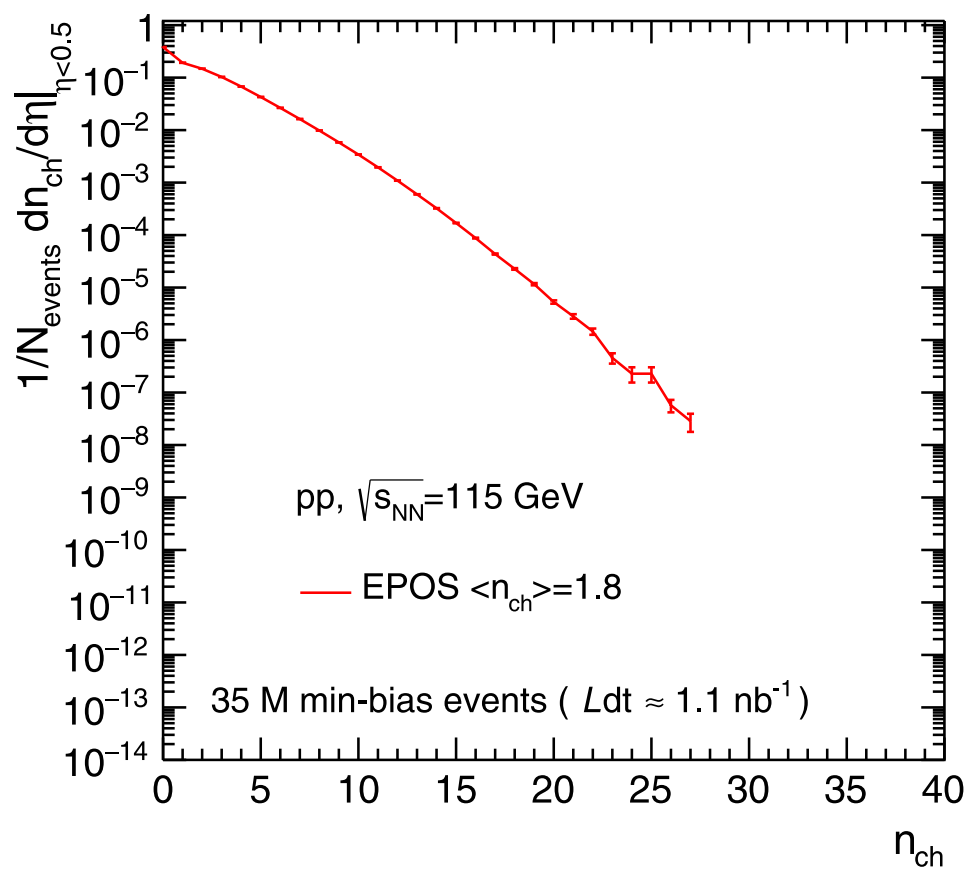

Fig. 50. Distribution of the charged-particle density in $p p$ collisions at $\sqrt{s}=115 \mathrm{GeV}$ at mid-rapidity from the EPOS model for 35 million minimum-bias events, which corresponds to an integrated luminosity of $1.1 \mathrm{nb}^{-1}$.

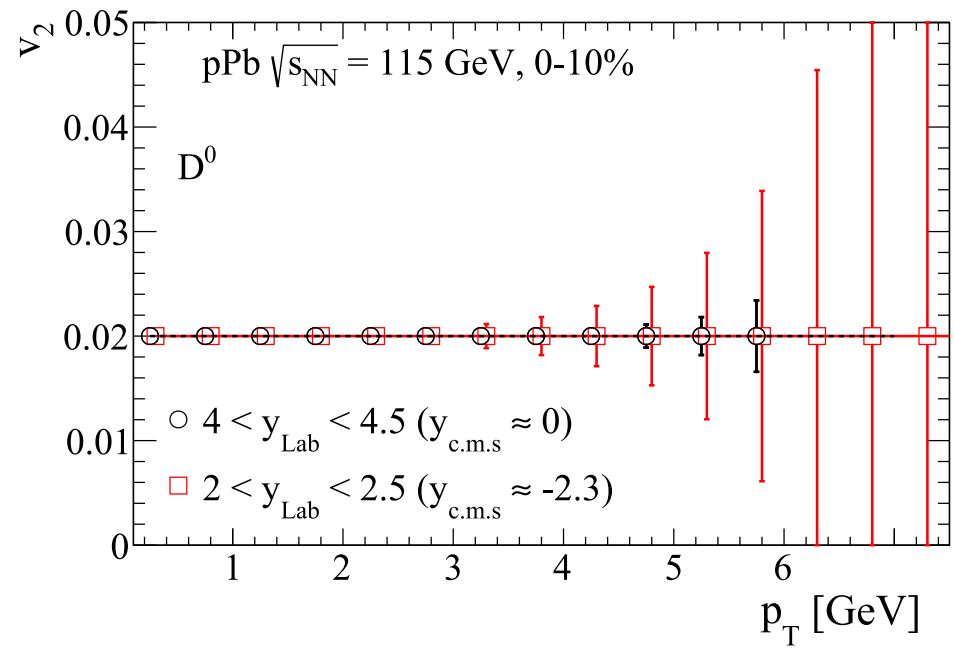

Fig. 51. Expected statistical uncertainties of $D^{0}$ elliptic flow in $p \mathrm{~Pb}$ collisions at $\sqrt{s_{N N}}=115 \mathrm{GeV}$ at mid-rapidity and backward rapidity measured with a LHCb-like detector for $\int \mathcal{L}_{p \mathrm{~Pb}}=160 \mathrm{pb}^{-1}$. Calculations include the acceptance and reconstruction efficiency of a LHCb-like detector. The results indicate that $v_{2}$ will be measured with sub-percent precision over a broad $p_{T}$ and rapidity range.

Source: Adapted from [330].

power of the proposed measurement at AFTER@LHC. At transverse momentum $p_{T} \approx 2 \mathrm{GeV}$ the difference between theory results is significantly larger than the estimated statistical uncertainty on $D^{0} v_{2}$ in Fig. 51 . Thus, together with the nuclear modification factor $R_{p \mathrm{pb}}$, these data will provide means to precisely determine a possible collective behaviour of heavy quarks and quantify the cold-nuclear-matter effects. Moreover, high-luminosity data samples available at AFTER@LHC will allow $v_{n}$ to be measured for identified hadrons with multi-particle-correlation methods over a wide rapidity range. This gives a useful handle on the collective effects in small systems. Consequently, AFTER@LHC offers novel opportunities to study these effects at low energies with multiple probes and over a broad kinematic range. 


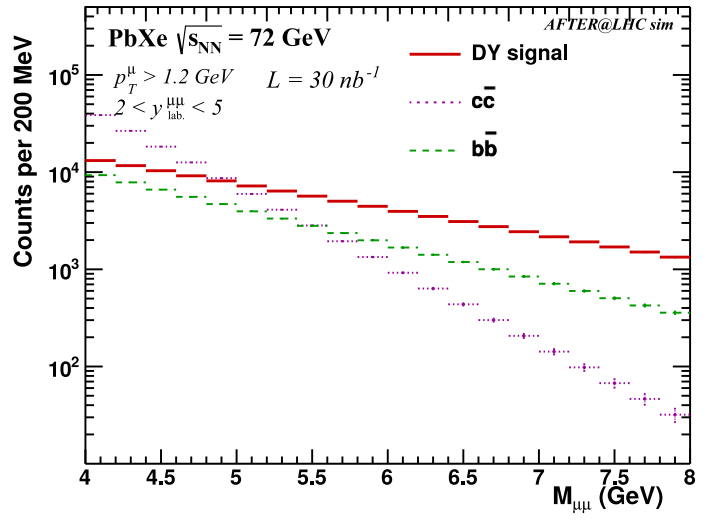

(a)

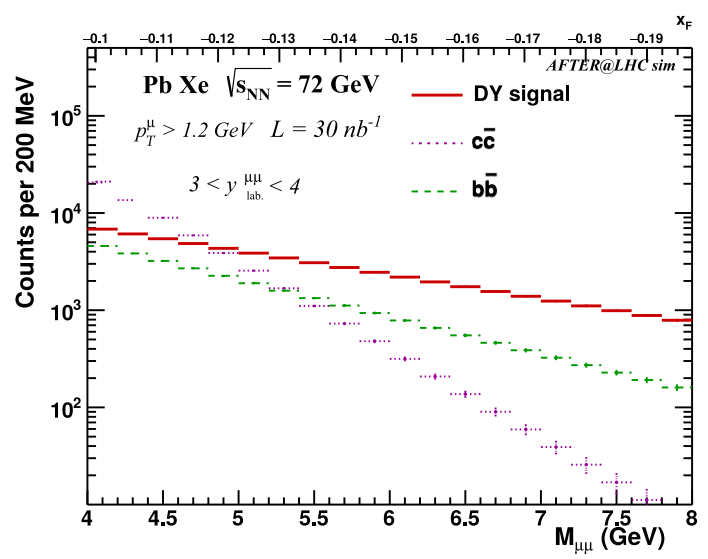

(c)

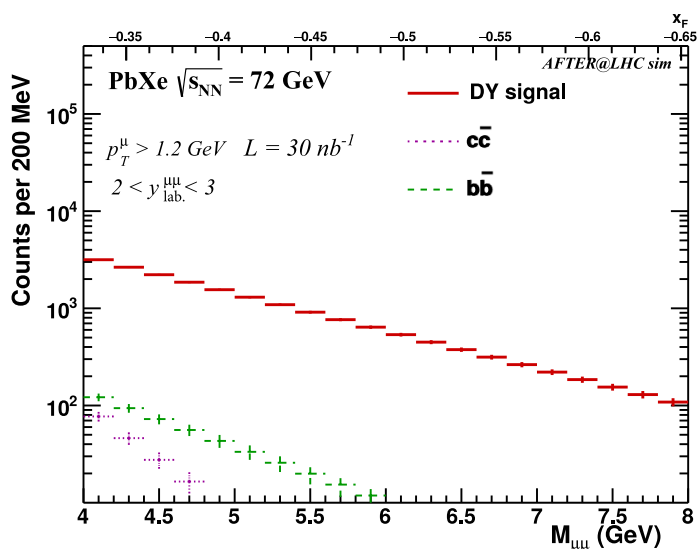

(b)

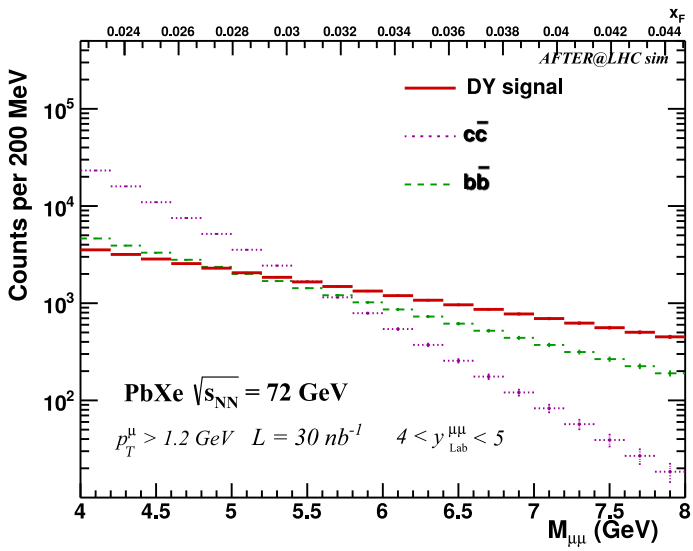

(d)

Fig. 52. Di-muon invariant-mass distributions $\left(4<M_{\mu^{+} \mu^{-}}<8 \mathrm{GeV}\right)$ from DY, $c \bar{c}$ and $b \bar{b}$ productions registered with a LHCb-like detector, for $P b X e$ collisions at $\sqrt{S_{N N}}=72 \mathrm{GeV}$ with $\int \mathcal{L}_{\mathrm{PbXe}}=30 \mathrm{nb}^{-1}$, assuming $R_{A A}=1$, in the integrated rapidity range of $2<y^{\mu \mu}$ lab. $<5$ (a) and divided into the following ranges: $2<y_{\text {lab }}^{\mu \mu}<3$ (b), $3<y_{\text {lab. }}^{\mu \mu}<4$ (c) and $4<y_{\text {lab }}^{\mu \mu}<5$ (d). For (b-d), the upper $x$-axis represents the corresponding $x_{F}$ values in a given rapidity range and invariant-mass bin. The combinatorial background is not presented and systematic uncertainties resulting from the background subtraction with the event-mixing technique are not included. [Adapted from [433].].

\subsubsection{Test of the factorisation of the initial-state nuclear effects in AA collisions with Drell-Yan lepton-pair production}

Initial-state effects observed in $p A$ collisions are currently extrapolated to $A A$ collisions assuming that the effects factorise linearly, that is, that the effects associated to initial-state sources are independent in each nucleon-nucleon binary collision. This naïve assumption can be tested using electromagnetic probes: high- $p_{T}$ isolated photons (inverse Compton process), DY, $W$ and $Z$ bosons. These probes are produced from initial-state partons and do not interact with the nuclear medium. The nuclear modifications observed for isolated photons, $W$ and $Z$ bosons measured by CMS at mid-rapidity [484-486] are smaller than $\sim 20 \%$ in $\mathrm{PbPb}$ collisions. These results rule out a scenario of a large suppression due to initial-state effects in heavy-ion collisions. However, they cannot test whether processes observed in $p A$ collisions are magnified in $A A$ collisions given the small nuclear modifications observed in $p A$ collisions to start with. Theoretical work such as that performed in [487] indicates that initial-state effects can be modified in $A A$ collisions with respect to $p A$ since the natural scale of a given process is boosted and thus affected by initial-state effects with a different magnitude. A conclusive experimental test of such a phenomenon from $p A$ to $A A$ collisions needs to be performed in a broad $x$ range where significant variation of the nuclear modifications is expected in $p A$ collisions (see the $p A$ physics section, Section 5.1.2).

The physics programme of AFTER@LHC includes the precise measurements of the DY process which can probe initialstate effects on quarks in several $A A$ collision species. The greatest challenge in the DY measurements in colliders is the large correlated background from $b+\bar{b} \rightarrow B^{+}+B^{-} \rightarrow l^{+} l^{-}$and $c+\bar{c} \rightarrow D^{+}+D^{-} \rightarrow l^{+} l^{-}$. This background is much smaller at the AFTER@LHC c.m.s. energy in $\mathrm{PbA}$ collisions. The large combinatorial background typically expected 


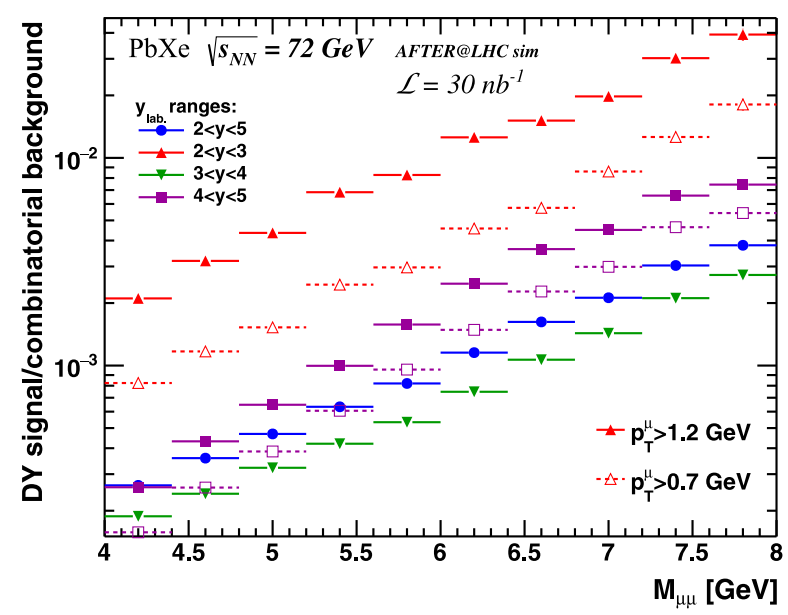

Fig. 53. DY signal-over-combinatorial-background ratio $(S / B)$ for a LHCb-like detector as a function of the di-muon invariant mass, for PbXe collisions at $\sqrt{s_{N N}}=72 \mathrm{GeV}$ with $\int \mathcal{L}_{\mathrm{PbXe}}=30 \mathrm{nb}^{-1}$ and for $2<y_{\text {lab. }}<5,2<y_{\text {lab. }}<3,3<y_{\text {lab. }}<4$ and $4<y_{\text {lab. }}<5$. The solid lines represent the $S / B$ with the selection condition for DY single-muon $p_{T}^{\mu}>1.2 \mathrm{GeV}$, and dashed lines represent the $S / B$ with $p_{T}^{\mu}>0.7 \mathrm{GeV}$. No nuclear modifications assumed. [Adapted from [441].].

Table 21

DY yields $\left(\times 10^{3}\right)$, uncorrelated background yields $\left(\times 10^{6}\right)$ and DY over the uncorrelated background ratios $\left(\times 10^{-3}\right)$ for PbXe collisions at $\sqrt{S_{N N}}=72 \mathrm{GeV}$ in 4 di-muon invariant-mass ranges between 4 and $8 \mathrm{GeV}$ and three rapidity ranges between 2 and 5, with single $\mu$ satisfying $p_{T}>1.2 \mathrm{GeV}$. The results hold for LHCb-like performances and $\mathcal{L}_{\mathrm{PbXe}}=30$ $\mathrm{nb}^{-1}$.

\begin{tabular}{|c|c|c|c|c|c|c|}
\hline & \multicolumn{3}{|l|}{$M_{\mu \mu}: 4-5 \mathrm{GeV}$} & \multicolumn{3}{|c|}{$M_{\mu \mu}: 5-6 \mathrm{GeV}$} \\
\hline & signal $\left(\times 10^{3}\right)$ & bkg. $\left(\times 10^{6}\right)$ & S/B $\left(\times 10^{-3}\right)$ & signal & bkg. & $\mathrm{S} / \mathrm{B}$ \\
\hline$y_{\text {lab. }}: 2-3$ & 11.48 & 4.38 & 2.6 & 4.77 & 0.76 & 6.3 \\
\hline$y_{\text {lab. }}: 3-4$ & 26.85 & 121.90 & 0.2 & 15.58 & 36.6 & 0.4 \\
\hline \multirow[t]{3}{*}{$y_{\text {lab. }}: 4-5$} & 15.33 & 45.08 & 0.3 & 8.28 & 8.05 & 1.0 \\
\hline & \multicolumn{3}{|l|}{$M_{\mu \mu}: 6-7 \mathrm{GeV}$} & \multicolumn{3}{|c|}{$M_{\mu \mu}: 7-8 \mathrm{GeV}$} \\
\hline & signal & bkg. & $\mathrm{S} / \mathrm{B}$ & signal & bkg. & S/B \\
\hline$y_{\text {lab. }}: 2-3$ & 1.92 & 0.14 & 13.9 & 0.82 & 0.03 & 28.1 \\
\hline$y_{\text {lab. }}: 3-4$ & 8.80 & 9.68 & 0.9 & 5.11 & 2.42 & 2.1 \\
\hline$y_{\text {lab. }}: 4-5$ & 5.09 & 1.68 & 3.0 & 2.78 & 0.44 & 6.3 \\
\hline
\end{tabular}

in $\mathrm{PbA}$ collisions can precisely be determined with the mixed-event technique and the large expected amount of like-sign di-leptons in order to reduce uncertainties in the combinatorial-background normalisation. Fig. 52 shows the invariant mass distributions of the di-muon pairs in different rapidity intervals, and Fig. 53 shows the signal-to-background ratios in PbXe collisions at $\sqrt{s_{N N}}=72 \mathrm{GeV}$. Table 21 shows the DY signal and background yields and signal-to-background $(\mathrm{S} / \mathrm{B})$ ratios in these reactions. Overall, the background is significant in the low-mass range $\left(M_{\mu \mu}: 4-5 \mathrm{GeV}\right)$, but it will be suppressed by imposing a stringent cut on the muon transverse momentum. Fig. 53 indicates, that the S/B increases significantly when a single muon cut of $p_{T}^{\mu}>1.2 \mathrm{GeV}$ is applied, compared to the $p_{T}^{\mu}>0.7 \mathrm{GeV}$ case. Nonetheless, while the measurement could be challenging in some kinematic ranges, the expected yields will allow for a definitive test of factorisation of the initial-state effects from $p A$ to $A A$ collisions.

\section{Conclusions}

Unlike the Fermilab-Tevatron and DESY-HERA colliders (with proton beams in the TeV range), no fixed-target programme was planned for the LHC. In this review, we have put forward a strong physics case for such a programme both for the multi-TeV proton and ion LHC beams. Such a physics case relies on extensive theory work and projection studies which have been performed with LHCb- and ALICE-like detectors allowing for high-precision studies in the backward hemisphere of $p p, p A$ and $A A$ collisions.

These projections cover the 3 main research axes of the physics case, namely that of the nucleon and nucleus structure at high momentum fractions, that of the nucleon-spin decomposition in terms of the partonic degrees of freedom and that of the properties of the nuclear matter at extreme conditions such as those resulting from ultra-relativistic heavy-ion collisions.

They are relevant for different possible implementations which we have reviewed including the state-of-the-art solutions provided by modern polarised gas targets or by splitting the beam with a bent crystal. For each of the 


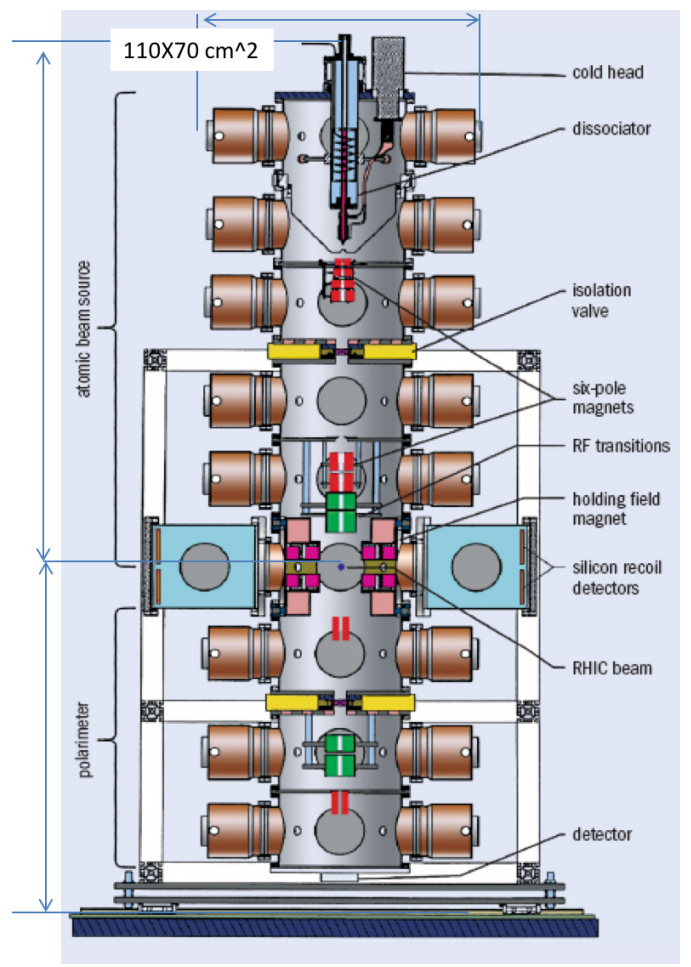

Fig. 54. Schematic view of the polarised H-jet system used at BNL-RHIC collider [103] that consists of an Atomic Beam Source, a turbo-molecular pumping system and a Breit-Rabi polarimeter.

possible implementations, we have also detailed the expected luminosities compatible with the LHCb and ALICE detector capabilities. For a number of studies where such projections are not yet available, we have collected the existing theory predictions for e.g. cross sections, spin and azimuthal asymmetries or nuclear modification factors.

Overall, we believe that the present review constitutes a very solid basis for the elaboration of a rich and fruitful LHC fixed-target programme starting as early as 2020 .

\section{Declaration of competing interest}

The authors declare that they have no known competing financial interests or personal relationships that could have appeared to influence the work reported in this paper.

\section{Acknowledgements}

We thank R. Arnaldi, V. Chambert, F. Fleuret, B. Genolini, V. Kartvelishvili, A. Nass, R. Mikkelsen, S. Platchkov, F. Rathmann, P. Rosier, M. Schmelling, E. Scomparin, E. Steffens, U. Uggerhøj, R. Ulrich, for their involvement at the early stage of this project.

We are particularly grateful to C. Barschel, U. D’Alesio, N. Doshita, M. Ferro-Luzzi, V. Gonçalves, T .Pierog, D. Pitonyak, K. Pressard, M. Schlegel, M. Siddikov, and H. Spiesberger for providing us with material for this review. We thank D. d'Enterria and R. Milner for useful and constructive comments on the manuscript.

We thank F. Arleo, N. Armesto, E. Aschenauer, S. Barsuk, D. Boer, F. Bradamante, M. Calviani, M. Chiosso, Z. Conesa del Valle, J.R. Cudell, J. Cugnon, T. Dahms, A. Dainese, O. Denisov, M. Diehl, B. Espagnon, Y. Gao, M. Gazdzicki, S. Glazov, G. Graziani, A. Holzner, P. Jacobs, J. Jowett, T. Kasemets, M.J. Kim, L. Kluberg, S. Klein, B. Kopeliovich, P. Lenisa, G. Martinez, S. Montesano, P. Mulders, F. Olness, J.C. Peng, B. Pire, C. Pisano, M. Ploskon, A. Poblaguev, J.W. Qiu, B. Saghai, H. Satz, G. Schnell, D. Sivers, T. Stavreva, A. Stocchi, C. Suire, L. Szymanowski, M. Ubiali, T. Ullrich, C. Vallée, R. Vogt, S. Wallon, M. Winn, C. Yin Vallgren, P. Zurita for useful comments and suggestions.

We thank D. Henry for artworks.

This project has received funding from the European Union's Horizon 2020 research and innovation programme under grant agreement STRONG-2020 No. 824093. MGE is supported by the European Research Council (ERC) under the European 


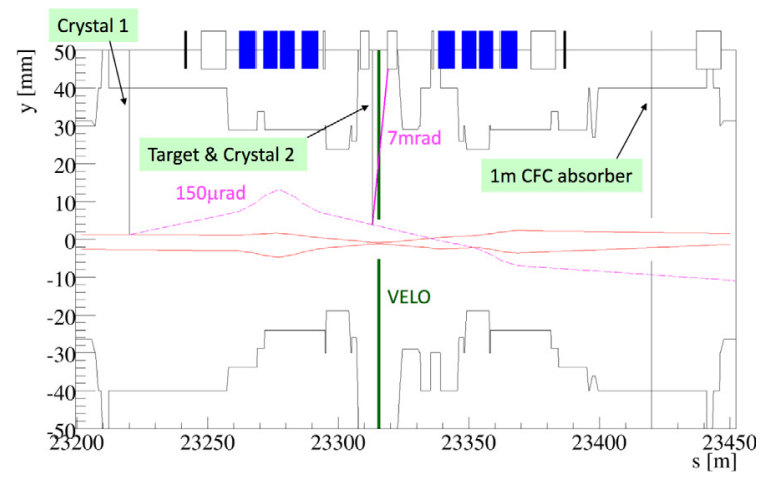

Fig. 55. Possible set-up of the beam-split option upstream of LHCb [From [488]]: a first bent crystal deflects the halo particles upstream the LHCb IP at $5 \sigma$ from the centre line, where $\sigma$ is the beam width. The red full lines represent the main beam envelope. The deflected beam, represented in pink dashed line, is highly focused and its envelope is not visible in the figure. A target inserted in the pipe located in front of the detectors intercepts the deflected halo. For studying electric and magnetic dipole moments of charm charged baryons, a second crystal is necessary. An absorber located downstream $\mathrm{LHCb}$ intercept the halo particles non-interacting with the target.

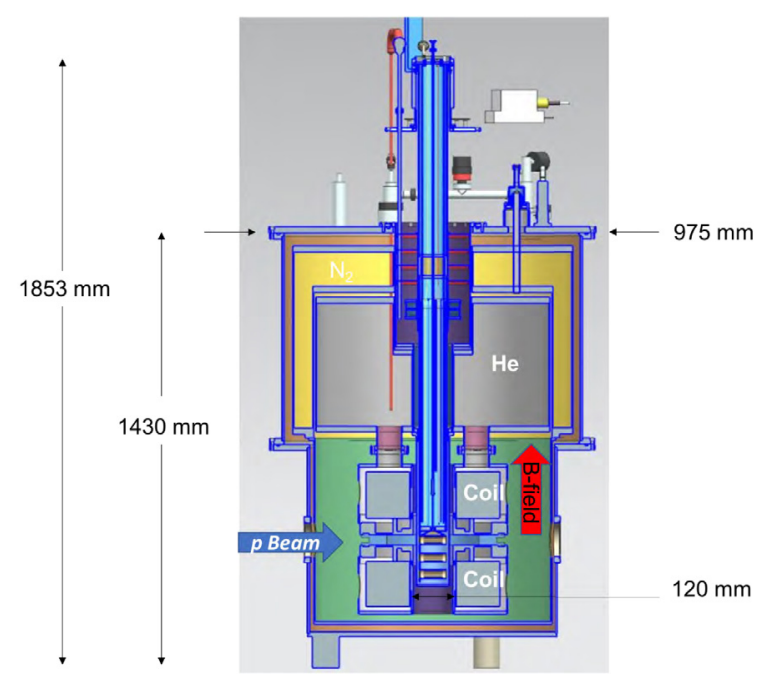

Fig. 56. Schematic view of the E1039 polarised target [77] (cylindrical geometry). It consists of a split coil superconducting magnet, of a refrigerator inside the magnet and in the centre of the system, a target cell, the microwave horn and the NMR coils.

Union's Horizon 2020 research and innovation programme (grant agreement No. 647981, 3DSPIN). AS acknowledges support from U.S. Department of Energy contract DE-AC05-06OR23177, under which Jefferson Science Associates, LLC, manages and operates Jefferson Lab, for his work on this review [JLAB-THY-18-2756]. AS also acknowledges support from the European Commission through the Marie Skłodowska-Curie Action SQuHadron (grant agreement ID: 795475). S.J.B. is supported by the Department of Energy, contract DE-AC02-76SF00515 [SLAC-PUB-17291]. GC acknowledges support from the ERC Ideas Consolidator Grant CRYSBEAM G.A. n. 615089. AK, NT, LM and CH acknowledge support by the RFBR/CNRS grant 18-52-15007 and PRC-1980. ZY is supported by NSFC under the grant number 11575094. This work is supported by Grant No. 2017/26/M/ST2/01074 of the National Science Centre, Poland. The work of JPL, CH and LM was partly supported by the French CNRS via the COPIN-IN2P3 agreement, the IN2P3 project "TMD@NLO" and "GLUE@NLO", the Franco-Spanish PICS "Excitonium", the project Quarkonium4AFTER of the Franco-Chinese LIA FCPPL, by the P2IO Labex via the "Gluodynamics" project and by the Paris-Saclay U. via the P2I Department, that of HSS by the ILP Labex (ANR-11-IDEX0004-02, ANR-10-LABX-63), that of JS by Fundação para a Ciência e a Tecnologia under contract CERN/FIS-PAR/0015/2017, that of EGF by Ministerio de Ciencia e Innovacion of Spain under project FPA2017-83814-P, Unidad de Excelencia Maria de Maetzu under project MDM-2016-0692 and the Paris-Saclay U., that of NY by JSPS Postdoctoral Fellowships for Research Abroad. The work of FD is supported by the "Departments of Excellence 2018-2022" Grant awarded by the Italian Ministry of Education, University and Research (MIUR) (L.232/2016). 


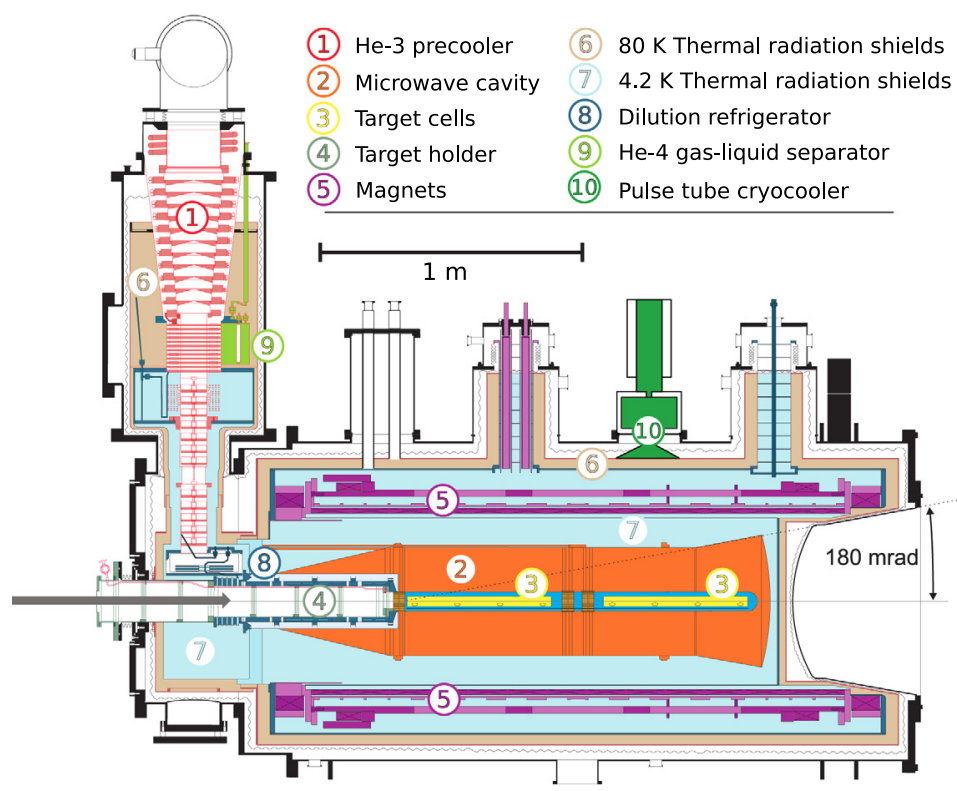

Fig. 57. Schematic side view of the COMPASS polarised target [147-149]. We would like to highlight the two sets of $55 \mathrm{~cm}$ long target cells (denoted (3)) and the $2.5 \mathrm{~T}$ solenoid and $0.6 \mathrm{~T}$ dipole magnets (denoted (5)). The direction of the beam is represented by the grey arrow.
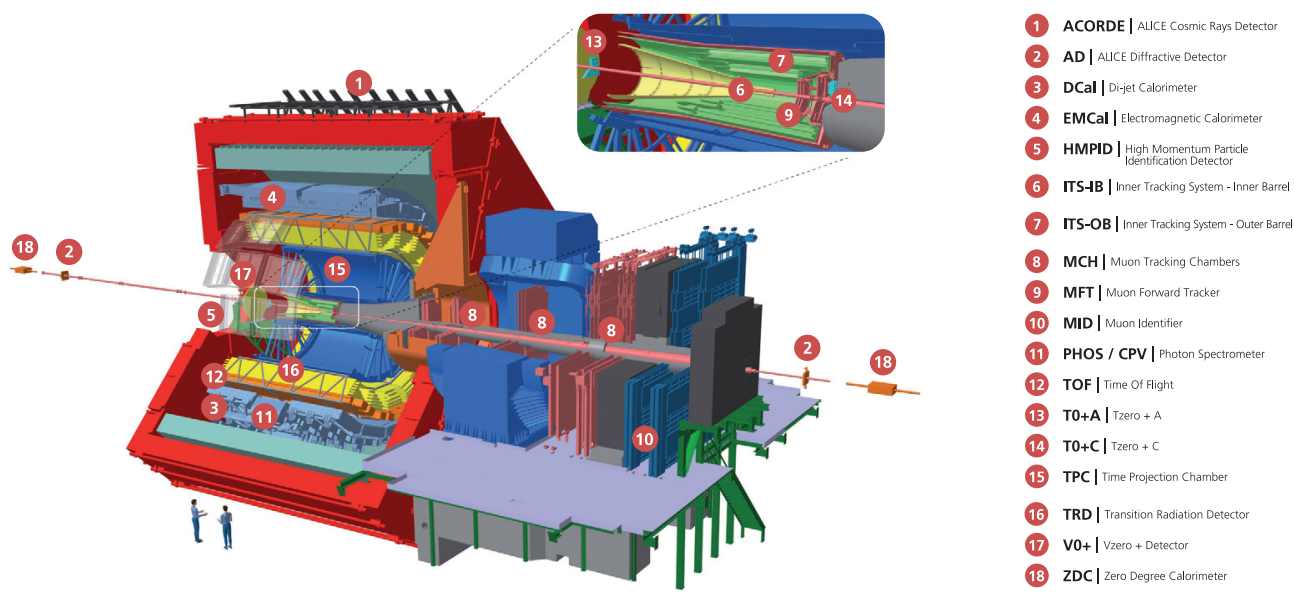

Fig. 58. Schematic view of the ALICE detectors for the LHC Run 3, after the upgrades [161]. Source: Figure courtesy of CERN.

\section{Appendix}

\section{A.1. Schematic view of the H-jet system used at the BNL-RHIC collider}

See Fig. 54.

\section{A.2. Possible setup of the beam-splitted option upstream of $\mathrm{LHCb}$}

See Fig. 55.

\section{A.3. Schematic view of the E1039 target}

See Fig. 56. 


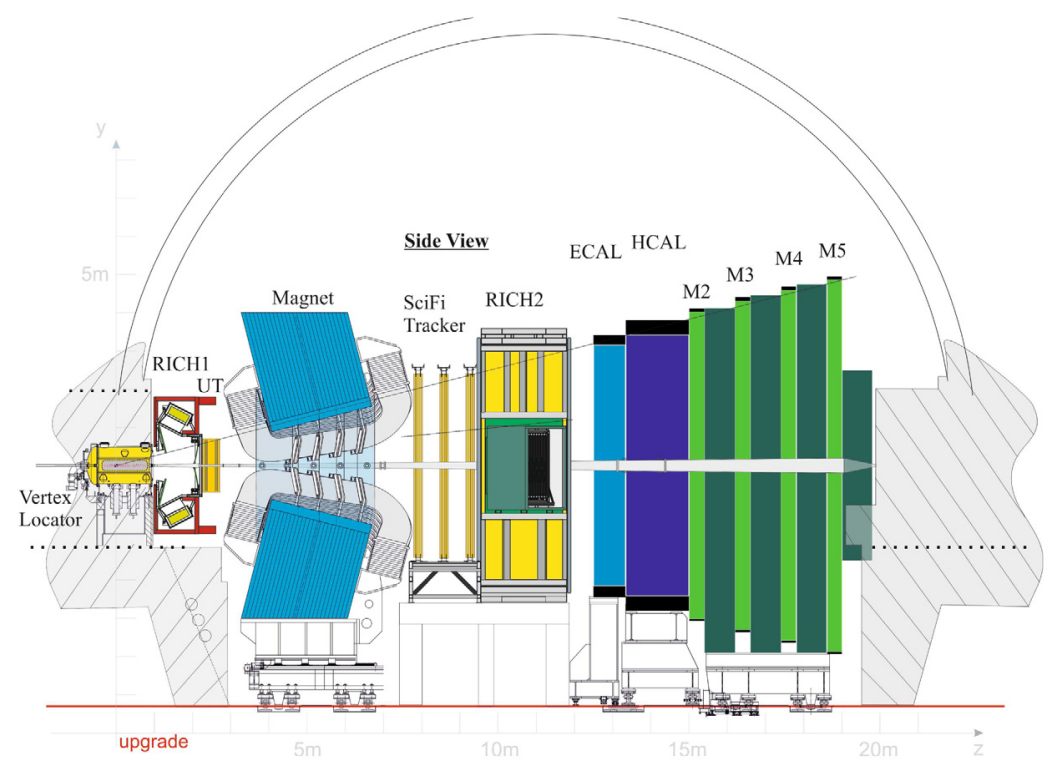

Fig. 59. Schematic view of the LHCb detectors for the LHC Run 3 [164,165]. The main subsystems include the Vertex Locator, the Silicon Micro-strip Upstream Tracker (UT), the Scintillating Fibre (SciFi) Tracker, the Muon Chambers (M2-M5), the Hadron Calorimeter (HCAL), the Electromagnetic Calorimeter (ECAL) and the RICH detectors.

Source: Figure courtesy of CERN.

\section{A.4. Schematic view of the COMPASS target}

See Fig. 57.

\section{A.5. Schematic view of the ALICE detectors}

See Fig. 58.

\section{A.6. Schematic view of the $\mathrm{LHCb}$ detectors}

See Fig. 59.

\section{References}

[1] S.J. Brodsky, F. Fleuret, C. Hadjidakis, J.P. Lansberg, Physics opportunities of a fixed-target experiment using the LHC beams, Phys. Rep. 522 (2013) 239-255, arXiv:1202.6585 [hep-ph].

[2] A. Accardi, et al., Electron-ion collider: The next QCD frontier, Eur. Phys. J. A 52 (9) (2016) 268, http://dx.doi.org/10.1140/epja/i2016-16268-9.

[3] LHeC Study Group Collaboration, J.L. Abelleira Fernandez, et al., A large hadron electron collider at CERN: Report on the physics and design concepts for machine and detector, J. Phys. G 39 (2012) 075001, arXiv:1206.2913 [physics.acc-ph].

[4] European Muon Collaboration, J.J. Aubert, et al., The ratio of the nucleon structure functions $F_{2}^{N}$ for iron and deuterium, Phys. Lett. B 123 (1983) $275-278$.

[5] S. Dulat, T.-J. Hou, J. Gao, M. Guzzi, J. Huston, P. Nadolsky, J. Pumplin, C. Schmidt, D. Stump, C.P. Yuan, New parton distribution functions from a global analysis of quantum chromodynamics, Phys. Rev. D 93 (3) (2016) 033006, arXiv:1506.07443 [hep-ph].

[6] V. Bertone, S. Carrazza, J. Rojo, APFEL: A PDF evolution library with QED corrections, Comput. Phys. Comm. 185 (2014) 1647-1668, arXiv:1310.1394 [hep-ph].

[7] K.J. Eskola, P. Paakkinen, H. Paukkunen, C.A. Salgado, EPPS16: Nuclear parton distributions with LHC data, Eur. Phys. J. C 77 (3) (2017) 163, arXiv: 1612.05741 [hep-ph].

[8] K. Kovarik, et al., nCTEQ15 - Global analysis of nuclear parton distributions with uncertainties in the CTEQ framework, Phys. Rev. D 93 (8) (2016) 085037, arXiv:1509.00792 [hep-ph].

[9] D. de Florian, R. Sassot, P. Zurita, M. Stratmann, Global analysis of nuclear parton distributions, Phys. Rev. D 85 (2012) 074028, arXiv:1112.6324 [hep-ph].

[10] M. Hirai, S. Kumano, T.H. Nagai, Determination of nuclear parton distribution functions and their uncertainties in next-to-leading order, Phys Rev. C 76 (2007) 065207, arXiv:0709.3038 [hep-ph].

[11] L.B. Weinstein, E. Piasetzky, D.W. Higinbotham, J. Gomez, O. Hen, R. Shneor, Short range correlations and the EMC effect, Phys. Rev. Lett. 106 (2011) 052301, arXiv:1009.5666 [hep-ph].

[12] S.J. Brodsky, K.Y.-J. Chiu, J.-P. Lansberg, N. Yamanaka, The gluon and charm content of the deuteron, Phys. Lett. B 783 (2018) 287-293, arXiv: 1805.03173 [hep-ph].

[13] R.L. Jaffe, A. Manohar, The G(1) problem: Fact and fantasy on the spin of the proton, Nuclear Phys. B 337 (1990) $509-546$. 
[14] X.-D. Ji, Gauge-invariant decomposition of nucleon spin, Phys. Rev. Lett. 78 (1997) 610-613, arXiv:hep-ph/9603249 [hep-ph].

[15] E. Leader, C. Lorce, The angular momentum controversy: What's it all about and does it matter? Phys. Rep. 541 (2014) 163-248, arXiv: 1309.4235 [hep-ph].

[16] M. Wakamatsu, Is gauge-invariant complete decomposition of the nucleon spin possible? Internat. J. Modern Phys. A 29 (2014) 1430012, arXiv:1402.4193 [hep-ph].

[17] M. Wakamatsu, Unraveling the physical meaning of the Jaffe-Manohar decomposition of the nucleon spin, Phys. Rev. D 94 (5) (2016) 056004 arXiv:1607.04018 [hep-ph].

[18] M. Burkardt, C.A. Miller, W.D. Nowak, Spin-polarized high-energy scattering of charged leptons on nucleons, Rep. Progr. Phys. 73 (2010) 016201, arXiv:0812.2208 [hep-ph].

[19] European Muon Collaboration, J. Ashman, et al., A measurement of the spin asymmetry and determination of the structure function $g_{1}$ in deep inelastic muon-proton scattering, Phys. Lett. B 206 (1988) 364.

[20] HERMES Collaboration, A. Airapetian, et al., Quark helicity distributions in the nucleon for up, down, and strange quarks from semi-inclusive deep-inelastic scattering, Phys. Rev. D 71 (2005) 012003, arXiv:hep-ex/0407032 [hep-ex].

[21] D. de Florian, R. Sassot, M. Stratmann, W. Vogelsang, Global analysis of helicity parton densities and their uncertainties, Phys. Rev. Lett. 101 (2008) 072001, arXiv:0804.0422 [hep-ph].

[22] COMPASS Collaboration, C. Adolph, et al., The spin structure function $g_{1}^{\mathrm{p}}$ of the proton and a test of the Bjorken sum rule, Phys. Lett. B 753 (2016) 18-28, arXiv:1503.08935 [hep-ex]

[23] HERMES Collaboration, A. Airapetian, et al., Precise determination of the spin structure function g(1) of the proton, deuteron and neutron, Phys. Rev. D 75 (2007) 012007, http://dx.doi.org/10.1103/PhysRevD.75.012007, arXiv:hep-ex/0609039.

[24] STAR Collaboration, L. Adamczyk, et al., Precision measurement of the longitudinal double-spin asymmetry for inclusive jet production in polarized proton collisions at $\sqrt{s}=200 \mathrm{GeV}$, Phys. Rev. Lett. 115 (9) (2015) 092002, arXiv:1405.5134 [hep-ex].

[25] D. de Florian, R. Sassot, M. Stratmann, W. Vogelsang, Evidence for polarization of gluons in the proton, Phys. Rev. Lett. 113 (1) (2014) 012001, arXiv: 1404.4293 [hep-ph].

[26] U. D'Alesio, F. Murgia, Azimuthal and single spin asymmetries in hard scattering processes, Prog. Part. Nucl. Phys. 61 (2008) 394-454, arXiv:0712.4328 [hep-ph].

[27] V. Barone, F. Bradamante, A. Martin, Transverse-spin and transverse-momentum effects in high-energy processes, Prog. Part. Nucl. Phys. 65 (2010) 267-333, arXiv:1011.0909 [hep-ph].

[28] FNAL-E704 Collaboration, D.L. Adams, et al., Analyzing power in inclusive $\pi^{+}$and $\pi^{-}$production at high $x_{F}$ with a $200-G e V$ polarized proton beam, Phys. Lett. B 264 (1991) 462-466.

[29] BRAHMS Collaboration, I. Arsene, et al., Single transverse spin asymmetries of identified charged hadrons in polarized $\mathrm{p}+\mathrm{p}$ collisions at $\sqrt{\mathrm{s}}=$ $62.4 \mathrm{GeV}$, Phys. Rev. Lett. 101 (2008) 042001, arXiv:0801.1078 [nucl-ex].

[30] STAR Collaboration, B.I. Abelev, et al., Forward neutral pion transverse single spin asymmetries in $\mathrm{p}+\mathrm{p}$ collisions at $\sqrt{s}=200 \mathrm{GeV}$, Phys. Rev. Lett. 101 (2008) 222001, arXiv:0801.2990 [hep-ex].

[31] HERMES Collaboration, A. Airapetian, et al., Single-spin asymmetries in semi-inclusive deep-inelastic scattering on a transversely polarized hydrogen target, Phys. Rev. Lett. 94 (2005) 012002, arXiv:hep-ex/0408013 [hep-ex].

[32] COMPASS Collaboration, V. Yu. Alexakhin, et al., First measurement of the transverse spin asymmetries of the deuteron in semi-inclusive deep inelastic scattering, Phys. Rev. Lett. 94 (2005) 202002, arXiv:hep-ex/0503002 [hep-ex].

[33] PHENIX Collaboration, A. Adare, et al., Measurement of transverse single-spin asymmetries for $J / \psi$ production in polarized $p+p$ collisions at $\sqrt{s}=200 \mathrm{GeV}$, Phys. Rev. D 82 (2010) 112008, arXiv:1009.4864 [hep-ex]; Phys. Rev. D 86 (2012) 099904, (erratum).

[34] J. Matoušek, Quarkonium studies at COMPASS experiment, Few Body Syst. 58 (3) (2017) 126.

[35] S.J. Brodsky, D.S. Hwang, I. Schmidt, Initial state interactions and single spin asymmetries in Drell-Yan processes, Nuclear Phys. B 642 (2002) 344-356, arXiv:hep-ph/0206259 [hep-ph].

[36] J.C. Collins, Leading twist single transverse-spin asymmetries: Drell-Yan and deep inelastic scattering, Phys. Lett. B 536 (2002) 43-48, arXiv:hep-ph/0204004 [hep-ph].

[37] S.J. Brodsky, D.S. Hwang, I. Schmidt, Final state interactions and single spin asymmetries in semi-inclusive deep inelastic scattering, Phys. Lett. B 530 (2002) 99-107, arXiv:hep-ph/0201296 [hep-ph].

[38] Y. Koike, W. Vogelsang, F. Yuan, On the relation between mechanisms for single-transverse-spin asymmetries, Phys. Lett. B 659 (2008) 878-884, arXiv:0711.0636 [hep-ph].

[39] A.V. Efremov, O.V. Teryaev, On spin effects in quantum chromodynamics, Sov. J. Nucl. Phys. 36 (1982) $140 ; \quad$ Yad. Fiz. 36 (1982) 242.

[40] A.V. Efremov, O.V. Teryaev, QCD asymmetry and polarized hadron structure functions, Phys. Lett. B 150 (1985) 383.

[41] J.-W. Qiu, G.F. Sterman, Single transverse spin asymmetries, Phys. Rev. Lett. 67 (1991) $2264-2267$.

[42] K. Kanazawa, Y. Koike, A. Metz, D. Pitonyak, Towards an explanation of transverse single-spin asymmetries in proton-proton collisions: the role of fragmentation in collinear factorization, Phys. Rev. D 89 (11) (2014) 111501, arXiv:1404.1033 [hep-ph].

[43] L. Gamberg, Z.-B. Kang, D. Pitonyak, A. Prokudin, Phenomenological constraints on $A_{N}$ in $p^{\uparrow} p \rightarrow \pi X$ from Lorentz invariance relations, Phys. Lett. B 770 (2017) 242-251, arXiv:1701.09170 [hep-ph].

[44] J.C. Collins, D.E. Soper, Back-to-back jets in QCD, Nuclear Phys. B 193 (1981) 381; Nuclear Phys. B 213 (1983) 545, (erratum).

[45] J.C. Collins, D.E. Soper, G.F. Sterman, Transverse momentum distribution in Drell-Yan pair and W and Z Boson production, Nuclear Phys. B 250 (1985) 199-224.

[46] J.C. Collins, D.E. Soper, G.F. Sterman, Factorization of hard processes in QCD, Adv. Ser. Direct. High Energy Phys. 5 (1989) 1-91, arXiv:hepph/0409313 [hep-ph].

[47] X.-D. Ji, J.-P. Ma, F. Yuan, QCD factorization for semi-inclusive deep-inelastic scattering at low transverse momentum, Phys. Rev. D 71 (2005) 034005, arXiv:hep-ph/0404183 [hep-ph].

[48] J. Collins, Foundations of Perturbative QCD, Cambridge University Press, 2013, http://www.cambridge.org/de/knowledge/isbn/item5756723.

[49] M.G. Echevarria, A. Idilbi, I. Scimemi, Factorization theorem for Drell-Yan at low $q_{T}$ and transverse momentum distributions on-the-light-cone, J. High Energy Phys. 07 (2012) 002, arXiv:1111.4996 [hep-ph].

[50] M.G. Echevarria, A. Idilbi, I. Scimemi, Soft and collinear factorization and transverse momentum dependent parton distribution functions, Phys Lett. B 726 (2013) 795-801, arXiv:1211.1947 [hep-ph].

[51] M.G. Echevarria, A. Idilbi, I. Scimemi, Unified treatment of the QCD evolution of all (un-)polarized transverse momentum dependent functions: Collins function as a study case, Phys. Rev. D 90 (1) (2014) 014003, arXiv:1402.0869 [hep-ph].

[52] A. Bacchetta, P.J. Mulders, Deep inelastic leptoproduction of spin-one hadrons, Phys. Rev. D 62 (2000) 114004, arXiv:hep-ph/0007120 [hep-ph].

[53] P.J. Mulders, J. Rodrigues, Transverse momentum dependence in gluon distribution and fragmentation functions, Phys. Rev. D 63 (2001) 094021, arXiv:hep-ph/0009343 [hep-ph].

[54] D. Boer, S. Cotogno, T. van Daal, P.J. Mulders, A. Signori, Y.-J. Zhou, Gluon and Wilson loop TMDs for hadrons of spin $\leq 1$, J. High Energy Phys. 10 (2016) 013, arXiv:1607.01654 [hep-ph]. 
[55] R. Angeles-Martinez, et al., Transverse momentum dependent (TMD) parton distribution functions: status and prospects, Acta Phys. Polon. B 46 (12) (2015) 2501-2534, arXiv:1507.05267 [hep-ph].

[56] D.W. Sivers, Single spin production asymmetries from the hard scattering of point-like constituents, Phys. Rev. D 41 (1990) 83.

[57] D.W. Sivers, Hard scattering scaling laws for single spin production asymmetries, Phys. Rev. D 43 (1991) $261-263$.

[58] J.-W. Qiu, G.F. Sterman, Single transverse spin asymmetries in hadronic pion production, Phys. Rev. D 59 (1999) 014004 , arXiv:hep-ph/9806356 [hep-ph].

[59] N. Hammon, O. Teryaev, A. Schafer, Single spin asymmetry for the Drell-Yan process, Phys. Lett. B 390 (1997) 409-412, arXiv:hep-ph/9611359 [hep-ph].

[60] D. Boer, P.J. Mulders, O.V. Teryaev, Single spin asymmetries from a gluonic background in the Drell-Yan process, Phys. Rev. D 57 (1998) 3057-3064, arXiv:hep-ph/9710223 [hep-ph].

[61] D. Boer, P.J. Mulders, Color gauge invariance in the Drell-Yan process, Nuclear Phys. B 569 (2000) 505-526, arXiv:hep-ph/9906223 [hep-ph].

[62] J.-W. Qiu, G.F. Sterman, Single transverse spin asymmetries in direct photon production, Nuclear Phys. B 378 (1992) $52-78$.

[63] D. Boer, C. Lorce, C. Pisano, J. Zhou, The gluon sivers distribution: status and future prospects, Adv. High Energy Phys. 2015 (2015) 371396, arXiv: 1504.04332 [hep-ph]

[64] PHENIX Collaboration, A. Adare, et al., Measurement of transverse-single-spin asymmetries for midrapidity and forward-rapidity production of hadrons in polarized p+p collisions at $\sqrt{s}=200$ and $62.4 \mathrm{GeV}$, Phys. Rev. D 90 (1) (2014) 012006, arXiv:1312.1995 [hep-ex].

[65] COMPASS Collaboration, C. Adolph, et al., First measurement of the sivers asymmetry for gluons using SIDIS data, Phys. Lett. B 772 (2017) 854-864, arXiv:1701.02453 [hep-ex].

[66] PHENIX Collaboration, C. Aidala, et al., Single-spin asymmetry of $J / \psi$ production in $p+p, p+A l$, and $p+A u$ collisions with transversely polarized proton beams at $\sqrt{s_{N N}}=200 \mathrm{GeV}$, Phys. Rev. D 98 (1) (2018) 012006, arXiv:1805.01491 [hep-ex].

[67] D. Boer, P.J. Mulders, Time reversal odd distribution functions in leptoproduction, Phys. Rev. D 57 (1998) 5780-5786, arXiv:hep-ph/9711485 [hep-ph].

[68] C.S. Lam, W.-K. Tung, A parton model relation sans QCD modifications in lepton pair productions, Phys. Rev. D 21 (1980) 2712.

[69] M. Lambertsen, W. Vogelsang, Drell-Yan lepton angular distributions in perturbative QCD, Phys. Rev. D 93 (11) (2016) 114013, arXiv: 1605.02625 [hep-ph].

[70] D. Boer, C. Pisano, Polarized gluon studies with charmonium and bottomonium at LHCb and AFTER, Phys. Rev. D 86 (2012) 094007, arXiv:1208.3642 [hep-ph].

[71] A. Signori, Gluon TMDs in quarkonium production, Few Body Syst. 57 (8) (2016) 651-655, arXiv:1602.03405 [hep-ph].

[72] W.J. den Dunnen, J.P. Lansberg, C. Pisano, M. Schlegel, Accessing the transverse dynamics and polarization of gluons inside the proton at the LHC, Phys. Rev. Lett. 112 (2014) 212001, arXiv:1401.7611 [hep-ph].

[73] J.P. Lansberg, et al., Spin physics and TMD studies at a fixed-target experiment at the LHC (AFTER@LHC), EPJ Web Conf. 85 (2015) 02038 arXiv:1410.1962 [hep-ex].

[74] M.G.A. Buffing, A. Mukherjee, P.J. Mulders, Generalized universality of higher transverse moments of quark TMD correlators, Phys. Rev. D 86 (2012) 074030, arXiv:1207.3221 [hep-ph].

[75] M.G.A. Buffing, A. Mukherjee, P.J. Mulders, Generalized universality of definite rank gluon transverse momentum dependent correlators, Phys Rev. D 88 (2013) 054027, arXiv:1306.5897 [hep-ph].

[76] M.G.A. Buffing, Color and TMD Universality in Hadronic Interactions (Ph.D. thesis), NIKHEF, Amsterdam, 2015, http://www.nikhef.nl/pub/ services/biblio/theses_pdf/thesis_M_Buffing.pdf.

[77] A. Klein, et al., Letter of intent for a Drell-Yan experiment with a polarized proton target, 2013, FERMILAB-LOI-2013-01.

[78] COMPASS Collaboration, C. Quintans, Future Drell-Yan measurements in COMPASS, J. Phys. Conf. Ser. 295 (2011) 012163.

[79] STAR Collaboration, L. Adamczyk, et al., Measurement of the transverse single-spin asymmetry in $p^{\uparrow}+p \rightarrow W^{ \pm} / Z^{0}$ at RHIC, Phys. Rev. Lett. 116 (13) (2016) 132301, arXiv:1511.06003 [nucl-ex].

[80] E.-C. Aschenauer, et al., The RHIC Cold QCD Plan for 2017 to 2023: A Portal to the EIC, arXiv:1602.03922 [nucl-ex].

[81] COMPASS Collaboration, M. Aghasyan, et al., First measurement of transverse-spin-dependent azimuthal asymmetries in the Drell-Yan process, Phys. Rev. Lett. 119 (11) (2017) 112002, arXiv:1704.00488 [hep-ex].

[82] D. Boer, P.J. Mulders, F. Pijlman, Universality of T odd effects in single spin and azimuthal asymmetries, Nuclear Phys. B 667 (2003) 201-241, arXiv:hep-ph/0303034 [hep-ph].

[83] X.-D. Ji, J.-W. Qiu, W. Vogelsang, F. Yuan, A unified picture for single transverse-spin asymmetries in hard processes, Phys. Rev. Lett. 97 (2006) 082002, arXiv:hep-ph/0602239 [hep-ph].

[84] X.-D. Ji, J.-W. Qiu, W. Vogelsang, F. Yuan, Single transverse-spin asymmetry in Drell-Yan production at large and moderate transverse momentum, Phys. Rev. D 73 (2006) 094017, arXiv:hep-ph/0604023 [hep-ph].

[85] X.-D. Ji, J.-W. Qiu, W. Vogelsang, F. Yuan, Single-transverse spin asymmetry in semi-inclusive deep inelastic scattering, Phys. Lett. B 638 (2006) 178-186, arXiv:hep-ph/0604128 [hep-ph].

[86] I. Karpenko, Rapidity scan in heavy ion collisions at $\sqrt{s_{\mathrm{NN}}}=72 \mathrm{GeV}$ using a viscous hydro + cascade model, Acta Phys. Polon. B 50 (2019) 141-148, arXiv:1805.11998 [nucl-th].

[87] F. Becattini, J. Cleymans, Chemical equilibrium in heavy ion collisions: Rapidity dependence, J. Phys. G 34 (2007) S959-964, arXiv:hepph/0701029 [hep-ph].

[88] V. Begun, D. Kikola, V. Vovchenko, D. Wielanek, Estimation of the freeze-out parameters reachable in a fixed-target experiment at the CERN Large Hadron Collider, Phys. Rev. C 98 (3) (2018) 034905, arXiv:1806.01303 [nucl-th].

[89] STAR Collaboration, M.M. Aggarwal, et al. An Experimental Exploration of the QCD Phase Diagram: The Search for the Critical Point and the Onset of De-confinement, arXiv:1007.2613 [nucl-ex].

[90] STAR Collaboration, L. Adamczyk, et al., Bulk properties of the medium produced in relativistic heavy-ion collisions from the beam energy scan program, Phys. Rev. C 96 (4) (2017) 044904, arXiv:1701.07065 [nucl-ex].

[91] G. Denicol, A. Monnai, B. Schenke, Moving forward to constrain the shear viscosity of QCD matter, Phys. Rev. Lett. 116 (21) (2016) 212301, arXiv: 1512.01538 [nucl-th].

[92] B. Schenke, Bulk evolution of heavy ion collisions in the beam energy scan: New developments and first results, in Quark Matter 2015, 27 September - 3 October, 2015, Kobe, Japan.

[93] C. Lourenco, H.K. Wohri, Heavy flavour hadro-production from fixed-target to collider energies, Phys. Rep. 433 (2006) 127-180, arXiv:hepph/0609101 [hep-ph].

[94] R. Rapp, D. Blaschke, P. Crochet, Charmonium and bottomonium production in heavy-ion collisions, Prog. Part. Nucl. Phys. 65 (2010) 209-266, arXiv:0807.2470 [hep-ph].

[95] A. Andronic, et al., Heavy-flavour and quarkonium production in the LHC era: from proton-proton to heavy-ion collisions, Eur. Phys. J. C 76 (3) (2016) 107, arXiv:1506.03981 [nucl-ex].

[96] L. Kluberg, 20 years of J/psi suppression at the CERN SPS: Results from experiments NA38, NA51 and NA50, Eur. Phys. J. C 43 (2005) 145-156. 
[97] PHENIX Collaboration, K. Adcox, et al., Formation of dense partonic matter in relativistic nucleus-nucleus collisions at RHIC: Experimental evaluation by the PHENIX collaboration, Nuclear Phys. A 757 (2005) 184-283, arXiv:nucl-ex/0410003 [nucl-ex].

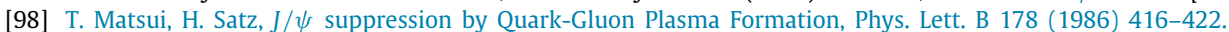

[99] M. Ferro-Luzzi, Proposal for an absolute luminosity determination in colliding beam experiments using vertex detection of beam-gas interactions, Nucl. Instrum. Methods A 553 (2005) 388-399.

[100] R. Aaij, et al., Performance of the LHCb vertex locator, J. Instrum. 9 (2014) P09007, arXiv:1405.7808 [physics.ins-det].

[101] LHCb Collaboration, E. Maurice, Fixed-target physics at LHCb, in: 5th Large Hadron Collider Physics Conference (LHCP 2017) Shanghai, China, 2017, 15-20. 2017, 2017, arXiv:1708.05184 [hep-ex]. http://inspirehep.net/record/1616496/files/arXiv:1708.05184.pdf.

[102] E. Steffens, W. Haeberli, Polarized gas targets, Rep. Progr. Phys. 66 (11) (2003) 1887, http://stacks.iop.org/0034-4885/66/i=11/a=R02.

[103] A. Zelenski, et al., Absolute polarized H-jet polarimeter development, for RHIC, Nucl. Instrum. Methods A 536 (2005) $248-254$.

[104] W. Scandale, et al., Observation of channeling for $6500 \mathrm{GeV} /$ c protons in the crystal assisted collimation setup for LHC, Phys. Lett. B 758 (2016) 129-133.

[105] S. Montesano, et al., Testing the double-crystal setup for physics beyond collider experiments in the UA9-SPS experiment, in: Proceedings, The 9th International Particle Accelerator Conference (IPAC2018), Vancouver, BC, Canada, IPAC2018, p. TUPAF043, 2018.

[106] A.B. Kurepin, N.S. Topilskaya, M.B. Golubeva, Charmonium production in fixed-target experiments with SPS and LHC beams at CERN, Phys. Atom. Nucl. 74 (2011) 446-452; Yad. Fiz. 74 (2011) 467.

[107] K. Ehret, Commissioning of the HERA-B internal target: Using the HERA proton ring as a B factory, Nucl. Instrum. Methods A 446 (2000) $190-198$

[108] STAR Collaboration, K.C. Meehan, Fixed target collisions at STAR, Nuclear Phys. A 956 (2016) 878-881.

[109] O.S. Bruning, P. Collier, P. Lebrun, S. Myers, R. Ostojic, J. Poole, P. Proudlock, LHC Design Report 1: The LHC Main Ring. CERN-2004-003-V1, CERN-2004-003, CERN-2004-003-V-1, 2004

[110] M. Benedikt, P. Collier, V. Mertens, J. Poole, K. Schindl, LHC Design Report. 3. The LHC injector chain. CERN-2004-003-V-3, CERN-2004-003.

[111] Spin Muon Collaboration, D. Adams, et al., The polarized double cell target of the SMC, Nucl. Instrum. Methods A 437 (1999) $23-67$.

[112] C. Barschel, Precision Luminosity Measurement At LHCb with Beam-Gas Imaging (Ph.D. thesis), RWTH Aachen U., 2014, http://inspirehep.net/ record/1339684/files/CERN-THESIS-2013-301.pdf.

[113] LHCb Collaboration, R. Aaij, et al., Precision luminosity measurements at LHCb, J. Instrum. 9 (12) (2014) P12005, arXiv:1410.0149 [hep-ex].

[114] T. Pierog, I. Karpenko, J.M. Katzy, E. Yatsenko, K. Werner, EPOS LHC: Test of collective hadronization with data measured at the CERN Large Hadron Collider, Phys. Rev. C 92 (3) (2015) 034906, arXiv:1306.0121 [hep-ph].

[115] A. Ferrari, P.R. Sala, A. Fasso, J. Ranft, FLUKA: A multi-particle transport code (Program version 2005). CERN-2005-010, SLAC-R-773, INFN-TC-05-11.

[116] LHCb Collaboration, R. Aaij, et al., Measurement of antiproton production in pHe collisions at $\sqrt{s_{N N}}=110 \mathrm{GeV}$, Phys. Rev. Lett. 121 (22) (2018) 222001, arXiv:1808.06127 [hep-ex].

[117] LHCb Collaboration, Measurement of $J / \psi$ and $D^{0}$ production in pAr collisions at $\sqrt{s_{N N}}=110 \mathrm{GeV}$, https://cds.cern.ch/record/2255650. LHCb-CONF-2017-001, CERN-LHCb-CONF-2017-001.

[118] A. Zelenski, Personal communication.

[119] H. Okada, et al., Measurement of the analyzing power in pp elastic scattering in the peak CNI region at RHIC, Phys. Lett. B 638 (2006) 450-454, arXiv:nucl-ex/0502022 [nucl-ex].

[120] A. Poblaguev, et al., The HJET polarimeter in RHIC run 2017, PoS PSTP2017 (2017) 022.

[121] C. Barschel, P. Lenisa, A. Nass, E. Steffens, A gas target internal to the LHC for the study of pp single-spin asymmetries and heavy ion collisions Adv. High Energy Phys. 2015 (2015) 463141.

[122] M. Mikirtychyants, et al., The polarized H and D atomic beam source for ANKE at COSY-Julich, Nucl. Instrum. Methods A 721 (2013) $83-98$, arXiv:1212.1840 [physics.ins-det].

[123] K. Grigoryev, et al., Machine studies for the development of storage cells at the ANKE facility of COSY, Nucl. Instrum. Methods A 599 (2009) $130-139$.

[124] G. Ciullo, M. Statera, P. Lenisa, A. Nass, G. Tagliente, The H and D polarized target for spin-filtering measurements at COSY, Int. J. Mod. Phys. Conf. Ser. 40 (2016) 1660149.

[125] W.A. Dezarn, et al., Polarized internal gas target for hydrogen and deuterium at the IUCF cooler ring, Nucl. Instrum. Methods A 362 (1995) $36-40$.

[126] HERMES Collaboration, A. Airapetian, et al., The HERMES polarized hydrogen and deuterium gas target in the HERA electron storage ring, Nucl. Instrum. Methods A 540 (2005) 68-101, arXiv:physics/0408137 [physics].

[127] P. and Di Nezza, Polarized fixed target at LHC, in: Talk at Physics Beyond Collider, Annual Workshop, 2017 November 21-22.

[128] J. Jowett, H. Braun, M. Gresham, E. Mahner, A. Nicholson, E. Shaposhnikova, Limits to the performance of the LHC with ion beams, in: EPAC2004 2004.

[129] J. Jowett, Personal communication.

[130] G. Arduini, K. Elsener, G. Fidecaro, M. Gyr, W. Herr, J. Klem, U.I. Uggerhoj, E. Weisse, On the energy dependence of proton beam extraction with a bent crystal, Phys. Lett. B 422 (1998) 325-333.

[131] A. Asseev, et al., First observation of luminosity driven extraction using channeling with a bent crystal, Phys. Rev. ST Accel. Beams 1 (1998) 022801.

[132] A.G. Afonin, et al., Observation and comparative analysis of proton beam extraction or collimation by different planar channels of a bent crystal, Phys. Rev. ST Accel. Beams 15 (2012) 081001.

[133] W. Scandale, et al., Comparative results on collimation of the SPS beam of protons and Pb ions with bent crystals, Phys. Lett. B 703 (2011) 547-551.

[134] W. Scandale, et al., First results on the SPS beam collimation with bent crystals, Phys. Lett. B 692 (2010) 78-82.

[135] W. Scandale, et al., Strong reduction of the off-momentum halo in crystal assisted collimation of the SPS beam, Phys. Lett. B 714 (2012) 231-236.

[136] V.M. Biryukov, Yu. A. Chesnokov, V.I. Kotov, V. Guidi, C. Malagu, G. Martinelli, M. Stefancich, D. Vincenzi, W. Scandale, Crystal deflector for highly efficient channeling extraction of a proton beam from accelerators, Rev. Sci. Instrum. 73 (2002) 3170-3173.

[137] W. Scandale, et al., Comprehensive study of beam focusing by crystal devices, Phys. Rev. Accel. Beams 21 (1) (2018) 014702.

[138] A. Baurichter, et al., Channeling of high-energy particles in bent crystals: Experiments at the CERN SPS, Nucl. Instrum. Methods B 164-165 (2000) 27-43.

[139] W. Scandale, Experimental insertions for the LHC, in: ECFA Large Hadron Collider (LHC) Workshop: Physics and Instrumentation Aachen, Germany, (1990) October 4-9, 1990, pp. 760-764.

[140] E. Uggerhoj, U.I. Uggerhj, Strong crystalline fields: A possibility for extraction from the LHC, Nucl. Instrum. Methods B 234 (2005) 31-39.

[141] http://crysbeam.roma1.infn.it/index.html. 
[142] A. Mazzolari, M. Romagnoni, R. Camattari, E. Bagli, L. Bandiera, G. Germogli, V. Guidi, G. Cavoto, Bent crystals for efficient beam steering of multi tev-particle beams, Eur. Phys. J. C 78 (9) (2018) 720.

[143] A.S. Fomin, et al., Feasibility of measuring the magnetic dipole moments of the charm baryons at the LHC using bent crystals, J. High Energy Phys. 08 (2017) 120, arXiv:1705.03382 [hep-ph].

[144] E. Bagli, et al., Electromagnetic dipole moments of charged baryons with bent crystals at the LHC, Eur. Phys. J. C 77 (12) (2017) 828, arXiv: 1708.08483 [hep-ex].

[145] F.J. Botella, L.M. Garcia Martin, D. Marangotto, F.M. Vidal, A. Merli, N. Neri, A. Oyanguren, J.R. Vidal, On the search for the electric dipole moment of strange and charm baryons at LHC, Eur. Phys. J. C 77 (3) (2017) 181, arXiv:1612.06769 [hep-ex].

[146] C. Barschel, et al., Report from the LHC Fixed Target Working Group of the CERN Physics beyond Colliders Forum, Tech. Rep. CERN-PBC-REPORT-2019-001, CERN, Geneva, 2019, https://cds.cern.ch/record/2653780.

[147] COMPASS Collaboration, M. Pesek, J. Matousek, COMPASS Polarized target for Drell-Yan, PoS PSTP 2013 (2013) 009.

[148] N. Doshita, et al., Performance of the COMPASS polarized target dilution refrigerator, Nucl. Instrum. Methods A 526 (2004) 138-143.

[149] J. Ball, et al., First results of the large COMPASS 6lid polarized target, Nucl. Instrum. Methods A 498 (2003) $101-111$.

[150] S. Goertz, W. Meyer, G. Reicherz, H. Polarized, D and He-3 targets for particle physics experiments, Prog. Part. Nucl. Phys. 49 (2002) 403-489; Prog. Part. Nucl. Phys. 51 (2003) 309, (erratum).

[151] K. Kondo, et al., Polarization measurement in the COMPASS polarized target, Nucl. Instrum. Methods A 526 (2004) $70-75$.

[152] ALICE Collaboration, K. Aamodt, et al., The ALICE experiment at the CERN LHC, J. Instrum. 3 (2008) S08002.

[153] LHCb Collaboration, A.A. Alves Jr., et al., The LHCb detector at the LHC, J. Instrum. 3 (2008) S08005.

[154] M. Anderson, et al., The star time projection chamber: A unique tool for studying high multiplicity events at RHIC, Nucl. Instrum. Methods A 499 (2003) 659-678, arXiv:nucl-ex/0301015 [nucl-ex].

[155] STAR Collaboration, M. Beddo, et al., The STAR barrel electromagnetic calorimeter, Nucl. Instrum. Methods A 499 (2003) $725-739$.

[156] STAR Collaboration, C.E. Allgower, et al., The STAR endcap electromagnetic calorimeter, Nucl. Instrum. Methods A 499 (2003) 740-750.

[157] W.W. Jacobs, the STAR Collaboration, STAR Calorimetry, J. Phys. Conf. Ser. 160 (2009) 012008, http://dx.doi.org/10.1088/1742-6596/160/1/ 012008.

[158] PHENIX Collaboration, K. Adcox, et al., PHENIX detector overview, Nucl. Instrum. Methods A 499 (2003) 469-479.

[159] K. Werner, F.-M. Liu, T. Pierog, Parton ladder splitting and the rapidity dependence of transverse momentum spectra in deuteron-gold collisions at RHIC, Phys. Rev. C 74 (2006) 044902, arXiv:hep-ph/0506232 [hep-ph].

[160] ALICE Collaboration, B.B. Abelev, et al., Performance of the ALICE experiment at the CERN LHC, Internat. J. Modern Phys. A 29 (2014) 1430044, arXiv: 1402.4476 [nucl-ex].

[161] B. Abelev, et al., Upgrade of the alice experiment: Letter of intent, J. Phys. G: Nucl. Part. Phys. 41 (8) (2014) 087001.

[162] F. Galluccio, et al., Physics opportunities for a fixed-target programme in the ALICE experiment, http://cds.cern.ch/record/2671944. CERN-PBC-Notes-2019-004.

[163] LHCb Collaboration, R. Aaij, et al., LHCB detector performance, Internat. J. Modern Phys. A 30 (07) (2015) 1530022, arXiv:1412.6352 [hep-ex].

[164] LHCb Collaboration, LHCb VELO Upgrade Technical Design Report, Tech. Rep. CERN-LHCC-2013-021. LHCB-TDR-013, 2013, http://cds.cern.ch/ record/1624070.

[165] LHCb Collaboration, LHCb Tracker Upgrade Technical Design Report, Tech. Rep. CERN-LHCC-2014-001. LHCB-TDR-015, 2014, http://cds.cern. ch/record/1647400.

[166] LHCb Collaboration, R. Aaij, et al., Expression of Interest for a Phase-II LHCb Upgrade: Opportunities in Flavour Physics, and beyond, in the HL-LHC Era, Tech. Rep., CERN-LHCC-2017-003, CERN, Geneva, 2017, https://cds.cern.ch/record/2244311.

[167] K.C. Akiba, et al., The HeRSCheL detector: high-rapidity shower counters for LHCb, J. Instrum. 13 (04) (2018) P04017, arXiv:1801.04281 [physics.ins-det].

[168] LHCb Collaboration, R. Aaij, et al., First measurement of charm production in its fixed-target configuration at the LHC, Phys. Rev. Lett. 122 (13) (2019) 132002, arXiv:1810.07907 [hep-ex].

[169] P. Di Nezza, et al., The SMOG2 project, http://cds.cern.ch/record/2651269. CERN-PBC-Notes-2018-007.

[170] LHCb Collaboration, LHCb SMOG Upgrade. https://cds.cern.ch/record/2673690.

[171] G. Graziani, LHCb as a fixed target experiment. Physics Beyond Collider, General Meeting, November 5, 2019.

[172] C.A. Aidala, et al., The LHCSpin Project, arXiv:1901.08002 [hep-ex].

[173] L.L. Pappalardo, LHCspin. Talk at FTE@LHC \& NLOAccess STRONG2020 joint kick-off meeting, November 7-8, 2019.

[174] J. Gao, M. Guzzi, J. Huston, H.-L. Lai, Z. Li, P. Nadolsky, J. Pumplin, D. Stump, C.P. Yuan, CT10 next-to-next-to-leading order global analysis of QCD, Phys. Rev. D 89 (3) (2014) 033009, arXiv:1302.6246 [hep-ph].

[175] NNPDF Collaboration, R.D. Ball, et al., Parton distributions for the LHC run II, J. High Energy Phys. 04 (2015) 040, arXiv:1410.8849 [hep-ph].

[176] L.A. Harland-Lang, A.D. Martin, P. Motylinski, R.S. Thorne, Parton distributions in the LHC era: MMHT 2014 PDFs, Eur. Phys. J. C 75 (5) (2015) 204, arXiv:1412.3989 [hep-ph].

[177] S. Alekhin, J. Blumlein, S. Moch, The ABM parton distributions tuned to LHC data, Phys. Rev. D 89 (5) (2014) 054028, arXiv:1310.3059 [hep-ph].

[178] J.F. Owens, A. Accardi, W. Melnitchouk, Global parton distributions with nuclear and finite-Q ${ }^{2}$ corrections, Phys. Rev. D 87 (9) (2013) 094012, arXiv:1212.1702 [hep-ph].

[179] P. Jimenez-Delgado, E. Reya, Delineating parton distributions and the strong coupling, Phys. Rev. D 89 (7) (2014) 074049, arXiv:1403.1852 [hep-ph].

[180] G. Moreno, et al., Dimuon production in proton - copper collisions at $\sqrt{s}=38.8$ GeV, Phys. Rev. D 43 (1991) $2815-2836$.

[181] NuSea Collaboration and J. C. Webb, others, Absolute Drell-Yan dimuon cross-sections in 800 GeV / c pp and pd collisions, arXiv:hepex/0302019 [hep-ex]. FERMILAB-PUB-03-302-E.

[182] J.C. Webb, Measurement of Continuum Dimuon Production in 800-GeV/C Proton Nucleon Collisions (Ph.D. thesis), New Mexico State U., 2003, arXiv:hep-ex/0301031 [hep-ex]. http://lss.fnal.gov/cgi-bin/find_paper.pl?thesis-2002-56.

[183] NuSea Collaboration, R.S. Towell, et al., Improved measurement of the anti-d / anti-u asymmetry in the nucleon sea, Phys. Rev. D 64 (2001) 052002, arXiv:hep-ex/0103030 [hep-ex].

[184] O. Nachtmann, Inequalities for structure functions of deep inelastic lepton-nucleon scattering giving tests of basic algebraic structures, Nuclear Phys. B 38 (1972) 397-417.

[185] S.J. Brodsky, G.R. Farrar, Scaling laws at large transverse momentum, Phys. Rev. Lett. 31 (1973) $1153-1156$.

[186] R.D. Ball, E.R. Nocera, J. Rojo, The asymptotic behaviour of parton distributions at small and large $x$, Eur. Phys. J. C 76 (7) (2016) 383, arXiv: 1604.00024 [hep-ph].

[187] A. Accardi, L.T. Brady, W. Melnitchouk, J.F. Owens, N. Sato, Constraints on large- $x$ parton distributions from new weak boson production and deep-inelastic scattering data, Phys. Rev. D 93 (11) (2016) 114017, arXiv:1602.03154 [hep-ph].

[188] F.E. Close, $v W^{2}$ At small $\omega^{\prime}$ and resonance form-factors in a quark model with broken SU(6), Phys. Lett. 43B (1973) 422-426

[189] G.R. Farrar, D.R. Jackson, Pion and nucleon structure functions near x=1, Phys. Rev. Lett. 35 (1975) 1416. 
[190] W. Melnitchouk, A.W. Thomas, Neutron / proton structure function ratio at large x, Phys. Lett. B 377 (1996) 11-17, arXiv:nucl-th/9602038 [nucl-th].

[191] F.E. Close, W. Melnitchouk, Symmetry breaking and quark-hadron duality in structure functions, Phys. Rev. C 68 (2003) 035210, arXiv:hepph/0302013 [hep-ph].

[192] R.J. Holt, C.D. Roberts, Distribution functions of the nucleon and pion in the valence region, Rev. Modern Phys. 82 (2010) 2991-3044 arXiv: 1002.4666 [nucl-th].

[193] C.D. Roberts, R.J. Holt, S.M. Schmidt, Nucleon spin structure at very high-x, Phys. Lett. B 727 (2013) 249-254, arXiv:1308.1236 [nucl-th].

[194] H. Paukkunen, P. Zurita, PDF reweighting in the hessian matrix approach, J. High Energy Phys. 12 (2014) 100, arXiv:1402.6623 [hep-ph]

[195] S. Alekhin, et al., Herafitter, Eur. Phys. J. C 75 (7) (2015) 304, arXiv:1410.4412 [hep-ph].

[196] R. Boughezal, J.M. Campbell, R.K. Ellis, C. Focke, W. Giele, X. Liu, F. Petriello, C. Williams, Color singlet production at NNLO in MCFM, Eur. Phys. J. C 77 (1) (2017) 7, arXiv:1605.08011 [hep-ph].

[197] R. Gavin, Y. Li, F. Petriello, S. Quackenbush, W physics at the LHC with FEWZ 2.1, Comput. Phys. Comm. 184 (2013) 208-214, arXiv:1201.5896 [hep-ph].

[198] Fermilab E906 Collaboration, Paul E. Reimer, Measuring the flavor asymmetry in the sea quarks of the proton, in: Proceedings, 18th International Workshop on Deep-inelastic scattering and related subjects (DIS 2010): Florence, Italy, April 19-23, 2010, PoS DIS2010 (2010) 277, http://dx.doi.org/10.22323/1.106.0277.

[199] T. Jezo, M. Klasen, D.R. Lamprea, F. Lyonnet, I. Schienbein, NLO+NLL limits on $W^{\prime}$ and $Z^{\prime}$ gauge boson masses in general extensions of the Standard Model, J. High Energy Phys. 12 (2014) 092, arXiv:1410.4692 [hep-ph].

[200] R. Bonciani, T. Jezo, M. Klasen, F. Lyonnet, I. Schienbein, Electroweak top-quark pair production at the LHC with $Z^{\prime}$ bosons to NLO QCD in POWHEG, J. High Energy Phys. 02 (2016) 141, arXiv:1511.08185 [hep-ph].

[201] F. Lyonnet, A. Kusina, T. Jezo, K. Kovarík, F. Olness, I. Schienbein, J.-Y. Yu, On the intrinsic bottom content of the nucleon and its impact on heavy new physics at the LHC, J. High Energy Phys. 07 (2015) 141, arXiv:1504.05156 [hep-ph].

[202] S.J. Brodsky, J.C. Collins, S.D. Ellis, J.F. Gunion, A.H. Mueller, Intrinsic Chevrolets at the SSC, in: Electroweak Symmetry Breaking. Proceedings, Workshop, Berkeley, USA, June 3-22, 1984. http://www-public.slac.stanford.edu/sciDoc/docMeta.aspx?slacPubNumber=SLAC-PUB-15471.

[203] S.J. Brodsky, P. Hoyer, C. Peterson, N. Sakai, The intrinsic charm of the proton, Phys. Lett. B 93 (1980) 451-455.

[204] M. Franz, M.V. Polyakov, K. Goeke, Heavy quark mass expansion and intrinsic charm in light hadrons, Phys. Rev. D 62 (2000) 074024 , arXiv:hep-ph/0002240 [hep-ph].

[205] S.J. Brodsky, A. Kusina, F. Lyonnet, I. Schienbein, H. Spiesberger, R. Vogt, A review of the intrinsic heavy quark content of the nucleon, Adv. High Energy Phys. 2015 (2015) 231547, arXiv:1504.06287 [hep-ph].

[206] European Muon Collaboration, J.J. Aubert, et al., Production of charmed particles in $250 \mathrm{GeV} \mu^{+}$- iron interactions, Nuclear Phys. B 213 (1983) 31-64.

[207] B.W. Harris, J. Smith, R. Vogt, Reanalysis of the EMC charm production data with extrinsic and intrinsic charm at NLO, Nuclear Phys. B 461 (1996) 181-196, arXiv:hep-ph/9508403 [hep-ph].

[208] NNPDF Collaboration, R.D. Ball, V. Bertone, M. Bonvini, S. Carrazza, S. Forte, A. Guffanti, N.P. Hartland, J. Rojo, L. Rottoli, A determination of the charm content of the proton, Eur. Phys. J. C 76 (11) (2016) 647, arXiv:1605.06515 [hep-ph].

[209] R608 Collaboration, P. Chauvat, et al., Production of $\Lambda_{c}$ with large $x_{F}$ at the ISR, Phys. Lett. B 199 (1987) 304.

[210] SELEX Collaboration, F.G. Garcia, et al., Hadronic production of $\Lambda_{c}$ from $600-\mathrm{GeV} / \mathrm{c} \pi^{-}, \Sigma^{-}$and $p$ beams, Phys. Lett. B 528 (2002) 49-57, arXiv:hep-ex/0109017 [hep-ex].

[211] S. Groote, S. Koshkarev, Production of doubly charmed baryons nearly at rest, Eur. Phys. J. C 77 (8) (2017) 509, arXiv:1704.02850 [hep-ph].

[212] S. Koshkarev, S. Groote, Double quarkonium production at high feynman-x, Nuclear Phys. B 915 (2017) 384-391, arXiv:1611.08149 [hep-ph].

[213] S. Koshkarev, Production of the Doubly Heavy Baryons, $B_{c}$ Meson and the All-charm Tetraquark at AFTER@LHC with Double Intrinsic Heavy Mechanism, arXiv:1610.06125 [hep-ph]. [Acta Phys. Polon. B 48 (2017) 163].

[214] G. Chen, X.-G. Wu, J.-W. Zhang, H.-Y. Han, H.-B. Fu, Hadronic production of $\Xi_{c c}$ at a fixed-target experiment at the LHC, Phys. Rev. D 89 (7) (2014) 074020, arXiv:1401.6269 [hep-ph].

[215] M. Siddikov, Personal communication.

[216] I. Schmidt, M. Siddikov, Production of pentaquarks in pA-collisions, Phys. Rev. D 93 (9) (2016) 094005, arXiv:1601.05621 [hep-ph].

[217] J. Pumplin, Light-cone models for intrinsic charm and bottom, Phys. Rev. D 73 (2006) 114015, arXiv:hep-ph/0508184 [hep-ph].

[218] J. Pumplin, H.L. Lai, W.K. Tung, The charm parton content of the nucleon, Phys. Rev. D 75 (2007) 054029, arXiv:hep-ph/0701220 [hep-ph].

[219] B.A. Kniehl, G. Kramer, I. Schienbein, H. Spiesberger, Open charm hadroproduction and the charm content of the proton, Phys. Rev. D 79 (2009) 094009, arXiv:0901.4130 [hep-ph].

[220] B.A. Kniehl, G. Kramer, I. Schienbein, H. Spiesberger, Inclusive Charmed-Meson production at the CERN LHC, Eur. Phys. J. C 72 (2012) 2082 , arXiv: 1202.0439 [hep-ph].

[221] V.A. Bednyakov, M.A. Demichev, G.I. Lykasov, T. Stavreva, M. Stockton, Searching for intrinsic charm in the proton at the LHC, Phys. Lett. B 728 (2014) 602-606, arXiv:1305.3548 [hep-ph].

[222] M. Czakon, N.P. Hartland, A. Mitov, E.R. Nocera, J. Rojo, Pinning down the large-x gluon with NNLO top-quark pair differential distributions, J. High Energy Phys. 04 (2017) 044, arXiv: 1611.08609 [hep-ph].

[223] PROSA Collaboration, O. Zenaiev, et al., Impact of heavy-flavour production cross sections measured by the LHCb experiment on parton distribution functions at low x, Eur. Phys. J. C 75 (8) (2015) 396, arXiv: 1503.04581 [hep-ph].

[224] R. Gauld, J. Rojo, Precision determination of the small-x gluon from charm production at LHCb, Phys. Rev. Lett. 118 (7) (2017) 072001 arXiv: 1610.09373 [hep-ph].

[225] J.-P. Lansberg, H.-S. Shao, Double-quarkonium production at a fixed-target experiment at the LHC (AFTER@LHC), Nuclear Phys. B 900 (2015) 273-294, arXiv:1504.06531 [hep-ph].

[226] J.-P. Lansberg, H.-S. Shao, Production of $J / \psi+\eta_{c}$ versus $J / \psi+J / \psi$ at the LHC: Importance of real $\alpha_{s}^{5}$ corrections, Phys. Rev. Lett. 111 (2013) 122001, arXiv:1308.0474 [hep-ph].

[227] J.-P. Lansberg, H.-S. Shao, $J / \psi$-Pair production at large momenta: Indications for double parton scatterings and large $\alpha_{s}^{5}$ contributions, Phys, Lett. B 751 (2015) 479-486, arXiv:1410.8822 [hep-ph].

[228] D. d'Enterria, J. Rojo, Quantitative constraints on the gluon distribution function in the proton from collider isolated-photon data, Nuclear Phys. B 860 (2012) 311-338, arXiv:1202.1762 [hep-ph].

[229] FERMILAB-E706 Collaboration, G. Alverson, et al., Production of direct photons and neutral mesons at large transverse momenta by $\pi^{-}$and $p$ beams at 500-GeV/c, Phys. Rev. D 48 (1993) 5-28.

[230] Fermilab E706 Collaboration, L. Apanasevich, et al., Evidence for parton $k_{T}$ effects in high $p_{T}$ particle production, Phys. Rev. Lett. 81 (1998) 2642-2645, arXiv:hep-ex/9711017 [hep-ex].

[231] Fermilab E706 Collaboration, L. Apanasevich, et al., Measurement of direct photon production at tevatron fixed target energies, Phys. Rev. D 70 (2004) 092009, arXiv:hep-ex/0407011 [hep-ex]

[232] H. Baer, J. Ohnemus, J.F. Owens, A next-to-leading logarithm calculation of direct photon production, Phys. Rev. D 42 (1990) 61-71. 
[233] P. Aurenche, P. Chiappetta, M. Fontannaz, J.P. Guillet, E. Pilon, Next-to-leading order bremsstrahlung contribution to prompt photon production, Nuclear Phys. B 399 (1993) 34-62.

[234] L.E. Gordon, W. Vogelsang, Polarized and unpolarized isolated prompt photon production beyond the leading order, Phys. Rev. D 50 (1994) 1901-1916.

[235] E. Laenen, G. Oderda, G.F. Sterman, Resummation of threshold corrections for single particle inclusive cross-sections, Phys. Lett. B 438 (1998) 173-183, arXiv:hep-ph/9806467 [hep-ph].

[236] S. Catani, M.L. Mangano, P. Nason, Sudakov resummation for prompt photon production in hadron collisions, J. High Energy Phys. 07 (1998) 024, arXiv:hep-ph/9806484 [hep-ph].

[237] N. Kidonakis, J.F. Owens, Effects of higher order threshold corrections in high E(T) jet production, Phys. Rev. D 63 (2001) 054019, arXiv:hep-ph/0007268 [hep-ph].

[238] N. Kidonakis, J.F. Owens, Next-to-next-to-leading order soft gluon corrections in direct photon production, Internat. J. Modern Phys. A 19 (2004) 149-158, arXiv:hep-ph/0307352 [hep-ph].

[239] D. de Florian, W. Vogelsang, Threshold resummation for the prompt-photon cross section revisited, Phys. Rev. D 72 (2005) 014014, arXiv:hep-ph/0506150 [hep-ph].

[240] D. Higinbotham, G.A. Miller, O. Hen, K. Rith, The EMC effect still puzzles after 30 years, CERN Cour. 53N4 (2013) 24, arXiv:1305.7143 [hep-ph]

[241] S. Malace, D. Gaskell, D.W. Higinbotham, I. Cloet, The challenge of the EMC effect: existing data and future directions, Internat. J. Modern Phys. E 23 (08) (2014) 1430013, arXiv:1405.1270 [nucl-ex].

[242] K.J. Eskola, H. Paukkunen, C.A. Salgado, EPS09: A new generation of NLO and LO nuclear parton distribution functions, J. High Energy Phys. 04 (2009) 065, arXiv:0902.4154 [hep-ph].

[243] I. Schienbein, J.Y. Yu, K. Kovarik, C. Keppel, J.G. Morfin, F. Olness, J.F. Owens, PDF nuclear corrections for charged and neutral current processes, Phys. Rev. D 80 (2009) 094004, arXiv:0907.2357 [hep-ph].

[244] A. Kusina, J.-P. Lansberg, I. Schienbein, H.-S. Shao, Gluon shadowing in heavy-flavor production at the LHC, Phys. Rev. Lett. 121 (5) (2018) 052004, arXiv:1712.07024 [hep-ph].

[245] W.T. Giele, S. Keller, Implications of hadron collider observables on parton distribution function uncertainties, Phys. Rev. D 58 (1998) 094023, arXiv:hep-ph/9803393 [hep-ph].

[246] N. Sato, J.F. Owens, H. Prosper, BayesIan reweighting for global fits, Phys. Rev. D 89 (11) (2014) 114020, arXiv:1310.1089 [hep-ph].

[247] NNPDF Collaboration, R.D. Ball, V. Bertone, F. Cerutti, L. Del Debbio, S. Forte, A. Guffanti, J.I. Latorre, J. Rojo, M. Ubiali, Reweighting NNPDFs: the w lepton asymmetry, Nuclear Phys. B 849 (2011) 112-143, arXiv:1012.0836 [hep-ph]; Nuclear Phys. B 855 (2012) 927, (erratum).

[248] A. Kusina, F. Lyonnet, D.B. Clark, E. Godat, T. Jezo, K. Kovarik, F.I. Olness, I. Schienbein, J.Y. Yu, Vector boson production in ppb and pbpb collisions at the LHC and its impact on nCTEQ15 PDFs, Eur. Phys. J. C 77 (7) (2017) 488, arXiv:1610.02925 [nucl-th].

[249] R. Vogt, Gluon shadowing effects on $J / \psi$ and $\Upsilon$ production in $\mathrm{p}+\mathrm{Pb}$ collisions at $\sqrt{S_{N N}}=115 \mathrm{GeV}$ and $\mathrm{p}+\mathrm{Pb}$ collisions at $\sqrt{S_{N N}}=72 \mathrm{GeV}$ at AFTER@LHC, Adv. High Energy Phys. 2015 (2015) 492302, arXiv:1510.03976 [hep-ph].

[250] J.-P. Lansberg, H.-S. Shao, Towards an automated tool to evaluate the impact of the nuclear modification of the gluon density on quarkonium, D and B meson production in proton-nucleus collisions, Eur. Phys. J. C 77 (1) (2017) 1, arXiv:1610.05382 [hep-ph].

[251] H.-S. Shao, HELAC-Onia: An automatic matrix element generator for heavy quarkonium physics, Comput. Phys. Comm. 184 (2013) 2562-2570, arXiv: 1212.5293 [hep-ph].

[252] H.-S. Shao, HELAC-Onia 2.0: an upgraded matrix-element and event generator for heavy quarkonium physics, Comput. Phys. Comm. 198 (2016) 238-259, arXiv:1507.03435 [hep-ph].

[253] F. Arleo, S. Peigne, Quarkonium suppression from coherent energy loss in fixed-target experiments using LHC beams, Adv. High Energy Phys. 2015 (2015) 961951, arXiv:1504.07428 [hep-ph].

[254] AMS Collaboration, M. Aguilar, et al., Precision measurement of the $\left(e^{+}+e^{-}\right)$flux in primary cosmic rays from 0.5 GeV to 1 TeV with the alpha magnetic spectrometer on the international space station, Phys. Rev. Lett. 113 (2014) 221102.

[255] DAMPE Collaboration, G. Ambrosi, et al., Direct detection of a break in the teraelectronvolt cosmic-ray spectrum of electrons and positrons Nature 552 (2017) 63-66, arXiv:1711.10981 [astro-ph.HE].

[256] Fermi-LAT Collaboration, W.B. Atwood, et al., The large area telescope on the Fermi gamma-ray space telescope mission, Astrophys. J. 697 (2009) 1071-1102, arXiv:0902.1089 [astro-ph.IM].

[257] Fermi-LAT Collaboration, M. Ajello, et al., Fermi-LAT Observations of high-energy $\gamma$-ray emission toward the galactic center, Astrophys. J. 819 (1) (2016) 44, arXiv:1511.02938 [astro-ph.HE].

[258] IceCube Collaboration, M.G. Aartsen, et al., Constraints on ultrahigh-energy cosmic-ray sources from a search for neutrinos above 10 PeV with IceCube, Phys. Rev. Lett. 117 (24) (2016) 241101, arXiv:1607.05886 [astro-ph.HE]; Phys. Rev. Lett. 119 (25) (2017) 259902, (erratum).

[259] IceCube Collaboration, M.G. Aartsen, et al., Measurement of the $v_{\mu}$ energy spectrum with ceCube-79, Eur. Phys. J. C 77 (10) (2017) 692, arXiv:1705.07780 [astro-ph.HE].

[260] AMS Collaboration, M. Aguilar, et al., Precision measurement of the proton flux in primary cosmic rays from rigidity 1 GV to 1.8 TV with the alpha magnetic spectrometer on the international space station, Phys. Rev. Lett. 114 (2015) 171103.

[261] AMS Collaboration, M. Aguilar, et al., Antiproton flux, antiproton-to-proton flux ratio, and properties of elementary particle fluxes in primary cosmic rays measured with the alpha magnetic spectrometer on the international space station, Phys. Rev. Lett. 117 (9) (2016) 091103.

[262] AMS Collaboration, M. Aguilar, et al., Precision measurement of the helium flux in primary cosmic rays of rigidities 1.9 GV to 3 TV with the alpha magnetic spectrometer on the international space station, Phys. Rev. Lett. 115 (21) (2015) 211101.

[263] AMS Collaboration, M. Aguilar, et al., Observation of the identical rigidity dependence of He, C, and $\mathrm{O}$ cosmic rays at high rigidities by the alpha magnetic spectrometer on the international space station, Phys. Rev. Lett. 119 (25) (2017) 251101.

[264] AMS Collaboration, M. Aguilar, et al., Observation of new properties of secondary cosmic rays lithium, beryllium, and boron by the alpha magnetic spectrometer on the international space station, Phys. Rev. Lett. 120 (2) (2018) 021101.

[265] R. Enberg, M.H. Reno, I. Sarcevic, Prompt neutrino fluxes from atmospheric charm, Phys. Rev. D 78 (2008) 043005, arXiv:0806.0418 [hep-ph]

[266] A. Bhattacharya, R. Enberg, M.H. Reno, I. Sarcevic, A. Stasto, Perturbative charm production and the prompt atmospheric neutrino flux in light of RHIC and LHC, J. High Energy Phys. 06 (2015) 110, arXiv:1502.01076 [hep-ph].

[267] R. Laha, S.J. Brodsky, Icecube can constrain the intrinsic charm of the proton, Phys. Rev. D 96 (12) (2017) 123002, arXiv:1607.08240 [hep-ph].

[268] F. Riehn, Personal communication.

[269] R.M. Ulrich, C. Baus, R. Engel, A. Fedynitch, U. Kraemer, T. Pierog, F. Riehn, The impact of a fixed-target experiment with LHC beam for astroparticle physics, PoS ICRC 2015 (2016) 407.

[270] A.V. Giannini, V.P. Goncalves, F.S. Navarra, Intrinsic charm contribution to the prompt atmospheric neutrino flux, Phys. Rev. D 98 (1) (2018) 014012, arXiv:1803.01728 [hep-ph].

[271] I.A. Grenier, J.H. Black, A.W. Strong, The nine lives of cosmic rays in galaxies, Ann. Rev. Astron. Astrophys. 53 (2015) $199-246$.

[272] P. Blasi, The origin of galactic cosmic rays, Astron. Astrophys. Rev. 21 (2013) 70, arXiv:1311.7346 [astro-ph.HE].

[273] A.W. Strong, I.V. Moskalenko, V.S. Ptuskin, Cosmic-ray propagation and interactions in the Galaxy, Ann. Rev. Nucl. Part. Sci. 57 (2007) 285-327, arXiv:astro-ph/0701517 [astro-ph]. 
[274] I.V. Moskalenko, A.W. Strong, Production and propagation of cosmic ray positrons and electrons, Astrophys. J. 493 (1998) 694-707, arXiv: astro-ph/9710124 [astro-ph].

[275] A.E. Vladimirov, S.W. Digel, G. Johannesson, P.F. Michelson, I.V. Moskalenko, P.L. Nolan, E. Orlando, T.A. Porter, A.W. Strong, GALPROP WebRun: an internet-based service for calculating galactic cosmic ray propagation and associated photon emissions, Comput. Phys. Comm. 182 (2011) 1156-1161, arXiv:1008.3642 [astro-ph.HE].

[276] E. Orlando, G. Johannesson, I.V. Moskalenko, T.A. Porter, A. Strong, GALPROP cosmic-ray propagation code: recent results and updates, Nucl. Part. Phys. Proc. 297-299 (2018) 129-134, arXiv:1712.09755 [astro-ph.HE].

[277] M. Korsmeier, F. Donato, M. Di Mauro, Production cross sections of cosmic antiprotons in the light of new data from NA61 and LHCb experiments, Phys. Rev. D 97 (2018) 103019, arXiv:1802.03030 [astro-ph.HE].

[278] NA61/SHINE Collaboration, A. Aduszkiewicz, et al., Measurements of $\pi^{ \pm}, \mathrm{K}^{ \pm}, \mathrm{p}$ and $\overline{\mathrm{p}}$ spectra in proton-proton interactions at 20, 31, 40, 80 and $158 \mathrm{GeV} / \mathrm{c}$ with the NA61/SHINE spectrometer at the CERN SPS, Eur. Phys. J. C 77 (10) (2017) 671, http://dx.doi.org/10.1140/epjc/s10052017-5260-4, arXiv:1705.02467.

[279] M. di Mauro, F. Donato, A. Goudelis, P.D. Serpico, New evaluation of the antiproton production cross section for cosmic ray studies, Phys. Rev. D 90 (8) (2014) 085017, arXiv:1408.0288 [hep-ph].

[280] R. Kappl, M.W. Winkler, The cosmic ray antiproton background for AMS-02, J. Cosmol. Astropart. Phys. 1409 (2014) 051, arXiv:1408.0299 [hep-ph].

[281] F. Donato, D. Maurin, P. Salati, A. Barrau, G. Boudoul, R. Taillet, Anti-protons from spallations of cosmic rays on interstellar matter, Astrophys J. 563 (2001) 172-184, arXiv:astro-ph/0103150 [astro-ph].

[282] M. Jebramcik, J. Jowett, Prospects for future asymmetric collisions in the LHC, in: Proceedings, 10th International Particle Accelerator Conference (IPAC2019): Melbourne, Australia, (2019) May 19-24, p. MOPMP024.

[283] A. Bacchetta, D. Boer, M. Diehl, P.J. Mulders, Matches and mismatches in the descriptions of semi-inclusive processes at low and high transverse momentum, J. High Energy Phys. 08 (2008) 023, arXiv:0803.0227 [hep-ph].

[284] A. Bacchetta, G. Bozzi, M.G. Echevarria, C. Pisano, A. Prokudin, M. Radici, Azimuthal asymmetries in unpolarized SIDIS and Drell-Yan processes: a case study towards TMD factorization at subleading twist, Phys. Lett. B 797 (2019) 134850, arXiv:1906.07037 [hep-ph].

[285] M. Anselmino, M. Boglione, U. D’Alesio, S. Melis, F. Murgia, A. Prokudin, Sivers effect in drell-yan processes, Phys. Rev. D 79 (2009) 054010 http://dx.doi.org/10.1103/PhysRevD.79.054010, arXiv:0901.3078.

[286] B.E. Bonner, et al., Analyzing power measurement in inclusive pi0 production at high x(f), Phys. Rev. Lett. 61 (1988) 1918.

[287] E704, E581 Collaboration, D.L. Adams, et al., Comparison of spin asymmetries and cross-sections in $\pi^{0}$ production by $200 \mathrm{GeV}$ polarized anti-protons and protons, Phys. Lett. B 261 (1991) 201-206.

[288] M.G. Echevarria, A. Idilbi, A. Schafer, I. Scimemi, Model-independent evolution of transverse momentum dependent distribution functions (TMDs) at NNLL, Eur. Phys. J. C 73 (12) (2013) 2636, arXiv:1208.1281 [hep-ph].

[289] T.C. Rogers, An overview of transverse-momentum-dependent factorization and evolution, Eur. Phys. J. A 52 (6) (2016) 153, arXiv:1509.04766 [hep-ph].

[290] J. Collins, T.C. Rogers, Connecting different TMD factorization formalisms in QCD, Phys. Rev. D 96 (5) (2017) 054011, arXiv: 1705.07167 [hep-ph]

[291] A. Bacchetta, F. Delcarro, C. Pisano, M. Radici, A. Signori, Extraction of partonic transverse momentum distributions from semi-inclusive deep-inelastic scattering, Drell-Yan and Z-boson production, J. High Energy Phys. 06 (2017) 081, arXiv:1703.10157 [hep-ph].

[292] I. Scimemi, A. Vladimirov, Analysis of vector boson production within TMD factorization, Eur. Phys. J. C 78 (2) (2018) 89 , arXiv:1706.01473 [hep-ph].

[293] I. Scimemi, A. Vladimirov, Systematic analysis of double-scale evolution, J. High Energy Phys. 08 (2018) 003, arXiv:1803.11089 [hep-ph].

[294] M.G. Echevarria, A. Idilbi, Z.-B. Kang, I. Vitev, QCD evolution of the Sivers asymmetry, Phys. Rev. D 89 (2014) 074013, arXiv:1401.5078 [hep-ph].

[295] P. Sun, F. Yuan, Energy evolution for the sivers asymmetries in hard processes, Phys. Rev. D 88 (2013) 034016, arXiv:1304.5037 [hep-ph].

[296] L.D. Isenhower, et al., Polarized Drell-Yan measurements with the Fermilab Main Injector, FERMILAB-PROPOSAL-1027.

[297] T. Liu, B.-Q. Ma, Azimuthal asymmetries in lepton-pair production at a fixed-target experiment using the LHC beams (AFTER), Eur. Phys. J. C 72 (2012) 2037, arXiv:1203.5579 [hep-ph].

[298] M. Anselmino, U. D’Alesio, S. Melis, Transverse single-spin asymmetries in proton-proton collisions at the AFTER@LHC experiment in a TMD factorisation scheme, Adv. High Energy Phys. 2015 (2015) 475040, arXiv:1504.03791 [hep-ph].

[299] E. Steffens, Estimation of the performance of a HERMES-type gas target internal to the LHC, PoS PSTP 2015 (2015) 019.

[300] COMPASS Collaboration, F. Gautheron, et al., COMPASS-II Proposal, 2010, SPSC-P-340, CERN-SPSC-2010-014.

[301] RHIC Spin Collaboration, Transverse-spin Drell-Yan physics at RHIC, 2007, spin.riken.bnl.gov/rsc/write-up/dy_final.pdf.

[302] C. Brown, et al., Letter of intent for a Drell-Yan experiment with a polarized proton target, 2014, FERMILAB-PROPOSAL-1039.

[303] NICA Collaboration. nica.jinr.ru.

[304] sPHENIX Collaboration, sPHENIX Forward Instrumentation : A Letter of Intent, 2017. indico.bnl.gov/event/3867/attachments/10442/12745/sPHcQCD-2017-001_draft_2017_06_02.pdf.

[305] PANDA Collaboration. panda.gsi.de/.

[306] D. Kikola, M.G. Echevarria, C. Hadjidakis, J.-P. Lansberg, C. Lorce, L. Massacrier, C.M. Quintans, A. Signori, B. Trzeciak, Feasibility studies for single transverse-spin asymmetry measurements at a fixed-target experiment using the LHC proton and lead beams (AFTER@LHC), Few Body Syst. 58 (4) (2017) 139, arXiv:1702.01546 [hep-ex].

[307] Jefferson Lab Hall A Collaboration, X. Qian, et al., Single spin asymmetries in charged pion production from semi-inclusive deep inelastic scattering on a transversely polarized ${ }^{3} \mathrm{He}$ target, Phys. Rev. Lett. 107 (2011) 072003, arXiv:1106.0363 [nucl-ex].

[308] Jefferson Lab Hall A Collaboration, J. Huang, et al., Beam-target double spin asymmetry $A_{L T}$ in charged pion production from deep inelastic scattering on a transversely polarized He-3 target at $1.4<Q^{2}<2.7 \mathrm{GeV}^{2}$, Phys. Rev. Lett. 108 (2012) 052001, arXiv:1108.0489 [nucl-ex].

[309] A. Signori, A. Bacchetta, M. Radici, G. Schnell, Investigations into the flavor dependence of partonic transverse momentum, J. High Energy Phys. 11 (2013) 194, arXiv:1309.3507 [hep-ph].

[310] M. Anselmino, M. Boglione, J.O. Gonzalez Hernandez, S. Melis, A. Prokudin, Unpolarised transverse momentum dependent distribution and fragmentation functions from SIDIS multiplicities, J. High Energy Phys. 04 (2014) 005, arXiv:1312.6261 [hep-ph].

[311] U. D’Alesio, M.G. Echevarria, S. Melis, I. Scimemi, Non-perturbative QCD effects in $q_{T}$ spectra of Drell-Yan and Z-boson production, J. High Energy Phys. 11 (2014) 098, arXiv:1407.3311 [hep-ph].

[312] HERMES Collaboration, A. Airapetian, et al., Transverse target single-spin asymmetry in inclusive electroproduction of charged pions and kaons, Phys. Lett. B 728 (2014) 183-190, arXiv:1310.5070 [hep-ex].

[313] COMPASS Collaboration, C. Adolph, et al., II - EXperimental investigation of transverse spin asymmetries in $\mu-p$ SIDIS processes: Sivers asymmetries, Phys. Lett. B 717 (2012) 383-389, arXiv:1205.5122 [hep-ex].

[314] COMPASS Collaboration, E.S. Ageev, et al., A new measurement of the Collins and Sivers asymmetries on a transversely polarised deuteron target, Nuclear Phys. B 765 (2007) 31-70, arXiv:hep-ex/0610068 [hep-ex].

[315] K. Kanazawa, Y. Koike, A. Metz, D. Pitonyak, Transverse single-spin asymmetries in proton-proton collisions at the AFTER@LHC experiment, Adv. High Energy Phys. 2015 (2015) 257934, arXiv:1502.04021 [hep-ph]. 
[316] L. Gamberg, Z.-B. Kang, Single transverse spin asymmetry of prompt photon production, Phys. Lett. B 718 (2012) 181-188, arXiv:1208.1962 [hep-ph].

[317] U. D’Alesio, F. Murgia, Azimuthal and single spin asymmetries in hard scattering processes, Prog. Part. Nucl. Phys. 61 (2008) 394-454, arXiv:0712.4328 [hep-ph].

[318] COMPASS Collaboration, K. Kurek, A. Szabelski, The gluon contribution to the sivers effect COMPASS results, J. Phys. Conf. Ser. 678 (1) (2016) 012055.

[319] C. Pisano, D. Boer, S.J. Brodsky, M.G.A. Buffing, P.J. Mulders, Linear polarization of gluons and photons in unpolarized collider experiments, J. High Energy Phys. 10 (2013) 024, arXiv:1307.3417 [hep-ph].

[320] R.M. Godbole, A. Kaushik, A. Misra, Transverse single spin asymmetry in $p+p^{\uparrow} \rightarrow D+X$, Phys. Rev. D 94 (11) (2016) 114022, arXiv:1606.01818 [hep-ph].

[321] U. D’Alesio, F. Murgia, C. Pisano, P. Taels, Probing the gluon sivers function in $p^{\uparrow} p \rightarrow J / \psi X$ and $p^{\uparrow} p \rightarrow D X$, Phys. Rev. D 96 (3) (2017) 036011, arXiv:1705.04169 [hep-ph].

[322] Z.-B. Kang, J.-W. Qiu, W. Vogelsang, F. Yuan, Accessing tri-gluon correlations in the nucleon via the single spin asymmetry in open charm production, Phys. Rev. D 78 (2008) 114013, arXiv:0810.3333 [hep-ph].

[323] X.-D. Ji, Gluon correlations in the transversely polarized nucleon, Phys. Lett. B 289 (1992) 137-142.

[324] H. Beppu, Y. Koike, K. Tanaka, S. Yoshida, Contribution of twist-3 multi-gluon correlation functions to single spin asymmetry in semi-inclusive deep inelastic scattering, Phys. Rev. D 82 (2010) 054005, arXiv:1007.2034 [hep-ph].

[325] M. Cacciari, M. Greco, P. Nason, The P(T) spectrum in heavy flavor hadroproduction, J. High Energy Phys. 05 (1998) 007, arXiv:hep-ph/9803400 [hep-ph].

[326] M. Cacciari, S. Frixione, P. Nason, The p(T) spectrum in heavy flavor photoproduction, J. High Energy Phys. 03 (2001) 006, arXiv:hep-ph/0102134 [hep-ph].

[327] M. Anselmino, M. Boglione, U. D’Alesio, E. Leader, F. Murgia, Accessing sivers gluon distribution via transverse single spin asymmetries in $p^{\uparrow} p \rightarrow D X$ processes at RHIC, Phys. Rev. D 70 (2004) 074025, arXiv:hep-ph/0407100 [hep-ph].

[328] U. D'Alesio, F. Murgia, C. Pisano, Towards a first estimate of the gluon sivers function from $A_{N}$ data in pp collisions at RHIC, J. High Energy Phys. 09 (2015) 119, arXiv:1506.03078 [hep-ph].

[329] PHENIX Collaboration, C. Aidala, et al., Cross section and transverse single-spin asymmetry of muons from open heavy-flavor decays in polarized $p+p$ collisions at $\sqrt{s}=200 \mathrm{GeV}$, Phys. Rev. D 95 (11) (2017) 112001, arXiv:1703.09333 [hep-ex].

[330] D. Kikola, Prospects for open heavy flavor measurements in heavy ion and p + A collisions in a fixed-target experiment at the LHC, Adv. High Energy Phys. 2015 (2015) 783134.

[331] L. Massacrier, B. Trzeciak, F. Fleuret, C. Hadjidakis, D. Kikola, J.P. Lansberg, H.S. Shao, Feasibility studies for quarkonium production at a fixed-target experiment using the LHC proton and lead beams (AFTER@LHC), Adv. High Energy Phys. 2015 (2015) 986348, arXiv:1504.05145 [hep-ex].

[332] F. Yuan, Heavy quarkonium production in single transverse polarized high energy scattering, Phys. Rev. D 78 (2008) 014024, arXiv:0801.4357 [hep-ph].

[333] ALICE Collaboration, J. Adam, et al., Inclusive quarkonium production at forward rapidity in pp collisions at $\sqrt{s}=8 \mathrm{TeV}$, Eur. Phys. J. C 76 (4) (2016) 184, arXiv:1509.08258 [hep-ex].

[334] ALICE Collaboration, B.B. Abelev, et al., Measurement of quarkonium production at forward rapidity in $p p$ collisions at $\sqrt{s}=7$ TeV, Eur. Phys. J. C 74 (8) (2014) 2974, arXiv:1403.3648 [nucl-ex].

[335] D. Boer, W.J. den Dunnen, TMD evolution and the higgs transverse momentum distribution, Nuclear Phys. B 886 (2014) 421-435, arXiv: 1404.6753 [hep-ph].

[336] M.G. Echevarria, T. Kasemets, P.J. Mulders, C. Pisano, QCD evolution of (un)polarized gluon TMDPDFs and the Higgs $q_{T}$-distribution, J. High Energy Phys. 07 (2015) 158, arXiv:1502.05354 [hep-ph]; J. High Energy Phys. 05 (2017) 073, (erratum).

[337] LHCb Collaboration, R. Aaij, et al., Measurement of the ratio of prompt $\chi_{c}$ to $J / \psi$ production in $p p$ collisions at $\sqrt{s}=7 \mathrm{TeV}$, Phys. Lett. B 718 (2012) 431-440, arXiv:1204.1462 [hep-ex].

[338] LHCb Collaboration, R. Aaij, et al., Measurement of the cross-section ratio $\sigma\left(\chi_{c 2}\right) / \sigma\left(\chi_{c 1}\right)$ for prompt $\chi_{c}$ production at $\sqrt{s}=7$ TeV, Phys. Lett. B 714 (2012) 215-223, arXiv:1202.1080 [hep-ex].

[339] LHCb Collaboration, R. Aaij, et al., Measurement of the $\eta_{c}(1 S)$ production cross-section in proton-proton collisions via the decay $\eta_{c}(1 S) \rightarrow p \bar{p}$, Eur. Phys. J. C 75 (7) (2015) 311, arXiv:1409.3612 [hep-ex].

[340] LHCb Collaboration, R. Aaij, et al., Observation of $\eta_{c}(2 S) \rightarrow p \bar{p}$ and search for $X(3872) \rightarrow p \bar{p}$ decays, Phys. Lett. B 769 (2017) 305-313, arXiv: 1607.06446 [hep-ex].

[341] J.-P. Lansberg, H.-S. Shao, H.-F. Zhang, $\eta_{c}^{\prime}$ hadroproduction at next-to-leading order and its relevance to $\psi^{\prime}$ production, Phys. Lett. B 786 (2018) 342-346, arXiv:1711.00265 [hep-ph].

[342] A. Schafer, J. Zhou, Transverse single spin asymmetry in hadronic $\eta_{c, b}$ production, Phys. Rev. D 88 (1) (2013) 014008 , arXiv:1302.4600 [hep-ph].

[343] J.-W. Qiu, M. Schlegel, W. Vogelsang, Probing gluonic spin-orbit correlations in photon pair production, Phys. Rev. Lett. 107 (2011) 062001 , arXiv:1103.3861 [hep-ph].

[344] D. Boer, C. Pisano, Impact of gluon polarization on higgs boson plus jet production at the LHC, Phys. Rev. D 91 (7) (2015) 074024, arXiv: 1412.5556 [hep-ph].

[345] J.P. Lansberg, Back-to-back isolated photon-quarkonium production at the LHC and the transverse-momentum-dependent distributions of the gluons in the proton, Int. J. Mod. Phys. Conf. Ser. 40 (2016) 1660015, arXiv:1502.02263 [hep-ph].

[346] A. Signori, Flavor and Evolution Effects in TMD Phenomenology, (Ph.D. thesis), Vrije U., Amsterdam, 2016, https://userweb.jlab.org/ asignori/ research/PhD_thesis_Andrea.pdf.

[347] D. Boer, Gluon TMDs in quarkonium production, Few Body Syst. 58 (2) (2017) 32, arXiv:1611.06089 [hep-ph].

[348] F. Scarpa, D. Boer, M.G. Echevarria, J.-P. Lansberg, C. Pisano, M. Schlegel, Studies of gluon TMDs and their evolution using quarkonium-pair production at the LHC. arXiv:1909.05769 [hep-ph].

[349] S. Arnold, A. Metz, M. Schlegel, Dilepton production from polarized hadron hadron collisions, Phys. Rev. D 79 (2009) 034005, arXiv:0809.2262 [hep-ph].

[350] V. Barone, S. Melis, A. Prokudin, The Boer-Mulders effect in unpolarized SIDIS: An analysis of the COMPASS and HERMES data on the cos $2 \phi$ asymmetry, Phys. Rev. D 81 (2010) 114026, arXiv:0912.5194 [hep-ph].

[351] J.-P. Lansberg, C. Pisano, F. Scarpa, M. Schlegel, Pinning down the linearly-polarised gluons inside unpolarised protons using quarkonium-pair production at the LHC, Phys. Lett. B 784 (2018) 217-222, arXiv:1710.01684 [hep-ph].

[352] S. Meissner, A. Metz, K. Goeke, Relations between generalized and transverse momentum dependent parton distributions, Phys. Rev. D 76 (2007) 034002, arXiv:hep-ph/0703176 [hep-ph].

[353] A. Metz, J. Zhou, Distribution of linearly polarized gluons inside a large nucleus, Phys. Rev. D 84 (2011) 051503, arXiv:1105.1991 [hep-ph].

[354] F. Dominguez, J.-W. Qiu, B.-W. Xiao, F. Yuan, On the linearly polarized gluon distributions in the color dipole model, Phys. Rev. D 85 (2012) 045003, arXiv:1109.6293 [hep-ph]. 
[355] M.G. Echevarria, Proper TMD factorization for quarkonia production: $p p \rightarrow \eta_{c, b}$ as a study case, J. High Energy Phys. 10 (2019) 144, arXiv:1907.06494 [hep-ph].

[356] S. Fleming, Y. Makris, T. Mehen, An effective field theory approach to quarkonium at small transverse momentum, arXiv:1910.03586 [hep-ph]

[357] D. Boer, S.J. Brodsky, P.J. Mulders, C. Pisano, Direct probes of linearly polarized gluons inside unpolarized hadrons, Phys. Rev. Lett. 106 (2011) 132001, arXiv: 1011.4225 [hep-ph].

[358] S. Catani, M. Grazzini, QCD transverse-momentum resummation in gluon fusion processes, Nuclear Phys. B 845 (2011) 297-323, arXiv: 1011.3918 [hep-ph].

[359] D. Boer, W.J. den Dunnen, C. Pisano, M. Schlegel, W. Vogelsang, Linearly polarized gluons and the higgs transverse momentum distribution, Phys. Rev. Lett. 108 (2012) 032002, arXiv:1109.1444 [hep-ph].

[360] D. Boer, W.J. den Dunnen, C. Pisano, M. Schlegel, Determining the Higgs spin and parity in the diphoton decay channel, Phys. Rev. Lett. 111 (3) (2013) 032002, arXiv:1304.2654 [hep-ph].

[361] P.M. Nadolsky, C. Balazs, E.L. Berger, C.P. Yuan, Gluon-gluon contributions to the production of continuum diphoton pairs at hadron colliders, Phys. Rev. D 76 (2007) 013008, arXiv:hep-ph/0702003 [hep-ph].

[362] A. Mukherjee, S. Rajesh, Probing transverse momentum dependent parton distributions in charmonium and bottomonium production, Phys. Rev. D 93 (5) (2016) 054018, arXiv:1511.04319 [hep-ph].

[363] A. Mukherjee, S. Rajesh, Linearly polarized gluons in charmonium and bottomonium production in color octet model, Phys. Rev. D 95 (3) (2017) 034039, arXiv:1611.05974 [hep-ph].

[364] B. Gong, J.-P. Lansberg, C. Lorce, J. Wang, Next-to-leading-order QCD corrections to the yields and polarisations of J/Psi and upsilon directly produced in association with a Z boson at the LHC, J. High Energy Phys. 03 (2013) 115, arXiv:1210.2430 [hep-ph].

[365] J.P. Ma, J.X. Wang, S. Zhao, Transverse momentum dependent factorization for quarkonium production at low transverse momentum, Phys. Rev. D 88 (1) (2013) 014027, arXiv:1211.7144 [hep-ph].

[366] J.P. Ma, J.X. Wang, S. Zhao, Breakdown of QCD factorization for P-wave quarkonium production at low transverse momentum, Phys. Lett. B 737 (2014) 103-108, arXiv:1405.3373 [hep-ph].

[367] M. Boglione, A. Prokudin, Phenomenology of transverse spin: past, present and future, Eur. Phys. J. A 52 (6) (2016) 154, arXiv:1511.06924 [hep-ph].

[368] B.-Q. Ma, I. Schmidt, The quark orbital angular momentum in a light cone representation, Phys. Rev. D 58 (1998) 096008, arXiv:hep-ph/9808202 [hep-ph].

[369] J. She, J. Zhu, B.-Q. Ma, Pretzelosity $h_{1 T}^{\perp}$ and quark orbital angular momentum, Phys. Rev. D 79 (2009) 054008, arXiv:0902.3718 [hep-ph].

[370] H. Avakian, A.V. Efremov, P. Schweitzer, F. Yuan, The transverse momentum dependent distribution functions in the bag model, Phys. Rev. D 81 (2010) 074035, arXiv:1001.5467 [hep-ph].

[371] A.V. Efremov, P. Schweitzer, O.V. Teryaev, P. Zavada, Images of quark intrinsic motion in covariant parton model, PoS DIS 2010 (2010) 253 arXiv: 1008.3827 [hep-ph].

[372] C. Lorce, B. Pasquini, M. Vanderhaeghen, Unified framework for generalized and transverse-momentum dependent parton distributions within a $3 Q$ light-cone picture of the nucleon, J. High Energy Phys. 05 (2011) 041, arXiv:1102.4704 [hep-ph].

[373] C. Lorce, B. Pasquini, Pretzelosity TMD and quark orbital angular momentum, Phys. Lett. B 710 (2012) 486-488, arXiv:1111.6069 [hep-ph].

[374] C. Lorce, B. Pasquini, On the origin of model relations among transverse-momentum dependent parton distributions, Phys. Rev. D 84 (2011) 034039, arXiv:1104.5651 [hep-ph].

[375] M. Burkardt, H. B.C., Angular momentum decomposition for an electron, Phys. Rev. D 79 (2009) 071501, arXiv:0812.1605 [hep-ph].

[376] C. Lorce, B. Pasquini, Quark wigner distributions and orbital angular momentum, Phys. Rev. D 84 (2011) 014015, arXiv:1106.0139 [hep-ph].

[377] M. Burkardt, Impact parameter dependent parton distributions and transverse single spin asymmetries, Phys. Rev. D 66 (2002) 114005, arXiv:hep-ph/0209179 [hep-ph].

[378] M. Burkardt, Chromodynamic lensing and transverse single spin asymmetries, Nuclear Phys. A 735 (2004) 185-199, arXiv:hep-ph/0302144 [hep-ph].

[379] M. Burkardt, D.S. Hwang, Sivers asymmetry and generalized parton distributions in impact parameter space, Phys. Rev. D 69 (2004) 074032 arXiv:hep-ph/0309072 [hep-ph].

[380] L. Gamberg, M. Schlegel, Final state interactions and the transverse structure of the pion using non-perturbative eikonal methods, Phys. Lett. B 685 (2010) 95-103, arXiv:0911.1964 [hep-ph].

[381] A. Bacchetta, M. Radici, Constraining quark angular momentum through semi-inclusive measurements, Phys. Rev. Lett. 107 (2011) 212001 , arXiv:1107.5755 [hep-ph].

[382] M. Guidal, M.V. Polyakov, A.V. Radyushkin, M. Vanderhaeghen, Nucleon form-factors from generalized parton distributions, Phys. Rev. D 72 (2005) 054013, arXiv:hep-ph/0410251 [hep-ph].

[383] M. Diehl, T. Feldmann, R. Jakob, P. Kroll, Generalized parton distributions from nucleon form-factor data, Eur. Phys. J. C 39 (2005) 1-39, arXiv:hep-ph/0408173 [hep-ph].

[384] S. Ahmad, H. Honkanen, S. Liuti, S.K. Taneja, Generalized parton distributions from hadronic observables: Zero skewness, Phys. Rev. D 75 (2007) 094003, arXiv:hep-ph/0611046 [hep-ph].

[385] S.V. Goloskokov, P. Kroll, The target asymmetry in hard vector-meson electroproduction and parton angular momenta, Eur. Phys. J. C 59 (2009) 809-819, arXiv:0809.4126 [hep-ph].

[386] M. Diehl, P. Kroll, Nucleon form factors, generalized parton distributions and quark angular momentum, Eur. Phys. J. C 73 (4) (2013) 2397 arXiv:1302.4604 [hep-ph].

[387] C. Lorce, B. Pasquini, Multipole decomposition of the nucleon transverse phase space, Phys. Rev. D 93 (3) (2016) 034040, arXiv:1512.06744 [hep-ph].

[388] A.J. Baltz, The physics of ultraperipheral collisions at the LHC, Phys. Rep. 458 (2008) 1-171, arXiv:0706.3356 [nucl-ex].

[389] J.P. Lansberg, L. Szymanowski, J. Wagner, Lepton-pair production in ultraperipheral collisions at AFTER@LHC, J. High Energy Phys. 09 (2015) 087, arXiv:1504.02733 [hep-ph].

[390] M. Diehl, Generalized parton distributions, Phys. Rep. 388 (2003) 41-277, arXiv:hep-ph/0307382 [hep-ph].

[391] A.V. Belitsky, A.V. Radyushkin, Unraveling hadron structure with generalized parton distributions, Phys. Rep. 418 (2005) 1-387, arXiv:hep$\mathrm{ph} / 0504030$ [hep-ph].

[392] M. Burkardt, Impact parameter dependent parton distributions and off forward parton distributions for $\zeta \rightarrow 0$, Phys. Rev. D 62 (2000) 071503 , arXiv:hep-ph/0005108 [hep-ph]; Phys. Rev. D 66 (2002) 119903, (erratum).

[393] E.R. Berger, M. Diehl, B. Pire, Time - like compton scattering: Exclusive photoproduction of lepton pairs, Eur. Phys. J. C 23 (2002) 675-689, arXiv:hep-ph/0110062 [hep-ph].

[394] V.P. Goncalves, W.K. Sauter, $\eta_{c}$ production in photon-induced interactions at a fixed target experiment at LHC as a probe of the odderon, Phys. Rev. D 91 (9) (2015) 094014, arXiv:1503.05112 [hep-ph].

[395] S.R. Klein, J. Nystrand, J. Seger, Y. Gorbunov, J. Butterworth, STARlight: A Monte Carlo simulation program for ultra-peripheral collisions of relativistic ions, Comput. Phys. Comm. 212 (2017) 258-268, arXiv:1607.03838 [hep-ph]. 
[396] J.P. Lansberg, L. Massacrier, L. Szymanowski, J. Wagner, Single-transverse-spin asymmetries in exclusive photo-production of $J / \psi$ in ultraperipheral collisions in the fixed-target mode at the LHC and in the collider mode at RHIC, Phys. Lett. B 793 (2019) 33-40, arXiv:1812.04553 [hep-ph].

[397] D.Yu. Ivanov, A. Schafer, L. Szymanowski, G. Krasnikov, Exclusive photoproduction of a heavy vector meson in QCD, Eur. Phys. J. C 34 (3) (2004) 297-316, arXiv:hep-ph/0401131 [hep-ph]; Eur. Phys. J. C 75 (2) (2015) 75, (erratum).

[398] L. Massacrier, J.P. Lansberg, L. Szymanowski, J. Wagner, Quarkonium-photoproduction prospects at a fixed-target experiment at the LHC (AFTER@LHC), in: Photon 2017: International Conference on the Structure and the Interactions of the Photon and 22th International Workshop on Photon-Photon Collisions and the International Workshop on High Energy Photon Colliders CERN, Geneva, Switzerland, 2017 May 22-26, 2017, arXiv:1709.09044 [nucl-ex]. http://inspirehep.net/record/1625748/files/arXiv:1709.09044.pdf.

[399] J. Koempel, P. Kroll, A. Metz, J. Zhou, Exclusive production of quarkonia as a probe of the GPD E for gluons, Phys. Rev. D 85 (2012) 051502, arXiv:1112.1334 [hep-ph].

[400] V.P. Goncalves, Investigating the transverse single spin asymmetry in the inelastic $J / \Psi$ photoproduction in $p^{\uparrow} p$ and $p^{\uparrow} A$ collisions, Phys. Rev. D 97 (1) (2018) 014001, arXiv:1710.01674 [hep-ph].

[401] V.P. Goncalves, M.M. Jaime, Exclusive vector meson photoproduction in fixed - target collisions at the LHC, Eur. Phys. J. C 78 (9) (2018) 693, arXiv: 1802.04713 [hep-ph].

[402] NNPDF Collaboration, E.R. Nocera, R.D. Ball, S. Forte, G. Ridolfi, J. Rojo, A first unbiased global determination of polarized PDFs and their uncertainties, Nuclear Phys. B 887 (2014) 276-308, arXiv:1406.5539 [hep-ph].

[403] T. Melson, H.-T. Janka, R. Bollig, F. Hanke, A. Marek, B. Müller, Neutrino-driven explosion of a 20 solar-mass star in three dimensions enabled by strange-quark contributions to neutrino-nucleon scattering, Astrophys. J. 808 (2) (2015) L42, arXiv:1504.07631 [astro-ph.SR].

[404] T.J. Hobbs, M. Alberg, G.A. Miller, Role of nucleon strangeness in supernova explosions, Phys. Rev. C 93 (5) (2016) 052801, arXiv:1601.01729 [astro-ph.HE].

[405] QCDSF Collaboration, G.S. Bali, et al., Strangeness contribution to the proton spin from lattice QCD, Phys. Rev. Lett. 108 (2012) 222001, arXiv:1112.3354 [hep-lat].

[406] C. Alexandrou, M. Constantinou, K. Hadjiyiannakou, K. Jansen, C. Kallidonis, G. Koutsou, A. Vaquero Avilés-Casco, C. Wiese, Nucleon spin and momentum decomposition using lattice QCD simulations, Phys. Rev. Lett. 119 (14) (2017) 142002, arXiv:1706.02973 [hep-lat].

[407] JLQCD Collaboration, N. Yamanaka, S. Hashimoto, T. Kaneko, H. Ohki, Nucleon charges with dynamical overlap fermions, Phys. Rev. D 98 (5) (2018) 054516, arXiv:1805.10507 [hep-lat].

[408] J. Liang, Y.-B. Yang, T. Draper, M. Gong, K.-F. Liu, Quark spins and anomalous ward identity, Phys. Rev. D 98 (7) (2018) 074505 , arXiv:1806.08366 [hep-ph].

[409] H.-W. Lin, R. Gupta, B. Yoon, Y.-C. Jang, T. Bhattacharya, Quark contribution to the proton spin from 2+1+1-flavor lattice QCD, Phys. Rev. D 98 (9) (2018) 094512, arXiv:1806.10604 [hep-lat].

[410] HERMES Collaboration, A. Airapetian, et al., Measurement of parton distributions of strange quarks in the nucleon from charged-Kaon production in deep-inelastic scattering on the deuteron, Phys. Lett. B 666 (2008) 446-450, arXiv:0803.2993 [hep-ex].

[411] STAR Collaboration, B.I. Abelev, et al., Longitudinal spin transfer to lambda and anti-lambda hyperons in polarized proton-proton collisions at $\sqrt{s}=200 \mathrm{GeV}$, Phys. Rev. D 80 (2009) 111102, arXiv:0910.1428 [hep-ex].

[412] COMPASS Collaboration, M. Alekseev, et al., Measurement of the longitudinal spin transfer to lambda and anti-lambda hyperons in polarised muon DIS, Eur. Phys. J. C 64 (2009) 171-179, arXiv:0907.0388 [hep-ex].

[413] NOMAD Collaboration, P. Astier, et al., Measurement of the anti-lambda polarization in muon-neutrino charged current interactions in the nomad experiment, Nucl. Phys. B 605 (2001) 3-14, http://dx.doi.org/10.1016/S0550-3213(01)00181-X, arXiv:hep-ex/0103047.

[414] HERMES Collaboration, A. Airapetian, et al., Longitudinal spin transfer to the lambda hyperon in semi-inclusive deep-inelastic scattering, Phys. Rev. D 74 (2006) 072004, http://dx.doi.org/10.1103/PhysRevD.74.072004, arXiv:hep-ex/0607004.

[415] M. Burkardt, R.L. Jaffe, Polarized q $\rightarrow>$ lambda fragmentation functions from e+ e- -> lambda + x, Phys. Rev. Lett. 70 (1993) 2537-2540 http://dx.doi.org/10.1103/PhysRevLett.70.2537, arXiv:hep-ph/9302232.

[416] Particle Data Group Collaboration, P.A. Zyla, et al., Review of particle physics, PTEP 2020 (8) (2020) 083C01, http://dx.doi.org/10.1093/ptep/ ptaa 104.

[417] ALICE Collaboration, K. Aamodt, et al., Strange particle production in proton-proton collisions at sqrt(s) = 0.9 TeV with ALICE at the LHC, Eur. Phys. J. C 71 (2011) 1594, arXiv:1012.3257 [hep-ex].

[418] J. Engel, M.J. Ramsey-Musolf, U. van Kolck, Electric dipole moments of nucleons, nuclei, and atoms: The standard model and beyond, Prog. Part. Nucl. Phys. 71 (2013) 21-74, arXiv:1303.2371 [nucl-th].

[419] N. Yamanaka, B.K. Sahoo, N. Yoshinaga, T. Sato, K. Asahi, B.P. Das, Probing exotic phenomena at the interface of nuclear and particle physics with the electric dipole moments of diamagnetic atoms: A unique window to hadronic and semi-leptonic CP violation, Eur. Phys. J. A 53 (3) (2017) 54, arXiv:1703.01570 [hep-ph].

[420] M. Gonzalez-Alonso, O. Naviliat-Cuncic, N. Severijns, New physics searches in nuclear and neutron $\beta$ decay, Prog. Part. Nucl. Phys. 104 (2019) 165-223, arXiv:1803.08732 [hep-ph].

[421] Z.-B. Kang, A. Prokudin, P. Sun, F. Yuan, Extraction of quark transversity distribution and collins fragmentation functions with QCD evolution, Phys. Rev. D 93 (1) (2016) 014009, arXiv:1505.05589 [hep-ph].

[422] M. Radici, A. Bacchetta, First extraction of transversity from a global analysis of electron-proton and proton-proton data, Phys. Rev. Lett. 120 (19) (2018) 192001, arXiv:1802.05212 [hep-ph].

[423] G.S. Bali, S. Collins, B. Glaessle, M. Gockeler, J. Najjar, R.H. Rodl, A. Schafer, R.W. Schiel, W. Söldner, A. Sternbeck, Nucleon isovector couplings from $N_{f}=2$ lattice QCD, Phys. Rev. D 91 (5) (2015) 054501, arXiv:1412.7336 [hep-lat].

[424] T. Bhattacharya, V. Cirigliano, R. Gupta, H.-W. Lin, B. Yoon, Neutron electric dipole moment and tensor charges from lattice QCD, Phys. Rev. Lett. 115 (21) (2015) 212002, arXiv:1506.04196 [hep-lat].

[425] C. Alexandrou, et al., Nucleon scalar and tensor charges using lattice QCD simulations at the physical value of the pion mass, Phys. Rev. D 95 (11) (2017) 114514, arXiv:1703.08788 [hep-lat]; Phys. Rev. D 96 (9) (2017) 099906, (erratum).

[426] A. Accardi, A. Bacchetta, Accessing the nucleon transverse structure in inclusive deep inelastic scattering, Phys. Lett. B 773 (2017) 632-638 arXiv:1706.02000 [hep-ph].

[427] A. Accardi, A. Signori, Quark fragmentation as a probe of dynamical mass generation, Phys. Lett. B 798 (2019) 134993, arXiv:1903.04458 [hep-ph].

[428] R. Gupta, B. Yoon, T. Bhattacharya, V. Cirigliano, Y.-C. Jang, H.-W. Lin, Flavor diagonal tensor charges of the nucleon from (2+1+1)-flavor lattice QCD, Phys. Rev. D 98 (9) (2018) 091501, arXiv:1808.07597 [hep-lat].

[429] STAR Collaboration, J. Adam, et al., Transverse spin transfer to $\Lambda$ and $\bar{\Lambda}$ hyperons in polarized proton-proton collisions at $\sqrt{s}=200 \mathrm{GeV}$, Phys. Rev. D 98 (9) (2018) 091103, arXiv:1808.08000 [hep-ex].

[430] N. Brambilla, et al., QCD and strongly coupled gauge theories: Challenges and perspectives, Eur. Phys. J. C 74 (10) (2014) 2981, arXiv:1404.3723 [hep-ph]. 
[431] STAR Collaboration, J. Adams, et al., Experimental and theoretical challenges in the search for the quark gluon plasma: The STAR collaboration's critical assessment of the evidence from RHIC collisions, Nuclear Phys. A 757 (2005) 102-183, arXiv:nucl-ex/0501009 [nucl-ex].

[432] BRAHMS Collaboration, I. Arsene, et al., Quark gluon plasma and color glass condensate at RHIC? The perspective from the BRAHMS experiment, Nuclear Phys. A 757 (2005) 1-27, arXiv:nucl-ex/0410020 [nucl-ex].

[433] STAR Collaboration, L. Adamczyk, et al., Centrality dependence of identified particle elliptic flow in relativistic heavy ion collisions at $\sqrt{S_{N N}}=7.7-62.4 \mathrm{GeV}$, Phys. Rev. C 93 (1) (2016) 014907, arXiv:1509.08397 [nucl-ex].

[434] STAR Collaboration, L. Adamczyk, et al., Elliptic flow of identified hadrons in Au+Au collisions at $\sqrt{s_{N N}}=7.7-62.4 \mathrm{GeV}$, Phys. Rev. C 88 (2013) 014902, arXiv:1301.2348 [nucl-ex].

[435] STAR Collaboration, L. Adamczyk, et al., Inclusive charged hadron elliptic flow in Au + Au collisions at $\sqrt{s_{N N}}=7.7-39$ GeV, Phys. Rev. C 86 (2012) 054908, arXiv:1206.5528 [nucl-ex].

[436] STAR Collaboration, L. Adamczyk, et al., Beam energy dependence of jet-quenching effects in Au+Au collisions at $\sqrt{S_{\mathrm{NN}}}=7.7,11.5,14.5,19.6$, 27, 39, and $62.4 \mathrm{GeV}$, Phys. Rev. Lett. 121 (3) (2018) 032301, arXiv:1707.01988 [nucl-ex].

[437] N. Brambilla, et al., Heavy quarkonium: progress, puzzles, and opportunities, Eur. Phys. J. C 71 (2011) 1534, arXiv:1010.5827 [hep-ph].

[438] CMS Collaboration, A.M. Sirunyan, et al., Suppression of excited $\Upsilon$ states relative to the ground state in $\mathrm{Pb}-\mathrm{Pb}$ collisions at $\sqrt{S_{\mathrm{NN}}}=5.02 \mathrm{TeV}$, Phys. Rev. Lett. 120 (14) (2018) 142301, arXiv:1706.05984 [hep-ex].

[439] CMS Collaboration, V. Khachatryan, et al., Suppression of $\Upsilon(1 S), \Upsilon(2 S)$ and $\Upsilon(3 S)$ production in PbPb collisions at $\sqrt{s_{\mathrm{NN}}}=2.76 \mathrm{TeV}$, Phys. Lett. B 770 (2017) 357-379, arXiv:1611.01510 [nucl-ex].

[440] CMS Collaboration, S. Chatrchyan, et al., Observation of sequential upsilon suppression in PbPb collisions, Phys. Rev. Lett. 109 (2012) 222301, arXiv:1208.2826 [nucl-ex]; Phys. Rev. Lett. 120 (19) (2018) 199903, (erratum).

[441] B. Trzeciak, C. Da Silva, E.G. Ferreiro, C. Hadjidakis, D. Kikola, J.P. Lansberg, L. Massacrier, J. Seixas, A. Uras, Z. Yang, Heavy-ion physics at a fixed-target experiment using the LHC proton and lead beams (AFTER@LHC): Feasibility studies for quarkonium and Drell-Yan production, Few Body Syst. 58 (5) (2017) 148, arXiv:1703.03726 [nucl-ex].

[442] E.G. Ferreiro, J.-P. Lansberg, Is bottomonium suppression in proton-nucleus and nucleus-nucleus collisions at LHC energies due to the same effects?, J. High Energy Phys. 10 (2018) 094, arXiv:1804.04474 [hep-ph]; J. High Energy Phys. 03 (2019) 063, (erratum);

[443] CMS Collaboration, S. Chatrchyan, et al., Indications of suppression of excited $\Upsilon$ states in PbPb collisions at $\sqrt{S_{N N}}=2.76$ TeV Phys. Rev. Lett. 107 (2011) 052302, arXiv:1105.4894 [nucl-ex].

[444] C. Mayer, Charmonium photoproduction in ultra-peripheral and peripheral Pb-Pb and p-Pb collisions with ALICE at the LHC. Quark Matter May 14, 2018

[445] STAR Collaboration, L. Adamczyk, et al., Energy dependence of $J / \psi$ production in Au+Au collisions at $\sqrt{S_{N N}}=39,62.4$ and $200 \mathrm{GeV}$ Phys Lett. B 771 (2017) 13-20, arXiv:1607.07517 [hep-ex].

[446] STAR Collaboration, L. Adamczyk, et al., Measurement of $D^{0}$ azimuthal anisotropy at midrapidity in Au+Au collisions at $\sqrt{S_{N N}}=200 \mathrm{GeV}$ Phys Rev. Lett. 118 (21) (2017) 212301, arXiv:1701.06060 [nucl-ex].

[447] STAR Collaboration, L. Adamczyk, et al., Observation of $D^{0}$ meson nuclear modifications in Au+Au collisions at $\sqrt{S_{N N}}=200 \mathrm{GeV}$ Phys. Rev. Lett. 113 (14) (2014) 142301, arXiv:1404.6185 [nucl-ex].

[448] STAR Collaboration, L. Adamczyk, et al., Elliptic flow of electrons from heavy-flavor hadron decays in Au + Au collisions at $\sqrt{s_{\mathrm{NN}}}=200,62.4$ and 39 GeV Phys. Rev. C 95 (3) (2017) 034907, arXiv:1405.6348 [hep-ex].

[449] PHENIX Collaboration, A. Adare, et al., Heavy quark production in $p+p$ and energy loss and flow of heavy quarks in Au+Au collisions at $\sqrt{s_{N N}}=200 \mathrm{GeV}$ Phys. Rev. C 84 (2011) 044905, arXiv:1005.1627 [nucl-ex].

[450] A. Beraudo, et al., Extraction of heavy-flavor transport coefficients in QCD matter Nuclear Phys. A 979 (2018) 21-86, arXiv:1803.03824 [nucl-th]

[451] ATLAS Collaboration, G. Aad, et al., Measurement of event-plane correlations in $\sqrt{s_{N N}}=2.76 \mathrm{TeV}$ lead-lead collisions with the ATLAS detector Phys. Rev. C 90 (2) (2014) 024905, arXiv:1403.0489 [hep-ex].

[452] ATLAS Collaboration, Correlated long-range mixed-harmonic fluctuations in $p p, p+\mathrm{Pb}$ and low-multiplicity Pb+Pb collisions with the ATLAS detector 2018, ATLAS-CONF-2018-012.

[453] ATLAS Collaboration, M. Aaboud, et al., Measurement of longitudinal flow decorrelations in $\mathrm{Pb}+\mathrm{Pb}$ collisions at $\sqrt{s_{\mathrm{NN}}}=2.76$ and 5.02 TeV with the ATLAS detector Eur. Phys. J. C 78 (2) (2018) 142, arXiv:1709.02301 [nucl-ex].

[454] B. Schenke, S. Jeon, C. Gale, Elliptic and triangular flow in event-by-event (3+1)D viscous hydrodynamics Phys. Rev. Lett. 106 (2011) 042301 arXiv: 1009.3244 [hep-ph].

[455] P. Bozek, W. Broniowski, J. Moreira, Torqued fireballs in relativistic heavy-ion collisions Phys. Rev. C 83 (2011) 034911, arXiv:1011.3354 [nucl-th].

[456] J. Jia, P. Huo, Forward-backward eccentricity and participant-plane angle fluctuations and their influences on longitudinal dynamics of collective flow Phys. Rev. C 90 (3) (2014) 034915, arXiv:1403.6077 [nucl-th].

[457] K. Xiao, F. Liu, F. Wang, Event-plane decorrelation over pseudorapidity and its effect on azimuthal anisotropy measurements in relativistic heavy-ion collisions Phys. Rev. C 87 (1) (2013) 011901, arXiv:1208.1195 [nucl-th].

[458] X.-Y. Wu, L.-G. Pang, G.-Y. Qin, X.-N. Wang, Longitudinal fluctuations and decorrelations of anisotropic flows at energies available at the CERN Large Hadron Collider and at the BNL Relativistic Heavy Ion Collider Phys. Rev. C 98 (2) (2018) 024913, arXiv:1805.03762 [nucl-th].

[459] S. Chatterjee, P. Bozek, Large directed flow of open charm mesons probes the three dimensional distribution of matter in heavy ion collisions Phys. Rev. Lett. 120 (19) (2018) 192301, arXiv:1712.01189 [nucl-th].

[460] D. Keane, The beam energy scan at the relativistic heavy ion collider J. Phys. Conf. Ser. 878 (1) (2017) 012015.

[461] J. Brewer, S. Mukherjee, K. Rajagopal, Y. Yin, Searching for the QCD critical point via the rapidity dependence of cumulants Phys. Rev. C 98 (6) (2018) 061901, arXiv:1804.10215 [hep-ph].

[462] CMS Collaboration, V. Khachatryan, et al., Observation of long-range near-side angular correlations in proton-proton collisions at the LHC J. High Energy Phys. 09 (2010) 091, arXiv:1009.4122 [hep-ex].

[463] CMS Collaboration, C.M.S. Collaboration, S. Chatrchyan, et al., Observation of long-range near-side angular correlations in proton-lead collisions at the LHC Phys. Lett. B 718 (2013) 795-814, arXiv:1210.5482 [nucl-ex].

[464] PHENIX Collaboration, A. Adare, et al., Quadrupole anisotropy in dihadron azimuthal correlations in central $d+$ Au collisions at $\sqrt{S_{N N}}=200$ GeV Phys. Rev. Lett. 111 (21) (2013) 212301, arXiv:1303.1794 [nucl-ex].

[465] CMS Collaboration, S. Chatrchyan, et al., Multiplicity and transverse momentum dependence of two- and four-particle correlations in pPb and PbPb collisions Phys. Lett. B 724 (2013) 213-240, arXiv:1305.0609 [nucl-ex].

[466] PHENIX Collaboration, C. Aidala, et al., Measurements of multiparticle correlations in $d+$ Au collisions at 200, 62.4, 39, and 19.6 GeV and $p+$ Au collisions at $200 \mathrm{GeV}$ and implications for collective behavior Phys. Rev. Lett. 120 (6) (2018) 062302, arXiv:1707.06108 [nucl-ex].

[467] CMS Collaboration, C. Collaboration, Observation of prompt $\mathrm{J} / \psi$ meson elliptic flow in high-multiplicity pPb collisions at $\sqrt{S_{\mathrm{NN}}}=8.16 \mathrm{TeV}$, CMS-PAS-HIN-18-010.

[468] CMS Collaboration, A.M. Sirunyan, et al., Elliptic flow of charm and strange hadrons in high-multiplicity pPb collisions at $\sqrt{s_{\mathrm{NN}}}=8.16$ TeV Phys. Rev. Lett. 121 (8) (2018) 082301, arXiv:1804.09767 [hep-ex]. 
[469] ALICE Collaboration, J. Adam, et al., Enhanced production of multi-strange hadrons in high-multiplicity proton-proton collisions Nat. Phys. 13 (2017) 535-539, arXiv: 1606.07424 [nucl-ex].

[470] STAR Collaboration, J. Adam, et al., $J / \psi$ Production cross section and its dependence on charged-particle multiplicity in $p+p$ collisions at $\sqrt{s}$ = $200 \mathrm{GeV}$ Phys. Lett. B 786 (2018) 87-93, arXiv:1805.03745 [hep-ex].

[471] ALICE Collaboration, B. Abelev, et al., $J / \psi$ Production as a function of charged particle multiplicity in $p p$ collisions at $\sqrt{s}=7$ TeV Phys. Lett. B 712 (2012) 165-175, arXiv:1202.2816 [hep-ex].

[472] ALICE Collaboration, J. Adam, et al., Measurement of charm and beauty production at central rapidity versus charged-particle multiplicity in proton-proton collisions at $\sqrt{s}=7 \mathrm{TeV}$ J. High Energy Phys. 09 (2015) 148, arXiv:1505.00664 [nucl-ex].

[473] E.G. Ferreiro, C. Pajares, High multiplicity pp events and $J / \psi$ production at LHC Phys. Rev. C 86 (2012) 034903, arXiv:1203.5936 [hep-ph].

[474] K. Werner, B. Guiot, I. Karpenko, T. Pierog, Analysing radial flow features in $\mathrm{p}-\mathrm{Pb}$ and p-p collisions at several TeV by studying identified particle production in EPOS3 Phys. Rev. C 89 (6) (2014) 064903, arXiv:1312.1233 [nucl-th].

[475] L. Yan, J.-Y. Ollitrault, Universal fluctuation-driven eccentricities in proton-proton, proton-nucleus and nucleus-nucleus collisions Phys. Rev. Lett. 112 (2014) 082301, arXiv:1312.6555 [nucl-th].

[476] P. Bozek, W. Broniowski, Collective dynamics in high-energy proton-nucleus collisions Phys. Rev. C 88 (1) (2013) 014903, arXiv:1304.3044 [nucl-th].

[477] R.D. Weller, P. Romatschke, One fluid to rule them all: viscous hydrodynamic description of event-by-event central $\mathrm{p}+\mathrm{p}, \mathrm{p}+\mathrm{Pb}$ and $\mathrm{Pb}+\mathrm{Pb}$ collisions at $\sqrt{s}=5.02$ TeV Phys. Lett. B 774 (2017) 351-356, arXiv:1701.07145 [nucl-th].

[478] H. Mntysaari, B. Schenke, C. Shen, P. Tribedy, Imprints of fluctuating proton shapes on flow in proton-lead collisions at the LHC Phys. Lett. B 772 (2017) 681-686, arXiv:1705.03177 [nucl-th].

[479] L. He, T. Edmonds, Z.-W. Lin, F. Liu, D. Molnar, F. Wang, Anisotropic parton escape is the dominant source of azimuthal anisotropy in transport models Phys. Lett. B 753 (2016) 506-510, arXiv:1502.05572 [nucl-th].

[480] K. Dusling, R. Venugopalan, Azimuthal collimation of long range rapidity correlations by strong color fields in high multiplicity hadron-hadron collisions Phys. Rev. Lett. 108 (2012) 262001, arXiv:1201.2658 [hep-ph].

[481] J.R. Christiansen, P.Z. Skands, String formation beyond leading colour J. High Energy Phys. 08 (2015) 003, arXiv:1505.01681 [hep-ph].

[482] Z. Citron, et al., Report from working group 5 2019, arXiv:1812.06772 [hep-ph].

[483] CMS Collaboration, C. Collaboration, Studies of charm and beauty long-range correlations in pp and pPb collisions.

[484] CMS Collaboration, S. Chatrchyan, et al., Measurement of isolated photon production in $p p$ and PbPb collisions at $\sqrt{S_{N N}}=2.76$ TeV Phys. Lett. B 710 (2012) 256-277, arXiv:1201.3093 [nucl-ex].

[485] CMS Collaboration, S. Chatrchyan, et al., Study of $W$ boson production in PbPb and pp collisions at $\sqrt{S_{N N}}=2.76 \mathrm{TeV}$ Phys. Lett. B 715 (2012) 66-87, arXiv:1205.6334 [nucl-ex].

[486] CMS Collaboration, S. Chatrchyan, et al., Study of $\mathrm{Z}$ production in $\mathrm{PbPb}$ and pp collisions at $\sqrt{S_{\mathrm{NN}}}=2.76 \mathrm{TeV}$ in the dimuon and dielectron decay channels J. High Energy Phys. 03 (2015) 022, arXiv:1410.4825 [nucl-ex].

[487] B.Z. Kopeliovich, H.J. Pirner, I.K. Potashnikova, I. Schmidt, Mutual boosting of the saturation scales in colliding nuclei Phys. Lett. B 697 (2011) 333-338, arXiv: 1007.1913 [hep-ph].

[488] W. Scandale, Optical layout for the measurement of Short Living Baryon Magnetic Moment using Bended Crystals at LHC. Physics Beyond Collider, Kickoff Workshop, September 6-7, 2016. 